(b)

( 151

$\left.x^{-1}+9\right)^{2}$

- is

$f^{2}+2+2+2$

Liquefaction of

1

ing

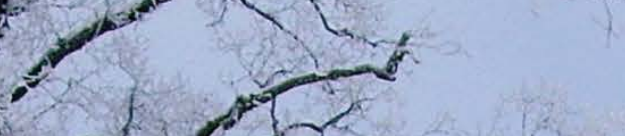

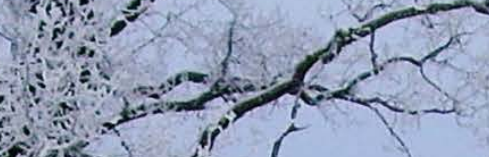

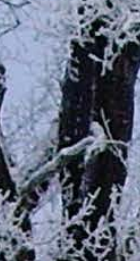

\% 8

2.

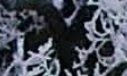

is:

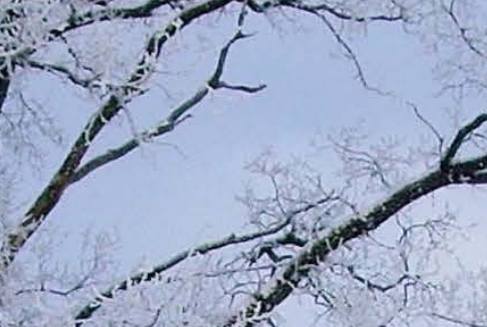

(x) $x$,<smiles>C1CCCC1</smiles><smiles>C1[Te][Te]1</smiles>
yignocellulose

sar 4

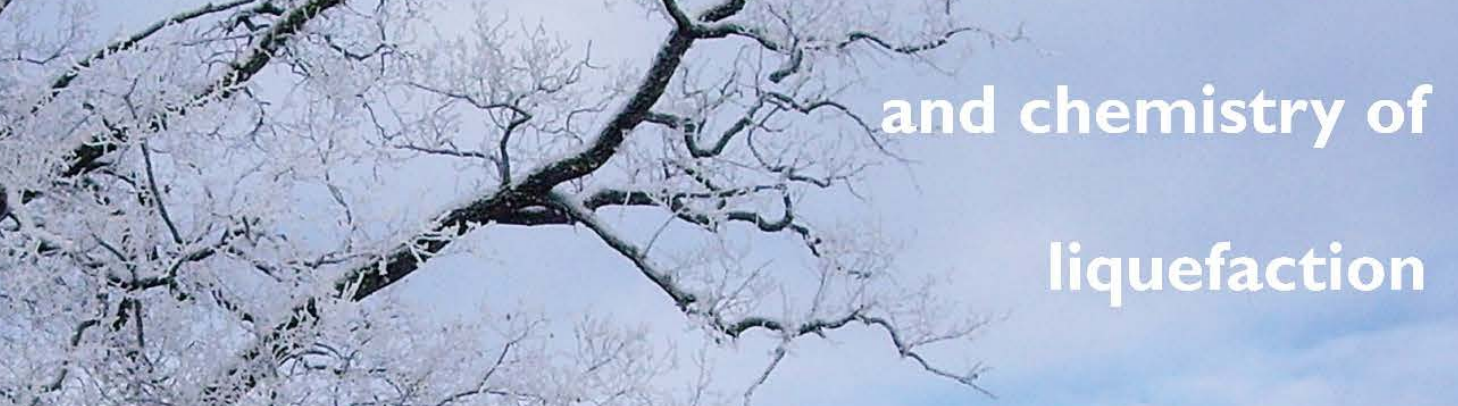

(1)

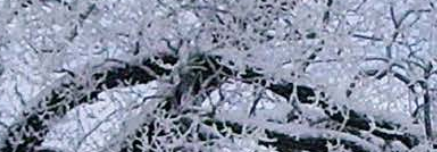

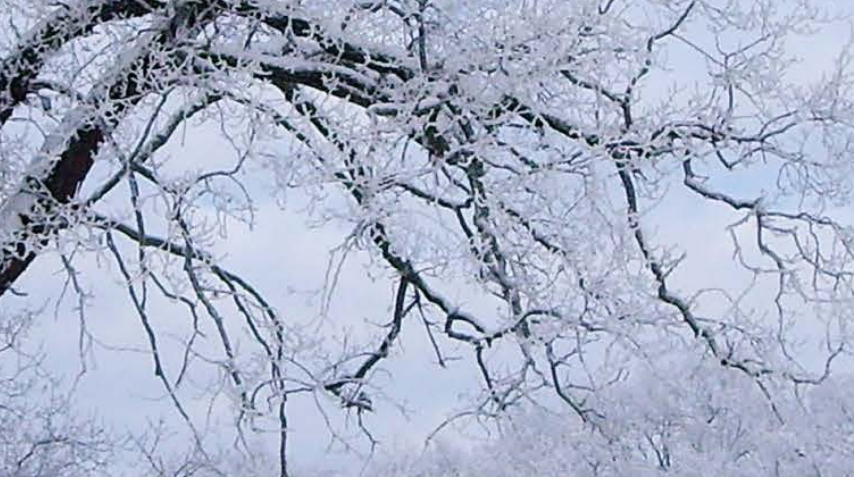

I.

$+6$

站

75

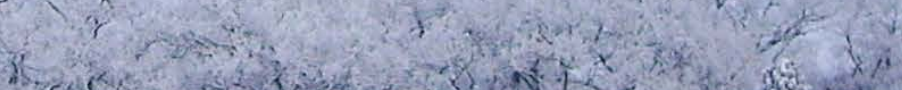

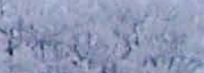

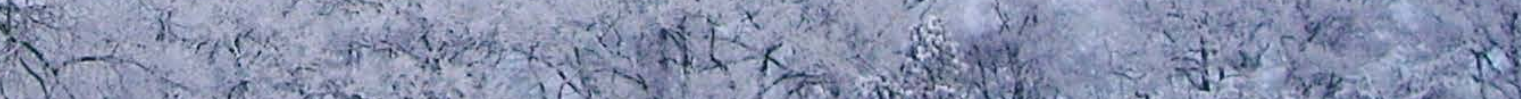

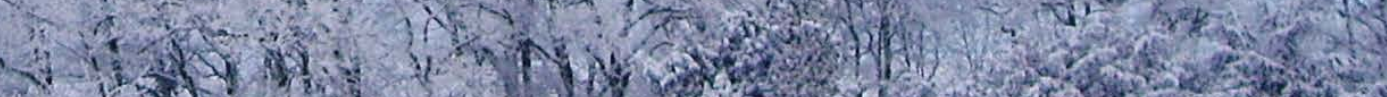

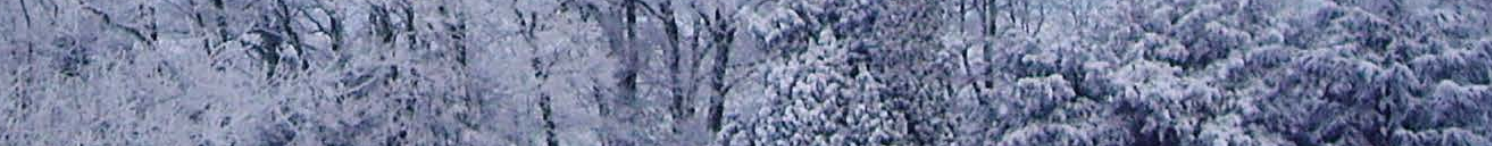

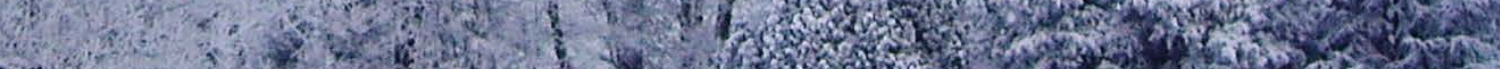

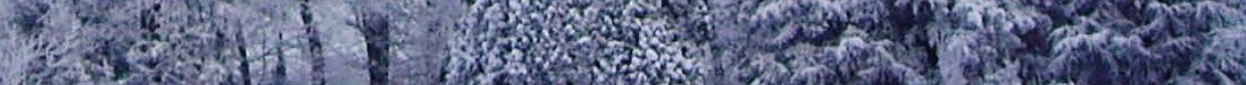
1 Maria castelvi Bamés 4

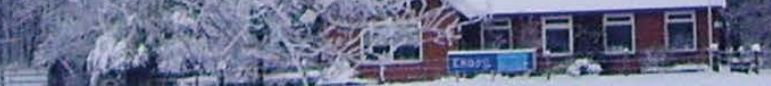




\section{LIQUEFACTION OF LIGNOCELLULOSE}

BIO-CRUDE, CHAR AND CHEMISTRY OF LIQUEFACTION 


\section{Promotion committee:}

$\begin{array}{lll}\text { Chairman: } & \text { Prof. dr. ir. J.W.M. Hilgenkamp } & \text { University of Twente } \\ \text { Promotors: } & \text { Prof. dr. S.R.A. Kersten } & \text { University of Twente } \\ & \text { Prof. dr. J.P. Lange } & \text { University of Twente } \\ \text { Assistant promotor: } & \text { Dr. ir. G. van Rossum } & \text { Shell, The Netherlands } \\ \text { Members: } & \text { Prof. dr. ir. L. Lefferts } & \text { University of Twente } \\ & \text { Prof. dr. ir. J. Huskens } & \text { University of Twente } \\ & \text { Prof. dr. ir. W. Prins } & \text { University of Gent } \\ & \text { prof. dr. ir. B.M. Weckhuysen } & \text { University of Utrecht }\end{array}$

The research described in this thesis was financially supported by Shell Global Solutions, Amsterdam, The Netherlands.

Liquefaction of lignocellulose: Bio-crude, char and chemistry of liquefaction

Cover design: Maria Castellví Barnés

ISBN: 978-90-365-4133-6

DOI-number: 10.3990/1.9789036541336

URL: http://dx.doi.org/10.3990/1.9789036541336

Printed by Gildeprint - Enschede, The Netherlands

(C) 2016 Maria Castellví Barnés, Enschede, The Netherlands 


\title{
LIQUEFACTION OF LIGNOCELLULOSE BIO-CRUDE, CHAR AND CHEMISTRY OF LIQUEFACTION
}

\author{
DISSERTATION
}

to obtain

the degree of doctor at the University of Twente,

on the authority of the rector magnificus,

Prof.dr. H. Brinksma,

on account of the decision of the graduation committee,

to be publicly defended

on Friday $27^{\text {th }}$ of May, 2016 at 12:45

by

\section{Maria Castellví Barnés}

born on $17^{\text {th }}$ of March, 1988

in Barcelona, Spain 
This dissertation has been approved by:

Prof. dr. S.R.A. Kersten (Promotor)

Prof. dr. J.P. Lange (Promotor)

Dr. ir. G. van Rossum (Assistant Promotor) 
To my family

Per estar sempre al meu costat 



\section{Contents}

I Chapter I

Introduction

I5 Chapter 2

Reagents and experimental procedure

39 Chapter 3

Solvent, process parameter and recycle oil screening

\section{Chapter 4}

Bio-crude characterization based on GPC preparative fractionation

83 Chapter 5

Chemistry of the Liquefaction of wood and its model components

\section{Chapter 6}

Effect of the reaction medium during liquefaction of lignocellulose

\section{Chapter 7}

Summary, conclusions and recommendations

\section{Samenvatting}

\section{Resum}

\section{List of symbols}

\section{List of publications}

\section{I Supporting information}

\section{Acknowledgements}





\section{Chapter I}

\section{Introduction}




\section{Importance of bio-fuels}

The discovery of fossil fuels at the beginning of the industrial revolution, allowed a huge development of the human society [1,2]. This development has favoured an increase in global population and quality of life, but has also caused major changes in the earth environment, such as an increase in greenhouse emissions (contributing to climate change), a decrease in bio-diversity and an imbalance of the phosphorous and nitrogen cycles (used as fertilizers) [1-4]. In the coming years, the growth in global population and prosperity, especially of developing countries (e.g. China or India), is expected to increase even further the world's energy demand $[1,5,6]$. However, more sustainable energy sources are needed and, therefore, alternatives to fossil energy sources are being explored. Furthermore, the need to secure energy supply (currently controlled by a limited number of countries) and the economic development they would provide (especially in the rural areas) are strong motivations for governments around the world to support the development of these alternatives to conventional energy sources [1, 6-8].

Nowadays, a quarter of the world's energy consumption is used for transportation $[9,10]$. This sector is presently powered by liquid fuels because of their high energy densities and because they are easy to store and transport. Here, bio-fuels stand as a sustainable substitute for fossil fuels, as they are carbon-based and, therefore, fit in the carbon-based (fossil) fuel existing infrastructure. Furthermore, unlike other energies like solar or wind, bio-fuels can provide a continuous energy supply and have less dependence on geographical location [1, 6-8].

In 2010, bio-fuels (mainly ethanol and bio-diesel) provided less than $2.5 \%$ of the total energy consumption used in the transport sector [7, 10]. However, according to the International Energy Agency (IEA), $26 \%$ of the total transport fuel demand in 2050 could be provided by bio-fuels, with $90 \%$ of those being second generation biofuels [11]. 


\section{From biomass to bio-fuel}

\section{I. Lignocellulosic biomass}

Biomass is considered a carbon-neutral renewable energy source. However, economic, social and environmental impacts have to be taken into account when selecting the appropriate biomass to produce bio-fuels.

The first generation of bio-fuels was obtained from sugars, starches and vegetable and animal fats. However, those biomass sources compete with the food industry. To overcome this problem, second generation bio-fuels were developed. These fuels are obtained from lignocellulosic biomass, which can be obtained in large amounts, for example, from agricultural and forestry residues. However, to guarantee a low environmental impact, the production of lignocellulose should take into account the type of land used for its cultivation (co-production with food crops or use of degraded or abandoned lands instead of replacing forests or other plantations), the nutrient balances (e.g. nitrogen and phosphate) or the water sources [7, 8, 12-14].

Lignocellulose is the fibrous material forming the essential part of the cell wall of plants. It is mainly composed of three types of polymer, namely cellulose (40-50 $\mathrm{wt} \%)$, hemicellulose (15-35 $\mathrm{wt} \%$ ) and lignin (15-35 wt\%), which are connected to each other, providing strength and resistance to the material. Other components such as starch, proteins, fats or mineral salts are also found in smaller amounts [1519].

Cellulose is a carbohydrate composed of glucose units arranged in linear chains. These linear chains are connected to each other by hydrogen bonds and organized in crystalline and amorphous regions. Generally, wood cellulose has a polymerization degree of 10000 and a molecular weight of $1 \mathrm{MDa}$. 
Hemicellulose is an amorphous carbohydrate composed of various sugar monomers (i.e. glucose, mannose and galactose) arranged into branched polymers. It has a polymerization degree of 200 and a molecular weight of approximately $30 \mathrm{kDa}$.

Lignin is an aromatic polymer composed of propyl-phenol units attached to each other by ether or carbon-carbon linkages. It has a molecular weight up to $20 \mathrm{kDa}$, corresponding to a polymerization degree of approximately 120 .

\subsection{Liquefaction}

Compared to traditional (fossil) fuels, biomass has high oxygen and water content that decreases its combustion value. It has also a very low density, which is detrimental for its transportation and storage $[7,20]$. Biomass is thus converted into a liquid product that has higher energy density and is easier to handle and store.

Numerous techniques can be used to convert biomass into a liquid product, involving either thermochemical (liquefaction, pyrolysis, and gasification), chemical (hydrolysis) or biological (fermentation) routes. Occasionally, biomass conversion is preceded by a pre-treatment to fractionate the biomass (e.g. sugar isolation before fermentation) or to debilitate and make the lignocellulose structure more accessible (e.g. mild hydrolysis or uncatalysed steam explosion) [6, 7, 19, 21].

In the current work we used liquefaction for the conversion of biomass into biocrude, as it presents several advantages over other thermochemical processes. For instance, liquefaction is generally performed at milder temperatures than pyrolysis or gasification [7, 19], and it leads to higher oil yields than fast pyrolysis (oil yield > $90 \mathrm{C} \%$ vs 50-55 C\%) [22-28]. In addition, liquefaction can be performed with wet biomass. In fact, liquefaction is frequently performed in water (hydrothermal liquefaction) or in solvent mixtures containing water.

Research on biomass liquefaction started in the early 1970s at the Pittsburg Energy Research Centre (PERC). After those first studies, a lot of liquefaction processes have 
been developed using a wide range of conditions (e.g. temperature, liquefaction medium, sub- or super-critical conditions, use of catalyst or use of reactive gases). For more extensive information about liquefaction studies the reader can consult several publications reviewing the topic [17, 22, 29-32]. Despite all the research performed on liquefaction, the process has not yet reached a commercial state. The reasons are that liquefaction processes are generally performed under extreme process conditions and tend to present problems regarding high bio-crude viscosities, formation of solids or loss of light species in aqueous phases. Furthermore, the produced bio-crude still requires upgrading if it is to be used as high quality fuel.

Thus, further research is required to develop a technologically and economically feasible liquefaction process. However, studies should not only focus on process optimization, but also on the understanding of the process and the effect that the liquefaction conditions have on the product distribution and the oil composition and quality.

Our study focuses on a direct liquefaction process that does not require biomass pretreatment and that yields a bio-crude that can be blended with fossil fuels and introduced into existing refinery units for its further upgrading. To have an economically feasible process, certain requirements were defined. Liquefaction must be operated at relatively mild conditions, namely liquefaction temperatures around $300^{\circ} \mathrm{C}$ and pressures lower than 60 bar. To maintain low pressures, use of reactive gases or liquefaction media with low boiling points must be avoided. The liquefaction medium should consist of a cheap solvent that does not need to be separated from the final product. This can include cheap refinery streams, or a fraction of the produced bio-crude. Finally, the use of catalysts should also be avoided to prevent contamination of the resulting bio-crude and maintain low process costs. 


\subsection{Bio-crude}

Despite presenting better fuel properties than the initial biomass, bio-crude still contains more oxygen and has higher molecular weight $(\mathrm{Mw})$ than conventional fuels (Figure 1). This leads to lower energy content, low volatility, high viscosities, coking tendency, chemical instability (they tend to condense and polymerize with time) and corrosiveness. Furthermore, bio-crude has low solubility in hydrocarbons (important if it needs to be blended with fossil fuels) and is incompatible with some of the materials used in the existing fuel infrastructure [15, 19, 33]. Therefore, upgrading of the bio-crude is essential to obtain a high quality fuel. The most common techniques for bio-crude upgrading are hydrodeoxygenation and zeolite upgrading (catalytic cracking). Hydrodeoxygenation produces aliphatic and aromatic hydrocarbons via hydrogen addition and oxygen removal. Zeolite upgrading or catalytic cracking produces aromatics, light alkanes and coke via a number of reactions including dehydration, cracking, polymerization, deoxygenation, and aromatization [19, 33-35].

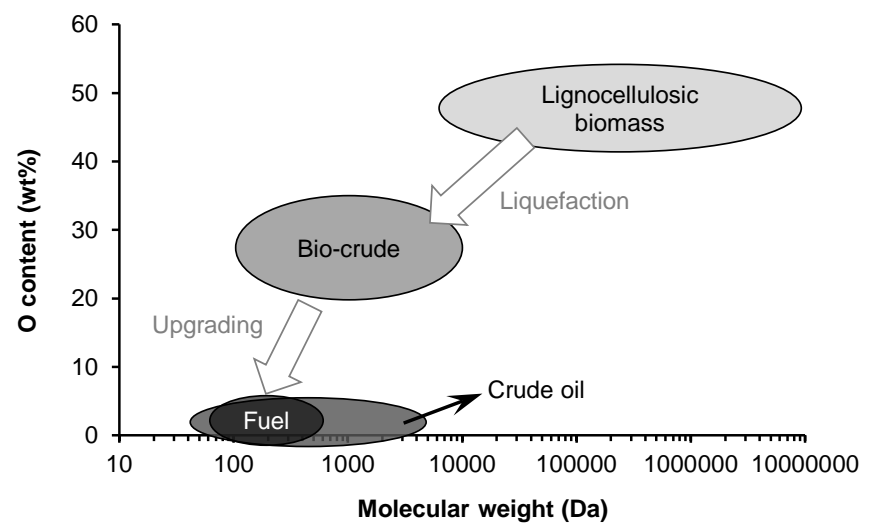

Figure 1. Oxygen content and Mw of initial biomass, bio-crude and bio-fuel [39].

A good characterization of the bio-crude is essential to determine its quality and decide which treatment is required for its upgrading. Many techniques are used for 
bio-crude characterization, such as gas chromatography (GC), two-dimensional GC (GCxGC), liquid chromatography (LC, including GPC), nuclear magnetic resonance (NMR), Fourier transformed infrared spectroscopy (FTIR) or high resolution mass spectrometry (HR-MS). Sometimes, sample preparation or pre-treatment (e.g. fractionation, extraction or distillation) is required to obtain more detailed information [36, 37]. However, some of these techniques suffer from practical limitations. For instance, gas chromatography can only detect the light components of the bio-crude [36, 38], or NMR and FTIR analyses may be obstructed if the biocrude is highly diluted in the liquefaction solvent.

\section{Scope and outline of the thesis}

The work presented in this thesis focuses on the characterization of the liquefaction products, the chemistry of the liquefaction process in terms of reactivity of biomass components, and the role of the liquefaction medium. This study was performed in parallel to the work of S. Kumar [29], which focused on the engineering and process design of the liquefaction.

In chapter 2, all reagents and materials, experimental procedures, characterization techniques and the product definitions are presented.

In chapter 3, we present a fast screening of the effect of various process parameters (i.e. liquefaction solvent, temperature and time) on product distribution and oil quality. Experiments with recycling of the produced bio-crude as liquefaction medium are also shown. The results obtained in this chapter are used to define the research questions that will be addressed in the following chapters.

The excess of liquefaction solvent in the final liquid product (>90 $\mathrm{wt} \%$ ) prevented a proper characterization of the liquefaction bio-crude. Due the high boiling point of the liquefaction solvent and its similar chemical composition with the bio-crude, conventional fractionation techniques (e.g. distillation or solvent extraction) were not suitable for bio-crude isolation. In chapter 4 we develop a characterization 
procedure based on preparative GPC fractionation to isolate the bio-crude from the liquefaction solvent. Furthermore, the heavy and light fractions of the bio-crude could be separated and analysed independently. Two liquefaction bio-crudes were studied and compared to fast pyrolysis oil.

In chapter 3 we observed that formation of heavy species in the bio-crude is a major drawback of the liquefaction process. Furthermore, char formation should be minimized to increase the efficiency of the process. Chapter 5 focuses on the liquefaction of wood and its main components (namely cellulose, hemicellulose and lignin). The aim of this chapter is to reveal the reaction mechanisms occurring during lignocellulose liquefaction and to determine the origin of the undesired products (heavy species in the bio-crude, char and gas).

In chapter 3 we observed that different liquefaction media lead to different product distribution. However, the role of the medium during liquefaction is not yet fully understood. Chapter 6 studies the effect that the liquefaction medium has on the product distribution. Based on the obtained results, we were able to define how the optimum liquefaction medium that would maximize oil yield and prevent char formation would be.

Chapter 7 summarizes all the work presented in this thesis and draws overall conclusions linking all the results presented in the various chapters. 


\section{References}

1. Groeneveld, M.J., The change from fossil to solar and biofuels needs our energy. Inaugural lecture., in TCCB. 2008, university of Twente: Enschede.

2. Steffen, W., Crutzen, P.J., and McNeill, J.R., The Anthropocene: Are Humans Now Overwhelming the Great Forces of Nature. AMBIO: A Journal of the Human Environment, 2007. 36(8): p. 614-621.

3. Steffen, W., Richardson, K., Rockström, J., Cornell, S.E., Fetzer, I., Bennett, E.M., Biggs, R., Carpenter, S.R., de Vries, W., de Wit, C.A., Folke, C., Gerten, D., Heinke, J., Mace, G.M., Persson, L.M., Ramanathan, V., Reyers, B., and Sörlin, S., Planetary boundaries: Guiding human development on a changing planet. Science, 2015. 347(6223).

4. Hofmann, D.J., Butler, J.H., and Tans, P.P., A new look at atmospheric carbon dioxide. Atmospheric Environment, 2009. 43(12): p. 2084-2086.

5. Shell International BV, Shell Energy Scenarios to 2050: Signals E Signposts. 2011.

6. Lange, J.P., Lignocellulose conversion: An introduction to chemistry, process and economics. Biofuels, Bioproducts and Biorefining, 2007. 1(1): p. 39-48.

7. Swaaij, W.v., Kersten, S., and Palz, W., Biomass power for the world. Pan Stanford Series on Renewable Energy, ed. W. Palz. Vol. 6. 2015, Singapore: Pan Stanford Publishing.

8. de Jong, W. and van Ommen, J.R., Introduction: socioeconomic aspects of biomass conversion, in Biomass as a Sustainable Energy Source for the Future. 2014, John Wiley \& Sons, Inc. p. 135.

9. US Energy Information Administration; Total energy; 2016. http://www.eia.gov/totalenergy/.

10. International Energy Agency; Statistics; 2016. http://www.iea.org/statistics/.

11. International Energy Agency, Sustainable production of second-generation biofuels. 2010.

12. Anwar, Z., Gulfraz, M., and Irshad, M., Agro-industrial lignocellulosic biomass a key to unlock the future bio-energy: A brief review. Journal of Radiation Research and Applied Sciences, 2014. 7(2): p. 163-173.

13. Biomass as a Sustainable Energy Source for the Future. 2014, New Jersey: John Wiley \& Sons, Inc. 
14. Lange, J.-P., Lewandowski, I., and Ayoub, P.M., Cellulosic Biofuels: A Sustainable Option for Transportation, in Sustainable Development in the Process Industries. 2010, John Wiley \& Sons, Inc. p. 171-198.

15. Lange, J.-P., Lignocellulose Conversion: An Introduction to Chemistry, Process and Economics, in Catalysis for Renewables. 2007, Wiley-VCH Verlag GmbH \& Co. KGaA. p. 21-51.

16. Rowell, R.M., Handbook of wood chemistry and wood composites. 2005, United States of America: CRC press.

17. Bouvier, J.M., Gelus, M., and Maugendre, S., Wood liquefaction-An overview. Applied Energy, 1988. 30(2): p. 85-98.

18. Horvath, A.L., Solubility of Structurally Complicated Materials: I. Wood. Journal of Physical and Chemical Reference Data, 2006. 35(1): p. 77-92.

19. Huber, G.W., Iborra, S., and Corma, A., Synthesis of Transportation Fuels from Biomass: Chemistry, Catalysts, and Engineering. Chemical Reviews, 2006. 106(9): p. 4044-4098.

20. Demirbaş, A., Biomass resource facilities and biomass conversion processing for fuels and chemicals. Energy Conversion and Management, 2001. 42(11): p. 1357-1378.

21. Fatih Demirbas, M., Biorefineries for biofuel upgrading: A critical review. Applied Energy, 2009. 86, Supplement 1(0): p. S151-S161.

22. Akhtar, J. and Amin, N.A.S., A review on process conditions for optimum bio-oil yield in hydrothermal liquefaction of biomass. Renewable and Sustainable Energy Reviews, 2011. 15(3): p. 1615-1624.

23. van Rossum, G., Zhao, W., Castellvi Barnes, M., Lange, J.-P., and Kersten, S.R.A., Liquefaction of Lignocellulosic Biomass: Solvent, Process Parameter, and Recycle Oil Screening. ChemSusChem, 2013. 7(1): p. 253 - 259.

24. Kumar, S., Lange, J.-P., Van Rossum, G., and Kersten, S.R.A., Liquefaction of Lignocellulose: Process Parameter Study To Minimize Heavy Ends. Industrial \& Engineering Chemistry Research, 2014. 53(29): p. 11668-11676.

25. Bridgwater, A.V., Review of fast pyrolysis of biomass and product upgrading. Biomass and Bioenergy, 2012. 38: p. 68-94.

26. Mohan, D., Pittman, C.U., and Steele, P.H., Pyrolysis of Wood/Biomass for Bio-oil: A Critical Review. Energy \& Fuels, 2006. 20(3): p. 848-889. 
27. Westerhof, R.J.M., Nygård, H.S., Van Swaaij, W.P.M., Kersten, S.R.A., and Brilman, D.W.F., Effect of particle geometry and microstructure on fast pyrolysis of beech wood. Energy and Fuels, 2012. 26(4): p. 2274-2280.

28. Piskorz, J., Radlein, D.S.A.G., Scott, D.S., and Czernik, S., Pretreatment of wood and cellulose for production of sugars by fast pyrolysis. Journal of Analytical and Applied Pyrolysis, 1989. 16(2): p. 127-142.

29. Kumar, S., Direct Liquefaction of Lignocellulose: Exploration, Design and Evaluation of Conceptual Processes, in Sustainable Process Technology. 2015, University of Twente: Netherlands. p. 166.

30. Moffatt, J.M. and Overend, R.P., Direct liquefaction of wood through solvolysis and catalytic hydrodeoxygenation: an engineering assessment. Biomass, 1985. 7(2): p. 99-123.

31. Toor, S.S., Rosendahl, L., and Rudolf, A., Hydrothermal liquefaction of biomass: A review of subcritical water technologies. Energy, 2011. 36(5): p. 2328-2342.

32. Huang, H.-j. and Yuan, X.-Z., Recent progress in the direct liquefaction of typical biomass. Progress in Energy and Combustion Science, 2015. 49: p. 59-80.

33. Xiu, S. and Shahbazi, A., Bio-oil production and upgrading research: A review. Renewable and Sustainable Energy Reviews, 2012. 16(7): p. 4406-4414.

34. Elliott, D.C., Transportation fuels from biomass via fast pyrolysis and hydroprocessing. Wiley Interdisciplinary Reviews: Energy and Environment, 2013. 2(5): p. 525-533.

35. Bridgwater, A.V., Catalysis in thermal biomass conversion. Applied Catalysis A: General, 1994. 116(1-2): p. 5-47.

36. Staš, M., Kubička, D., Chudoba, J., and Pospíšil, M., Overview of analytical methods used for chemical characterization of pyrolysis bio-oil. Energy and Fuels, 2014. 28(1): p. 385-402.

37. Kanaujia, P.K., Sharma, Y.K., Garg, M.O., Tripathi, D., and Singh, R., Review of analytical strategies in the production and upgrading of bio-oils derived from lignocellulosic biomass. Journal of Analytical and Applied Pyrolysis, 2014. 105(0): p. 55-74.

38. Branca, C., Giudicianni, P., and Di Blasi, C., GC/MS Characterization of Liquids Generated from Low-Temperature Pyrolysis of Wood. Industrial \& Engineering Chemistry Research, 2003. 42(14): p. 3190-3202.

39. Front Matter, in Catalysis for Renewables. 2007, Wiley-VCH Verlag GmbH \& Co. KGaA. p. I-XXIII. 


$$
\frac{\bar{A}}{\bar{O}}
$$




\section{Chapter 2}

Reagents and experimental procedure 
This chapter contains all the information concerning the materials, experimental setups, procedures, and analytical equipment used to perform all the experiments and analyses presented in this thesis. Definitions and calculation methods are also introduced. 


\section{Materials and reagents}

Pine wood was purchased from Rettenmaier \& Söhne GmbH (Germany), grinded and sieved to a particle size below $0.5 \mathrm{~mm}$ and, finally, dried at $105^{\circ} \mathrm{C}$ for 24 hours. Organosolv lignin was prepared from the pine wood described above following the method described by Huijgen et al [1]. Approximately 300 grams of wood were treated at $200^{\circ} \mathrm{C}$ during 60 minutes in $2.5 \mathrm{~kg}$ of an ethanol:water mixture $(60: 40 \mathrm{wt} \%)$ in a $5 \mathrm{~L}$ autoclave. The liquid product was slowly added onto cold water to precipitate the lignin, which was then filtered, washed and dried. Composition and characterization of the wood and the organosolv lignin are discussed in the following section (1.1).

The chemicals and reagents used for the characterization and the experiments are shown in Table 1 and Table 2.

Table 1. Chemicals used in the characterization.

\begin{tabular}{|c|c|c|c|c|}
\hline Chemical & Formula & Distributor & Purity & Comments \\
\hline Tetrahydrofuran (THF) & $\mathrm{C}_{4} \mathrm{H}_{8} \mathrm{O}$ & Sigma Aldrich & $99.9 \%$ & $\begin{array}{l}\text { Used for GPC measurements } \\
\text { Used for GPC fractionation }\end{array}$ \\
\hline $\begin{array}{l}\text { Deuterated methyl } \\
\text { sulfoxide (DMSO) }\end{array}$ & $\mathrm{C}_{2} \mathrm{D}_{6} \mathrm{OS}$ & $\begin{array}{l}\text { Acros } \\
\text { Organics }\end{array}$ & $99.8 \%$ & $\begin{array}{l}\text { Used for }{ }^{13} \mathrm{C}-\mathrm{NMR} \text { measurements } \\
\text { Contained } 0.03 \% \text { of trimethyl silane }\end{array}$ \\
\hline $\begin{array}{l}\text { Chromium (III) } \\
\text { acetylacetonate }\end{array}$ & $\mathrm{Cr}(\mathrm{AcAc})$ & Sigma Aldrich & $99.99 \%$ & Used for ${ }^{13} \mathrm{C}-\mathrm{NMR}$ measurements \\
\hline Deuterated acetone & $\mathrm{C}_{3} \mathrm{D}_{6} \mathrm{O}$ & VWR & $99.8 \%$ & Used for ${ }^{1} \mathrm{H}-\mathrm{NMR}$ measurements \\
\hline Acetone & $\mathrm{C}_{3} \mathrm{H}_{6} \mathrm{O}$ & Sigma Aldrich & $>99.5 \%$ & Used for GC-MS measurements \\
\hline Acetone & $\mathrm{C}_{3} \mathrm{H}_{6} \mathrm{O}$ & $\begin{array}{l}\text { Atlas Assink } \\
\text { Chemie }\end{array}$ & $>99.5 \%$ & $\begin{array}{l}\text { Used for product recovery and } \\
\text { washing of solid residue }\end{array}$ \\
\hline Hydranal Composite 5 & - & Sigma Aldrich & - & Used for KFT measurements \\
\hline $\begin{array}{l}\text { Methanol:dichloromethane } \\
\text { (volume ratio of } 3: 1 \text { ) }\end{array}$ & $\begin{array}{l}\mathrm{CH}_{4} \mathrm{O} \\
\mathrm{CH}_{2} \mathrm{Cl}_{2}\end{array}$ & $\begin{array}{l}\text { Sigma Aldrich } \\
\text { Sigma Aldrich }\end{array}$ & $\begin{array}{l}\geq 99.9 \% \\
\geq 99.8 \%\end{array}$ & Used for KFT measurements \\
\hline
\end{tabular}


Table 2. Chemicals used in the experiments.

\begin{tabular}{|c|c|c|c|}
\hline Chemical & Formula & Distributor & Purity \\
\hline \multicolumn{4}{|l|}{ Chapter 3} \\
\hline Guaiacol (2-Methoxyphenol) & $\mathrm{C}_{7} \mathrm{H}_{8} \mathrm{O}_{2}$ & Sigma Aldrich & $>98 \%$ \\
\hline Hexanoic acid & $\mathrm{C}_{6} \mathrm{H}_{12} \mathrm{O}_{2}$ & Sigma Aldrich & $99 \%$ \\
\hline n-Undecane & $\mathrm{C}_{11} \mathrm{H}_{24}$ & Sigma Aldrich & $>99 \%$ \\
\hline \multicolumn{4}{|l|}{ Chapter 4} \\
\hline Guaiacol (2-Methoxyphenol) & $\mathrm{C}_{7} \mathrm{H}_{8} \mathrm{O}_{2}$ & Sigma Aldrich & $>98 \%$ \\
\hline \multicolumn{4}{|l|}{ Chapter 5} \\
\hline 1-Methylnaphthalene & $\mathrm{C}_{11} \mathrm{H}_{10}$ & Acros Organics® & $>97 \%$ \\
\hline Cellulose (Avicel PH-101) & $\mathrm{C}_{6} \mathrm{H}_{10} \mathrm{O}_{5}$ & Sigma Aldrich & - \\
\hline Amylopectin from maize & $\mathrm{C}_{6} \mathrm{H}_{10} \mathrm{O}_{5}$ & Sigma Aldrich & - \\
\hline D-(+)-Glucose & $\mathrm{C}_{6} \mathrm{H}_{12} \mathrm{O}_{6}$ & Sigma Aldrich & $>99.5 \%$ \\
\hline \multicolumn{4}{|l|}{ Chapter 6} \\
\hline 1-Methylnaphthalene & $\mathrm{C}_{11} \mathrm{H}_{10}$ & Acros Organics $₫$ & $>97 \%$ \\
\hline Tetralin (1,2,3,4-Tetrahydronaphthalene) & $\mathrm{C}_{10} \mathrm{H}_{12}$ & Sigma Aldrich & $99 \%$ \\
\hline Phenanthrene & $\mathrm{C}_{14} \mathrm{H}_{10}$ & Sigma Aldrich & $98 \%$ \\
\hline Naphthalene & $\mathrm{C}_{10} \mathrm{H}_{8}$ & Sigma Aldrich & $>99 \%$ \\
\hline Toluene & $\mathrm{C}_{7} \mathrm{H}_{8}$ & Sigma Aldrich & $>99,9 \%$ \\
\hline 2,6-Diethylnaphthalene & $\mathrm{C}_{14} \mathrm{H}_{16}$ & Sigma Aldrich & $>97 \%$ \\
\hline Decalin (cis + trans-Decahydronaphthalene) & $\mathrm{C}_{10} \mathrm{H}_{18}$ & Sigma Aldrich & $98 \%$ \\
\hline n-Undecane & $\mathrm{C}_{11} \mathrm{H}_{24}$ & Sigma Aldrich & $>99 \%$ \\
\hline 1-Methoxynaphthalene & $\mathrm{C}_{11} \mathrm{H}_{10} \mathrm{O}$ & Sigma Aldrich & $>98 \%$ \\
\hline 1,2-Dimethoxybenzene & $\mathrm{C}_{8} \mathrm{H}_{10} \mathrm{O}_{2}$ & Sigma Aldrich & $99 \%$ \\
\hline Anisole (Methoxybenzene) & $\mathrm{C}_{7} \mathrm{H}_{8} \mathrm{O}$ & Sigma Aldrich & $>99 \%$ \\
\hline Guaiacol (2-Methoxyphenol) & $\mathrm{C}_{7} \mathrm{H}_{8} \mathrm{O}_{2}$ & Sigma Aldrich & $>98 \%$ \\
\hline Catechol (1,2-Dihydroxybenzene) & $\mathrm{C}_{6} \mathrm{H}_{6} \mathrm{O}_{2}$ & Sigma Aldrich & $>99 \%$ \\
\hline Phenol & $\mathrm{C}_{6} \mathrm{H}_{6} \mathrm{O}$ & Sigma Aldrich & $>99 \%$ \\
\hline 1-Naphthol (1-Hydroxynaphthalene) & $\mathrm{C}_{10} \mathrm{H}_{8} \mathrm{O}$ & Sigma Aldrich & $>99 \%$ \\
\hline Pyrogallol (1,2,3-Trihydroxybenzene) & $\mathrm{C}_{6} \mathrm{H}_{6} \mathrm{O}_{3}$ & Sigma Aldrich & $>99 \%$ \\
\hline Hexanoic acid & $\mathrm{C}_{6} \mathrm{H}_{12} \mathrm{O}_{2}$ & Sigma Aldrich & $99 \%$ \\
\hline Hexanol & $\mathrm{C}_{6} \mathrm{H}_{14} \mathrm{O}$ & Sigma Aldrich & $98 \%$ \\
\hline CGO (Cracked Gas Oil)a & $\mathrm{C}_{1.0} \mathrm{H}_{1.2} \mathrm{~N}_{0,005} \mathrm{~S}_{0,005}$ & $\begin{array}{l}\text { Shell Global Solutions } \\
\text { International B.V. }\end{array}$ & - \\
\hline LCO (Light Cycle Oil) ${ }^{a}$ & $\mathrm{C}_{1.0} \mathrm{H}_{1.4} \mathrm{~N}_{0,01} \mathrm{~S}_{0,01}$ & $\begin{array}{l}\text { Shell Global Solutions } \\
\text { International B.V. }\end{array}$ & - \\
\hline VGO (Vacuum Gas Oil)a & $\mathrm{C}_{1.0} \mathrm{H}_{1.7} \mathrm{~N}_{0,01} \mathrm{~S}_{0,01}$ & $\begin{array}{l}\text { Shell Global Solutions } \\
\text { International B.V. }\end{array}$ & - \\
\hline Hydrowax ${ }^{a}$ & $\mathrm{C}_{1.0} \mathrm{H}_{1.7} \mathrm{~N}_{0,02} \mathrm{~S}_{0,01}$ & $\begin{array}{l}\text { Shell Global Solutions } \\
\text { International B.V. }\end{array}$ & - \\
\hline
\end{tabular}

a More details in the characterization of the refinery streams can be found in the following section (1.1) 


\section{I.I. Characterization of materials and reagents}

Wood composition is shown in Table 3. The Organosolv lignin was characterized with GPC (Figure 1a), Elemental analysis (EA, CHNO analysis), ${ }^{1} \mathrm{H}$ - and ${ }^{13} \mathrm{C}-\mathrm{NMR}$ (Table 4). The refinery streams were also analysed with GPC (Figure 1b and Table 5), EA, ${ }^{1} \mathrm{H}$ - and ${ }^{13} \mathrm{C}-\mathrm{NMR}$ (Table 5).

Table 3. Pine wood composition.

\begin{tabular}{ll} 
Chemical analysis & wt $\%$, dry \\
\hline Cellulose & 35 \\
\hline Hemicellulose & 29 \\
\hline Lignin & 28 \\
\hline Total ash & 1 \\
Elemental analysis & $w t \%$, dry \\
C & 46.6 \\
\hline $\mathrm{H}$ & 6.3 \\
\hline $\mathrm{O}$ (by difference) & 47.0 \\
\hline $\mathrm{N}$ & 0.04 \\
\hline $\mathrm{S}$ & 0.06 \\
\hline
\end{tabular}

Table 4. Characterization results of Organosolv lignin (the range of NMR chemical shifts are defined in sections 7.3 and 7.4).

\begin{tabular}{ll} 
Elemental analysis & $\mathrm{wt} \%$, dry \\
$\mathrm{C}$ & 65.8 \\
\hline $\mathrm{H}$ & 6.3 \\
\hline $\mathrm{O}$ (by difference) & 27.8 \\
\hline $\mathrm{N}$ & 0.1 \\
${ }^{13} \mathrm{C}-\mathrm{NMR}$ & $\mathrm{C} \%$ \\
\hline Paraffin & 14.1 \\
\hline Ethers / alcohols / Acetals & 21.7 \\
\hline Aromatics & 59.0 \\
\hline Carboxyls / carbonyls & 5.2 \\
\hline${ }^{1} \mathrm{H}-\mathrm{NMR}$ & $\mathrm{H} \%$ \\
\hline Paraffin & 28.5 \\
\hline Ethers / alcohols & 44.5 \\
\hline Aromatics & 26.0 \\
\hline Carboxyls / carbonyls & 1.0 \\
\hline
\end{tabular}


Table 5. Characterization results of the refinery streams (the range of NMR chemical shifts for refinery streams are defined in sections 7.3 and 7.4).

\begin{tabular}{|c|c|c|c|c|}
\hline & CGO & LCO & VGO & Hydrowax \\
\hline \multicolumn{5}{|c|}{ Elemental analysis (wt $\%$, dry) } \\
\hline $\mathrm{C}$ & 89.3 & 86.3 & 85.5 & 83.6 \\
\hline $\mathrm{H}$ & 9.0 & 10.3 & 11.9 & 11.5 \\
\hline S (by difference) & 1.1 & 2.9 & 2.0 & 4.1 \\
\hline $\mathrm{N}$ & 0.5 & 0.6 & 0.7 & 0.5 \\
\hline \multicolumn{5}{|l|}{${ }^{13} \mathrm{C}-\mathrm{NMR}(\mathrm{C} \%)$} \\
\hline Paraffins & 35.4 & 39.0 & 77.0 & 89.4 \\
\hline Olefins / Aromatics & 64.6 & 61.0 & 23.0 & 10.6 \\
\hline \multicolumn{5}{|l|}{${ }^{1} \mathrm{H}-\mathrm{NMR}(\mathrm{H} \%)$} \\
\hline Paraffins & 68.8 & 81.6 & 95.1 & 97.2 \\
\hline Olefins & 7.2 & 0.5 & 0.0 & 0.0 \\
\hline Aromatics & 24.0 & 17.9 & 4.9 & 2.8 \\
\hline \multicolumn{5}{|l|}{ GPC } \\
\hline $\mathrm{Mn}(\mathrm{Da})^{\mathrm{a}}$ & 66 & 73 & 228 & 334 \\
\hline $\mathrm{Mw}(\mathrm{Da})^{\mathrm{a}}$ & 117 & 118 & 369 & 562 \\
\hline$\bigoplus_{M}{ }^{b}$ & 1.8 & 1.6 & 1.6 & 1.7 \\
\hline \multicolumn{5}{|l|}{ Other } \\
\hline Boiling range $\left({ }^{\circ} \mathrm{C}\right)$ & $160-370$ & $150-400$ & $200-540$ & $>280$ \\
\hline
\end{tabular}

a Number weighted molecular weight (Mn), Mass weighted molecular weight (Mw).

${ }^{b}$ Dispersity (Đм) was calculated as Mw/Mn. It gives an indication of the distribution or the heterogeneity of the Mw in the refinery stream.
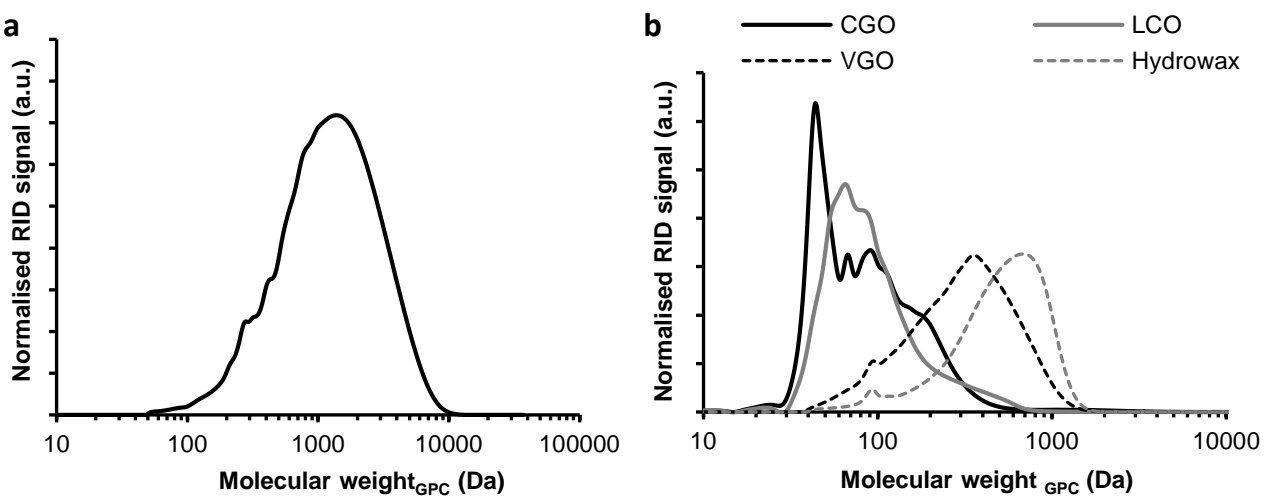

Figure 1. Molecular weight distribution of Organosolv lignin and refinery streams. 


\section{Liquefaction and product recovery}

Pine wood and the liquefaction medium were introduced in a $45 \mathrm{ml}$ autoclave made of Inconel 825 and equipped with a mechanical stirrer (autoclave set-up depicted in Figure 2). The autoclave was then closed tightly and flushed with $\mathrm{N}_{2}$ for oxygen removal and a leak test. Afterwards, the autoclave was automatically submerged into a fluidised sand bed at the reaction temperature and, after liquefaction, moved to a cold water bath to stop the reactions. An initial heating rate of approximately $60^{\circ} \mathrm{C} /$ min allowed to reach $90 \%$ of the reaction temperature within the first 4 minutes and the targeted temperature some 5 min later. The heating time was included in the reaction time. Pressures and temperatures during the experiments were recorded using the Pico Log program.

Three products were obtained after liquefaction, namely gas, bio-crude and solid. After cooling down of the reactor, gas was released, and the free gas volume at atmospheric pressure was measured. A sample was taken for GC analysis. Afterwards, the autoclave was opened and the slurry was filtered to provide a liquid product and a solid filter cake. The reactor was then rinsed with acetone and the wash liquor was filtered over the filter cake. The filter cake was dried at $105^{\circ} \mathrm{C}$ before quantification and analysis. The liquefaction liquid (bio-crude) was composed of two phases after acetone removal: a paste-like product named tar and a lighter product named oil. The total amount of product (solid, bio-crude and gas) typically amounted to more than $90 \mathrm{w} \%$ of the total intake (biomass and solvent). Figure 3 shows a scheme of the procedure followed for the recovery of the liquefaction products.

\section{Recycling experiments}

For the recycling experiments, guaiacol was used as start-up solvent. For the first run, the autoclave (Figure 2) was loaded with pine wood (10 wt\%), water (10 $\mathrm{wt} \%$ ) and guaiacol (80 wt\%), and heated. After reaction, instead of cooling down to room 
temperature, the autoclave was now cooled to $200^{\circ} \mathrm{C}$ and opened to vent off the gas and remove compounds with a low boiling point (referred to as lights in chapter 3), which were condensed and quantified. The autoclave was then cooled down to room temperature and the product bio-crude in solution was obtained via filtration. This bio-crude was then used as solvent medium for the subsequent experiment. A total of 5 experiments ( 4 refills) were done. In the fifth run, $20 \mathrm{wt} \%$ of wood and $10 \mathrm{wt} \%$ of water were loaded.

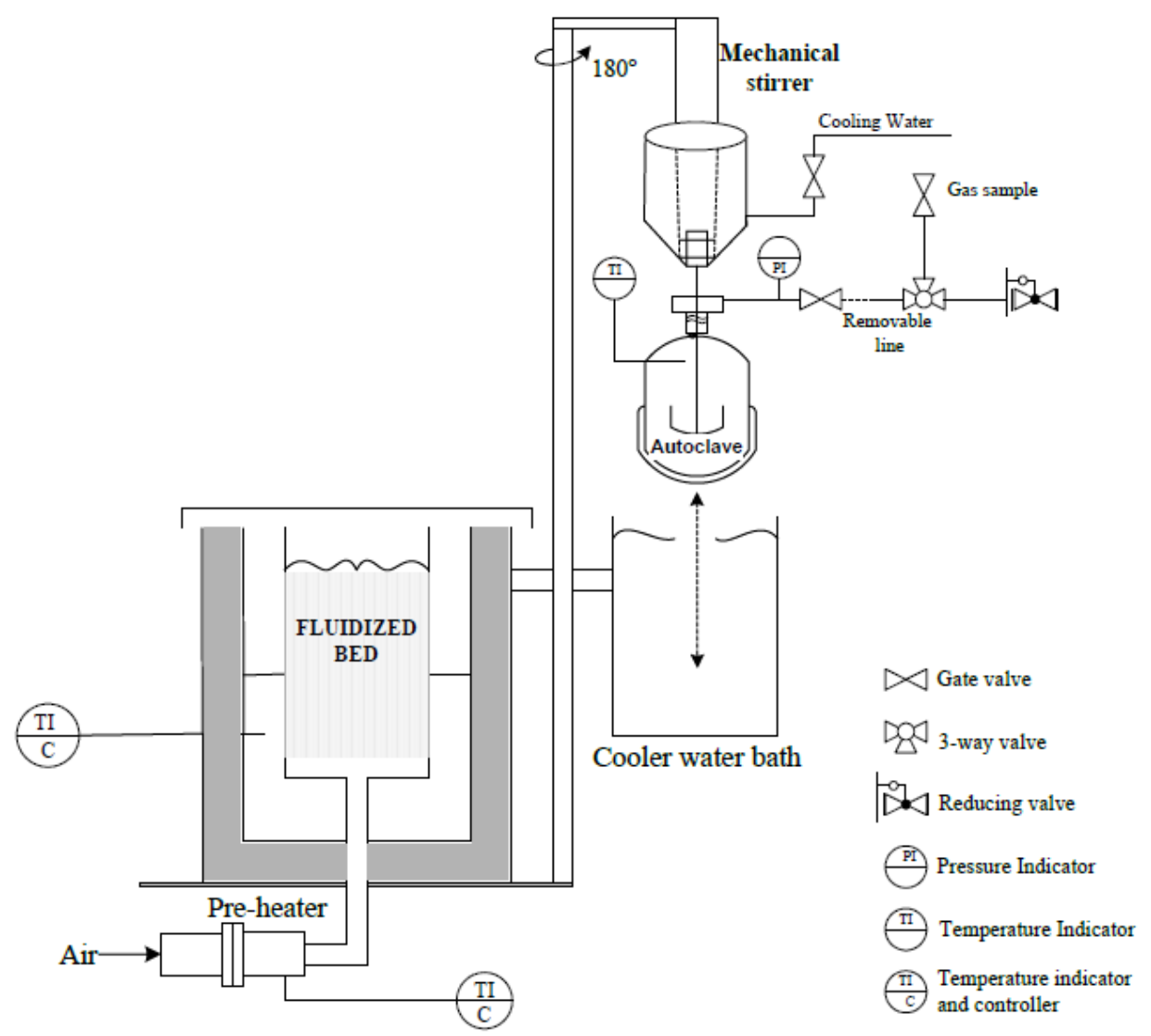

Figure 2. Scheme of the liquefaction set-up. 


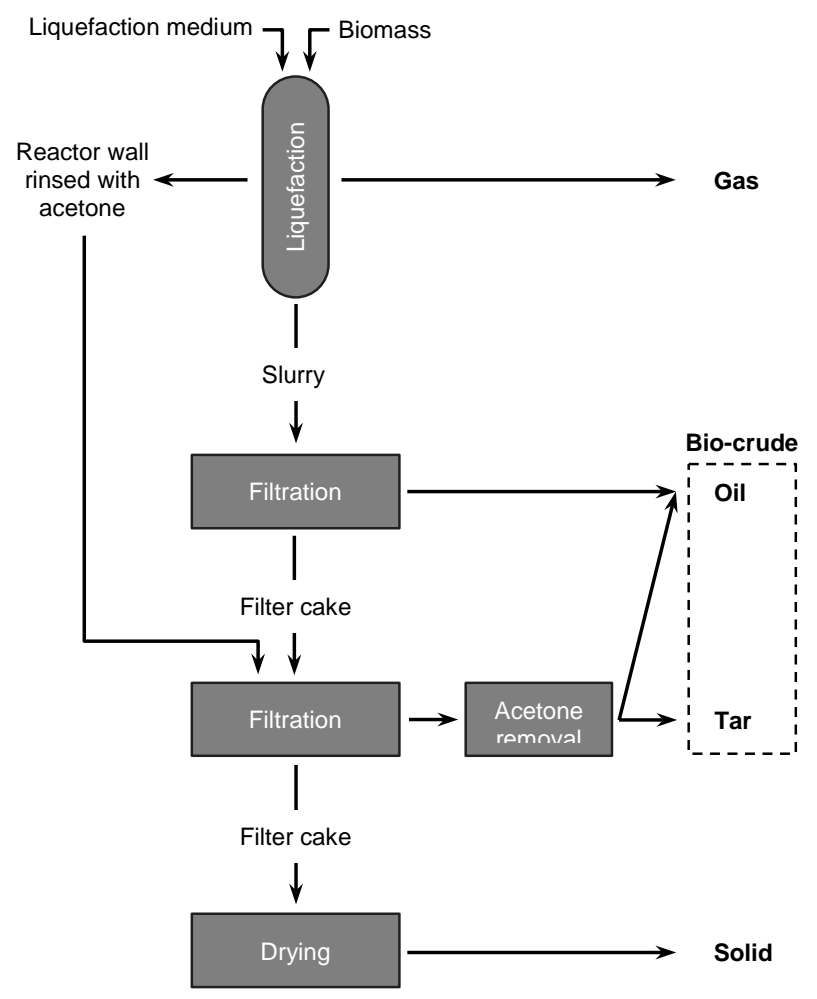

Figure 3. Procedure followed for the recovery of the liquefaction products.

\section{Fast pyrolysis}

Pyrolysis oil was produced in a continuous fluidized bed reactor that used sand as fluidized bed particles. The vapours formed were directed to a condenser system composed by two counter-current spray condensers and an intensive cooler placed in series. The non-condensable gases were collected and analysed for mass balance closure. The char produced was calculated by subtracting the initial amount of sand to the total solid residue. The condensates were mixed to obtain the fast pyrolysis oil. A detailed description of the fast pyrolysis procedure and setup can be found in [2]. Characterization results of the pyrolysis oil can be found in chapter 4 . 


\section{Fractionation}

To do the GPC preparative fractionation, the sample was dissolved in THF and filtered through a $0.45 \mu \mathrm{m}$ syringe filter. A multidraw kit allowed the injection of 1.5 $\mathrm{ml}$ of sample into an Agilent Technologies 1200 system composed of a pre-column (PLgel 25x25mm), a column (PLgel 300x25mm with $5 \mu \mathrm{m}, 500 \mathrm{~A}$ ) and a fraction collector. The fractionation was performed at room temperature for 50 minutes, and various bio-crude fractions were collected in intervals of one minute. The THF of the obtained fractions was evaporated under vacuum $(10-20 \mathrm{mbar})$ and $a 5^{\circ} \mathrm{C}$ to obtain the isolated bio-crude fractions.

Figure 4 shows the fractions obtained after GPC preparative fractionation of biocrude. More details about GPC preparative fractionation can be found in chapter 4 and in the supporting information (section B).

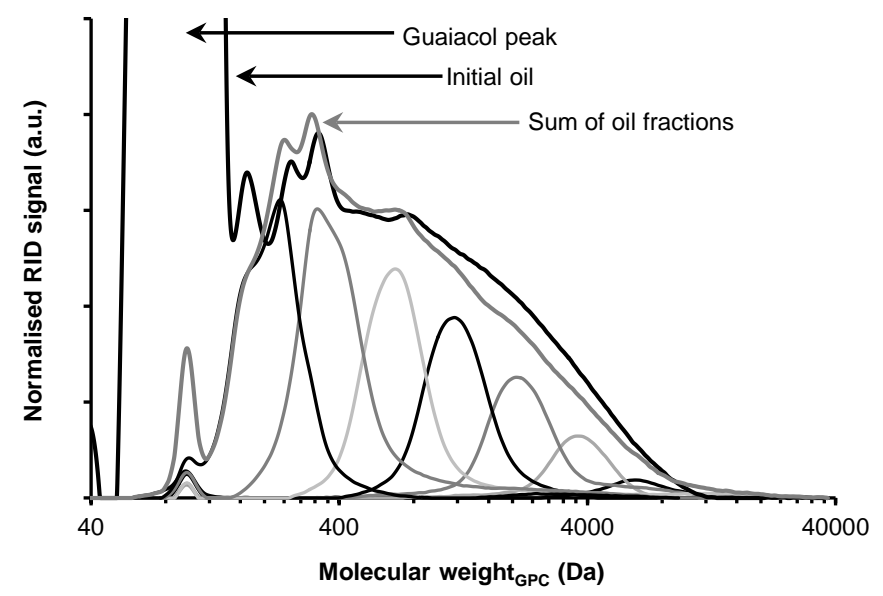

Figure 4. GPC chromatograms for a bio-crude fractionation. MwGPC distribution of a liquefaction liquid product (bio-crude with the solvent), some of its fractions obtained by GPC fractionation and the sum of all these obtained fractions. The fractionated liquid product was obtained after liquefaction of wood (10 wt\%) in guaiacol (90 wt\%) at $300^{\circ} \mathrm{C}$ during 30 minutes. 


\section{Product definition and calculation}

\section{I. Product yields}

For both liquefaction and pyrolysis experiments, gas refers to all the noncondensable gases, bio-crude refers to the acetone soluble compounds and solid residue includes all the acetone insoluble compounds. All the yields presented in this paper (with the exception of the recycling experiments) are expressed in carbon percentage $(\mathrm{C} \%)$ and are calculated using the following equations:

Gas yield $(\mathrm{C} \%)=\frac{\text { mole of carbon in gas } * 12}{\text { gram of wood input } * \text { carbon content of wood }} * 100$

Equation 1

Solid yield $(\mathrm{C} \%)=\frac{\text { gram of solid } * \text { carbon content of solid }}{\text { gram of wood input } * \text { carbon content of wood }} * 100$

Equation 2

Biocrude yield $(\mathrm{C} \%)=100-$ Gas yield - Solid yield

(liquefaction)

Equation 3

Biocrude yield $(\mathrm{C} \%)=\frac{\text { gram of biocrude } * \text { carbon content of biocrude }}{\text { gram of wood input } * \text { carbon content of wood }} * 100 \quad$ (pyrolysis) $\quad$ Equation 4

To calculate the gas yield, the volume percentages measured with a Micro-GC (section 7.7) were converted into mole percentages using the ideal gas law. Once the number of moles of each gas component was known, the total grams of carbon in the gas could be easily calculated.

Carbon percentage of the liquefaction solids and the pyrolysis oils was directly analysed with the EA. Unfortunately, no EA could be performed for the solid residue of the pyrolysis experiment since it was mixed with the sand used in the fluidized bed. Therefore, the carbon content of the pyrolysis solid was obtained from literature [3], and corresponded to the char produced during pyrolysis of pine shave at $500^{\circ} \mathrm{C}$. 
Due to the nature of the recycling experiments, product yields had to be reported in weight percentage. The solid and gas yields were calculated dividing the grams of solid and gas produced by the initial grams of wood. Bio-crude yield was calculated by difference (biocrude yield $=100-$ solid yield - gas yield).

\subsection{Heavy and light bio-crude}

In the following chapters, we use the terms 'Vacuum Residue' (VR) and 'Distillate' to refer, respectively, to the heavy and light species present in the bio-crude or liquid product. VR is defined as all the components in the liquid with an MwGPC higher than $1000 \mathrm{Da}$, and Distillate comprises the liquid fraction with MwGPC lower than $1000 \mathrm{Da}$ (excluding the liquefaction solvent, Figure 5). The percentages of VR and Distillate are calculated using the GPC chromatogram of the studied liquid. The RID signal is plotted versus the elution time, and the area corresponding to the VR (MwGPC $>1000 \mathrm{Da}$ ) or to the Distillate (MwGPC < 1000; excluding the liquefaction solvent peak) is divided by the total area (see Equation 5 and Equation 6). The cut point between the solvent and the Distillate is defined as the minimum seen in GPC trace just after the solvent peak. It varies from 72 to $250 \mathrm{Da}$ depending on the solvent used as liquefaction media. A visual inspection of the various GPC traces shown in this thesis reveals that the variation in cut point is bringing only a modest uncertainty to the overall integral of the bio-crude part of the GPC trace.

VR fraction $(\%)=\frac{\text { RID area corresponding to } \mathrm{Mw}_{\mathrm{GPC}}>1000 \mathrm{Da}}{\text { Total RID area (excluding solvent peak) }}$

Equation 5

Distillate fraction $(\%)=\frac{\mathrm{RID} \text { area corresponding to } \mathrm{Mw}_{\mathrm{GPC}}<1000 \mathrm{Da} \text { (excl. solvent peak) }}{\text { Total RID area (excluding solvent peak) }}$

Equation 6

The yields of VR and Distillate are calculated using the following equations: 


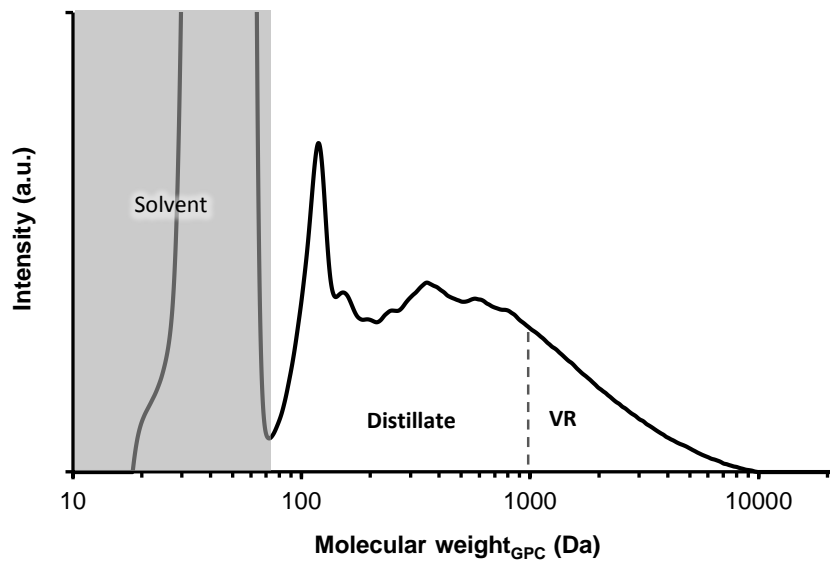

Figure 5. Scheme of the procedure followed for the quantification of heavies in the bio-crude.

\subsection{Bio-crude composition and quality}

Various parameters have been used to report the composition and the quality of the bio-crude.

In chapters 4 and 5 , quantitative ${ }^{13} \mathrm{C}-\mathrm{NMR}$ and ${ }^{1} \mathrm{H}-\mathrm{NMR}$ are used to determine the yield or content of aromatic, paraffinic or oxygenated species in the liquid products or organosolv lignin. Equation 9 and Equation 10 are used to calculate the yield of liquid aromatic species, and the aromatics with lignin origin respectively. 
$\operatorname{Aromatics}_{\text {lignin }}(\mathrm{C} \%)=\frac{\text { Lignin content of wood } * \text { Carbon content of lignin } * \text { Aromatic content of lignin }}{\text { Carbon content of wood }} * 100$

These equations are expressed in $\mathrm{C} \%$ and, therefore, the aromatic content is determined by ${ }^{13} \mathrm{C}-\mathrm{NMR}$. Equivalent equations could be used to make the calculations in $\mathrm{H} \%$ (using ${ }^{1} \mathrm{H}-\mathrm{NMR}$ results) or to calculate the yields of paraffinic or oxygenated species.

Effective $\mathrm{H} / \mathrm{C}$ ratio $\left(\mathrm{H} / \mathrm{C}_{\text {eff }}\right)$ and Higher heating value $(\mathrm{HHV})$ were used as indicators of bio-crude quality in chapters 4 and 5. The $\mathrm{H} / \mathrm{C}_{\text {eff }}$ gives an estimation of the hydrogen left in the product once all the oxygen is removed as water (Equation 11). The HHV is the amount of heat produced when a fuel is completely combusted and all the combustion products are cooled down to the initial temperature of the fuel. By returning the final products to the pre-combustion temperature, the latent heat of vaporization of the water steam is included in the HHV. The HHV is calculated with the Reed's formula [4] (Equation 12).

$\mathrm{H} / \mathrm{C}_{\text {eff }}=\frac{\text { mole hydrogen }-2 * \text { mole oxygen }}{\text { mole carbon }}$

Equation 11

$\mathrm{HHV}(\mathrm{MJ} / \mathrm{kg})=0.341 *$ carbon wt $\%+1.322 *$ hydrogen wt $\%-0.12 *$ oxygen wt $\%$

Equation 12

\subsection{Calculation of solubility parameters}

The Hildebrand solubility parameter $(\delta)$ of a component is defined as the square root of the cohesive energy density. It represents the energy needed to evaporate an elementary volume of this solvent, and it is generally used to study the interaction 
between materials. The Hildebrand value can be calculated with the following equation [5]:

$\delta=\sqrt{\frac{\Delta \mathrm{H}_{v}-\mathrm{RT}}{\mathrm{Vm}}}$

Equation 13

where $\Delta \mathrm{H}_{\mathrm{v}}$ is the enthalpy of vaporization, $\mathrm{R}$ the ideal gas constant, $\mathrm{T}$ the temperature and $\mathrm{V}_{\mathrm{m}}$ the molar volume of the pure solvent.

Materials with similar Hildebrand values are likely to be miscible. However, this parameter does not consider certain type of associations such as polar interaction or hydrogen-bonding. As a consequence, occasionally, two materials might have similar Hildebrand values but be immiscible. One solution to this problem was formulated by Hansen et al. [6]. They proposed that the total energy of vaporization consists of several individual parts, and divided the Hildebrand solubility parameter into three partial solubility parameters, namely dispersion $(\delta \mathrm{D})$, polarity $\left(\delta_{\mathrm{P}}\right)$ and H-bonding $\left(\delta_{\mathrm{H}}\right)$. The relation between the Hildebrand and the partial Hansen solubility parameters is described by equation [6]:

$\delta=\sqrt{\delta_{D}^{2}+\delta_{P}^{2}+\delta_{H}^{2}}$

Equation 14

The solubility parameters of solvent mixtures can be calculated with the following equation [6]:

$\delta_{(\mathrm{D}, \mathrm{P}, \mathrm{H})}=\phi_{1} * \delta_{(\mathrm{D}, \mathrm{P}, \mathrm{H}) 1}+\phi_{2} * \delta_{(\mathrm{D}, \mathrm{P}, \mathrm{H}) 2}+\phi_{3} * \delta_{(\mathrm{D}, \mathrm{P}, \mathrm{H}) 3}$

Equation 15

being $\phi_{1,2,3}$ the volume fractions of each solvent. 
The solubility radius ( $\mathrm{Ra}$ ) around a solute defines the combination of $\delta_{\mathrm{D}}, \delta_{\mathrm{p}}$ and $\delta_{\mathrm{H}}$ of solvent that offer similar interaction with the solute. The lower the Ra, the higher the interaction. Ra can be calculated with the equation [6]:

$R a=\sqrt{4 *\left(\delta_{\mathrm{D} 2}-\delta_{\mathrm{D} 1}\right)^{2}+\left(\delta_{\mathrm{P} 2}-\delta_{\mathrm{P} 1}\right)^{2}+\left(\delta_{\mathrm{H} 2}-\delta_{\mathrm{H} 1}\right)^{2}}$

Equation 16

The Hildebrand and Hansen partial solubility parameters used in this work were obtained from [6]. According to [6], Hansen solubility parameters of complex components such as cellulose and lignin were calculated by studying their solubility or degree of swelling in a series of well-defined solvents. The Hansen parameters of the refinery streams were estimated by comparing each refinery stream average structure with known molecules (see section 1.1).

\section{Characterization techniques}

\section{I. Gel Permeation Chromatography (GPC)}

The Mw distribution of liquids and organosolv lignin was determined with GPC. The analyses were performed with a system from Agilent Technologies 1200 composed by three columns placed in series $(7.5 \times 300 \mathrm{~mm}$, particle size $3 \mu \mathrm{m})$ packed with a highly crosslinked polystyrene-divinylbenzene copolymer gel (Varian, PLgelMIXED-bed E), a refractive index-detector (RID) and an Variable wavelength detector (VWD) operated at $254 \mathrm{~nm}$. Bio-crudes were dissolved in THF and filtered through a $0.45 \mu \mathrm{m}$ syringe filter. Then, $20 \mu \mathrm{l}$ of sample were injected into the system operated at $40^{\circ} \mathrm{C}$ and with a flow of $1 \mathrm{ml} / \mathrm{min}$ of THF. Measurements lasted 40 minutes. The calibration line made with polystyrene standards of several molecular weights (162-29510 g/mole) was used for the conversion of elution volume to molecular weight (MwGPC). 


\subsection{Fourier Transform Infrared Spectroscopy (FTIR)}

Qualitative FTIR analyses were performed with a Fourier Transform Infrared Spectrophotometer from Bruker equipped with an Attenuated total reflection system (ATR) and a deuterated triglycine sulphate detector (DTGS). Absorbance was measured in the range 650 to $4000 \mathrm{~cm}^{-1}$ with an spectral resolution of $4 \mathrm{~cm}^{-1}$, and 16 scans were performed. Afterwards, the spectra were baseline corrected and normalized.

\section{3. ${ }^{13} \mathrm{C}$ Nuclear Magnetic Resonance $\left({ }^{13} \mathrm{C}-\mathrm{NMR}\right)$}

Quantitative ${ }^{13} \mathrm{C}-\mathrm{NMR}$ measurements were performed with a Bruker $600 \mathrm{MHz}$ Avance II NMR spectrometer. Samples were prepared by dissolving approximately $0.25 \mathrm{~g}$ of (solvent-free) sample and $0.024 \mathrm{~g}$ of $\mathrm{Cr}(\mathrm{AcAc}) 3$ (relaxation agent) in $0.7 \mathrm{ml}$ of deuterated DMSO. Measurements were performed at $40^{\circ} \mathrm{C}$ using the 'inverse gate decoupling' method with 5 seconds of relaxation time and 5000 scans. The DMSO signal was used as internal reference.

Manual integration of the spectra was performed with MestReNova LITE software (version 5.2.5-5780). When present, solvent peaks (DMSO, acetone or THF) were manually integrated and subtracted from the corresponding region. The integration regions were defined according to Ben et.al and Ingram et.al $[7,8]$ and are shown in Table 6.

${ }^{13} \mathrm{C}-\mathrm{NMR}$ analyses of refinery streams were performed with a Bruker $400 \mathrm{MHz}-$ Ascend NMR spectrometer using 'inverse gate decoupling' with 5 seconds of relaxation time and 1024 scans. Samples were prepared as described previously. However, Hydrowax was not soluble in pure DMSO and, therefore, it was dissolved a mixture of cyclohexane and deuterated DMSO. 
Table 6. Integration regions for ${ }^{13} \mathrm{C}-\mathrm{NMR}$.

\section{Functional group Integration region Examples}

Alkanes

(aliphatic C-C bond)

$1-54 \mathrm{ppm}$

$\mathrm{H}_{3} \mathrm{C}-\mathrm{R}$

${ }_{\mathrm{R}} \stackrel{\mathrm{H}_{\mathrm{C}}}{\mathrm{H}_{\mathrm{R}^{\prime}}}$

$\left.\right|_{\mathrm{R}^{\prime}} ^{\mathrm{C}} \mathrm{H}_{\mathrm{R}^{\prime \prime}}^{\mathrm{R}}$

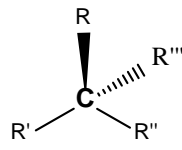

Aliphatic alcohol/

ethers/acetals

54-105 ppm

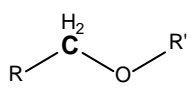

(methoxy groups)

$55.2-60.8 \mathrm{ppm}$

${ }^{\mathrm{H}_{3} \mathrm{C}} \backslash_{\mathrm{O}}{ }^{\mathrm{R}}$

\begin{tabular}{llll}
\hline (acetals) & $84-105 \mathrm{ppm}$ & $105-166 \mathrm{ppm}$ \\
Aromatics & $166-210 \mathrm{ppm}$
\end{tabular}

\section{4. 'H Nuclear Magnetic Resonance ('H-NMR)}

For ${ }^{1} \mathrm{H}-\mathrm{NMR}$ quantitative analyses, approximately $0.1 \mathrm{~g}$ of sample was dissolved in $0.7 \mathrm{ml}$ of deuterated acetone. Hydrowax was dissolved a mixture of cyclohexane and deuterated acetone. The analyses were performed with a Bruker $400 \mathrm{MHz}$-Ascend NMR spectrometer. A relaxation time of 2 seconds and 128 scans were used. Integration of the spectra was performed following the same procedure described for ${ }^{13} \mathrm{C}-\mathrm{NMR}$.

The assignation of the peaks for ${ }^{1} \mathrm{H}-\mathrm{NMR}$ was based on the papers from Ingram et.al and Mullen et.al $[8,9]$. Integration regions are shown in Table 7. When visible, the broad band corresponding to the hydrogen in alcohol groups appeared under $3 \mathrm{ppm}$ (region of the Aliphatic $\alpha$ to an unsaturation or an heteroatom). Only three 
integration regions were defined for the refinery streams, namely alkanes (0-4.5 ppm), olefins (4.5-6.5 ppm) and aromatics (6.5-9.0 ppm) [10, 11].

Table 7. Integration regions for ${ }^{1} \mathrm{H}-\mathrm{NMR}$.

\section{Functional group Integration region Examples}

Alkanes

$0.5-3.0 \mathrm{ppm}$

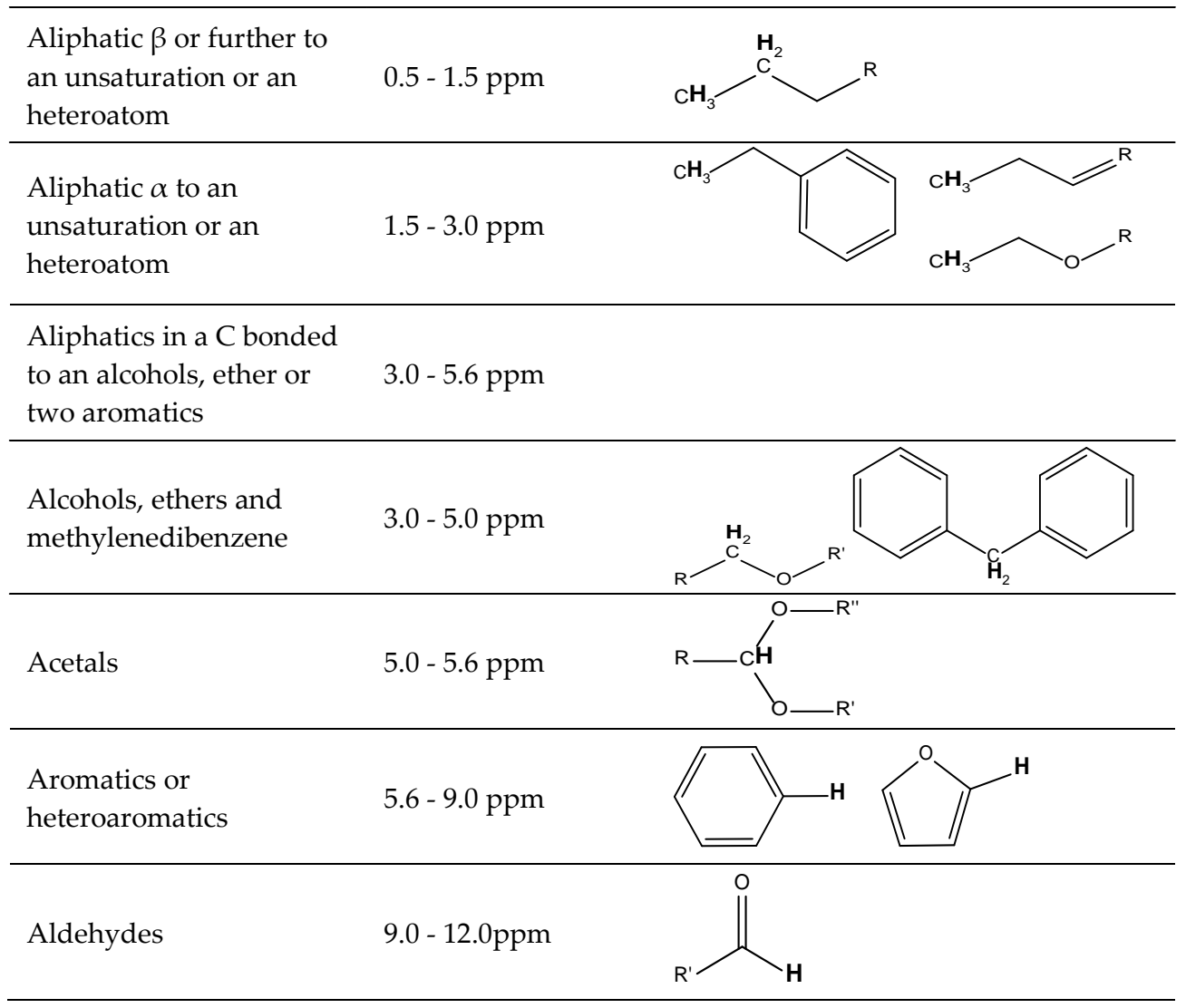

\subsection{Elemental Analysis (EA)}

The carbon, hydrogen and nitrogen content of solid and liquid products was determined with an Elemental Analyser Inter Science Flash 2000. The oxygen (sulphur in refinery streams) content of the samples was calculated by difference. 


\subsection{Micro Carbon Residue Test (MCRT)}

The carbon residue was measured with a Micro Carbon Residue tester ACR-M3 Zematra.

\subsection{Gas analysis}

Gas analyses were performed with a Micro-Gas Chromatograph (Variant CP-4900) equipped with two columns. The first column (Molsieve $5 \mathrm{~A}(10 \mathrm{~m})$ ) detected $\mathrm{H}_{2}, \mathrm{O}_{2}$, $\mathrm{N}_{2}, \mathrm{CH}_{4}$ and $\mathrm{CO}$, while the second column (PPQ (10 m)) detected $\mathrm{CO}_{2}, \mathrm{C}_{2} \mathrm{H}_{4}, \mathrm{C}_{2} \mathrm{H}_{6}$, $\mathrm{C}_{3} \mathrm{H}_{6}$ and $\mathrm{C}_{3} \mathrm{H}_{8}$. Helium was used as a carrier gas.

\subsection{Gas Chromatography-Mass Spectrometry (GC-MS)}

GC-MS analyses were performed with a gas chromatograph equipped with a mass spectrometer (GC 7890A MS 5975C, Agilent Technologies). Samples were first dissolved in acetone ( $5 \mathrm{wt} \%$ ) and filtered through a $0.2 \mu \mathrm{m}$ Whatman filter. Then, 1 $\mu \mathrm{L}$ of sample was injected into the injector port set at $250^{\circ} \mathrm{C}$ and with a split ratio of 20:1. The capillary column (Varian CP9154, $60 \mathrm{~m}, 0.25 \mathrm{~mm}$ ) was packed with 0.25 $\mu \mathrm{m}$ of $14 \%$ cyanopropyl-phenyl and 86\% PDMS, and operated at a constant Helium flow of $2 \mathrm{ml} / \mathrm{min}$ for $106 \mathrm{~min}$. Oven temperature was set at $45^{\circ} \mathrm{C}$ for $4 \mathrm{~min}$, then increased at a rate of $3^{\circ} \mathrm{C} / \mathrm{min}$ and finally maintained at $280^{\circ} \mathrm{C}$ for $20 \mathrm{~min}$. The mass spectrometer was operated under electron ionization mode $(70 \mathrm{eV})$, with a frequency of $1 \mathrm{scan} / \mathrm{s}$, and detected a m/z range between 15 and 500. A spectral library (NIST Mass Spectral Library Version 08) was used to identify the detected compounds.

\subsection{Scanning Electron Microscopy (HR-SEM)}

The morphology of chars was studied with Scanning Electron Microscopy (Analysis Zeiss MERLIN HR-SEM). The system was equipped with a Hot Field Emission Gun 
and a detector HE-SE2. Measurements were done using a voltage of $0.85 \mathrm{kV}$ and a prove current of 15-60 pA.

\section{I 0. Karl-Fischer titration (KFT)}

The water concentration in the liquid was determined by Karl-Fischer titration using a Metrohm 787 KF Titrino. Hydranal Composite 5 was used as titrant and a solution of ethanol:dichloromethane (3:1 in volume) was used as solvent.

\section{II. Viscosity measurements}

Viscosity of the oils was measured with a rotary viscometer Brookfield DV-E at $30^{\circ} \mathrm{C}$. 


\section{References}

1. Huijgen, W.J.J., A.T. Smit, P.J. de Wild, and H. den Uil, Fractionation of wheat straw by prehydrolysis, organosolv delignification and enzymatic hydrolysis for production of sugars and lignin. Bioresource Technology, 2012. 114(0): p. 389-398.

2. Westerhof, R.J.M., D.W.F. Brilman, M. Garcia-Perez, Z. Wang, S.R.G. Oudenhoven, W.P.M. van Swaaij, and S.R.A. Kersten, Fractional Condensation of Biomass Pyrolysis Vapors. Energy \& Fuels, 2011. 25(4): p. 1817-1829.

3. Ahmad, M., A.U. Rajapaksha, J.E. Lim, M. Zhang, N. Bolan, D. Mohan, M. Vithanage, S.S. Lee, and Y.S. Ok, Biochar as a sorbent for contaminant management in soil and water: A review. Chemosphere, 2014. 99(0): p. 19-33.

4. Domalski, E.S., T.L.J. Jobe, and T.A. Milne, Thermodynamic data for biomass conversion and waste incineration. 1987, American Society of Mechanical Engineers, New York.

5. Hildebrand, J. and R.L. Scott, The Solubility of Nonelectrolytes. 3rd ed, ed. A.C. Society. 1950, New York: Reinhold.

6. Hansen, C.M., Hansen solubility parameters: A user's handbook. 2000, United States of America: CRC Press LLC.

7. Ben, H. and A.J. Ragauskas, NMR Characterization of Pyrolysis Oils from Kraft Lignin. Energy \& Fuels, 2011. 25(5): p. 2322-2332.

8. Ingram, L., D. Mohan, M. Bricka, P. Steele, D. Strobel, D. Crocker, B. Mitchell, J. Mohammad, K. Cantrell, and C.U. Pittman, Pyrolysis of Wood and Bark in an Auger Reactor: Physical Properties and Chemical Analysis of the Produced Bio-oils. Energy \& Fuels, 2007. 22(1): p. 614-625.

9. Mullen, C.A., G.D. Strahan, and A.A. Boateng, Characterization of Various Fast-Pyrolysis Bio-Oils by NMR Spectroscopyt. Energy \& Fuels, 2009. 23(5): p. 2707-2718.

10. Sarpal, A.S., G.S. Kapur, S. Mukherjee, and A.K. Tiwari, PONA analyses of cracked gasoline by 1 H NMR spectroscopy. Part II. Fuel, 2001. 80(4): p. 521-528.

11. Burri, J., R. Crockett, R. Hany, and D. Rentsch, Gasoline composition determined by $1 H$ NMR spectroscopy. Fuel, 2004. 83(2): p. 187-193. 


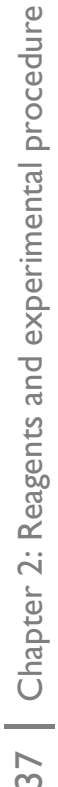




\section{Chapter 3}

Solvent, process parameter and recycle bio-crude screening 
Liquefaction of lignocellulosic biomass was studied for the production of liquid (transportation) fuels. The process concept used a product recycle as liquefaction medium and produced a bio-crude that can be co-processed in a conventional oil refinery. This all was done at medium temperature $\left(\sim 300{ }^{\circ} \mathrm{C}\right)$ and pressure $(\sim 60$ bar). Solvent screening experiments showed that oxygenated solvents are preferred as they allow high bio-crude (up to $93 \%$ on carbon basis) and low solid yields ( 1-2 $\%$ on carbon basis) and, thereby outperform liquefaction of biomass in compressed water and biomass pyrolysis. The following solvent ranking was obtained: guaiacol $>$ hexanoic acid >> n-undecane. The usage of wet biomass resulted in higher biocrude yields than dry biomass. However, it also resulted in higher operating pressure, which would make the process more expensive. Refill experiments were also performed to evaluate the possibility to recycle the bio-crude as liquefaction medium. The recycle bio-crude appeared to be very effective in liquefying the biomass, even surpassing the start-up solvent guaiacol, but became increasingly heavy and more viscous after each refill and eventually showed a molecular weight distribution which resembled that of refinery vacuum residue.

This chapter has been published as:

van Rossum, G.; Zhao, W.; Castellvi Barnes, M.; Lange, J.-P. and Kersten, S. R. A.; Liquefaction of Lignocellulosic Biomass: Solvent, Process Parameter, and Recycle Oil Screening. ChemSusChem, 2013, 7(1): p. 253-259. 


\section{Introduction}

One of the various possible routes to convert (lignocellulosic) biomass into bio-crude is the liquefaction, which produces a bio-crude with moderate oxygen content, for example, $6-30 \mathrm{wt} \%$ [1, 2] versus 35-40 $\mathrm{wt} \%$ for pyrolysis bio-crude [3] and 40-50 $w t \%$ for the initial lignocellulose. The process temperature is milder than in pyrolysis (up to $\sim 400{ }^{\circ} \mathrm{C}$ ) but the use of solvent often results in higher pressures (up to $\sim 250$ bars if water is used as solvent). An overview of literature on biomass liquefaction is given in Table A1 (Supporting information). Solvent selection is very important to obtain high liquefaction bio-crude yields. Polar solvents usually perform better than apolar solvents. Combined bio-crude and gas yields up to $99 \mathrm{wt}$ $\%$ have been reported for liquefaction with and without the use of a homogeneous and/or heterogeneous catalyst. From a commercial point of view, solvent cost should be minimized. Hence the solvent should either be cheap and easily recoverable, be produced within the process and/or be co-processed with the bio-crude to the end products. From as early as the 1970's, processes have been developed to pilot scale based on two solvent types, namely water and wood liquefaction recycle bio-crude. Comprehensive overviews of direct liquefaction processes have been given by Behrendt et. al. [4] and Elliot et. al. [5]. These processes all had as main drawbacks that the product was not suitable for final upgrading to transportation fuels and/or that process conditions were too harsh to become economically viable. The processes with recycle bio-crude resulted mainly in a product bio-crude that was too viscous, and the use of water and/or reducing gas led to very high operating pressures (> 150 bar). Therefore processes have been developed up to pilot scale but have not reached commercialization. Hence, from a process and product quality point of view, further progress is still required. Recent efforts $[6,7]$ and this study have tried to gain more insight in the desired solvent properties and operating conditions for biomass liquefaction, in which high bio-crude yields are obtained with a moderate viscosity. In this chapter, we reported our initial efforts to improve the economics of biomass liquefaction [7]. Our approach was based on the idea that the process should: 
- operate without a catalyst to avoid contamination of the resulting bio-crude, which inevitably leads to elevated operating temperature,

- operate at mild pressure by avoiding reactive gases such as $\mathrm{CO}$ or $\mathrm{H}_{2}$ and by using a high-boiling solvent,

- use an inexpensive solvent that does not require separation from the bio-crude, for example, by using a fraction of the bio-crude itself as solvent or by using a cheap refinery stream. Although inexpensive, water disqualified by its high vapour pressure at elevated temperature.

Hence, our research focused on a limited number of solvents that show fairly high boiling points $\left(200^{\circ} \mathrm{C}\right)$ and are model components for a bio-crude fraction or refinery streams, namely guaiacol, hexanoic acid and n-undecane. Guaiacol represents lignin degradation products, hexanoic acid represents carboxylic acids that are formed through cellulose and hemicellulose decomposition, and nundecane represents a refinery stream. As the liquefaction appeared to proceed best with bio-crude model components, refill experiments were performed to simulate the recycle of bio-crude as the liquefaction medium. While the research was underway, another research group appeared to follow a similar approach to biomass liquefaction [6].

\section{Result and discussion}

\section{I.Solvent screening}

During liquefaction, biomass is depolymerized and broken down into smaller segments. The type of solvent used is of paramount importance for the obtainable yields of solid residue, bio-crude and gas. The solvent can (partly) dissolve original biomass polymers and its initial fragments, stabilize and dilute the products formed and also act as a reactant. The effect of solvent selection is clearly illustrated in Figure 1a, in which pine wood liquefaction results are shown as carbon distribution to solid, 
bio-crude and gas for the three different solvents, namely guaiacol, hexanoic acid, and undecane. Both dry and wet (10 wt $\%$ on total feed basis) wood were used. In particular, wet biomass is of special interest as drying biomass is expensive and preferably minimized for each application. Additionally, a run in water and a run without any liquid (solvent-free/pyrolysis) were performed for comparison. The solid does not only have to be a product in the form of char/coke but can also be unconverted wood. The solvent type appeared to have a very big impact on the carbon distribution of the products. Aromatic and/or oxygenated solvents generally performed better than an aliphatic hydrocarbon. According to carbon converted to bio-crude, the solvent performance can be ranked as follows: guaiacol $\approx$ water $>$ hexanoic acid $>>$ undecane $>>$ solvent-free/pyrolysis (Figure 1a)). A quick look at common solvent parameters (see table A4, Supporting information) did not reveal a clear correlation between the solvent and its effectiveness in liquefaction. Clearly, a more extensive set of solvents would be needed to unravel such correlation but this was not the purpose of the present study. Wet wood (50\% moisture, reported as 10 $\mathrm{wt} \%$ wood, $10 \mathrm{w} \%$ water and $80 \mathrm{wt} \%$ of solvent) led to a higher bio-crude yield for every solvent used (Figures 1a and $1 \mathrm{~b}$ ). As a downside, the operating pressures where significantly increased (from 32 - 56 bar without water to $60-121$ bar with water). The addition of water was further investigated with guaiacol and a minimum char yield was observed for intermediate water/guaiacol ratios between 1:4 and 4:1 (Figure 1b). This showed that a mixture of solvents can lead to an added beneficial effect.

The conversions and effectiveness of solvents observed here were generally in agreement with data reported for similar solvents. Phenol, which is chemically similar to guaiacol, shows a very good performance for biomass liquefaction [8-11]. Hydrocarbon solvents like tetralin [12, 13], toluene [14] and "Shellsol" [15] give somewhat lower bio-crude yield. If water is used as a co-solvent, almost no solids and little gas are obtained for guaiacol (Figure 1) and phenol [9], which shows that the addition of water as a co-solvent is generally favourable. Water as a pure solvent 
(e.g. hydrothermal liquefaction of wet biomass) generally results in lower bio-crude yields (up to $58 \mathrm{wt} \%$ ) $[2,16]$.

a

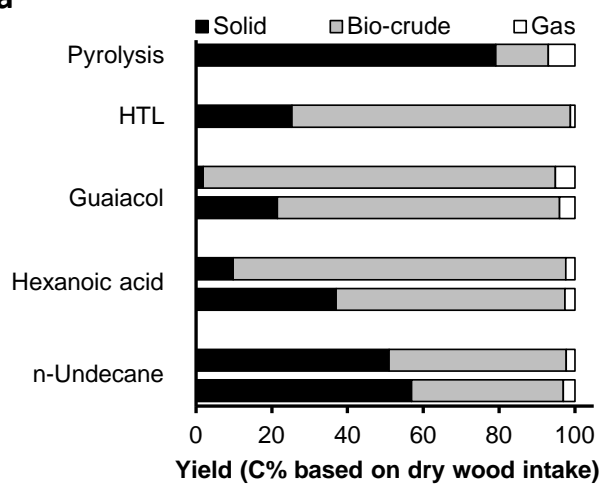

b

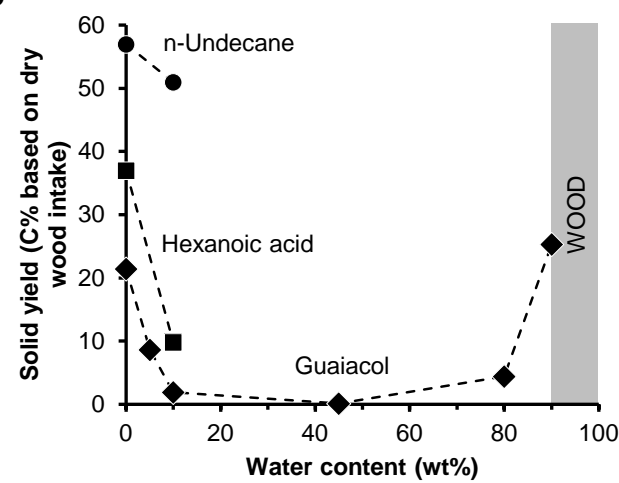

Figure 1. (a) Products distribution obtained after liquefaction of wood (10 wt $\%)$ in guaiacol, hexanoic acid, and undecane with and without the addition of $10 \mathrm{wt} \%$ of water. The product distribution of wood pyrolysis (solvent-free) and wet wood liquefaction (HTL) is also shown. (b) Solid yields plotted versus water content using four different solvents. The temperature was $\sim 300^{\circ} \mathrm{C}$ with a reaction time of $30 \mathrm{~min}$. Details of the experiments can be found in Table A2 of the Supporting information.

\subsection{Product quality}

The bio-crude produced from the liquefaction of biomass still needs further processing if transportation fuels are desired. Co-processing with fossil crude oil (fractions) seems an attractive way for upgrading as use can be made of an already installed infrastructure and known technology. Promising results in this field have been obtained for a different route in which, on lab scale, biomass is added to a fluid catalytic cracking (FCC) unit by pyrolysis and hydrotreatment [17]. For this, however, there should be compatibility between the bio-crude and fossil stream. As an initial product quality evaluation, the molecular weight of bio-crudes produced in guaiacol, were compared with typical refinery streams (Figure 2). Wood liquefaction bio-crude showed a wide weight distribution, whereas the refinery 
streams are, as expected, cut off molecular weight distributions. If dry wood was used, the molecular weight showed a heavy fraction $(>1 \mathrm{kDa})$ comparable with vacuum residue streams. If water was added, the heaviest components ( $>5 \mathrm{kDa})$ were typically consumed and/or not produced. However, the bio-crude was still significantly heavier than vacuum gas oil and would need a cracking/ depolymerisation (and likely hydrotreatment) step for high-end fuel production. The amount of bio-crude heavier than guaiacol (>150 Da) was determined by GPC and accounted for approximately $55 \mathrm{wt} \%$ of the wood intake, confirming that most of the wood is converted into relatively heavy bio-crude.

Gas production was overall low. The major gaseous products were $\mathrm{CO}$ and $\mathrm{CO}_{2}(\sim 2$ and $1 \mathrm{C} \%$ respectively, table $\mathrm{A} 1$ in Supporting information) with marginal amounts of $\mathrm{CH}_{4}$ and higher hydrocarbons. The $\mathrm{H}_{2}$ production was marginal compared to $\mathrm{CO}_{x}\left(\sim 2 \mathrm{~mol} \%\right.$ of the $\left.\mathrm{CO}_{x}\right)$.

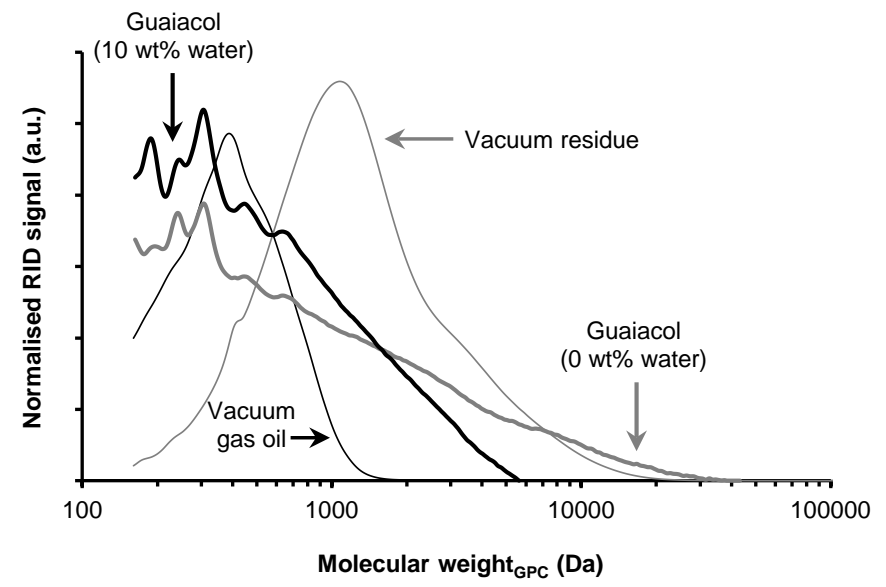

Figure 2. GPC of wood liquefaction using guaiacol as a solvent with and without addition of water plotted together with data of typical refinery streams. Refractive index (arbitrary units) versus polystyrene calibrated molar mass. 


\subsection{Process parameters screening}

Process parameter screening was done for both guaiacol and hexanoic acid as wood liquefaction solvents. As both solvents gave similar trends, we will focus the discussion on the study of guaiacol, which was the most effective liquefaction solvent. The product carbon yield distribution for the process variables (a) wood loading, (b) temperature, (c) reaction time, and (d) water content are shown in Figure 3.
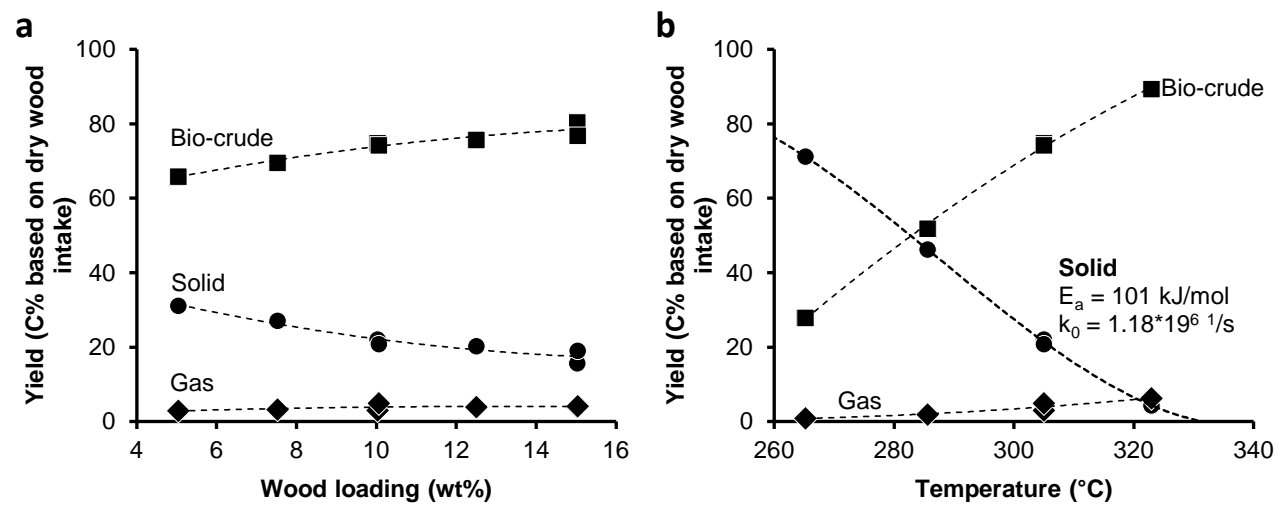

C

\section{d}
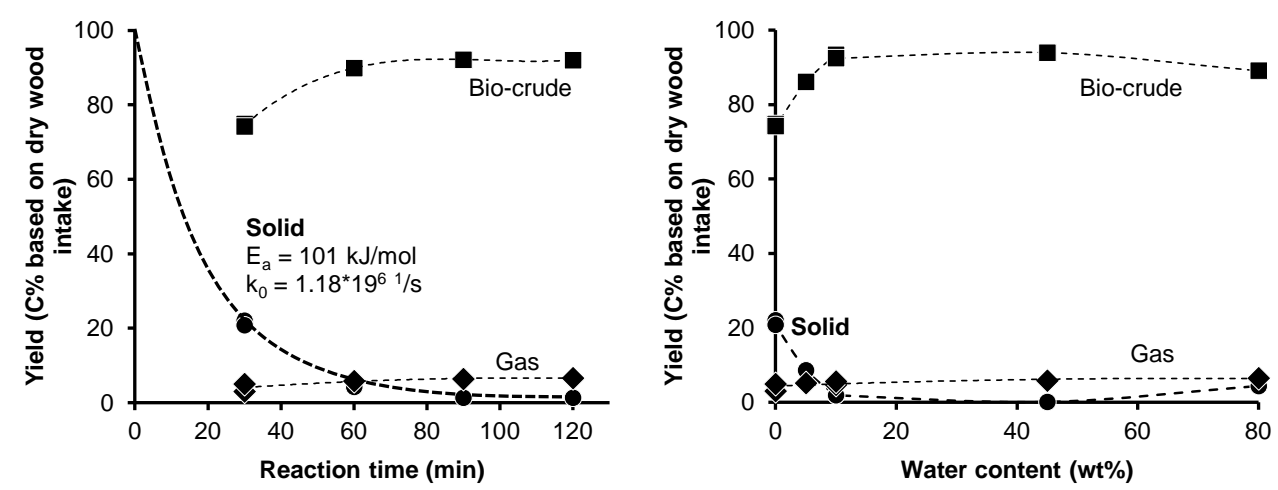

Figure 3. Carbon product yield distribution versus (a) wood loading, (b) temperature (c) reaction time and (d) water content. Standard conditions for an experiment were: $10 \mathrm{wt} \%$ wood, $\sim 30 \mathrm{~min}, \sim 300{ }^{\circ} \mathrm{C}$ and $0 \mathrm{wt} \%$ water, unless specified otherwise by the $\mathrm{X}$-axis. The lines are illustrative except for solid yields versus temperature and reaction time, which are fitted to a first order reaction. 
With increasing wood loading, the bio-crude yield increased as the expense of solid yield. This suggested that a higher concentration of liquefaction intermediates/ products stimulated further liquefaction of biomass polymers. This effect was not observed with wood loading variation using hexanoic acid as a solvent. However, the mixture obtained at high wood loading was very viscous, and much higher loadings would only wet the wood instead of introducing free liquid. Temperature had the strongest effect on liquefaction; as the temperature increased, more solid was converted to bio-crude and permanent gas. The bio-crude yields showed a large increase, the gas yields increase linearly with temperature. The solid residue still had a fibrous appearance similar to the fed wood, which indicated that the solid seems to originate directly from the wood rather than from bio-crude degradation. Longer reaction times resulted in higher bio-crude yields and lower char yields. A small increase of gas yield was observed, which also resulted in a higher pressure (32 bar for $30 \mathrm{~min}$ compared to 45 bar for $120 \mathrm{~min}$, see Table A1 in Supporting information). As already seen with solvent screening, the addition of water had a beneficial effect on the amount of bio-crude produced. Water is normally present in biomass and expensive to remove by drying. Even very high amounts of water seemed to be beneficial at the cost of increasing operating pressure. Although limited, the data allowed a preliminary kinetic analysis of the liquefaction reaction. A first order reaction (Equation 1) was fitted to the temperature and time profiles reported in Figure 3 b-c:

$$
\mathrm{X}=1-\mathrm{e}^{-\mathrm{kt}}
$$

\section{Equation 1}

In which $\mathrm{k}=\mathrm{k}_{0} \mathrm{e}^{-\mathrm{E}_{\mathrm{a}} / \mathrm{RT}}, \mathrm{X}=(1$-solid yield/100) is the conversion of the solid (the solid

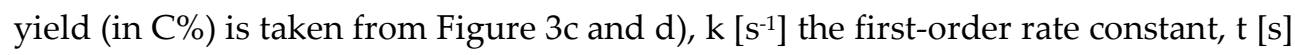
the reaction time, $\mathrm{k}_{0}\left[\mathrm{~s}^{-1}\right]$ a pre-exponential factor, $\mathrm{E}_{\mathrm{a}}\left[\mathrm{kJmol}^{-1}\right]$ the activation energy, $\mathrm{R}\left[\mathrm{kJmol}^{-1} \mathrm{~K}^{-1}\right]=0.008314$ the ideal gas constant, and $\mathrm{T}[\mathrm{K}]$ the temperature. At the same time, the conversion $(\mathrm{X})$ was defined as the decrease in solid residue, assuming that the solid mainly consisted of unconverted feed rather than of solid product 
(char). For the temperature regime examined, this assumption held quite well; however, at higher temperatures a significant amount of char formation is expected. A very good fit was obtained for temperature and reaction time variation (Figure $3 \mathrm{~b}$ and 3c) with an activation energy ( $\left.E_{a}\right)$ of $101 \mathrm{~kJ} \mathrm{~mol}^{-1}$ with a pre-exponential factor (k0) of $1.18^{*} 10^{6} \mathrm{~s}^{-1}$.

\subsection{Process concept}

A possible conceptual process for biomass liquefaction is illustrated in Figure 4. Here biomass is converted in recycled bio-crude, after some tailoring. Light products (gas, water and light organic compounds) and heavy products (solid and very heavy biocrude) are removed, and the large amount of middle-range liquid is used as recycle and product bio-crude. This concept is interesting because it is very simple and could be used for primary conversion at the biomass product site. The product biocrude could then be transported for the final upgrade, for example, by co-refining with fossil streams. In this way, the volumetric energy density is increased significantly and an initial purification step is applied.

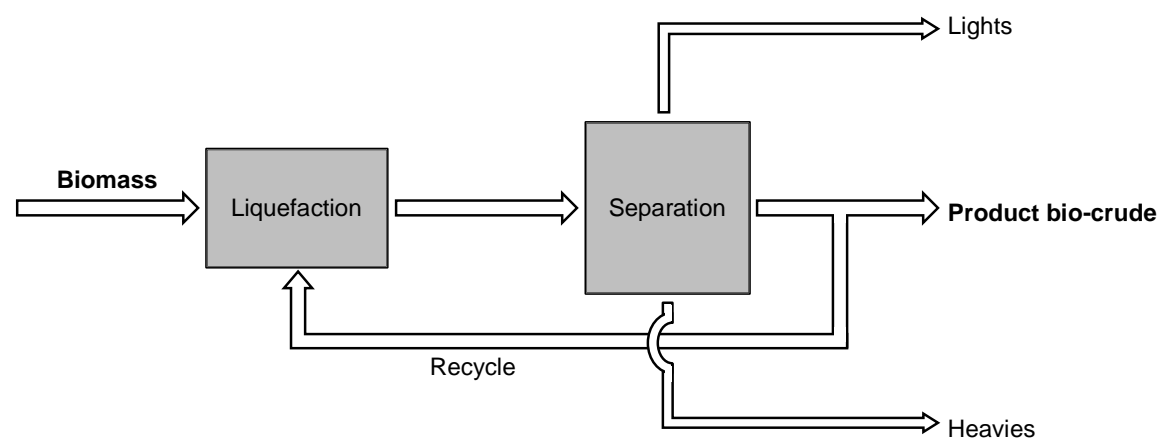

Figure 4. Conceptual process scheme for the liquefaction of biomass for the production of bio-crude 


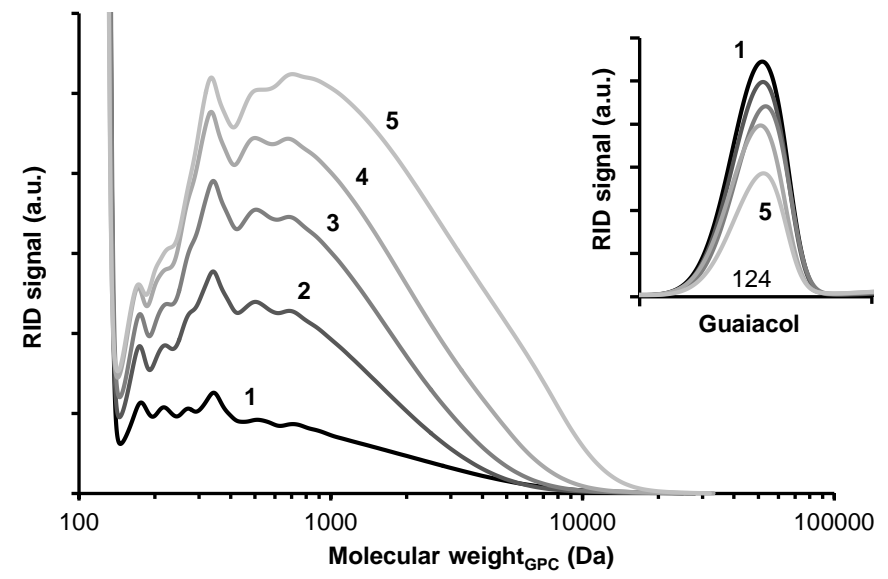

Figure 5. Molecular weight distribution measured via GPC of 5 refill experiments. The insert shows the solvent guaiacol peak.

In an attempt to validate such a concept, a few refill experiments were performed with guaiacol as the start-up solvent and wet wood. As proposed, the light and heavy products were removed between every refill of wood. The light products were removed by releasing the pressure at $200^{\circ} \mathrm{C}$ prior to the final cooling, and the heavy products were removed by filtering the bio-crude through a $1 \mu \mathrm{m}$ filter. With each refill experiment (see also Table A3 in Supporting information), the amount of original guaiacol was reduced to reach approximately $38 \mathrm{wt} \%$ (quantification made with GC-MS) of the initial intake in the last experiment (run 5). As shown in Figure 5 and Table A3 (Supporting information), the overall molecular weight and viscosity of the bio-crude increased with each refill. The overall molecular weight was, after the $5^{\text {th }}$ run, in the same range (heavy end) as vacuum residue and would need significant upgrading for the production of transportation fuels. Interestingly, the effectiveness of the recycle bio-crude remained very high for only low amounts of solids remained after each experiment $(<1 \mathrm{wt} \%$ per refill, see Table A3, Supporting information). It even surpassed the performance of the start-up solvent guaiacol to reduce the amount of solid residue. A similar effect was observed in the process parameter study in which the bio-crude yield increased with increasing wood 
loading (Figure 3a). Elemental analysis (Table A3, Supporting information) shows that significant deoxygenation took place after the fifth run as the oxygen content decreased from approximately $47 \mathrm{wt} \%$ in wood to approximately $32 \mathrm{wt} \%$ in the biocrude. Such an oxygen decrease is consistent with the liberation of water in the lights and $\mathrm{CO}$ and $\mathrm{CO}_{2}$ found in the gas products. It should be specified that the elemental composition of the bio-crude was calculated from that of the liquid product after the contribution of water and guaiacol, which were present in the liquid product, were excluded.

\section{Conclusions}

This chapter reported our search for a cheap biomass liquefaction process that operates at mild pressure, mild temperature, without catalysts and without need for expensive solvent/bio-crude separation. The following could be concluded:

- Polar solvents are more effective than apolar ones as the solvent effectiveness decreases in the order of guaiacol $\approx$ water $>$ hexanoic acid $\gg$ n-undecane $>>$ none. Polar solvents allowed deep liquefaction $(>80 \%)$ at approximately $320^{\circ} \mathrm{C}$ without any catalyst.

- Wet wood led to a higher bio-crude yield and lighter bio-crude than dry wood for all solvents tested. Hence, there is no need to dry the biomass to well before processing. However, addition of water increases the operating pressure significantly.

- For guaiacol, the rate of wood liquefaction appeared to proceed by a first-order reaction in wood with an apparent activation energy of $101 \mathrm{~kJ} \mathrm{~mol}^{-1}$ and a preexponential factor of $1.18^{*} 10^{6} \mathrm{~s}^{-1}$.

- Attempts to recycle the bio-crude through refill experiments showed high biocrude yields with a reduced oxygen content and low solid yields. However, successive refills led to a rapid build-up of heavy products and an increase in 
the viscosity of the liquid. Hence, further reduction or withdrawal of the heavy product prior to recycling seems to be required.

Based on these findings, we propose a simple, small-scale and decentralized liquefaction process that uses the product bio-crude as the liquefaction medium after the removal of the light and heavy products. However, further improvements are still required, particularly to reduce the formation of heavy products or to remove them from the recycled bio-crude via either physical separation or chemical conversion. 


\section{References}

1. Kleinert, M. and Barth, T., Towards a Lignincellulosic Biorefinery: Direct One-Step Conversion of Lignin to Hydrogen-Enriched Biofuel. Energy \& Fuels, 2008. 22(2): p. 13711379.

2. Knežević, D., van Swaaij, W., and Kersten, S., Hydrothermal Conversion Of Biomass. II. Conversion Of Wood, Pyrolysis Oil, And Glucose In Hot Compressed Water. Industrial \& Engineering Chemistry Research, 2009. 49(1): p. 104-112.

3. Czernik, S. and Bridgwater, A.V., Overview of Applications of Biomass Fast Pyrolysis Oil. Energy \& Fuels, 2004. 18(2): p. 590-598.

4. Behrendt, F., Neubauer, Y., Oevermann, M., Wilmes, B., and Zobel, N., Direct Liquefaction of Biomass. Chemical Engineering \& Technology, 2008. 31(5): p. 667-677.

5. Elliott, D.C., Beckman, D., Bridgwater, A.V., Diebold, J.P., Gevert, S.B., and Solantausta, Y., Developments in direct thermochemical liquefaction of biomass: 1983-1990. Energy \& Fuels, 1991. 5(3): p. 399-410.

6. Stevens, J., Young, M., Euhus, D., Coulthard, A., Naae, D., Spilker, K., Hicks, J., Bhattacharya, S., and Spindler, P., Solvent-Enhanced Biomass Liquefaction. 2012, Catchlight Energy LLC.

7. Castellvi Barnes, M., Kersten, S.R.A., Lange, J.-P., and Zhao, W., Process for Conversion of a Cellulosic Material 2013, Shell Internationale Research Maatschappij B.V.

8. Mishra, G. and Saka, S., Kinetic behavior of liquefaction of Japanese beech in subcritical phenol. Bioresource Technology, 2011. 102(23): p. 10946-10950.

9. Maldas, D. and Shiraishi, N., Liquefaction of biomass in the presence of phenol and $\mathrm{H} 2 \mathrm{O}$ using alkalies and salts as the catalyst. Biomass and Bioenergy, 1997. 12(4): p. 273-279.

10. Mun, S.P. and Hassan, E.M., Liquefaction of Lignocellulosic Biomass with Mixtures of Ethanol and Small Amounts of Phenol in the Presence of Methanesulfonic Acid Catalyst. Journal of Industrial and Engineering Chemistry, 2004. 10(5).

11. Mun, S.P. and Hassan, E.M., Liquefaction of Lignocellulosic Biomass with Dioxane/Polar Solvent Mixtures in the Presence of an Acid Catalyst. Journal of Industrial and Engineering Chemistry, 2004. 10(3): p. 473-477. 
12. Beauchet, R., Pinard, L., Kpogbemabou, D., Laduranty, J., Lemee, L., Lemberton, J.L., Bataille, F., Magnoux, P., Ambles, A., and Barbier, J., Hydroliquefaction of green wastes to produce fuels. Bioresource Technology, 2011. 102(10): p. 6200-6207.

13. Rezzoug, S.-A. and Capart, R., Solvolysis and hydrotreatment of wood to provide fuel. Biomass and Bioenergy, 1996. 11(4): p. 343-352.

14. Fan, S.P., Zakaria, S., Chia, C.H., Jamaluddin, F., Nabihah, S., Liew, T.K., and Pua, F.L., Comparative studies of products obtained from solvolysis liquefaction of oil palm empty fruit bunch fibres using different solvents. Bioresour Technol, 2011. 102(3): p. 3521-3526.

15. El-Gayar, M.S. and McAuliffe, C.A., Shellsol as a processing liquid in biomass liquefaction. Energy Sources, 1997. 19(7): p. 665-676.

16. Goudriaan, F. and Peferoen, D.G.R., Liquid fuels from biomass via a hydrothermal process. Chemical Engineering Science, 1990. 45(8): p. 2729-2734.

17. de Miguel Mercader, F., Groeneveld, M.J., Kersten, S.R.A., Way, N.W.J., Schaverien, C.J., and Hogendoorn, J.A., Production of advanced biofuels: Co-processing of upgraded pyrolysis oil in standard refinery units. Applied Catalysis B: Environmental, 2010. 96(1-2): p. 57-66. 


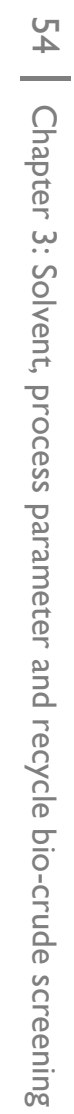




\section{Chapter 4}

\section{Bio-crude characterization based on GPC preparative fractionation}


Analyses of bio-crudes obtained by direct liquefaction are obstructed by the interferences of the liquefaction solvent. We therefore developed a new analytical approach based on a preparative gel permeation chromatography (GPC) fractionation to remove the solvent from the bio-crude and, if convenient, separate the resulting bio-crude into several fractions according to molecular size. The biocrudes investigated were prepared by liquefaction of pine wood using guaiacol and a guaiacol:water mixture as reaction media. These bio-crudes were fractionated and then analysed by several techniques such as ${ }^{13} \mathrm{C}$ - and ${ }^{1} \mathrm{H}-\mathrm{NMR}$, FTIR, Elemental analysis and carbon residue. The results were compared with those of bio-crudes produced via hydrothermal liquefaction (liquefaction in water) and fast pyrolysis. The analyses showed great differences in product distribution, composition and properties of the bio-crude. For instance, the liquefaction bio-crudes appeared to be leaner in sugar-like components and richer in aromatic molecules than pyrolysis biocrude. Moreover, the aromatic content in the liquefaction bio-crudes indicated that carbohydrates react to form this type of compounds. In comparison with pyrolysis, liquefaction yielded bio-crudes leaner in oxygen, but with higher content of heavies (compounds with apparent molecular weight $>1000 \mathrm{Da}$ ). The higher concentration of heavy products resulted in liquefaction bio-crudes with higher coking tendency than pyrolysis bio-crude. A ranking for the severity of the reaction conditions for the liquefaction experiments is proposed: Guaiacol $<$ Guaiacol/water $<$ Water.

\author{
This chapter has been published as: \\ Castellví Barnés, M.; Lange, J.-P.; van Rossum, G. and Kersten, S. R. A.; A new approach for \\ bio-oil characterization based on gel permeation chromatography preparative fractionation. \\ Journal of Analytical and Applied Pyrolysis, 2015, 113: 444-453.
}




\section{Introduction}

Bio-crudes are complex mixtures of organic compounds that cover a wide range of molecular weights, chemical functionalities and properties, thus making their characterization a challenging process. Furthermore, when liquefaction is performed in an organic solvent, the solvent needs to be separated from the biocrude before characterization to avoid its interferences during analyses. In the liquefaction experiments presented below, guaiacol is used as a reaction media because of its efficiency as a liquefaction solvent [1].

The most common fractionation technique used for bio-crudes is solvent extraction. In this procedure, the affinity of the bio-crude components for the different phases is the driving force for fractionation. Thus, the extraction is dictated by the interplay between molecular weight and chemical functionalities, which results in ill-defined fractions. Moreover, multiple steps are involved, which makes the process time consuming ans labour intensive, and leads to poor mass balance closures due to the loss of analytes. Finally, the similarities in chemical structure between guaiacol and a significant fraction of the bio-crudes makes solvent fractionation unsuitable for isolation of these bio-crudes.

Another common technique, distillation, requires high vacuum and relatively high temperatures because of the high boiling point of guaiacol. Performed tests led to insufficient separation of solvent from the bio-crude and poor mass balances. Furthermore, the use of high temperatures can cause thermal degradation of the biocrudes $[2,3]$.

Another fractionation technique was therefore needed, which was capable of isolating the bio-crude without altering its composition. GPC fractionation allows a clean separation of the solvent from the bio-crude based on their differences in molecular size. After isolation, the bio-crude can be characterised and compared with other bio-crudes. In addition, the resulting bio-crude can be separated into several fractions of different molecular size, which can be analysed separately. 
Although GPC has been extensively used for bio-crude characterization, it has been scarcely utilised for its fractionation. Sheu et al. used GPC fractionation to separate pyrolytic tar and its hydrogenated products into fractions with different functionalities [4]. They separated heavy non-volatiles, light non-volatiles + alkanes, phenols and aromatics based on the assumption that similar chemical species have similar molecular sizes. Subsequently, high-resolution gas chromatography/mass spectrometry allowed them to monitor the changes in pyrolytic tar composition during hydrogenation. Andersson et al. used GPC fractionation at analytical scale to separate phenols from the high molecular weight polyaromatics in pyrolysis biocrudes [5]. After fractionation, they analysed the phenolic fraction by multidimensional liquid chromatography (LC-LC). They proved that the GPC and LC-LC system gives reproducible results that are comparable to reference methods. GPC fractionation has also been successfully applied at preparative scale to study the change in composition of coal extracts and solvent refined coal fractions as a function of their molecular size [6-8].

The aim of this chapter is to establish a fractionation and characterization procedure that allows the isolation and characterization of bio-crude, especially those obtained after direct liquefaction in organic solvents. Bio-crudes submitted to the new procedure were prepared by liquefaction of pinewood in guaiacol and in a guaiacol:water (1:1 in weight) mixture at $300^{\circ} \mathrm{C}$ (hereafter, GL stands for liquefaction in guaiacol, and GWL stands for liquefaction in the guaiacol:water mixture). They were then fractionated and analysed by means of various methods such as ${ }^{13} \mathrm{C}$ and ${ }^{1} \mathrm{H}-\mathrm{NMR}$, FTIR, Elemental analysis and carbon residue (MCRT). The results were compared with those obtained for fast pyrolysis bio-crude, hydrothermal liquefaction (liquefaction in water, named WL) bio-crude and organosolv lignin. Fractionation was performed both for bio-crude isolation (removal of the liquefaction solvent) and for bio-crude separation into fractions of different molecular sizes. 


\section{Results}

\section{I. Procedure}

Four bio-crudes were prepared, analysed and compared. Three of them were obtained by liquefaction of pine wood in a $45 \mathrm{ml}$ batch autoclave at $300^{\circ} \mathrm{C}$ and autogenous pressure during 30 minutes. For each of them, a different liquefaction medium was used, namely guaiacol, a guaiacol:water mixture (weight ratio 1:1) and water (which correspond to the well-studied hydrothermal liquefaction, WL). The fourth bio-crude was obtained by fast pyrolysis at the optimal temperature and time for bio-crude production, namely $500^{\circ} \mathrm{C}$ with residence times of 20-25 minutes for the solid particles and below 2 seconds for the pyrolysis vapours that will compose the bio-crude.

The liquefaction and pyrolysis bio-crudes were fractionated into three different fractions based on molecular weight. The two heavier fractions (named middle and heavy fraction) contained bio-crude. The lightest fraction (named light fraction) consisted mainly of guaiacol for both GL and GWL, and, therefore, was not analysed. The lightest fraction of the WL and pyrolysis bio-crudes contained the light bio-crude components and was thus analysed. Care was taken to collect samples that were large enough (at least $0.3 \mathrm{~g}$ ) to allow for detailed analysis (GPC, ${ }^{13} \mathrm{C}-\mathrm{NMR},{ }^{1} \mathrm{H}-\mathrm{NMR}$ and Elemental analysis (EA)). The characterisation results of the two or three fractions were numerically combined to obtain the composition of the whole bio-crude. In addition, each bio-crude was fractionated in only two fractions, one containing mainly the solvent and another containing bio-crude, to subject the bio-crude fraction to MCRT analysis.

\subsection{Product yields and distribution}

The presence and nature of the solvent during thermal treatment proved to have a large effect on products distribution and properties. For instance, GL, GWL and WL 
resulted higher bio-crude yields than fast pyrolysis, even though a higher temperature was used for the latter one (Figure 1). Major differences were also observed in the yield ( $0-27 \mathrm{C} \%)$ and nature of solid residues, being unconverted wood and/or char as revealed by FTIR (Figure D2, Supporting information). Finally, liquefaction led to lower gas yield than pyrolysis did, probably due to a lower reaction temperature.

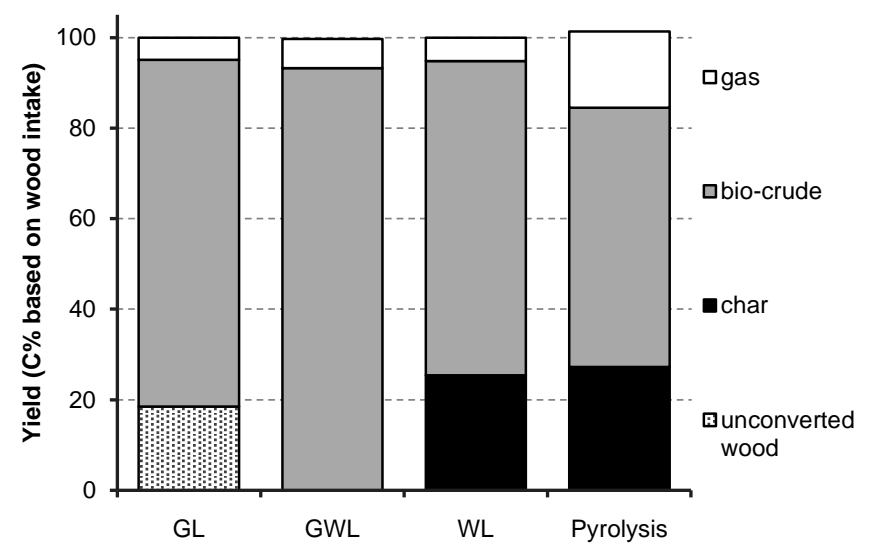

Figure 1. Product distribution for liquefaction and pyrolysis experiments. Liquefaction experiments performed at $300^{\circ} \mathrm{C}$ during 30 minutes with $10 \mathrm{wt} \%$ of wood and $90 \mathrm{wt} \%$ of solvent (guaiacol, guaiacol:water and water). Pyrolysis experiment performed at $500^{\circ} \mathrm{C}$ with residence times of 20-25 minutes for the solid particles and below 2 seconds for the pyrolysis vapours that will compose the bio-crude.

The differences in bio-crude yields are most probably caused by the use of different reaction media in the four experiments. During pyrolysis, formation of liquid intermediates inside the solid particles promote dehydration and cross-linking reactions of the components that are too heavy to vaporise during the reaction conditions. This can lead to repolymerization and, therefore, to char formation [9, 10]. In contrast, the light and heavy molecules formed during liquefaction dissolve in the solvent, which reduces the interactions between them and, presumably, 
reduces their subsequent repolymerization [11]. Within the three liquefaction experiments, which were already reported in the previous chapter, GWL led to the highest liquid yield, with more than $90 \mathrm{C} \%$ of the biomass input being converted to bio-crude. It should be noted that, since the bio-crude yields are calculated by difference, they include the organics dissolved both in the organic and in the aqueous phases that were present in GWL and WL. However, GC-MS analysis of these aqueous phases revealed minor amounts of light oxygenates such as acetic acid and guaiacol. Hence, no further analysis was undertaken as the research majorly focused on the analysis of the middle and heavy fractions of the bio-crudes.

GL led to a high solid yield (19 C\%, Figure 1). However, the solid consisted mainly of unconverted cellulose, as indicated by the carbohydrate fingerprint shown in its FTIR (Figure D2, Supporting information) and its atomic composition represented in Figure 6b. Cellulose is the most recalcitrant component of wood [12] and, therefore, the last component to decompose. Compared to GL, GWL led to a remarkably lower solid yield ( $0.3 \mathrm{C} \%$, Figure 1$)$ that contained both unconverted carbohydrates and aromatic components (Figure D2, Supporting information). Finally, a high solid yield was obtained with WL (25 C\%). The WL solid however, resembled the liquefaction bio-crude and consisted of oxygenated aromatic char (Figure D2, Supporting information). These results are in agreement with those reported by Kumar et al. [13], who observed a change in the composition of the solid residue from wood-type to oxygenated char when the process severity (namely reaction temperature) was increased.

The three liquefaction experiments had similar gas yields of 5-7 C\% whereas the pyrolysis experiment led to a higher gas production of $17 \mathrm{C} \%$ (Figure 1). This is likely a result of the difference in the reaction temperatures, i.e. $300^{\circ} \mathrm{C}$ for the liquefaction and $500^{\circ} \mathrm{C}$ for the pyrolysis. Indeed, Kumar et al [13] also observed an increase in the gas yield with increasing reaction temperature, and Knezevic et. al. [14] observed no variation in the gas yields upon varying reaction medium at a constant temperature (i.e. with the presence (WL) or absence (pyrolysis) of water). 
Based on the product distribution and the composition of the solid, a ranking for the severity of the reaction conditions can be proposed: GL $<$ GWL $<$ WL. Pyrolysis cannot be clearly placed in the ranking since the solid is maintained in the reactor for longer residence times than the bio-crude, which rapidly leaves the reactor as vapour and cools down in the condensers.

\subsection{Characterization of the whole bio-crude}

For characterization of the whole bio-crude without the liquefaction solvent, the results of the two or three bio-crude fractions were numerically combined to obtain the overall bio-crude composition (for more details, see section 2.1). In addition, each bio-crude was fractionated into only two fractions, one containing mainly the solvent and the other containing bio-crude. The bio-crude fraction was subjected to MCRT analysis.

\subsection{GPC and MCRT analyses}

The apparent molecular weight distribution (MwGPC) of the bio-crudes shows the presence of significantly less heavy molecules in pyrolysis bio-crude (up to $2000 \mathrm{Da}$ ) than in the liquefaction bio-crudes (up to $5000 \mathrm{Da}$ for WL and GWL or up to $9000 \mathrm{Da}$ for GL) (Figure 2a). These results are in agreement with the data found in literature [14]. Thus, the liquefaction bio-crudes have a higher concentration of Vacuum Residue (VR, compounds with a MwGPC higher than $1000 \mathrm{Da}$ ) than pyrolysis biocrude does.

MCRT analysis of the isolated bio-crudes revealed the higher coking tendency of the liquefaction bio-crudes compared to the pyrolysis bio-crude. In fact, the cocking tendency of the bio-crudes appeared to be related to their concentration of VR (Figure 2b). Hence, the VR content needs to be minimized to avoid coking during subsequent upgrading to biofuel, e.g. using refinery processes, as proposed by van Rossum et al. [1]. 

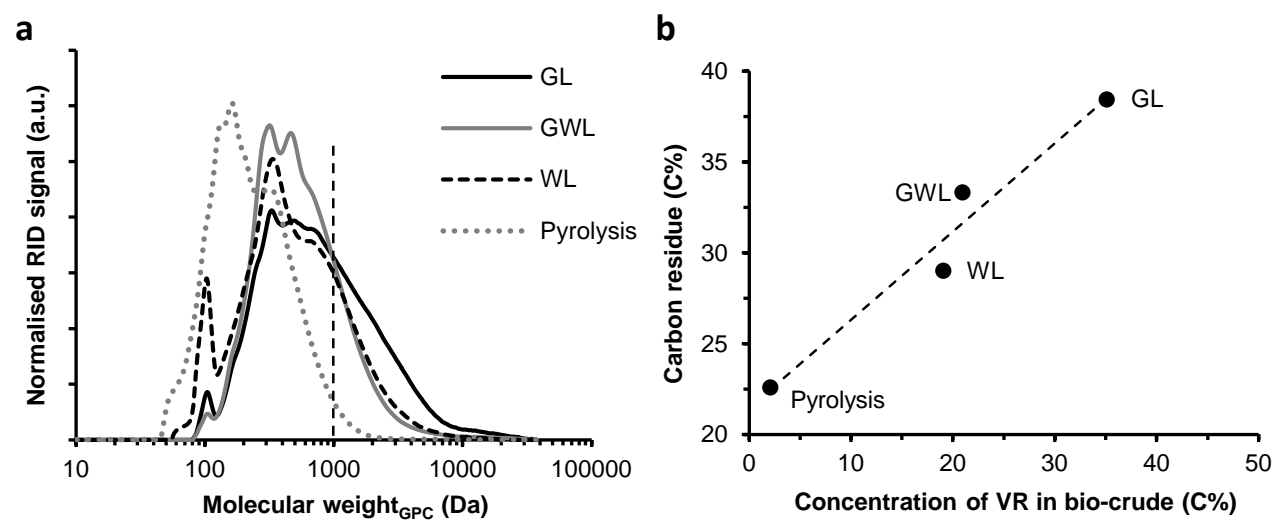

Figure 2. GPC and MCRT results of the isolated bio-crudes. (a) MwGPC distribution of the isolated bio-crudes; the small peak with MwGPC of $113 \mathrm{Da}$ corresponds to a THF decomposition product. (b) MCRT values represented versus the concentration of VR. Liquefaction experiments performed at $300^{\circ} \mathrm{C}$ during 30 minutes with $10 \mathrm{wt} \%$ of wood and $90 \mathrm{wt} \%$ of solvent (guaiacol, guaiacol:water and water). Pyrolysis experiment performed at $500^{\circ} \mathrm{C}$ with residence times of 20-25 minutes for the solid particles and below 2 seconds for the pyrolysis vapours that will compose the bio-crude.

\subsubsection{Elemental analysis}

Elemental analysis of the fractionated bio-crudes revealed a reduction in hydrogen and oxygen content in the bio-crudes compared to the initial biomass. In fact, it showed a lignin-like composition for GL and GWL bio-crudes whereas it suggested a more dehydrated bio-crude for WL and a less dehydrated bio-crude for pyrolysis bio-crude (Figure 3). Between 35 and $45 \%$ of the oxygen present in wood was lost during liquefaction while only $20 \%$ of the oxygen was removed during pyrolysis. Larger deoxygenation during liquefaction in water, compared to pyrolysis, has already been reported in literature [15]. The combined $\mathrm{H}$ and $\mathrm{O}$ losses suggested significant dehydration of the biomass rather than decarboxylation to $\mathrm{CO}_{2}$ (Figure 3). Elimination of oxygen via formation of water is favoured over $\mathrm{CO}_{2}$ formation since dehydration is easier than decarboxylation, which requires a rearrangement of the carbohydrates to form carboxylic acids. Although counter-intuitive, an increase 
of water concentration in the reaction medium seemed to promote the dehydration reactions (compared to GL, GWL has a $10 \%$ and WL a $40 \%$ more dehydration). This can be more clearly observed in the Van Krevelen diagram in Figure D3 (Supporting information) which combines the solids and the bio-crudes numerically. These results are consistent with the extensive dehydration reactions reported in the literature for hydrothermal liquefaction at elevated temperatures and pressures. For instance, degradation of cellulose and hemicellulose was reported to occur through a combination of dehydration and hydrolysis reactions [16].

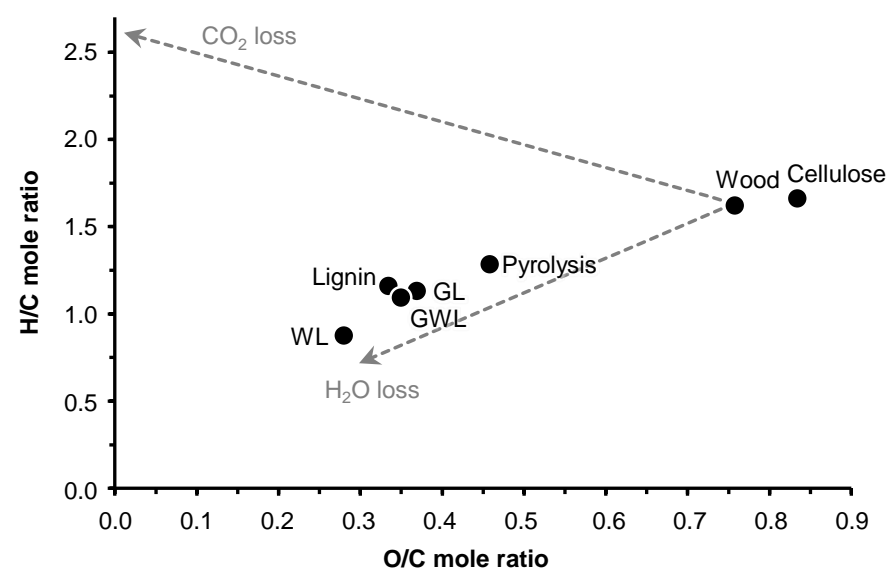

Figure 3. Van Krevelen diagram of isolated bio-crudes and model components. Trend lines for wood dehydration and decarboxylation are also represented. Liquefaction experiments performed at $300^{\circ} \mathrm{C}$ during 30 minutes with $10 \mathrm{wt} \%$ of wood and $90 \mathrm{wt} \%$ of solvent (guaiacol, guaiacol:water and water). Pyrolysis experiment performed at $500^{\circ} \mathrm{C}$ with residence times of 20-25 minutes for the solid particles and below 2 seconds for the pyrolysis vapours that will compose the bio-crude.

\subsection{3. ${ }^{13} \mathrm{C}$ and $\mathbf{H}-\mathrm{NMR}$ analysis}

The ${ }^{13} \mathrm{C}-\mathrm{NMR}$ spectra revealed similar chemical structures for all three liquefaction bio-crudes and organosolv lignin: approximately $60 \mathrm{C} \%$ of unsaturated aromatic 
carbons (105-166 ppm), 30 C\% of saturated alkane-like (1-54 ppm) or carbohydratelike carbons (54-105 ppm) and a minor percentage of unsaturated carbonyl and carboxyl carbons (166-210 ppm). In contrast, the pyrolysis bio-crude contained less aromatic carbons ( $40 \mathrm{C} \%$ vs. $60 \mathrm{C} \%$ ) and more carbohydrate-like carbon (33 C\% vs. $10-22 \mathrm{C} \%$, Figure 4a). The high carbohydrate-like carbon content is in accordance with the high oxygen content found in the pyrolysis bio-crude by elemental analysis (Figure 3). Further details of the ${ }^{13} \mathrm{C}-\mathrm{NMR}$ results are presented in the supporting information (Table D4).

For the three liquefaction experiments, $70-90 \mathrm{C} \%$ of the initial biomass was converted to a bio-crude with a lignin-like composition. However, only a $40 \mathrm{C} \%$ of the initial biomass consisted on lignin. Therefore, some of the carbohydrates present in the wood must had been converted to aromatics that are similar to those contained in lignin. Pyrolysis experiment led to a bio-crude with lower yield and lower aromatic content than the liquefaction bio-crudes. Unfortunately it was not possible to determine if these aromatics have entirely lignin origin or if they are also partly carbohydrate derivatives. In addition, the completely opposite trends followed by the carbohydrate-like and alkane-like carbons suggested that the increase in water concentration in the liquefaction medium promotes the conversion of ether/hydroxyl groups to alkane groups. This shift suggested hydrogen transfer within the bio-crude molecules to form alkane groups either from lignin [17-19] or from carbohydrates [20,21].

${ }^{1} \mathrm{H}-\mathrm{NMR}$ led to the same conclusions as those obtained by ${ }^{13} \mathrm{C}-\mathrm{NMR}$. The liquefaction bio-crudes showed a content of aromatic hydrogen and carbohydrate-like hydrogen which resembled that of organosolv lignin but that contrasts with pyrolysis biocrude (with less aromatic hydrogen and more carbohydrate-like hydrogen than lignin and liquefaction bio-crudes, Figure $4 \mathrm{~b}$ ). The breakdown observed in ${ }^{13} \mathrm{C}$ and ${ }^{1} \mathrm{H}-\mathrm{NMR}$ is not expected to be quantitatively identical because of the varying $\mathrm{H}: \mathrm{C}$ ratio expected for the various types of carbons. Further details of the ${ }^{1} \mathrm{H}-\mathrm{NMR}$ results are presented in the supporting information (Table D5). 

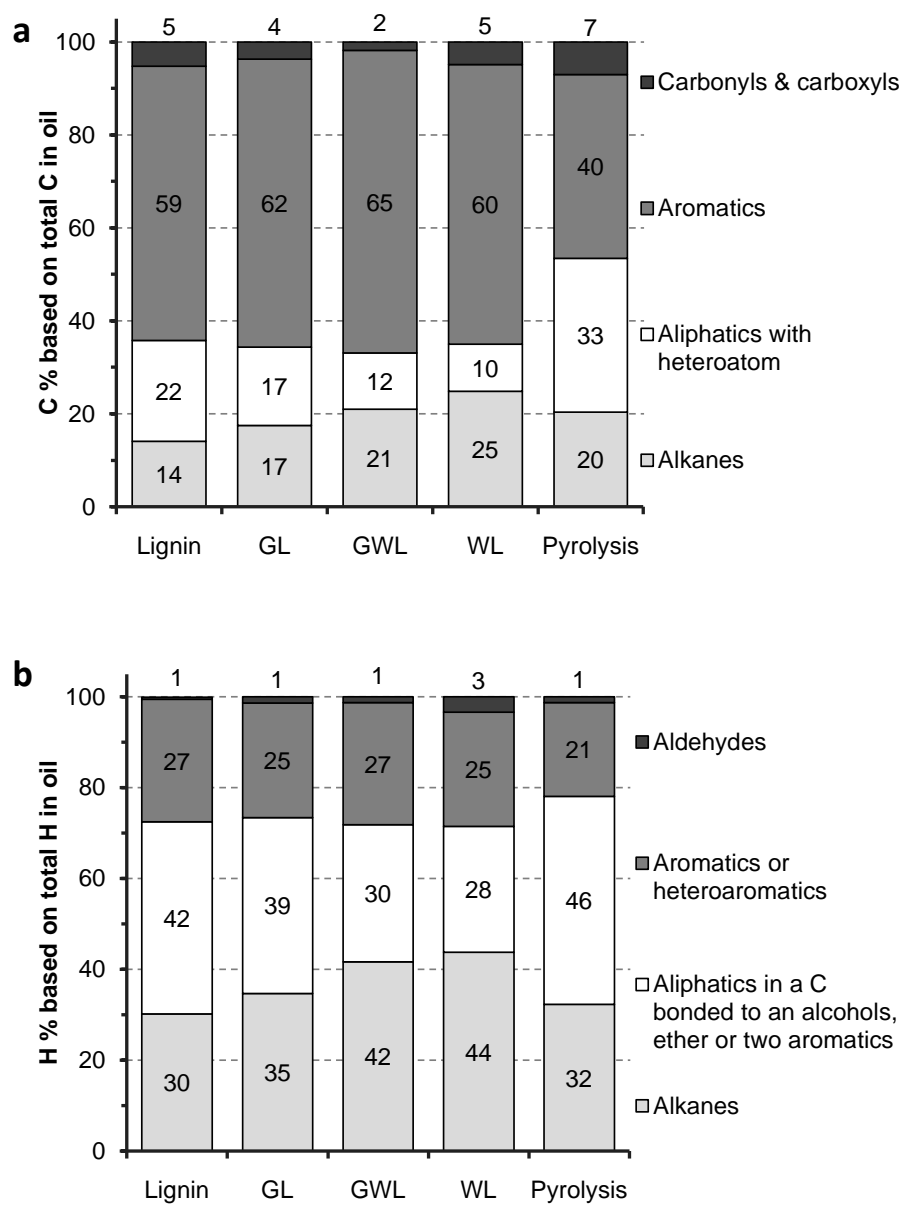

Figure 4. NMR integration results of lignin and isolated bio-crudes. Integration regions for ${ }^{13} \mathrm{C}-\mathrm{NMR}$ (a) and ${ }^{1} \mathrm{H}-\mathrm{NMR}$ (below) assigned according to sections 7.3 and 7.4 , in chapter 2 . Liquefaction experiments performed at $300^{\circ} \mathrm{C}$ during 30 minutes with $10 \mathrm{wt} \%$ of wood and $90 \mathrm{wt} \%$ of solvent (guaiacol, guaiacol:water and water). Pyrolysis experiment performed at $500^{\circ} \mathrm{C}$ with residence times of 2025 minutes for the solid particles and below 2 seconds for the pyrolysis vapours that will compose the bio-crude. 


\subsubsection{FTIR analysis}

The FTIR spectra of the middle fractions of the bio-crudes (Figure 5) point to the same conclusions based on the ${ }^{13} \mathrm{C}$ - and the ${ }^{1} \mathrm{H}-\mathrm{NMR}$ results. The liquefaction biocrudes showed mostly the same vibration bands observed for organosolv lignin: the typical aromatic peaks in the range of $1400-1600 \mathrm{~cm}^{-1}$, an unconjugated carbonyl or carboxyl peak at $1700 \mathrm{~cm}^{-1}$ and the $\mathrm{C}-\mathrm{H}$ stretch from the aromatic molecules over $2900 \mathrm{~cm}^{-1}$. The small concentration of carbohydrates in the liquefaction bio-crudes could be detected through the modest band just over $1000 \mathrm{~cm}^{-1}$ corresponding to the $\mathrm{C}-\mathrm{O}$ stretching vibration and through the $\mathrm{C}-\mathrm{H}$ stretch from aliphatic carbons under $2900 \mathrm{~cm}^{-1}$. The three liquefaction bio-crudes also presented a broad and undefined band at $1200 \mathrm{~cm}^{-1}$, which was best seen for the WL bio-crude. This band has not been clearly assigned to any functionality yet, but it is believed to belong to oxygenated aromatic compounds formed by fused phenols and furans based on evaluation of numerous aromatic components (see supporting information, section C) and literature data [13]. This is in agreement with their higher concentration of aromatic oxygenates compared to pyrolysis bio-crude. In contrast, the pyrolysis bio-crude showed weaker aromatic bands and stronger carbohydrate bands, confirming the lower concentration in lignin-like compounds and the higher concentration of carbohydrate-like components compared to the liquefaction bio-crudes. Besides, the WL bio-crude presented the strongest carbonyl band $\left(1710 \mathrm{~cm}^{-1}\right)$, suggesting a higher concentration of this functional group. However, this result does not completely match with the ${ }^{13} \mathrm{C}-\mathrm{NMR}$ results, in which similar concentrations of carbonyl and carboxyl groups are observed for the four bio-crudes. 


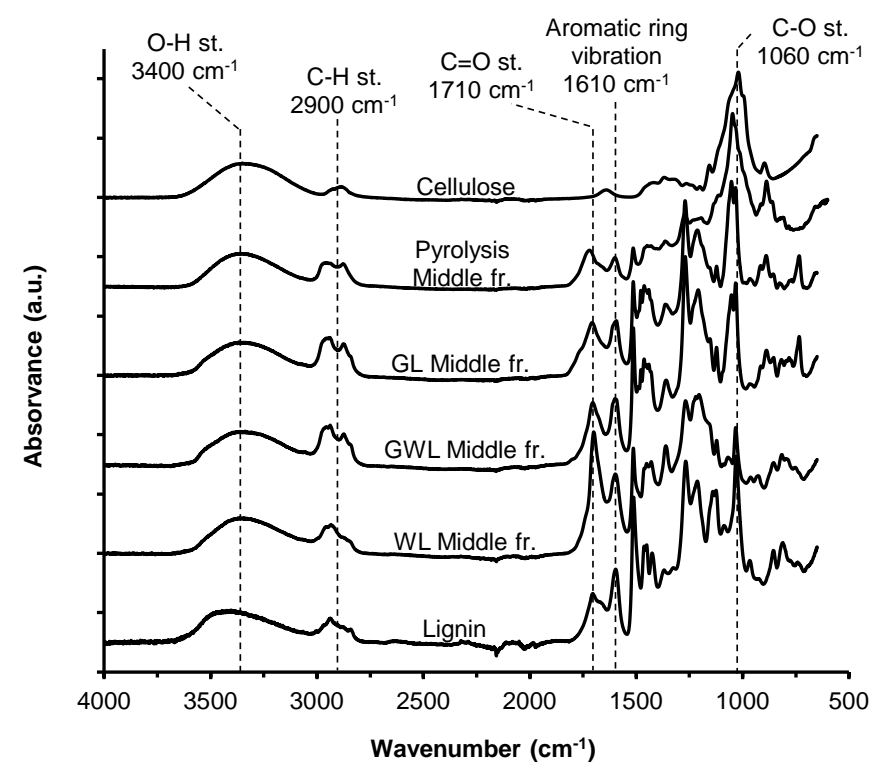

Figure 5. Normalised FTIR spectra of model components and the middle fraction of the liquefaction and pyrolysis bio-crudes. All the FTIR spectra of the fractions of the four bio-crudes can be found in the supporting information (section D). Liquefaction experiments performed at $300^{\circ} \mathrm{C}$ during 30 minutes with $10 \mathrm{wt} \%$ of wood and 90 $w t \%$ of solvent (guaiacol, guaiacol:water and water). Pyrolysis experiment performed at $500^{\circ} \mathrm{C}$ with residence times of 20-25 minutes for the solid particles and below 2 seconds for the pyrolysis vapours that will compose the bio-crude.

\subsection{Characterisation of bio-crude fractions}

As mentioned previously, the liquefaction bio-crudes have a relatively high vacuum residue (VR) content, which causes several problems such as high coking tendency of these bio-crudes. Therefore, a characterization of the VR was performed to determine its composition. To this end, the heavy and the middle fractions (for GL and GWL) and, when convenient, the light fraction (WL and pyrolysis) were 
analysed with GPC, EA, FTIR, ${ }^{1} \mathrm{H}-\mathrm{NMR}$ and ${ }^{13} \mathrm{C}-\mathrm{NMR}$. The quality of the middle and heavy fractions of the GL bio-crude is illustrated in Figure 6. Their corresponding average MwGPC are 360 Da and 1000 Da respectively.
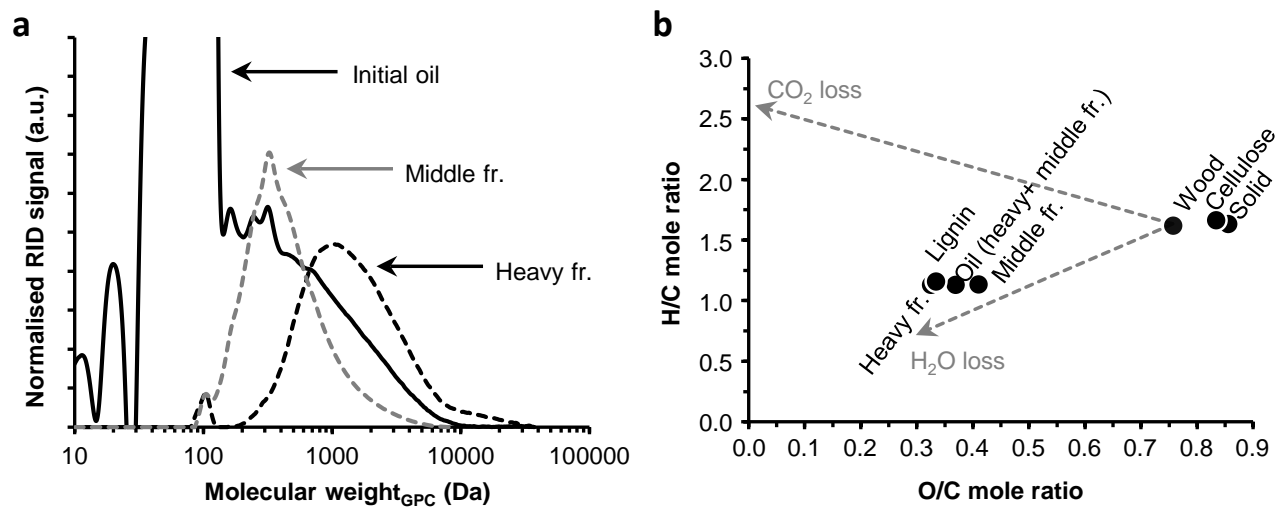

Figure 6. GPC and Van Krevelen diagram of GL bio-crude fractions. (a) Mw distribution and (b) atomic composition of the middle and heavy fraction of bio-crude obtained in guaiacol (GL) (The small peak with MwGPC of 113 Da corresponds to a THF decomposition product. GL performed at $300^{\circ} \mathrm{C}$ during 30 minutes with $10 \mathrm{wt} \%$ of wood and $90 \mathrm{wt} \%$ of guaiacol).

For GL, similar compositions were observed for the middle and the heavy bio-crude fractions. The Elemental composition (Table D3, Supporting information) reveals that the heavy fraction has a lignin-like composition while the middle fraction has a slightly higher oxygen content (Figure 6b). Similarly, the NMR and the FTIR spectra show marginal differences between the two bio-crude fractions (Table D4 and Figure D4, Supporting information).

The middle and heavy fractions of the bio-crude obtained after GWL are also similar in composition and chemical structure, as observed from their elemental composition (Table D3, Supporting information), their ${ }^{13} \mathrm{C}$ - and ${ }^{1} \mathrm{H}-\mathrm{NMR}$ (Table D4 and Table D5, Supporting information), and their FTIR (Figure D6, Supporting information). Both fractions have a composition and structure very similar to lignin. The small differences observed in the elemental composition indicate a slightly 
larger dehydration in the middle fraction than in the heavy fraction. This matches the slightly higher concentration in aromatics and lower in aliphatic components seen in the ${ }^{13} \mathrm{C}-\mathrm{NMR}$ and the ${ }^{1} \mathrm{H}-\mathrm{NMR}$ results.

As for $\mathrm{WL}$, the middle fraction and the heavy fraction had also practically the same composition and structure, as can be observed from their elemental composition (Table D3, Supporting information), FTIR spectra (Figure D8, Supporting information) and ${ }^{13} \mathrm{C}-\mathrm{NMR}$ (Table $\mathrm{D} 4$, in supporting information). The lightest fraction is mainly composed of relatively small molecules that contain remarkably higher $\mathrm{H} / \mathrm{C}$ ratio and lower $\mathrm{O} / \mathrm{C}$ ratio than the two heavier fractions (see elemental composition in Table D3 and GC-MS results in the supporting information, Table D6 and Figure D1a).

The middle and heavy fractions obtained from the pyrolysis bio-crude contain a large concentration of both aromatics and carbohydrates, as measured by FTIR and NMR (Figure D10, Table D4 and Table D5, Supporting information). However, the heavy fraction has a higher concentration of aromatic and alkane-like compounds and a lower concentration of carbohydrates than the middle fraction, namely $48 \mathrm{C} \%$ of aromatics (vs. $40 \mathrm{C} \%$ ) and $25 \mathrm{C} \%$ of alkanes (vs. $18 \mathrm{C} \%$ ). The lightest fraction was mainly composed of small oxygenates (e.g. acetic acid, hydroxyacetone/propanal, furfural and various phenolic compounds) with higher $\mathrm{H} / \mathrm{C}$ and $\mathrm{O} / \mathrm{C}$ ratios than the two heavier fractions (see elemental composition in Table D3 and GC-MS results in Table D6 and Figure D1b in the supporting information).

\section{Discussion}

\section{I. Process severity}

The structure and yield of the solid indicates that the increase in water concentration in the reaction medium increases the reaction severity and therefore the reaction rates during liquefaction. Accordingly, a $19 \mathrm{C} \%$ of unconverted wood is obtained in 
GL, nearly no unconverted wood and no char is produced in the GWL $(0.3 \mathrm{C} \%)$ and a $25 \mathrm{C} \%$ of char is obtained in WL.

The increase in process severity with the increase in water concentration is also apparent in the composition of the liquefaction bio-crudes. The extent of dehydration reactions increases with an increase in water concentration, leading to bio-crudes with a lower oxygen content. Also, the carbohydrates fingerprint in the FTIR and ${ }^{13} \mathrm{C}$-spectra spectra disappear and the typical bands attributed to oxygenated aromatics become more significant.

In conclusion, the severity of the reaction conditions, indicated by the amount of unconverted carbohydrates and oxygen content both in the bio-crude and the solid residue, is: $\mathrm{GL}<\mathrm{GWL}<\mathrm{WL}$.

Determination of severity is more complex for pyrolysis than for liquefaction, because bio-crude and char have different residence times in the hot reaction zone. For instance, residence time is lower than 2 seconds for the bio-crude, but between 20 and 25 minutes for the char. Accordingly, the bio-crude seems to have been produced at a moderate severity, as indicated by its high oxygen and carbohydrate content.

\subsection{Chemistry of liquefaction}

After isolation and fractionation, the chemical composition and structure of the biocrudes and their fractions were analysed. The characterization results revealed that, during wood liquefaction, aromatic and alkane-like carbon is formed. The origin of this type of carbon as well as the possible reaction mechanisms for their formation are discussed below.

NMR and FTIR analyses of the liquefaction bio-crudes reveal an aromatics yield of 40-60 C\% (see section D in Supporting information), which is higher than the aromatics available in the lignin of untreated wood $(25 \mathrm{C} \%)$. This suggests that 
carbohydrates are converted to aromatic products, and supports the dehydration of the initial biomass observed in the Van Krevelen diagrams of the bio-crudes (Figure 3). Unfortunately, the aromatic content in the pyrolysis bio-crude is similar to the aromatic content in the initial biomass, so it cannot be determined whether these aromatics have lignin or carbohydrate origin. The limited formation of $\mathrm{CO}_{2}$ can be attributed to a decarboxylation of organic acids such as levulinic or lactic acid, typically formed during thermal treatment of carbohydrates [22, 23].

The conversion of carbohydrates to aromatic compounds such as furans and phenols during liquefaction and pyrolysis has been largely reported in literature, and numerous reaction mechanisms have been proposed [24-27]. According to most publications, degradation of a carbohydrate polymer, for example cellulose, generally proceeds via a first depolymerisation to form oligomers and monomers (glucose in this case). At those conditions, glucose is relatively unstable and undergoes fast degradation reactions such as dehydration (to form anhydro-sugars), isomerisation (generally to form fructose), or retro-aldol reactions (to form light oxygenated compounds such as glycolaldehyde or dihydroxyacetone). Glucose can also be converted to furanic compounds through dehydration of fructose or anydrosugars [24]. Some publications, however, report formation of furans directly from cellulose, and direct dehydration of cellulose to form furanic polymers [24, 25]. Likewise, formation of benzene or phenolic structures from carbohydrates has also been reported [26-28]. Luijkx et al. [26], for example, observed formation of 1,2,4benzenetriol during hydrothermal liquefaction of 5-(hydroxymethyl)-2-furaldehyde (HMF) and D-fructose. Cheng et al. [28] studied the reaction between furans and olefins over a zeolite catalyst via Diels-Alder condensations, which led to the formation of aromatics with benzene rings. The same type of reaction can be expected between two furanic molecules to form benzene rings. Pastorova et al. [27] characterised the char formed from microcrystalline cellulose and identified several furans with aliphatic side chains and a range of benzene and phenol derivatives. Furthermore, they observed that the aromatic intermediates formed during thermal treatment condensed with other aromatics or small reactive molecules to form char. 
Hence, furanic and phenolic compounds can be formed from thermal treatment of carbohydrates, indicating that the aromatics present in bio-crudes do not necessarily have lignin origin. Unfortunately, none of available analysing techniques could differentiate between furanic and phenolic compounds. This question will be addressed in more depth in the following chapter, which investigates the liquefaction of pure carbohydrates and lignin.

The concentration of alkane-like carbon (aliphatic carbon without oxygen substituents) in the bio-crudes is shown to increase with increasing process severity, which indicates that this type of functionality is formed during liquefaction and pyrolysis of the biomass. Lignin has been reported to partly decompose to aliphatic functionalities. For instance, Ben et al. [17] and Barbier et al. [18]observed their formation during pyrolysis of softwood kraft lignin and during hydrothermal conversion of alkali lignin and model compounds. Formation of alkane-like carbon was also observed by Britt et al. [19] when they performed flash vacuum pyrolysis (FVP) of methoxy-substituted $\beta$-O-4 lignin model compounds. They observed that the main reactions taking place during thermal treatment of lignin were fragmentation of the ether bonds and alkylation of the aromatic rings of the intermediate products. As a result of these reactions, new aliphatic $\mathrm{C}_{\text {aliph- }} \mathrm{C}_{\text {arom }}$ bonds were formed. Alkane-like compounds could also be formed from carbohydrates via hydrogen transfer (intermolecular) or hydrogen shift (intramolecular). For example, Girisuta et al. studied the conversion of glucose to levulinic acid (with three alkanelike carbons) with HMF as a reaction intermediate [20], and Antal et al. observed formation of lactic acid (with one alkane-like carbon) from dihydroxyacetone and glyceraldehyde [21].

Only minor differences in chemical structure are observed between the various fraction of the bio-crudes. Heavy fractions tend to contain slightly more aromatic components than the lighter fractions, which are usually richer in aliphatic compounds. The resemblance between the various fractions of a bio-crude suggests that they are formed via the same type of reactions. 
The next chapter reports on the liquefaction of wood model components (cellulose, hemicellulose and lignin) to deepen the understanding of the chemistry of liquefaction.

\subsection{Bio-crude quality}

Liquefaction appears to remove more oxygen than pyrolysis does (35-45 $\mathrm{wt} \%$ versus $20 \mathrm{wt} \%$ ) and deoxygenation appears to decrease in the order of $\mathrm{WL}>\mathrm{GWL}>\mathrm{GL}>$ Pyrolysis. In all cases, dehydration is the dominant mechanism for oxygen elimination and, consequently, all the bio-crudes have a similar effective hydrogen/carbon ratio of $\mathrm{H} / \mathrm{C}_{\text {eff }}=0.32-0.40$ (Table D2, Supporting information). The equation used to calculate the $\mathrm{H} / \mathrm{C}_{\text {eff }}$ ratio is shown in section 6.3 of chapter 2 . As a result of the oxygen content and the hydrogen/carbon effective ratio, the various liquefaction bio-crudes are estimated to have approximately 50\% higher heating value (HHV) than the wood and approximately $10 \%$ higher than the pyrolysis biocrude (see Table 1). However, the values are still modest compared to typical transportation fuels such as diesel or gasoline. The HHVs were calculated using the Reed's formula [29] (section 6.3 of chapter 2). HHV of diesel and gasoline were obtained from [30].

Table 1. HHV of bio-crudes, model components and fossil fuels.

\begin{tabular}{ll} 
Experiment & HHV $(\mathrm{MJ} / \mathrm{kg})$ \\
GL bio-crude & 25.7 \\
\hline GWL bio-crude & 26.1 \\
\hline WL bio-crude & 27.2 \\
\hline Pyrolysis bio-crude & 23.8 \\
\hline Cellulose & 17.4 \\
\hline Lignin & 27.0 \\
\hline Wood & 18.6 \\
\hline Diesel & 46 \\
\hline Gasoline & $44.8-46.9$ \\
\hline
\end{tabular}

As observed from their composition, the four bio-crudes need to be upgraded before being used as transportation fuels. Upgrading requires deeper deoxygenation as 
well as deeper cracking or depolymerisation. Such upgrading has been applied on a pyrolysis bio-crude, where the bio-crude was subjected to a partial hydrotreatment and, subsequently, to catalytic cracking by co-feeding in an FFC unit [31]. A similar strategy can be adopted for the liquefaction bio-crudes with minor modifications,as they offer the advantage of lower O content but the penalty of higher MwGPC and, consequently, higher coking tendency.

\subsection{Fractionation methodology}

A procedure for bio-crude fractionation with GPC was developed, allowing separation of bio-crude from the liquefaction solvent as well as partition of this biocrude into several fractions based on MwGPc. The use of GPC fractionation enabled an important decrease in the error of the analyses performed (EA, FTIR and MCRT). Furthermore, ${ }^{13} \mathrm{C}-\mathrm{NMR}$ analysis of the bio-crude would have been impossible without fractionation because of the dominant signature of the liquefaction solvent. More details about GPC preparative fractionation are reported in the Supporting information (section B). Nevertheless, further improvements of the fractionation technique should be considered.

In some cases, namely WL and the pyrolysis bio-crudes, a fraction of the bio-crude (MwGPC 300-1000 Da) was lost during fractionation, most likely due to poor dissolution and sticking to vessel walls (see Supporting information, section B). This problem can probably be solved by choosing an adequate eluent that completely dissolves these bio-crudes. Important requirements for this eluent are compatibility with the column packing, having a low boiling point (to be easily removed after fractionation), not promoting specific chemical interaction of the bio-crude with the column packing that could disturb size-based separation and low response in the RI and UV detectors. 
Furthermore, better separation of bio-crude fractions can be obtained by adding more columns into the system or by varying the column packing or the mobile phase.

\section{Conclusions}

Three liquefaction bio-crudes and one fast pyrolysis bio-crude were prepared, analysed and compared. The results led to the following conclusions:

- Characterization of the four bio-crudes showed major differences between the pyrolysis and the liquefaction bio-crudes. Compared to the pyrolysis bio-crude, the three liquefaction bio-crudes showed a lower oxygen content and, correspondingly, a higher heating value. Based on NMR and FTIR, the liquefaction bio-crudes resemble lignin and contain more aromatic (phenolic/furanic) functionalities and less carbohydrate-like functionalities than the pyrolysis bio-crude. However, they also show a much heavier tail, with approximately $1 / 3$ of the bio-crude having an apparent MwGPC beyond $1000 \mathrm{Da}$. As a result, the liquefaction bio-crudes have a much higher coking tendency. Interestingly, the heaviest fraction did not differ significantly from the lightest one in terms of atomic composition and chemical functionalities.

- The yield of aromatic carbon exceeds the lignin content of wood, hence, aromatics have to originate from carbohydrates. Besides, alkane-like carbon is also produced, which can have either or both lignin and carbohydrate origin.

- Dehydration was found to be the main deoxygenation mechanism in the four processes and, within liquefaction bio-crudes, the extent of dehydration increased with increasing the water concentration in the reaction medium.

- Based on the amount of unconverted carbohydrates and the oxygen content both in the bio-crude and the solid residue, a ranking of the severity of the liquefaction reaction conditions, is proposed as Guaiacol < Guaiacol:water < 
Water. This indicates that the severity increases with increasing the concentration of water in the liquefaction medium. As for Pyrolysis, the biocrude indicates a mild severity while the char indicates a high severity, which can be explained by their respectively short and long residence time.

- The quality of the bio-crudes was improved compared to the initial biomass (in terms of oxygen content, $\mathrm{H} / \mathrm{C}$ effective ratio and Higher Heating Value), but it is still quite distant from the commonly used transport fuels. 


\section{References}

1. van Rossum, G., Zhao, W., Castellvi Barnes, M., Lange, J.-P., and Kersten, S.R.A., Liquefaction of Lignocellulosic Biomass: Solvent, Process Parameter, and Recycle Oil Screening. ChemSusChem, 2013. 7(1): p. $253-259$.

2. Ben, H. and Ragauskas, A.J., In Situ NMR Characterization of Pyrolysis Oil during Accelerated Aging. ChemSusChem, 2012. 5(9): p. 1687-1693.

3. Boucher, M.E., Chaala, A., Pakdel, H., and Roy, C., Bio-oils obtained by vacuum pyrolysis of softwood bark as a liquid fuel for gas turbines. Part II: Stability and ageing of bio-oil and its blends with methanol and a pyrolytic aqueous phase. Biomass and Bioenergy, 2000. 19(5): p. 351-361.

4. Sheu, Y.-H.E., Philip, C.V., Anthony, R.G., and Soltes, E.J., Separation of Functionalities in Pyrolytic Tar by Gel Permeation Chromatography-Gas Chromatography. Journal of Chromatographic Science, 1984. 22(11): p. 497-505.

5. Andersson, T., Hyötyläinen, T., and Riekkola, M.-L., Analysis of phenols in pyrolysis oils by gel permeation chromatography and multidimensional liquid chromatography. Journal of Chromatography A, 2000. 896(1-2): p. 343-349.

6. Richards, D.G., Snape, C.E., Bartle, K.D., Gibson, C., Mulligan, M.J., and Taylor, N., Variation of coal extract structure with molecular mass. Fuel, 1983. 62(6): p. 724-731.

7. Wooton, D.L., Coleman, W.M., Taylor, L.T., and Dorn, H.C., Characterization of organic fractions in solvent-refined coal by quantitative n.m.r. spectroscopy. Fuel, 1978. 57(1): p. 17-21.

8. Curtis, C.W., Hathaway, C.D., Guin, J.A., and Tarrer, A.R., Spectroscopic investigations of solvent-refined coal fractions. Fuel, 1980. 59(8): p. 575-583.

9. Kersten, S. and Garcia-Perez, M., Recent developments in fast pyrolysis of ligno-cellulosic materials. Current Opinion in Biotechnology, 2013. 24(3): p. 414-420.

10. Wang, Z., Pecha, B., Westerhof, R.J.M., Kersten, S.R.A., Li, C.-Z., McDonald, A.G., and Garcia-Perez, M., Effect of Cellulose Crystallinity on Solid/Liquid Phase Reactions Responsible for the Formation of Carbonaceous Residues during Pyrolysis. Industrial \& Engineering Chemistry Research, 2014. 53(8): p. 2940-2955.

11. Akhtar, J. and Amin, N.A.S., A review on process conditions for optimum bio-oil yield in hydrothermal liquefaction of biomass. Renewable and Sustainable Energy Reviews, 2011. 15(3): p. 1615-1624. 
12. Mohan, D., Pittman, C.U., and Steele, P.H., Pyrolysis of Wood/Biomass for Bio-oil: A Critical Review. Energy \& Fuels, 2006. 20(3): p. 848-889.

13. Kumar, S., Lange, J.-P., Van Rossum, G., and Kersten, S.R.A., Liquefaction of Lignocellulose: Process Parameter Study To Minimize Heavy Ends. Industrial \& Engineering Chemistry Research, 2014. 53(29): p. 11668-11676.

14. Knezevic, D., Hydrothermal conversion of biomass. 2009: Enschede. p. 151.

15. Elliott, D.C. and Schiefelbein, G.F., Liquid hydrocarbon fuels from biomass. 1989, Pacific Northwest Laboratory.

16. Peterson, A.A., Vogel, F., Lachance, R.P., Fröling, M., Antal, J.M.J., and Tester, J.W., Thermochemical biofuel production in hydrothermal media: A review of sub-and supercritical water technologies. Energy \& Environmental Science, 2008. 1(1): p. 32.

17. Ben, H. and Ragauskas, A.J., NMR Characterization of Pyrolysis Oils from Kraft Lignin. Energy \& Fuels, 2011. 25(5): p. 2322-2332.

18. Barbier, J., Charon, N., Dupassieux, N., Loppinet-Serani, A., Mahé, L., Ponthus, J., Courtiade, M., Ducrozet, A., Quoineaud, A.-A., and Cansell, F., Hydrothermal conversion of lignin compounds. A detailed study of fragmentation and condensation reaction pathways. Biomass and Bioenergy, 2012. 46(0): p. 479-491.

19. Britt, P.F., Buchanan, A.C., Cooney, M.J., and Martineau, D.R., Flash Vacuum Pyrolysis of Methoxy-Substituted Lignin Model Compounds. The Journal of Organic Chemistry, 2000. 65(5): p. 1376-1389.

20. Girisuta, B., Janssen, L.P.B.M., and Heeres, H.J., Green Chemicals: A Kinetic Study on the Conversion of Glucose to Levulinic Acid. Chemical Engineering Research and Design, 2006. 84(5): p. 339-349.

21. Antal Jr, M.J., Mok, W.S.L., and Richards, G.N., Four-carbon model compounds for the reactions of sugars in water at high temperature. Carbohydrate Research, 1990. 199(1): p. 111115.

22. Shafizadeh, F. and Lai, Y.Z., Thermal degradation of 1,6-anhydro-.beta.-D-glucopyranose. The Journal of Organic Chemistry, 1972. 37(2): p. 278-284.

23. Yang, H., Yan, R., Chen, H., Lee, D.H., and Zheng, C., Characteristics of hemicellulose, cellulose and lignin pyrolysis. Fuel, 2007. 86(12-13): p. 1781-1788. 
24. Collard, F.-X. and Blin, J., A review on pyrolysis of biomass constituents: Mechanisms and composition of the products obtained from the conversion of cellulose, hemicelluloses and lignin. Renewable and Sustainable Energy Reviews, 2014. 38(0): p. 594-608.

25. Mettler, M.S., Mushrif, S.H., Paulsen, A.D., Javadekar, A.D., Vlachos, D.G., and Dauenhauer, P.J., Revealing pyrolysis chemistry for biofuels production: Conversion of cellulose to furans and small oxygenates. Energy \& Environmental Science, 2012. 5(1): p. 5414.

26. Luijkx, G.C.A., van Rantwijk, F., and van Bekkum, H., Hydrothermal formation of 1,2,4benzenetriol from 5-hydroxymethyl-2-furaldehyde and d-fructose. Carbohydrate Research, 1993. 242(0): p. 131-139.

27. Pastorova, I., Botto, R.E., Arisz, P.W., and Boon, J.J., Cellulose char structure: a combined analytical Py-GC-MS, FTIR, and NMR study. Carbohydrate Research, 1994. 262(1): p. 2747.

28. Cheng, Y.-T. and Huber, G.W., Production of targeted aromatics by using Diels-Alder classes of reactions with furans and olefins over ZSM-5. Green Chemistry, 2012. 14(11): p. 3114-3125.

29. Domalski, E.S., Jobe, T.L.J., and Milne, T.A., Thermodynamic data for biomass conversion and waste incineration. 1987, American Society of Mechanical Engineers, New York.

30. Laboratory, T.N.P. http://www.kayelaby.npl.co.uk/chemistry/3_11/3_11_4.html.

31. de Miguel Mercader, F., Groeneveld, M.J., Kersten, S.R.A., Way, N.W.J., Schaverien, C.J., and Hogendoorn, J.A., Production of advanced biofuels: Co-processing of upgraded pyrolysis oil in standard refinery units. Applied Catalysis B: Environmental, 2010. 96(1-2): p. 57-66. 


\section{Chapter 5}

Chemistry of the liquefaction of wood and its model components 
Pinewood and various wood model components were liquefied to bio-crude at 300$310^{\circ} \mathrm{C}$ in 1-methylnaphthalene to study the chemistry of the liquefaction process. Cellulose, amylopectin and organosolv lignin were used as model components for the three main constituents of wood, namely cellulose, hemicellulose and lignin. Furthermore, experiments with glucose and wood, were performed for a better understanding of the process. The liquefaction products were analysed by ${ }^{13} \mathrm{C}-\mathrm{NMR}$, FTIR, GPC, GC-MS and Elemental analysis. The results indicated that the produced char originates mainly from the carbohydrates, while the heaviest bio-crude components have mainly lignin origin. Beyond these differences, cellulose, amylopectin and lignin appeared to lead to very similar phenolic-type bio-crude products despite the chemical differences of the three feedstock. In addition, GC-MS results showed that bio-crude from carbohydrates contains both furanic and phenolic aromatics.

This chapter is published as:

Castellví Barnés, M.; de Visser, M. M.; Lange, J.-P.; van Rossum, G. and Kersten, S.R.A.; Chemistry of the Liquefaction of wood and its model components (Submitted to ACS Sustainable Chemistry \& Engineering). 


\section{Introduction:}

Previous work has focused on the direct liquefaction of biomass to obtain a bio-crude that could be blended with refinery streams $[1,2]$. This mixture could then be introduced into existing refinery units for further upgrading to fuel or chemicals. The conversion of biomass into a liquid product was performed via liquefaction in organic medium as this process presented several advantages over other common processes such as pyrolysis. For instance, liquefaction can be operated at milder temperature and higher yields than pyrolysis: high bio-crude yields ( $>90 \mathrm{C} \%$ ) can be obtained for wood liquefaction in the temperature range of $300-350^{\circ} \mathrm{C}[1,3,4]$, while fast pyrolysis of wood performed at the optimal temperature $\left(\sim 500^{\circ} \mathrm{C}\right)$ generally leads to (water-free) bio-crude yields of 50-55 C\%. (N.B.: Higher pyrolysis bio-crude yields of $\sim 65 \mathrm{C} \%{ }^{1}$ have been obtained at lab scale and under optimised conditions (e.g. pyrolysis temperature of $\sim 500^{\circ} \mathrm{C}$ or considerably small particle size) [5-8].

Chapter 4 showed that, in terms of bio-crude composition, liquefaction bio-crude has a lower oxygen content and therefore higher heating value than pyrolysis biocrude. We also observed that liquefaction bio-crude resembles lignin and contains more aromatic/phenolic species and less carbohydrate-like functionalities than pyrolysis oil (chapter 4) [9]. In addition, results revealed that aromatic species have not only lignin, but also carbohydrate origin. Chapter 3 and recent publications also showed that high bio-crude yields can be obtained when using the recycled biocrude or refinery streams as liquefaction medium $[1,2,10]$. Unfortunately, the formation of high concentrations of heavy components remains a challenge in the liquefaction process $[1,2,4,9]$. These heavy components decrease the quality of the bio-crude and hinder its further use or upgrading. For instance, extraction of the heavies is necessary for successful recycling of the light bio-crude as liquefaction medium without pipe blockage due to high viscosity, or coke formation $[1,10]$. In

\footnotetext{
${ }^{1}$ Maximum bio-crude yields reported for fast pyrolysis are around 75-80 wt $\%$. From this 75$80 \mathrm{wt} \%, \sim 65 \mathrm{wt} \%$ is organic liquid and $10-15 \mathrm{wt} \%$ is water. Since the bio-crude and the biomass have approximately the same carbon content ( 50-60 wt \%) we assume a maximum bio-crude yield of $\sim 65 \mathrm{C} \%[5,6]$.
} 
addition, these heavy species have a high propensity to coke during further processing, e.g. in an FCC unit (chapter 4) [9].

Understanding the chemistry of the liquefaction process is essential to know the origin of the various liquefaction products and how they are formed. This becomes specially important when the formation of undesired products, such as heavy biocrude or char, needs to be controlled or prevented. In the current chapter, pinewood and several model components, namely cellulose, amylopectin, lignin and glucose, were liquefied in 1-methylnapthalene. Methylnaphthalene was selected as model component for aromatic solvents. It is also a good representative of aromatic refinery streams such as CGO or LCO, which have been found promising liquefaction media [2]. The liquefaction products were then characterised for a better understanding of the liquefaction reaction mechanisms and to find the origin of the undesired liquefaction products (heavy components in the bio-crude, char and gas) and the aromatic species. Preparative GPC fractionation was used to isolate the bio-crude from the liquefaction solvent before analysis, as performed in chapter 4 .

\section{Results}

\section{I. Procedure}

Several batch experiments were performed in a $45 \mathrm{ml}$ autoclave to establish the optimal conditions for liquefaction of pinewood in 1-methylnaphthalene in terms of bio-crude yield and biomass conversion. Three products were obtained, namely gas, solid (after acetone washing and drying) and bio-crude (see section 2 in chapter 2 for details on liquefaction procedure and product recovery). The optimal liquefaction conditions were found to be $300-310^{\circ} \mathrm{C}, 20$ minutes reaction time, $10 \mathrm{w} \%$ wood and $5 \mathrm{wt} \%$ of water, the balance being methylnaphthalene. Indeed, milder severity, i.e. lower reaction temperature, shorter reaction time, or lower water concentration, resulted in incomplete wood conversion. In contrast, higher severity 
did not seem to increase bio-crude yield significantly. More detailed information on these results can be found in the Supporting information (section E).

Three model components were then selected for comparison with wood, namely crystalline cellulose (Avicel PH-101), amylopectin and organosolv lignin. These are representative of the wood main constituents: cellulose, hemicellulose and lignin. The amylopectin was selected as representative of the hemicellulose because of its amorphous structure, good accessibility and high reactivity for hydrolysis. The organosolv lignin was selected because it is extracted under mild conditions and, thereby, should have undergone minimal degradation. Their liquefaction was performed at the optimal conditions, and the products were characterised with ${ }^{13} \mathrm{C}$ NMR, GPC, FTIR, EA and Micro-GC. In addition, experiments were performed at various reaction times to unravel the reaction sequences. The liquefaction of glucose was also investigated for a better understanding of the chemistry occurring during the process.

\subsection{Product yields}

The product yields obtained after liquefaction of cellulose, amylopectin, glucose and lignin at various reaction times are depicted in Figure 1. The experiments were performed at $300-310^{\circ} \mathrm{C}$ with an intake of $5 \mathrm{wt} \%$ of water, $10 \mathrm{wt} \%$ of biomass, and $85 \mathrm{wt} \%$ of 1 -methylnaphthalene.

After the initial $20 \mathrm{~min}$ of liquefaction, all model components showed a nearly constant bio-crude yield that decreased in the order of lignin > cellulose > amylopectin $>$ glucose. Only cellulose showed a slight decrease in bio-crude yield (and an increase in solid yield) that suggested the presence of some condensation reactions. However, experiments at longer reaction times would be required to confirm this premise. The GPC chromatograms depicted in Figure 1f show that all carbohydrates lead to bio-crudes with considerably less heavy components than the bio-crude from lignin. To be able to quantify the heavy and light species in the bio- 
crude, Vacuum Residue (VR) and Distillate products were defined. VR comprises all the components in the bio-crude that have an apparent $\mathrm{Mw}$ (MwGPC) $>1000 \mathrm{Da}$, and Distillate, the species with MwGPC of 75-1000 Da (see section 6.2 in chapter 2 for further details on the calculation of VR and Distillate yields).

Figure 1f shows that cellulose, amylopectin and glucose yielded bio-crudes with the same $\mathrm{Mw}$ distribution. This result is quite striking since each of these molecules have a different degree of polymerisation and, therefore, a different molecular size. This suggests that the formation of bio-crude proceeds via depolymerisation of the carbohydrate and subsequent repolymerization of carbohydrate or derivative products to form a favoured $\mathrm{Mw}$ distribution. The yield of $\mathrm{VR}(<8 \mathrm{C} \%)$ remained low for the three carbohydrates when the reaction time was increased. Contrarily, the content of distillates in the bio-crude varied significantly depending on the liquefied carbohydrate, and seemed to decrease slightly when the reaction time was increased. The slight decrease in Distillates yield combined with the increase in gas yield suggests that a light fraction of the bio-crude product is not perfectly stable and slowly decomposes to form gas.

In the case of lignin, we show $100 \%$ of bio-crude product at zero reaction time when the other feedstock are shown to deliver $100 \%$ solid instead. Indeed, a blank experiment showed that, at $70^{\circ} \mathrm{C}$, organosolv lignin is already soluble in 1methylnaphthalene. In contrast, carbohydrates are not soluble in 1methylnapthalene or acetone, and therefore, unconverted cellulose, amylopectin or glucose will be found in the solid residue. Upon conversion of the lignin, the amount of bio-crude product barely decreased, and only marginal gas and solid were produced (Figure $1 \mathrm{a}, \mathrm{c}$ and e). However, the content and heaviness of VR in the lignin bio-crude is remarkably high (up to $30 \mathrm{kDa}$ instead of the $3 \mathrm{kDa}$ for carbohydrates, see Figure $1 \mathrm{~b}, \mathrm{~d}$ and $\mathrm{f}$ ). Interestingly, the lignin bio-crude showed the formation of heavy products, heavier than unconverted lignin, after short reaction times and, subsequently, a shift of these heavy products towards lower $\mathrm{Mw}$ upon further reaction (Figure F7, Supporting information). However, the conversion slowed down with time and seemed to stop after 16 hours of reaction. Overall, lignin 
conversion mainly consisted of a partial shift from VR to Distillates, i.e. from 80 to $30 \mathrm{C} \%$ VR yield and from 30 to $65 \mathrm{C} \%$ distillate yield.

A wide range of solid yields was obtained (Figure 1c). Lignin gave the lowest yield (<4 C\%), followed by cellulose (12-26\%), amylopectin (26-29 C\%) and glucose ( 42 $\mathrm{C} \%$ ). Surprisingly, the most reactive carbohydrate (glucose) showed the highest solid yield, while the most recalcitrant one (cellulose) gave the lowest. For glucose and amylopectin, the solid yield seemed to drop within the first 20 minutes to become stable thereafter. Cellulose solid showed a similar behaviour, only with a minor yield increase at long reaction times. Most of the liquefaction experiments yielded a solid residue with an FTIR spectrum that revealed an aromatic char-like structure (discussed later). As exception, the solid obtained at the early stage of cellulose liquefaction (after 10 and 15 minutes) still contained unconverted cellulose.

The gas yields of the three carbohydrates followed similar trends (Figure 1e). In all cases, between 3 and $11 \mathrm{C} \%$ of gas was obtained, with longer reaction times leading to higher gas formation. Lignin liquefaction produced a very low gas yield $(<2 \mathrm{C} \%)$ that remained constant even after 16 hours (960 $\mathrm{min}$ ) of reaction time.

From all model components evaluated here, cellulose behaved most like wood. For both feedstock, a minimum of 20 minutes was required to have complete conversion and, after complete conversion, both yielded around $85 \mathrm{C} \%$ of bio-crude.

To summarise, liquefaction of carbohydrates leads to significant production of char and gas, and a distillate-rich bio-crude (MwGPC of 75-1000 Da). lignin, however, largely dissolves in methylnaphthalene and slowly sees its VR (MwGPC > $1000 \mathrm{Da}$ ) converted to distillate with marginal formation of char and gas. Among the carbohydrates, the recalcitrant cellulose gives the highest bio-crude yield, while the reactive amylopectin and glucose show considerably lower bio-crude yields and higher char yields. 
a

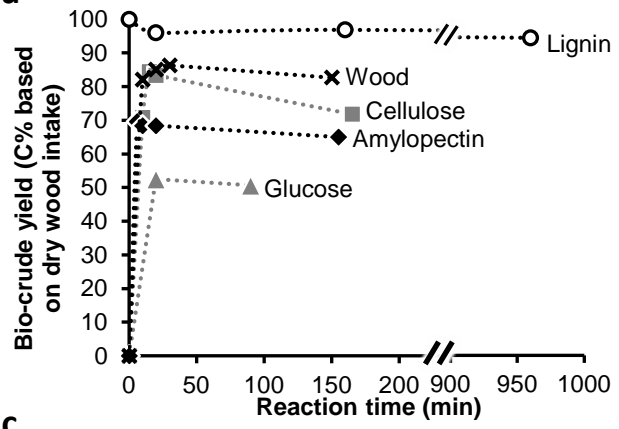

C

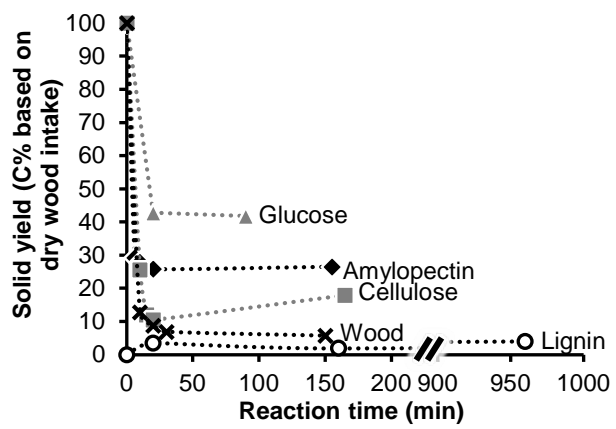

e

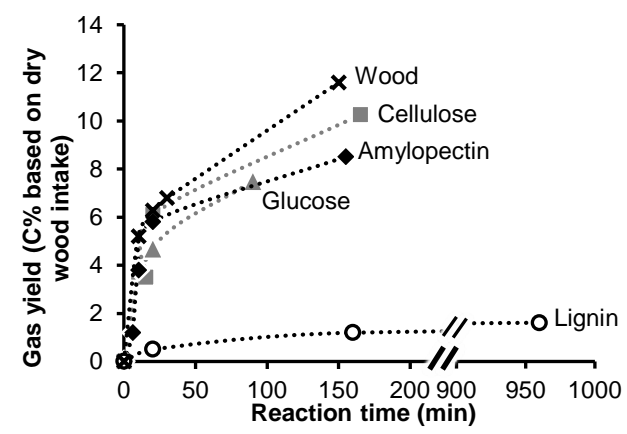

b
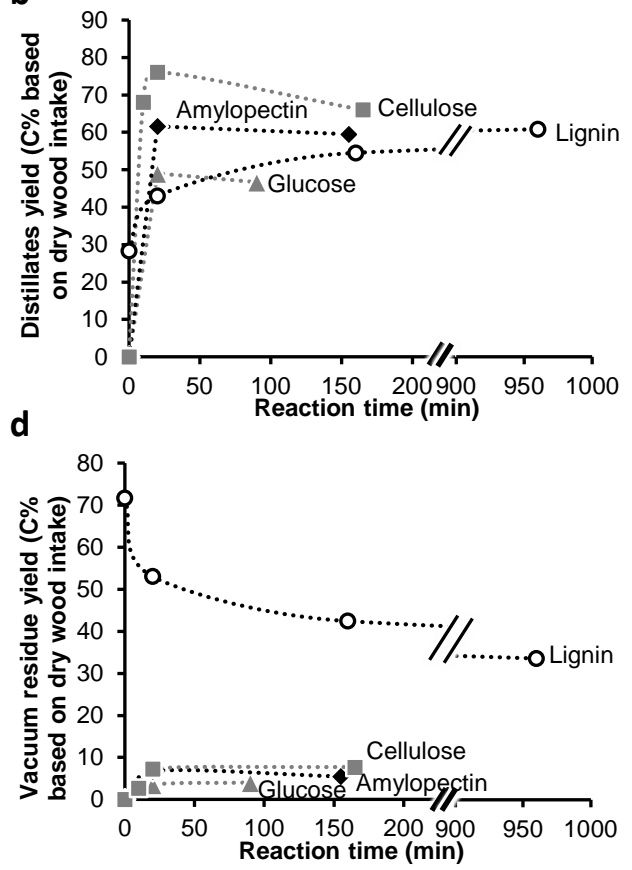

f

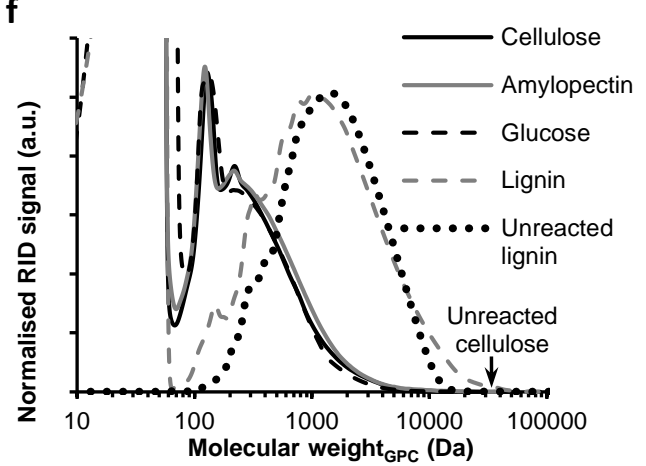

Figure 1. Product yields of the liquefaction experiments (a-e) and molecular weight distribution of the liquefaction bio-crude for the four model components, unreacted lignin and unreacted cellulose ${ }^{2}(f)$. Liquefaction experiments of graphs a-e were performed at 300 $310^{\circ} \mathrm{C}$ with $10 \mathrm{wt} \%$ of biomass, $5 \mathrm{wt} \%$ of water, and $85 \mathrm{wt} \%$ of 1-methylnaphthalene at several reaction times. Liquefaction experiments for graph $\mathrm{f}$ had a reaction time of $20 \mathrm{~min}$.

2 The Mw of the unreacted cellulose was calculated by multiplying the degree of polymerization of Avicel $\mathrm{pH}-101$ reported by Rojas et al. and the Mw of a cellulose monomer [11]. 


\subsection{Product characterization}

The products obtained after liquefaction of lignin, cellulose and amylopectin under reference conditions $\left(300-310^{\circ} \mathrm{C}\right.$ with $10 \mathrm{wt} \%$ of biomass, $5 \mathrm{wt} \%$ of water, $85 \mathrm{wt} \%$ of 1-methylnaphthalene and a reaction time of 20 minutes) were further analysed.

The liquefaction bio-crude was composed of two 'ill defined' phases that were analysed separately: a paste-like phase named bio-tar, which sticks to equipment parts but dissolves in acetone, and a liquid phase named bio-oil. As expected, the tar was richer in heavier components, as seen in the GPC (Figure F4 and Figure F5, Supporting Information). Tar and oil were analysed separately (see following sections) but recombined numerically to define the yields of bio-crude, distillate and VR discussed above. The high concentration of 1-methylnaphthalene in the oil and tar obstructs their characterization. In the previous chapter, preparative GPC fractionation was successfully used to isolate bio-crude from the liquefaction solvent [9]. The same method was used for the current work. Due to their similar MwGPC, some light and volatile molecules were removed with the 1-methylnaphthalene during the fractionation, thus they could not be included in the oil and tar characterization. After preparative GPC fractionation, the solvent-free oil and tar were analysed by FTIR, EA and ${ }^{13} \mathrm{C}-\mathrm{NMR}$. GC-MS analysis of the non-fractionated oils was also performed to identify their light components.

The solid and gas products obtained in all the liquefaction experiments were characterised by FTIR + EA and GC, respectively.

\subsubsection{Oil characterization}

Qualitative GC-MS analysis of the oils revealed a series of aromatic components, mainly 1-methylnaphthalene derivatives and nitrogen-containing aromatics that came as impurities with the solvent (see Table F3 and Figure F10 in Supporting information). Besides, carbohydrate oils presented significant concentrations of light furanic and phenolic components, as well as cyclopentenones. Smaller but 
significant concentrations of small oxygenated molecules, such as acetic acid and hexane-2,5-dione were also observed. In lignin oil, significant concentrations of phenolic components with unsaturated alkyl chains, methoxy, aldehyde or ketone groups were found.

The unmistakable presence of phenolic products in the bio-oil made from pure carbohydrates is worth stressing here. This point will be addressed later in the discussion part of this paper.

Remarkable results were obtained with ${ }^{13} \mathrm{C}-\mathrm{NMR}$ analysis of the oils (Figure 2). Despite having completely different chemical structure, carbohydrates and lignin led to very similar oils in terms of chemical functionality: e.g. approximately $60 \%$ of the carbons are aromatic-like (105-166 ppm) and $\sim 25 \%$ are paraffinic-like (1-54 ppm). In fact, they all resemble unreacted lignin and just differ in the higher content of paraffin-like components (24-27 C\% vs. $14 \mathrm{C} \%$ at 1-54 ppm) and a lower concentration of aliphatic alcohols/ethers (6-12 C\% vs. $22 \mathrm{C} \%$ at $55-105 \mathrm{ppm})$. Thus, carbohydrates, which are mainly composed of aliphatic alcohol carbons, are converted to a lignin-like product with high aromatic and significant paraffinic carbon content.

The lower content of aliphatic ether/alcohol and higher content paraffinic-like components in the lignin oil (vs. unreacted lignin), suggests that the lignin decomposes through the cleavage of ether bonds and the formation of aliphatic species. Lignin presents two major types of ether: primary ethers from the methoxy substituents, and secondary ethers that connect the aromatic units composing the lignin. According to ${ }^{13} \mathrm{C}-\mathrm{NMR}$ results, both primary and secondary ethers are broken during lignin liquefaction (Table F2, Supporting Information).

Elemental analyses confirmed the similarity of all the oils with unreacted lignin (Figure 4). The Van Krevelen diagram in Figure 4 indicates a modest decarboxylation/decarbonylation of these liquids, which agrees with the increase in gas yield and the decrease in liquid yield observed, especially, for the carbohydrates 
(Figure 1). The oils present slightly higher effective $\mathrm{H} / \mathrm{C}$ ratios than the corresponding solid residues (Table F1, Supporting Information).

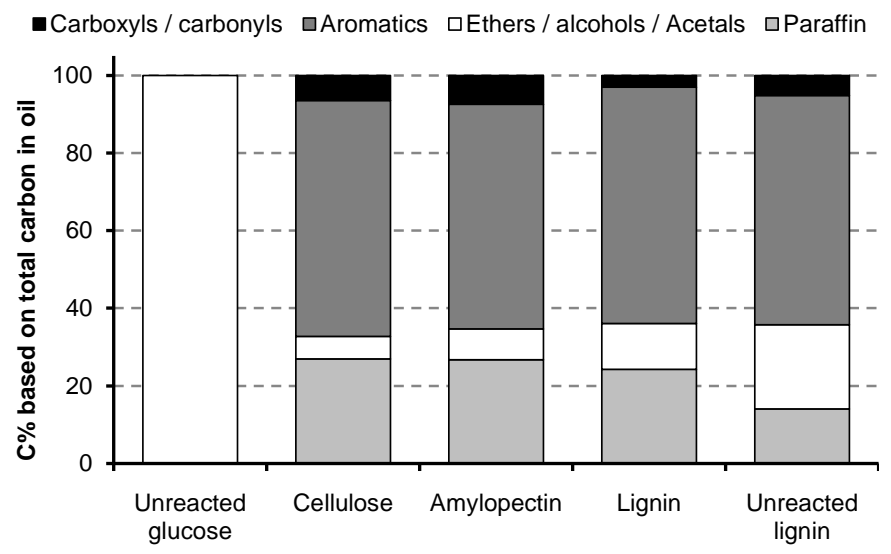

Figure $2 .{ }^{13} \mathrm{C}-\mathrm{NMR}$ integration results of unreacted lignin and solventfree liquefaction oils. Integration regions for ${ }^{13} \mathrm{C}-\mathrm{NMR}$ assigned according section 7.3 in chapter 2 . Liquefaction experiments were performed at $300-310^{\circ} \mathrm{C}$ with a $10 \mathrm{wt} \%$ of biomass, a $5 \mathrm{wt} \%$ of water, an $85 \mathrm{wt} \%$ of 1-methylnaphthalene during $20 \mathrm{~min}$.

\subsubsection{Tar characterization}

Although cellulose, amylopectin and lignin led to very similar char and oil products, larger differences were observed between their tars.

When comparing the oils and tars from cellulose and amylopectin, NMR revealed that tars have a higher content of (partially) unconverted carbohydrates than the oils. This was observed through the higher concentration of oxygenated groups (alcohols, ethers, carbonyls and carboxyls; Table F2, Supporting Information) in the tar. Accordingly, elemental analysis showed higher oxygen content in the bio-tar than in the bio-oils (Figure 4), and FTIR spectra (Figure F3, Supporting Information) of 
bio-tars presented larger sugar C-O stretching band $\left(1000 \mathrm{~cm}^{-1}\right)$ and carbonyl/carboxyl band $\left(1710 \mathrm{~cm}^{-1}\right)$ than the spectra of the bio-oils.

The NMR and elemental analysis results (Figure F2 in Supporting Information, and Figure 4) showed that the lignin tar has nearly the same composition as the unreacted lignin, while the lignin oil presents significantly more paraffin-like components and less alcohol/ether groups. The GPC chromatograms depicted in Figure F4 (Supporting information) also show small differences in Mw distribution between the tar and the unreacted lignin while the bio-oil was considerably lighter with a maximum $\mathrm{Mw}$ of $3 \mathrm{kDa}$ instead of the $10 \mathrm{kDa}$. Finally, the heavier tail of the $\operatorname{tar}(30 \mathrm{kDa}$ instead of $10 \mathrm{kDa}$ for unreacted lignin) suggests some polymerization of the lignin.

\subsubsection{Solid characterization}

FTIR spectroscopy and elemental analysis revealed an aromatic char-like structure for nearly all the solid residues. Characterization results showed that, with sufficiently long reaction times, all the feedstock led to the formation of an aromatic solid (char) that had low hydrogen and oxygen content ( $\mathrm{C}: \mathrm{H}: \mathrm{O}=1: 0.75: 0.25)$, and an FTIR spectrum with broad and undefined bands around $1200 \mathrm{~cm}^{-1}$ that have been assigned to aromatic oxygenates (see Supporting information, section C) [9]. The only exceptions encountered were the solids obtained at the initial stages of cellulose and lignin liquefactions. For reaction times shorter than $20 \mathrm{~min}$, cellulose solid consisted mainly of unconverted cellulose and presented the typical carbohydrate FTIR bands (e.g. C-O stretching band at $1000 \mathrm{~cm}^{-1}$, Figure 3). Similarly, the solid residue from lignin liquefaction at short reaction times presented an FTIR spectrum very similar to that of the unreacted lignin (Figure 3). The FTIR spectra also revealed that, during solid formation, lignin solid loses functionality due to the loss of hydrogen and oxygen. This is reflected in the loss of definition of the FTIR spectra and the decrease of the bands typically attributed to oxygenated functional groups, namely the $\mathrm{O}-\mathrm{H}$ stretching band from the phenol groups $\left(3400 \mathrm{~cm}^{-1}\right)$, the $\mathrm{C}=\mathrm{O}$ 
stretching band typical of carbonyl and carboxyl groups $\left(1710 \mathrm{~cm}^{-1}\right)$, the broad band at $1200 \mathrm{~cm}^{-1}$ attributed to aromatic oxygenates [4, 9] and the C-O stretching band corresponding to aliphatic ether or alcohol groups $\left(1000 \mathrm{~cm}^{-1}\right)$.

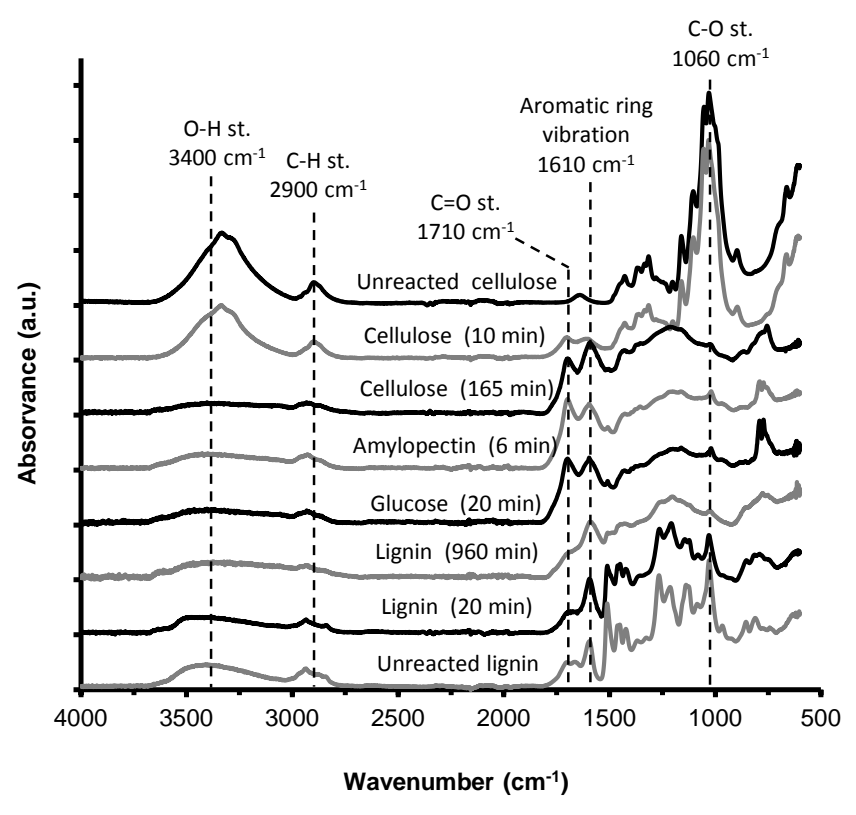

Figure 3. FTIR spectra of model components and solid residues. Liquefaction experiments were performed at $300-310^{\circ} \mathrm{C}$ with a $10 \mathrm{wt} \%$ of biomass, a $5 \mathrm{wt} \%$ of water, an $85 \mathrm{wt} \%$ of 1-methylnaphthalene at the specified reaction times.

The Van Krevelen diagram depicted in Figure 4 confirms that the solid residues from cellulose and amylopectin have the same composition after 20 minutes of liquefaction. The two solids have similar oxygen content than lignin, but they are leaner in hydrogen.

Despite their similar chemical composition, SEM analysis showed completely different morphologies for cellulose and amylopectin solids. While cellulose solid presented a fibrous structure (Figure 5, and Figure F7 in Supporting Information), 
amylopectin solid consisted on large glass-like particles with trapped gas bubbles (Figure 5, and Figure F8 in Supporting Information). Few spheres were observed in the surface of the amylopectin solid particles. Glucose liquefaction (10 $\mathrm{wt} \%$ glucose, $5 \mathrm{wt} \%$ water and $85 \mathrm{wt} \% 1$-methylnaphthalene at $300-310^{\circ} \mathrm{C}$ for $20 \mathrm{~min}$ ) produced a solid similar to the amylopectin solid, but with a rougher surface, and smaller trapped gas bubbles (Figure F9, Supporting Information).

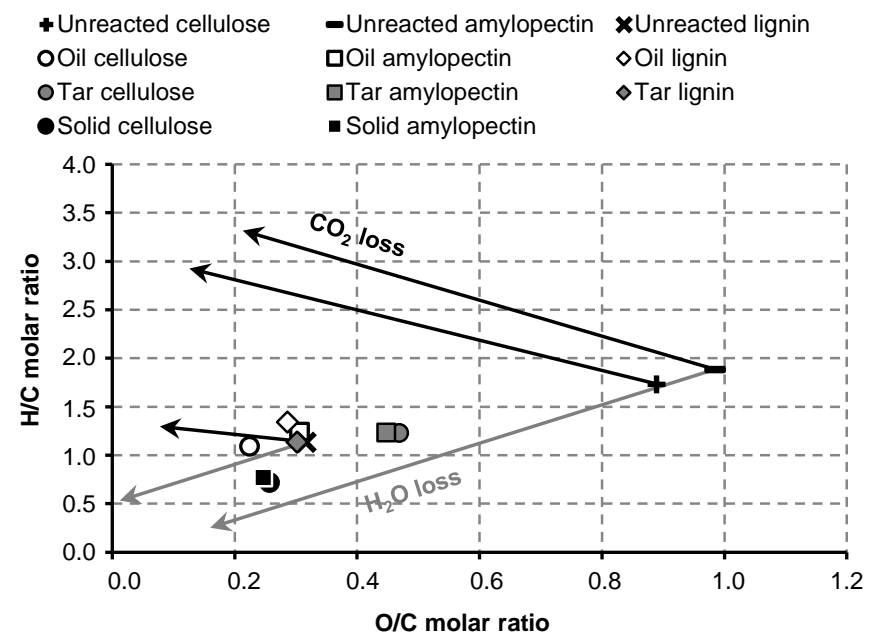

Figure 4. Van Krevelen diagram of model components and the solid and liquid products obtained after their liquefaction. Liquefaction experiments were performed at $300-310^{\circ} \mathrm{C}$ with a $10 \mathrm{wt} \%$ of biomass, a $5 \mathrm{wt} \%$ of water, an $85 \mathrm{wt} \%$ of 1-methylnaphthalene during a reaction time of 20 minutes. 

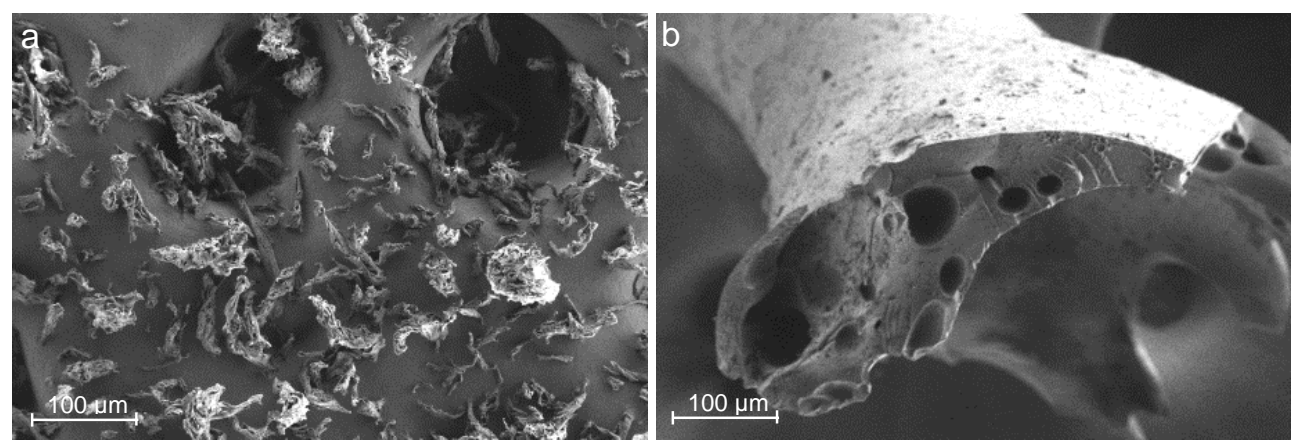

Figure 5. SEM of cellulose (a) and amylopectin (b) chars. Liquefaction performed with 10 $w t \%$ of biomass, $5 \mathrm{wt} \%$ of water and $85 \mathrm{wt} \%$ of 1-methylnaphthalene at $300-310^{\circ} \mathrm{C}$ for 20 min. The background with holes observed on the left image corresponds to the tape used to fix the sample during SEM analysis.

\subsubsection{Gas characterization}

For all liquefaction experiments, $70-80 \mathrm{vol} \%$ of $\mathrm{CO}_{2}$ and $20-25 \mathrm{vol} \%$ of $\mathrm{CO}$ in the gas were obtained. Methane was only formed in marginal amounts. For the carbohydrates, the gas composition did not change with increasing reaction time, but for lignin, a slight increase in $\mathrm{CH}_{4}$ and $\mathrm{H}_{2}$ concentration was observed with increasing time.

Summarising the products characterization, all liquefaction oils resemble unreacted lignin in terms of elemental composition and functional groups. The major differences are found in the compositions and Mw distribution of the tars, which contained unconverted or partially unconverted feedstock. Furthermore, considerably heavier molecules were observed in the lignin bio-crude (VR range) than in the carbohydrates bio-crudes (Distillate range). Under complete conversion, all sugars produced an aromatic char-like solid. This char still shows fibrous structure in the case of cellulose but glassy structure is the case of amylopectin and glucose. 


\subsection{Importance of sugar concentration}

Liquefaction of two different glucose concentrations was performed for a better understanding of the char formation during carbohydrate liquefaction. Results showed that higher glucose concentration leads to higher char formation (Figure 6).

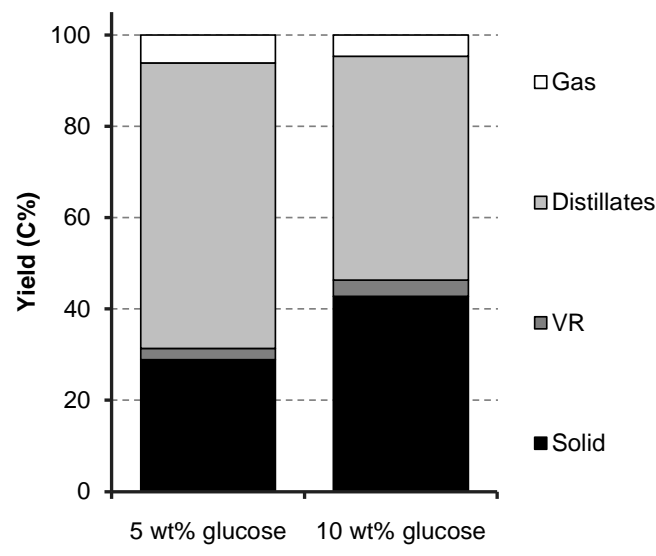

Figure 6. Product yields from glucose experiments. Liquefaction experiments were performed at $300-310^{\circ} \mathrm{C}$ during 20 minutes, and with $5 \mathrm{wt} \%$ of water, $5 / 10 \mathrm{wt} \%$ of glucose and $90 / 85 \mathrm{wt}^{\%} \%$ of 1 methylnaphthalene.

\section{Discussion}

\section{I. Product yields}

The various product fractions show a kinetic behaviour that dominantly corresponds to that of primary reaction products, as observed and discussed extensively earlier [4]. All products are formed shortly after the start of the reaction, without clear induction period, and are subjected to only moderate 'secondary' degradation or formation at long reaction times and deep conversions. Such a description is clearly oversimplified since a few minor deviations from such 
'primary' kinetic behaviour are observed. Firstly, the cellulose bio-crude product undergo minor decomposition with time. Secondly, the gaseous product show significant secondary formation with carbohydrate feedstock. Thirdly, a part of the VR is cracked to distillate in the case of lignin. This behaviour will be discussed in more detail below after discussion of the chemistry of the individual components.

\subsection{Comparison of liquefaction and fast pyrolysis}

As already mentioned in the introduction, liquefaction can be operated at milder conditions $\left(300-350^{\circ} \mathrm{C}\right.$ vs $\sim 500^{\circ} \mathrm{C}$ ) and lead to higher bio-crude yields $(>90 \mathrm{C} \%$ vs 65 $\mathrm{C} \%$ ) than pyrolysis. Furthermore, liquefaction produces bio-crude with lower oxygen content and more aromatic species than pyrolysis. The results presented in the current chapter revealed additional similarities and differences between liquefaction and fast pyrolysis processes.

Lignin appears to be the most recalcitrant wood component for both processes. Due to competitive condensation and fragmentation, lignin is difficult to depolymerise and leads to the formation of heavy species. However, the heavy products find different fates in the two processes. In pyrolysis, these species are too heavy to evaporate and exit the reactor. Hence, they concentrate in the liquid intermediate and eventually overcook to form char [12, 13]. During liquefaction, however, these heavies are dispersed and stabilised by the liquefaction solvent and are, therefore, found as heavy tail in the liquid product [3].

Based on the product distribution and characterization, the char obtained from wood liquefaction has mainly carbohydrate origin. It seems to be formed directly from the condensation of reaction intermediates or dehydration of the initial solid rather than from degradation of the final liquid products. This contrasts also with flash pyrolysis that is capable of cracking all the carbohydrates in volatile components under ideal conditions, e.g. in absence mass transfer limitations [6, 14, 15]. 
In liquefaction, carbohydrates are mostly converted into lignin-like products. This contrasts with pyrolysis, which produces significant concentrations of species containing sugar fragments $[6,9,16,17]$. The formation of aromatics from carbohydrates during liquefaction warrants a more detailed discussion on chemistry, which can be found below.

\subsection{Chemistry of carbohydrates}

Although completely different products yields were obtained from the various carbohydrates (e.g. char yield for cellulose: $12-26 \%$, for amylopectin: $26-29 \mathrm{C} \%$ and for glucose: $\sim 42 \mathrm{C} \%$ ), these products presented very similar characteristics as measured by GC-MS, NMR and FTIR, GPC and/or elemental analysis.

The NMR analysis of the cellulose and amylopectin oils and tars showed mainly aromatics ( 60 and $47 \mathrm{C} \%$ ) and paraffinic-like species ( 26 and $18 \mathrm{C} \%)$. GC-MS analysis further revealed that the aromatic species consists of phenolic as well as furanic species, a discrimination that could not be made by NMR and FTIR. In the following sections, we will try to explain how these species could have been formed from carbohydrates. Char and gas formation will also be discussed.

\subsection{Origin of aromatic species}

The formation of furanic species, either from glucose or directly from carbohydrate polymers has been widely reported in literature [18-21]. However, information on the conversion of carbohydrates to phenolic species is limited. Few reaction mechanisms have been proposed in literature. One of them consists of the hydration of a furanic ring to a dihydroxy species followed by an intra/intermolecular aldol reaction or an electrocyclic rearrangement to form a six-carbon ring that finally dehydrates to form a benzene ring [22]. An alternative mechanism is the formation of phenylic species via the acid- or base-catalysed aldol condensation of small oxygenates created during the liquefaction of carbohydrates [23]. This mechanism is 
in agreement with the results reported by Catallo et al. [24], who liquefied glucose in isotopically labelled water $\left(\mathrm{H}_{2}{ }^{18} \mathrm{O}\right.$ and $\left.\mathrm{D}_{2} \mathrm{O}\right)$ and concluded that the formation of phenolic species needs fragmentation and desaturation of the sugar that is followed by concerted reactions such as Diels-Alder or aldol-condensation reactions. DielsAlder has often been proposed as a mechanism to form benzylic species (e.g. benzofurans) from furans [25]. However, studies made by Vaitheeswaran et al. [26] revealed that the formation of benzofurans from furans over acidic zeolite proceeds via ring-opening mechanisms rather than via Diels-Alder reactions.

Figure 7 depicts 3 possible reaction mechanisms for the conversion of furanics to benzylic species. Although not illustrated in Figure 7, other reaction mechanisms, such as the acid catalysed ring opening reported in [26], are also possible routes for the formation of benzylic structures from furans.

\subsubsection{Origin of paraffinic species:}

The NMR results also revealed an important concentration of paraffin-like $-\mathrm{CH}_{\mathrm{x}}$ fragments. According to GC-MS analyses (Table F3, Supporting Information), these paraffinic carbons can be found as furanic or benzylic substituents, as cyclopentenones or as small aliphatic molecules such as acetic, levulinic or lactic acids $[27,28]$. However, regardless of the final structure, the conversion of carbohydrate-like carbon to a paraffinic-like carbon requires a net oxygen-hydrogen transfer/shift. This most easily proceeds via a combination of deoxygenation and $\mathrm{H}$ shift. 

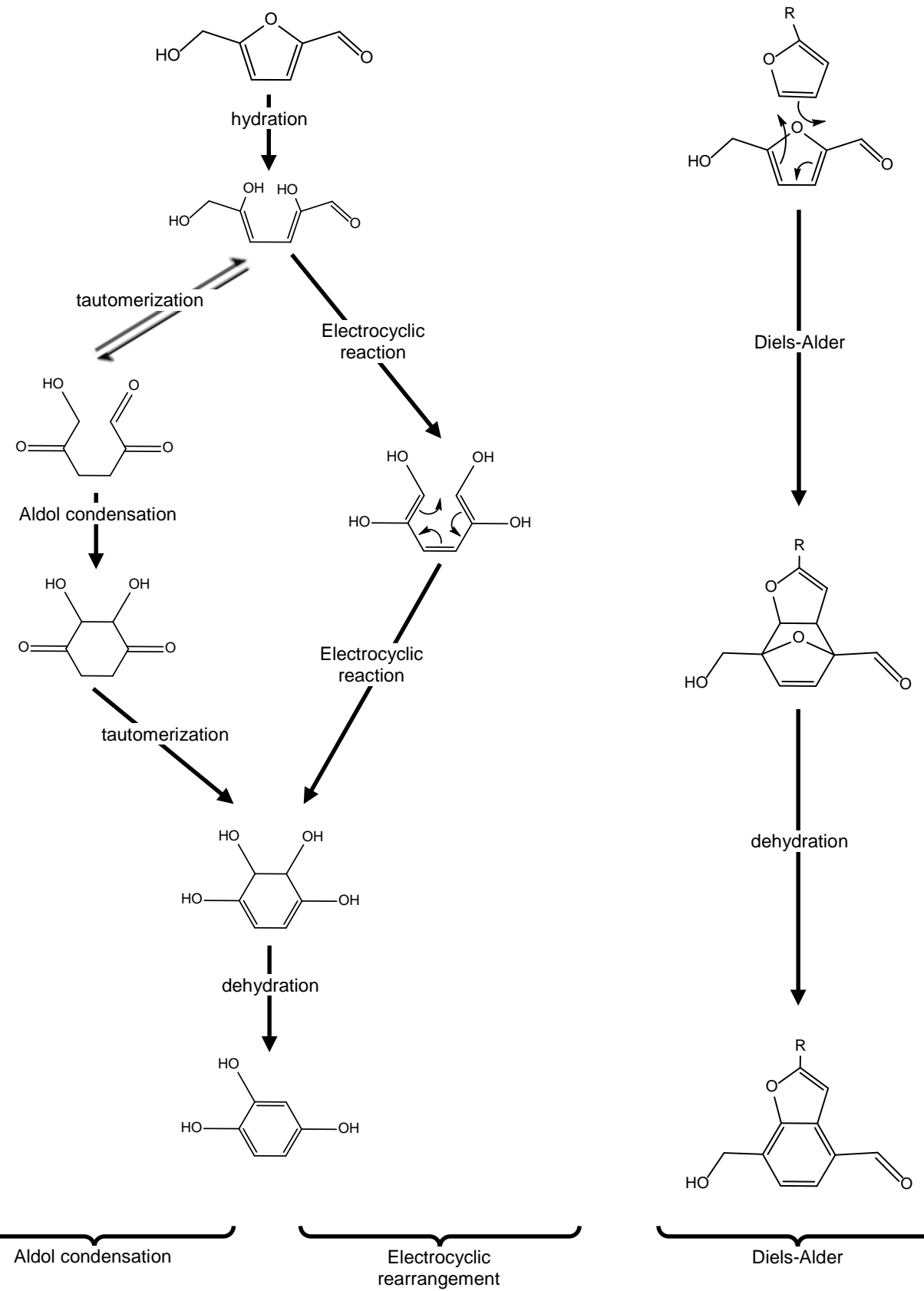

Figure 7. Example of possible reaction routes for the conversion of furanics to phenolic species. 

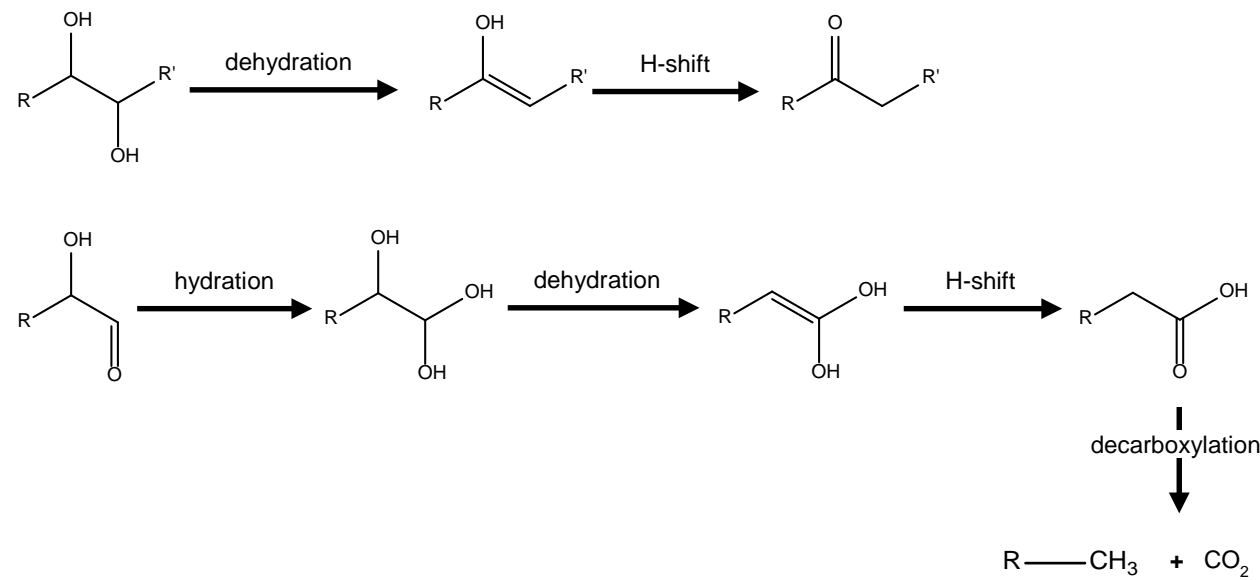

Figure 8. Examples of possible combination of deoxygenation and H-shift for the conversion of carbohydrate-like carbon to paraffinic-like carbon.

Figure 8 shows possible reaction routes for the formation of paraffinic carbon from carbohydrate-like carbon via H-shift and/or decarboxylation. In the first scheme, two sugar-like carbons (with two hydroxy groups) are converted into one carbonylic and one paraffinic carbon via dehydration and subsequent intramolecular H-shift. In the second scheme, two sugar like carbons (with one alcohol and one aldehyde) react through an $-\mathrm{OH}$ shift (via aldehyde hydration + dehydration), followed by a hydrogen shift and finally decarboxylation to yield one paraffinic carbon and one $\mathrm{CO}_{2}$ molecule.

These schemes agree with the results depicted in the Van Krevelen diagram of Figure 4. According to the $\mathrm{O} / \mathrm{C}$ and $\mathrm{H} / \mathrm{C}$ ratios of the cellulose and amylopectin oils and tars, most of the oxygen and hydrogen loss is caused by dehydration. Yet, the data points of the oils and tars fall above the cellulose and amylopectin dehydration lines, indicating that reaction mechanisms involving hydrogen addition or oxygen removal (as $\mathrm{CO}_{2}$ or $\mathrm{CO}$ ) are also occurring. 


\subsubsection{Origin of char}

According to the results presented above, char resembles a 'primary' product with a mostly carbohydrate origin. Thus, char is not significantly formed from the final liquid product, but from early reaction intermediates such as depolymerised sugars or their derivatives. It is highly carbonaceous ( $70 \mathrm{wt} \%$ of $\mathrm{C})$ and lean in oxygen $(\sim 25$ ${ }^{w} \mathrm{t} \%$ of $\mathrm{O}$ ), as it could be observed from the EA results (Table F1, Supporting Information). It could also be suspected from the undefined and broad bands present in the FTIR spectra of all char solids (see section 2.3.3 and Figure 3). Even though the chars from cellulose, amylopectin and glucose have a very similar chemical composition (EA and FTIR), they present different morphologies. While cellulose char has a fibrous structure, amylopectin and glucose chars consist of large and compact glass-like particles with trapped gas bubbles and, eventually, a few small globular particles on the surface (Figure F7, Figure F8 and Figure F9; Supporting information).

According to these results, the low char yields obtained during cellulose liquefaction would form as minor parallel reaction to the oil formation, through dehydration, cross-linking and condensation reactions of poorly depolymerised cellulose, thus preserving cellulose's fibrous structure. On the contrary, amylopectin and glucose, which produced larger amount of char, either undergo more complete depolymerisation or even start with fully depolymerised sugar. Abundant and reactive sugar and/or their derivatives would undergo dehydration, cross-linking and condensation reactions to form heavy components that are poorly soluble in the reaction medium and, eventually, solidify to glassy char. The higher the concentration of reactive sugars, the higher the probability of condensation to char, as observed in section 2.4 .

Wang et al. [29], also observed the formation of fibrous and glassy char during the pyrolysis of cellulose. The fibrous char was observed when pyrolysing crystalline cellulose while the glassy char was obtained from ball-milled (amorphous) cellulose at $400^{\circ} \mathrm{C}$. Interestingly, the char morphologies observed here deviate from those 
obtained upon acid-hydrolysis of carbohydrates and/or their derivatives [30-35]. These humins consisted indeed of aggregates of globular particles. Their formation likely requires a different condensation and precipitation mechanism, possibly via the desolubilisation of liquid components into micelles and their subsequent condensation within the micelles. Few humin particles can be observed on the surface of the amylopectin glassy char (Figure F8, Supporting information).

\subsubsection{Origin of gas}

Gas behaves both as a 'primary' and a 'secondary' product, and has mainly a carbohydrate origin. Lignin liquefaction gives considerably less gas than the sugars. For all the liquefaction experiments, gas is mainly composed of $\mathrm{CO}_{2}$ and some $\mathrm{CO}$, and is linked to the $\mathrm{H}$-shift and deoxygenation of the liquid product. In the initial stage of the liquefaction, $\mathrm{CO}_{2} / \mathrm{CO}$ formation is a result of decarboxylation/ decarbonylation of acids or aldehydes present in sugar-like molecules. In later stages, decarboxylation/ decarbonylation of aromatic species in the liquid product, such as furfural, occurs. A possible route for $\mathrm{CO}_{2}$ formation is illustrated in Figure 8.

\subsection{Chemistry of lignin}

The yields and characterization of the lignin products revealed some information about the reaction mechanisms occurring during its liquefaction. According to the product distribution, nearly constant bio-crude, solid and gas yields were obtained. However, the GPC analysis reveals an initial widening of the Mw distribution, i.e. simultaneous formation of lighter and heavier components, that is followed by a narrowing and shift of the whole Mw distribution towards lower Mw (Figure 9). The characterization results showed that the tar was very similar to the unreacted lignin, while the oil contained less alcohol/ether groups, and more paraffin-like components and aromatic groups. 


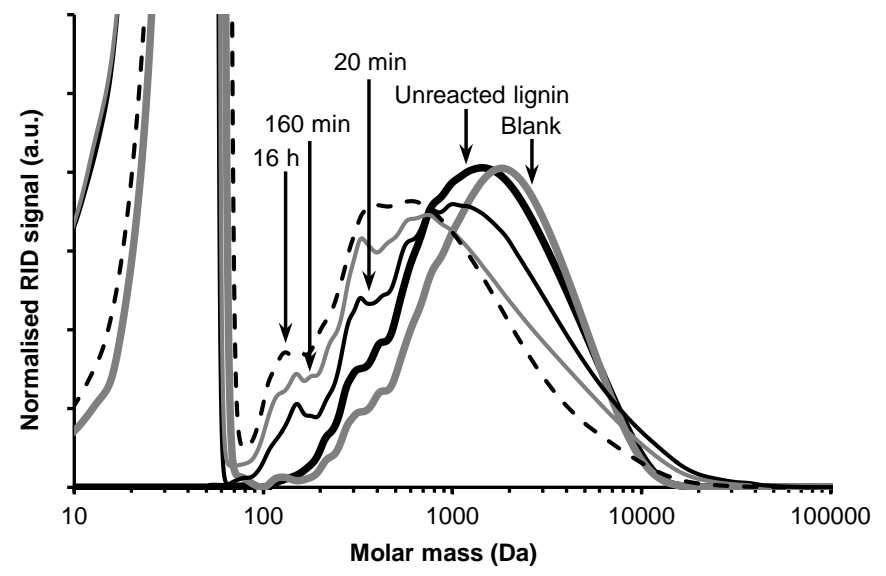

Figure 9. Molecular weight distribution of the blank and the lignin bio-crudes (oil + tar) obtained at various reaction times. Blank experiment was performed at $70^{\circ} \mathrm{C}$ during 20 minutes with $10 \mathrm{wt} \%$ of organosolv lignin, $5 \mathrm{wt} \%$ of water and $85 \mathrm{wt} \%$ of 1 methylnaphthalene. Liquefaction experiments were performed at 300$310^{\circ} \mathrm{C}$ with $10 \mathrm{wt} \%$ of organosolv lignin, $5 \mathrm{wt} \%$ of water and $85 \mathrm{wt} \%$ of 1-methylnaphthalene at the reaction times of $20 \mathrm{~min}, 160 \mathrm{~min}$ and 16 hours.

All these results indicate that lignin liquefaction takes place by a combination of decomposition and condensation reactions. The decomposition likely occurs via the cleavage of the ether bonds that link the lignin units or monomers, which is supported by the lower concentration of this type of group in the bio-oil compared to the unreacted lignin (NMR results, section 2.3).

The cleavage of these ether bonds can proceed via hydrolysis to form two alcohol groups, or via O-elimination to form a $\mathrm{C}=\mathrm{C}$ double bond that is stabilised by conjugation with the aromatic ring.

The condensation reactions could likely proceed by C-C coupling of lignin chain through the alkylation of a benzylic-like carbon to a neighbouring aromatic rings (see NMR results in section 2.3.1). When proceeding intramolecularly, the 
condensation does not change the $\mathrm{Mw}$ of the product but makes it more recalcitrant against further cleavage since it replaces a labile $\mathrm{C}-\mathrm{O}-\mathrm{C}$ bridge by a stronger $\mathrm{C}-\mathrm{C}$ bond. However, intermolecular condensation increases both the $\mathrm{Mw}$ and the recalcitrance of the chain. Simultaneous decomposition and condensation have been reported in literature, and several reaction mechanisms have been proposed, including free-radical reactions, molecular rearrangements and concerted eliminations [36-38].

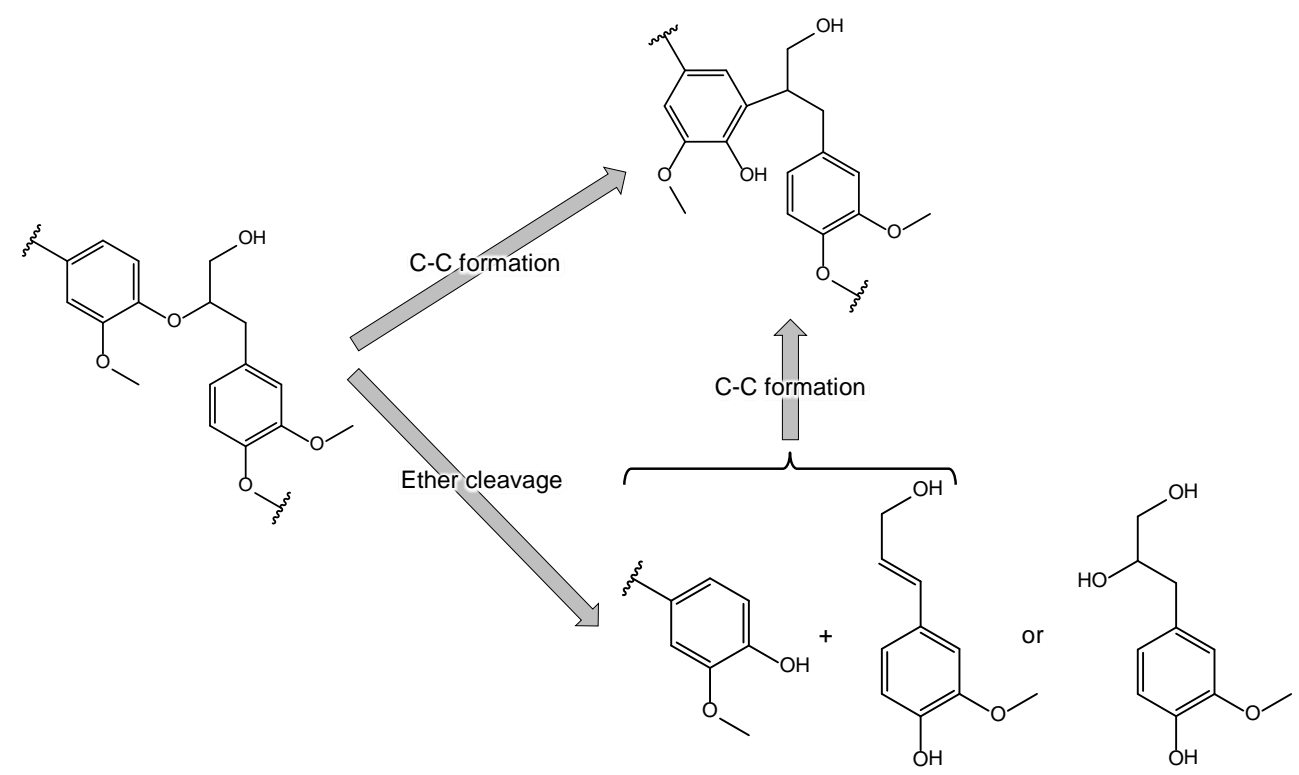

Figure 10. Example of possible intramolecular recondensation and depolymerisation reactions occurring during lignin liquefaction. Intermolecular C-C formation is equally likely than the intramolecular reaction illustrated here.

An example of possible elementary steps for lignin decomposition and condensation is shown in Figure 10. It is worth mentioning that the reaction products represented there might not be stable and react further under liquefaction conditions. It should also be noted that, even though Figure 10 depicts only intramolecular condensation reactions, intermolecular condensation also occurs and is the main cause of the $\mathrm{Mw}$ increase of the liquefaction bio-crude. 


\subsection{Reaction scheme for carbohydrates and lignin}

All the results discussed above can now be compiled into a simplified reaction scheme (Figure 11), a part of which resembles the scheme proposed and discussed by Kumar et al. [4], but is now complemented to account for the behaviour of glucose and amylopectin and describe the specific behaviour of lignin.

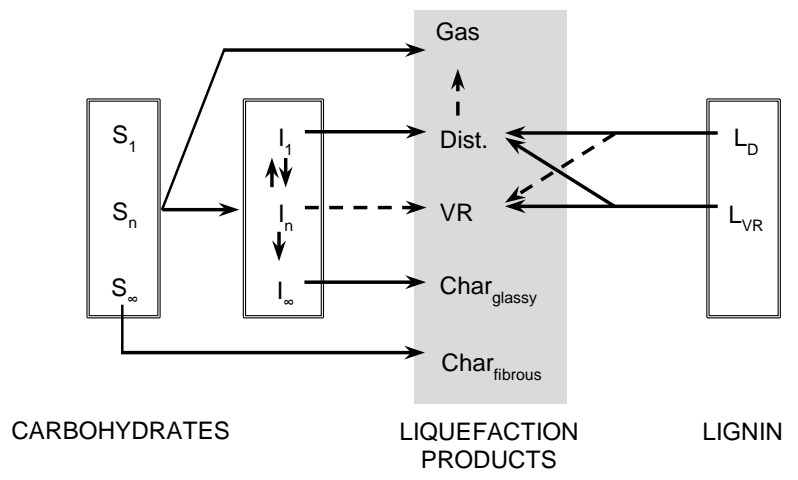

Figure 11. Reaction scheme for the liquefaction of carbohydrates and lignin. $S_{i}$ and $I_{i}$ represent sugars and intermediates with different polymerization degree. LD and LvR represent lignin with Mw in the Distillate and the VR ranges respectively. Dotted lines indicate minor reactions.

During the initial stages of the liquefaction, all carbohydrates undergo a series of polymerisation and depolymerization reactions to form a wide range of reaction intermediates. The light $\mathrm{Mw}$ intermediate would be subject to (de)polymerization equilibrium whereas the heavier components would deposit on reactor wall and stirrer and, eventually, condense and degrade into glassy char. However, the crystalline cellulose would dehydrate and condense before significant depolymerisation, leading to the formation of fibrous char. This is consistent with cellulose, amylopectin and glucose giving bio-crudes with nearly identical GPC chromatograms but different char yields and structure. 
Lignin decomposes to form practically only bio-crude. The lighter fraction of lignin (LD) will mainly form Distillate. However, some of the light lignin might also condense to form heavier species (VR). The heavier fraction of the lignin (LvR) will both undergo cracking and condensation reactions, leading to the formation of both Distillate and VR.

\section{Conclusions}

Pinewood and several model components (i.e. cellulose, amylopectin, lignin and glucose) were liquefied in 1-methylnapthalene and their products were analysed for a better understanding of the liquefaction process. The results lead to the following conclusions:

- Char has mainly a carbohydrate origin and is does not originate from the lignin nor from the lignin-like bio-crude to a significant extent.

- Cellulose, amylopectin and glucose chars have the same chemical composition but different morphology. While cellulose char presents a fibrous structure, amylopectin and glucose chars are composed of amorphous and compact particles.

- The heavy tail of the bio-crude (Vacuum Residue) originates mainly from the condensation of the lignin components via recalcitrant $\mathrm{C}-\mathrm{C}$ coupling, a reaction that proceeds in parallel to the cracking of weaker ether bridges that form smaller lignin fragments.

- Despite their structural and compositional differences, cellulose, amylopectin and lignin all yielded very similar lignin-like bio-oils that are rich in aromatic carbons ( $\sim 0 \mathrm{C} \%$ measured by $\left.{ }^{13} \mathrm{C}-\mathrm{NMR}\right)$, which covers both furanic and phenolic species (evidenced by GC-MS). All bio-oils also contain a significant fraction of paraffinic carbon $(\sim 25 \mathrm{C} \%)$ and moderate fractions of aliphatic alcohol/ether and carbonyl/carboxyl carbons ( 15 C\%). Various reaction 
sequences are proposed to explain the conversion of sugars to furanic, phenolic and paraffinic species.

- The conversion of carbohydrates proceeds with the formation of a bio-crude with a well-defined $\mathrm{Mw}$ distribution and a char yield that depends on the concentration of reaction intermediates in the liquefaction medium. Gas is mainly formed via decarboxylation/decarbonylation of the carbohydrates and the liquid products. A reaction scheme is proposed for the conversion of carbohydrates to gas, bio-crude and solid.

- The conversion of lignin proceeds via depolymerisation reactions to lighter biocrude and, to a lesser extent some parallel condensation to heavy products. The formation of gas and solid remains marginal. Lignin VR decomposes slowly and, thereby, it continues cracking to distillate when all the other reactions are finished.

- Char formation is subjected to different driving forces in liquefaction and pyrolysis, namely either the insolubility (liquefaction) or the low volatility (pyrolysis) of the heavy components under reaction condition. 


\section{References}

1. van Rossum, G., Zhao, W., Castellvi Barnes, M., Lange, J.-P., and Kersten, S.R.A., Liquefaction of Lignocellulosic Biomass: Solvent, Process Parameter, and Recycle Oil Screening. ChemSusChem, 2013. 7(1): p. $253-259$.

2. Kumar, S., Direct Liquefaction of Lignocellulose: Exploration, Design and Evaluation of Conceptual Processes, in Sustainable Process Technology. 2015, University of Twente: Netherlands. p. 166.

3. Akhtar, J. and Amin, N.A.S., A review on process conditions for optimum bio-oil yield in hydrothermal liquefaction of biomass. Renewable and Sustainable Energy Reviews, 2011. 15(3): p. 1615-1624.

4. Kumar, S., Lange, J.-P., Van Rossum, G., and Kersten, S.R.A., Liquefaction of Lignocellulose: Process Parameter Study To Minimize Heavy Ends. Industrial \& Engineering Chemistry Research, 2014. 53(29): p. 11668-11676.

5. Westerhof, R.J.M., Nygård, H.S., Van Swaaij, W.P.M., Kersten, S.R.A., and Brilman, D.W.F., Effect of particle geometry and microstructure on fast pyrolysis of beech wood. Energy and Fuels, 2012. 26(4): p. 2274-2280.

6. Piskorz, J., Radlein, D.S.A.G., Scott, D.S., and Czernik, S., Pretreatment of wood and cellulose for production of sugars by fast pyrolysis. Journal of Analytical and Applied Pyrolysis, 1989. 16(2): p. 127-142.

7. Bridgwater, A.V., Review of fast pyrolysis of biomass and product upgrading. Biomass and Bioenergy, 2012. 38: p. 68-94.

8. Mohan, D., Pittman, C.U., and Steele, P.H., Pyrolysis of Wood/Biomass for Bio-oil: A Critical Review. Energy \& Fuels, 2006. 20(3): p. 848-889.

9. Castellví Barnés, M., Lange, J.P., van Rossum, G., and Kersten, S.R.A., A neww approach for bio-oil characterization based on gel permeation chromatography preparative fractionation. Journal of Analytical and Applied Pyrolysis. 113: p. 444-453.

10. Kumar, S., Lange, J.P., Rossum, G.V., and Kersten, S.R.A., Liquefaction of Lignocellulose in Fractionated Light Bio-Oil: Proof of Concept and Techno-Economic Assessment. ACS Sustainable Chemistry and Engineering, 2015. 3(9): p. 2271-2280. 
11. Rojas, J., Lopez, A., Guisao, S., and Ortiz, C., Evaluation of several microcrystalline celluloses obtained from agricultural by-products. Journal of Advanced Pharmaceutical Technology \& Research, 2011. 2(3): p. 144-150.

12. Kersten, S. and Garcia-Perez, M., Recent developments in fast pyrolysis of ligno-cellulosic materials. Current Opinion in Biotechnology, 2013. 24(3): p. 414-420.

13. Zhou, S., Pecha, B., van Kuppevelt, M., McDonald, A.G., and Garcia-Perez, M., Slow and fast pyrolysis of Douglas-fir lignin: Importance of liquid-intermediate formation on the distribution of products. Biomass and Bioenergy, 2014. 66(0): p. 398-409.

14. Wang, Z., Pecha, B., Westerhof, R.J.M., Kersten, S.R.A., Li, C.-Z., McDonald, A.G., and Garcia-Perez, M., Effect of Cellulose Crystallinity on Solid/Liquid Phase Reactions Responsible for the Formation of Carbonaceous Residues during Pyrolysis. Industrial \& Engineering Chemistry Research, 2014. 53(8): p. 2940-2955.

15. Scott, D.S., Piskorz, J., Bergougnou, M.A., Graham, R., and Overend, R.P., The role of temperature in the fast pyrolysis of cellulose and wood. Industrial \& Engineering Chemistry Research, 1988. 27(1): p. 8-15.

16. Ben, H. and Ragauskas, A.J., Comparison for the compositions of fast and slow pyrolysis oils by NMR characterization. Bioresource Technology, 2013. 147(0): p. 577-584.

17. Oudenhoven, S.R.G., Westerhof, R.J.M., Aldenkamp, N., Brilman, D.W.F., and Kersten, S.R.A., Demineralization of wood using wood-derived acid: Towards a selective pyrolysis process for fuel and chemicals production. Journal of Analytical and Applied Pyrolysis, 2013. 103(0): p. $112-118$.

18. Mettler, M.S., Mushrif, S.H., Paulsen, A.D., Javadekar, A.D., Vlachos, D.G., and Dauenhauer, P.J., Revealing pyrolysis chemistry for biofuels production: Conversion of cellulose to furans and small oxygenates. Energy \& Environmental Science, 2012. 5(1): p. 5414.

19. Antal Jr, M.J., Mok, W.S.L., and Richards, G.N., Mechanism of formation of 5(hydroxymethyl)-2-furaldehyde from d-fructose and sucrose. Carbohydrate Research, 1990. 199(1): p. 91-109.

20. Antal Jr, M.J., Leesomboon, T., Mok, W.S., and Richards, G.N., Mechanism of formation of 2-furaldehyde from d-xylose. Carbohydrate Research, 1991. 217(0): p. 71-85.

21. Scheirs, J., Camino, G., and Tumiatti, W., Overview of water evolution during the thermal degradation of cellulose. European Polymer Journal, 2001. 37(5): p. 933-942. 
22. Luijkx, G.C.A., van Rantwijk, F., and van Bekkum, H., Hydrothermal formation of 1,2,4benzenetriol from 5-hydroxymethyl-2-furaldehyde and d-fructose. Carbohydrate Research, 1993. 242(0): p. 131-139.

23. Pastorova, I., Botto, R.E., Arisz, P.W., and Boon, J.J., Cellulose char structure: a combined analytical Py-GC-MS, FTIR, and NMR study. Carbohydrate Research, 1994. 262(1): p. 2747.

24. Catallo, W.J., Shupe, T.F., Comeaux, J.L., and Junk, T., Transformation of glucose to volatile and semi-volatile products in hydrothermal (HT) systems. Biomass and Bioenergy, 2010. 34(1): p. 1-13.

25. Cheng, Y.-T. and Huber, G.W., Production of targeted aromatics by using Diels-Alder classes of reactions with furans and olefins over ZSM-5. Green Chemistry, 2012. 14(11): p. 3114-3125.

26. Vaitheeswaran, S., Green, S.K., Dauenhauer, P., and Auerbach, S.M., On the Way to Biofuels from Furan: Discriminating Diels-Alder and Ring-Opening Mechanisms. ACS Catalysis, 2013. 3(9): p. 2012-2019.

27. Antal Jr, M.J., Mok, W.S.L., and Richards, G.N., Four-carbon model compounds for the reactions of sugars in water at high temperature. Carbohydrate Research, 1990. 199(1): p. 111115.

28. Girisuta, B., Janssen, L.P.B.M., and Heeres, H.J., Green Chemicals: A Kinetic Study on the Conversion of Glucose to Levulinic Acid. Chemical Engineering Research and Design, 2006. 84(5): p. 339-349.

29. Wang, Z., Pecha, B., Westerhof, R.J.M., Kersten, S.R.A., Li, C.Z., McDonald, A.G., and Garcia-Perez, M., Effect of cellulose crystallinity on solid/liquid phase reactions responsible for the formation of carbonaceous residues during pyrolysis. Industrial and Engineering Chemistry Research, 2014. 53(8): p. 2940-2955.

30. Patil, S.K.R. and Lund, C.R.F., Formation and Growth of Humins via Aldol Addition and Condensation during Acid-Catalyzed Conversion of 5-Hydroxymethylfurfural. Energy \& Fuels, 2011. 25(10): p. 4745-4755.

31. Patil, S.K.R., Heltzel, J., and Lund, C.R.F., Comparison of Structural Features of Humins Formed Catalytically from Glucose, Fructose, and 5-Hydroxymethylfurfuraldehyde. Energy \& Fuels, 2012. 26(8): p. 5281-5293. 
32. van Zandvoort, I., Wang, Y., Rasrendra, C.B., van Eck, E.R.H., Bruijnincx, P.C.A., Heeres, H.J., and Weckhuysen, B.M., Formation, Molecular Structure, and Morphology of Humins in Biomass Conversion: Influence of Feedstock and Processing Conditions. ChemSusChem, 2013. 6(9): p. 1745-1758.

33. Hoang, T.M.C., van Eck, E.R.H., Bula, W.P., Gardeniers, J.G.E., Lefferts, L., and Seshan, K., Humin based by-products from biomass processing as a potential carbonaceous source for synthesis gas production. Green Chemistry, 2015. 17(2): p. 959-972.

34. Sevilla, M. and Fuertes, A.B., The production of carbon materials by hydrothermal carbonization of cellulose. Carbon, 2009. 47(9): p. 2281-2289.

35. Sevilla, M. and Fuertes, A.B., Chemical and structural properties of carbonaceous products obtained by hydrothermal carbonization of saccharides. Chemistry - A European Journal, 2009. 15(16): p. 4195-4203.

36. Barbier, J., Charon, N., Dupassieux, N., Loppinet-Serani, A., Mahé, L., Ponthus, J., Courtiade, M., Ducrozet, A., Quoineaud, A.-A., and Cansell, F., Hydrothermal conversion of lignin compounds. A detailed study of fragmentation and condensation reaction pathways. Biomass and Bioenergy, 2012. 46(0): p. 479-491.

37. Ben, H. and Ragauskas, A.J., NMR Characterization of Pyrolysis Oils from Kraft Lignin. Energy \& Fuels, 2011. 25(5): p. 2322-2332.

38. Britt, P.F., Buchanan, A.C., Cooney, M.J., and Martineau, D.R., Flash Vacuum Pyrolysis of Methoxy-Substituted Lignin Model Compounds. The Journal of Organic Chemistry, 2000. 65(5): p. 1376-1389. 


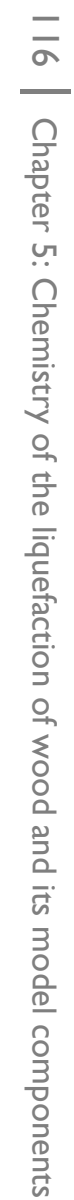




\section{Chapter 6}

Effect of the reaction medium during

liquefaction of lignocellulose 
The role of the liquefaction medium was studied by processing pinewood in various organic solvents and refinery streams at $310^{\circ} \mathrm{C}$. The resulting bio-crude was analysed with GPC and GC-MS, the solid residue with FTIR and HR-SEM, and the gas with GC. It was observed that all solvents dissolve the bio-crude and that char is not formed via bio-crude condensation. Results indicate that both char and biocrude formation occur in parallel at the biomass-solvent interphase, and that char yield is decreased by increasing the interaction between solvent and biomass. For instance, char yield is minimised for Hildebrand values of $25-40 \mathrm{MPa}^{1 / 2}$ and small Hansen distances (Ra) to cellulose and lignin. A decrease in the molecular weight of the liquefaction medium also leads to a (slight) decrease in char yield. The presence of alcohol function in the liquefaction medium resulted in lower wood conversion rates, besides the low char yield that results from its beneficial contribution to the Hildebrand or Hansen distance of the medium. 


\section{Introduction}

In chapter 3 and 4 we observed that different product distributions and bio-crude compositions are obtained when different liquefaction media are used. Liquefaction research has been performed in a wide range of solvents (see table A1 in Supporting information) [1-3]. However, most studies are performed with a very limited selection of solvents and do not focus on the effect of the liquefaction medium on the product distribution or the bio-crude quality. Few publications tried to relate the solvent properties with the product distribution and the liquefaction kinetics. Stevens et al. [4] for instance, reported that solvents with Hansen distance (Ra) to coniferyl alcohol smaller than $15 \mathrm{MPa}^{1 / 2}$ led to low solid yields $(<10 \%)$ as they allow a good dissolution of the bio-crude and the early biomass liquefaction products. Heitz et al. [5] studied lignocellulose liquefaction at various temperatures (reaction severity) in different types of solvent. They observed a significant effect of the solvent on the extent of the liquefaction of wood, and proposed that alcohols have a protective effect on the wood matrix, decreasing the wood liquefaction rate.

In the current chapter, we investigated the effect of the liquefaction medium on the liquefaction of wood. A wide range of solvents and solvent mixtures, with different chemical functionalities and molecular weights were tested as liquefaction media. Afterwards, the bio-crude, the solid and the gas products were quantified and characterised by various techniques (GPC, GC, GC-MS, elemental analysis (EA), FTIR and HR-SEM). The aim of the study was not only to find a good liquefaction solvent, but also to understand which solvent properties guarantee a good biomass decomposition into bio-crude and prevent char formation. 


\section{Results}

\section{I. Liquefaction in single solvents}

Initial experiments were performed for $30 \mathrm{~min}$ at $310^{\circ} \mathrm{C}$ with $10 \mathrm{wt} \%$ of dry wood and $90 \mathrm{wt} \%$ of solvent intake. The various liquefaction media yielded a wide range of product yields, with char yields varying between 0 and $57 \mathrm{C} \%$, the gas yields between 3 and $31 \mathrm{C} \%$, and the bio-crude yields between 40 and $94 \mathrm{C} \%$ (Figure 2).

Among the hydrocarbons, aromatic solvents (e.g. methylnaphthalene) led to higher bio-crude yields (i.e. VR + Distillate) than paraffinic solvents (e.g. decalin or nundecane). However, the presence of oxygenated groups in the solvent (e.g. phenol or anisol) seemed to further increase the bio-crude yield. This agrees with the results reported in chapter 3 [6], which showed that oxygenated and polar solvents give higher bio-crude yields that non-oxygenated solvents. The impact of the liquefaction medium properties on the bio-crude and solid yields will be discussed in detail later (section 3.1).

Pyrogallol showed remarkably high solid and gas yields. This is due to its reactivity under the reaction conditions, for a blank experiment (100 $\mathrm{wt} \%$ of pyrogallol) yielded $4 \mathrm{C} \%$ of char and $1 \mathrm{C} \%$ of gas (Table H3 and Figure H1a, Supporting information). Therefore, pyrogallol was not considered for further studies.

The liquefaction reaction appeared to result in one of two types of solid residue, based on FTIR analysis [7] (chapter 5). Solvents with hydroxyl groups resulted in a solid residue that resembled unconverted wood whereas the other solvents resulted in an aromatic char-like solid. In addition, SEM images showed that both unconverted wood and char solids retained the fibrous structure of the initial biomass. This suggests that the char is formed via direct dehydration of the wood fibres rather than by condensation of bio-crude products into insoluble polymer. Further details on the solid characterization can be found in the Supporting information (section G). 
The presence of alcohol groups in the liquefaction medium had a remarkable effect on the wood conversion rate. Liquefaction times of 20-30 minutes were sufficient to achieve complete conversion in the absence of alcohol groups. However, in the presence of an alcohol, the conversion rate decreased and led to the presence of unconverted wood after 30 minutes of liquefaction (see Figure 1 and Figure 2). Figure 1 shows that phenol liquefaction delivered cellulose-like solid after 60 minutes of liquefaction, and guaiacol liquefaction was not complete even after 120 minutes of reaction.

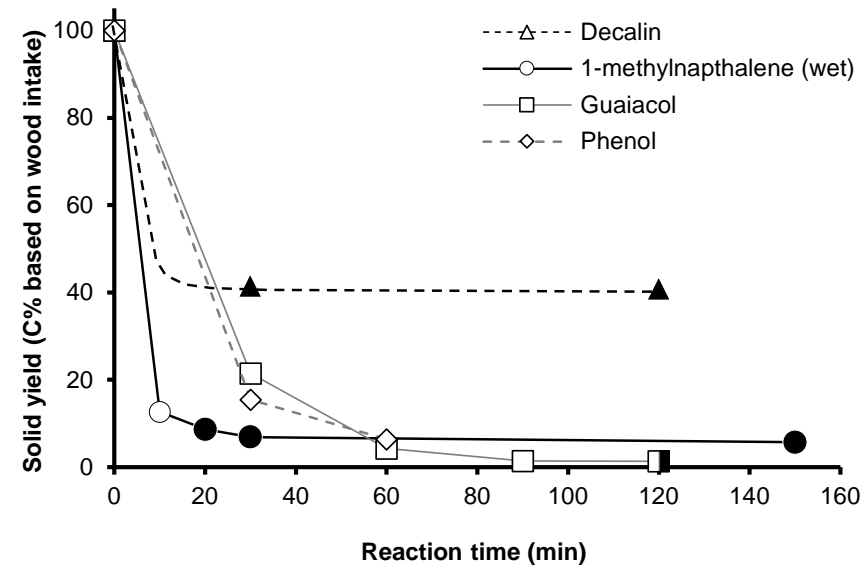

Figure 1. Effect of reaction time on solid yield, with residual solid being mainly unconverted wood (open symbols) or char (closed symbols). Guaiacol [6], decalin and phenol experiments performed with $90 \mathrm{wt} \%$ of solvent and $10 \mathrm{wt} \%$ of wood at $310^{\circ} \mathrm{C}$. 1methylnaphthalene experiments performed with $85 \mathrm{wt} \%$ of 1methylnaphthalene, $5 \mathrm{wt} \%$ of water and $10 \mathrm{wt} \%$ of wood at $310^{\circ} \mathrm{C}$.

It should be stressed that the yields reported with char-type residue represent ultimate yields at full conversion. In contrast, the yields observed in runs that deliver cellulose-like residue represent yields at partial wood conversion. Indeed, earlier studies carried out at various reaction times in various solvents (guaiacol or 1methylnaphthalene (chapter 5) [8]) showed a steady increase of gas and bio-crude 
yield and decreasing solid yield with time as long as the cellulosic solid features in FTIR. Once these features disappeared, the gas, bio-crude and char yields remained generally constant even at extended reaction times. This behaviour was confirmed here for a number of solvents, namely guaiacol, decalin, 1-methylnapthalene and various refinery streams (Figure 1, and Figure H2 in Supporting information).

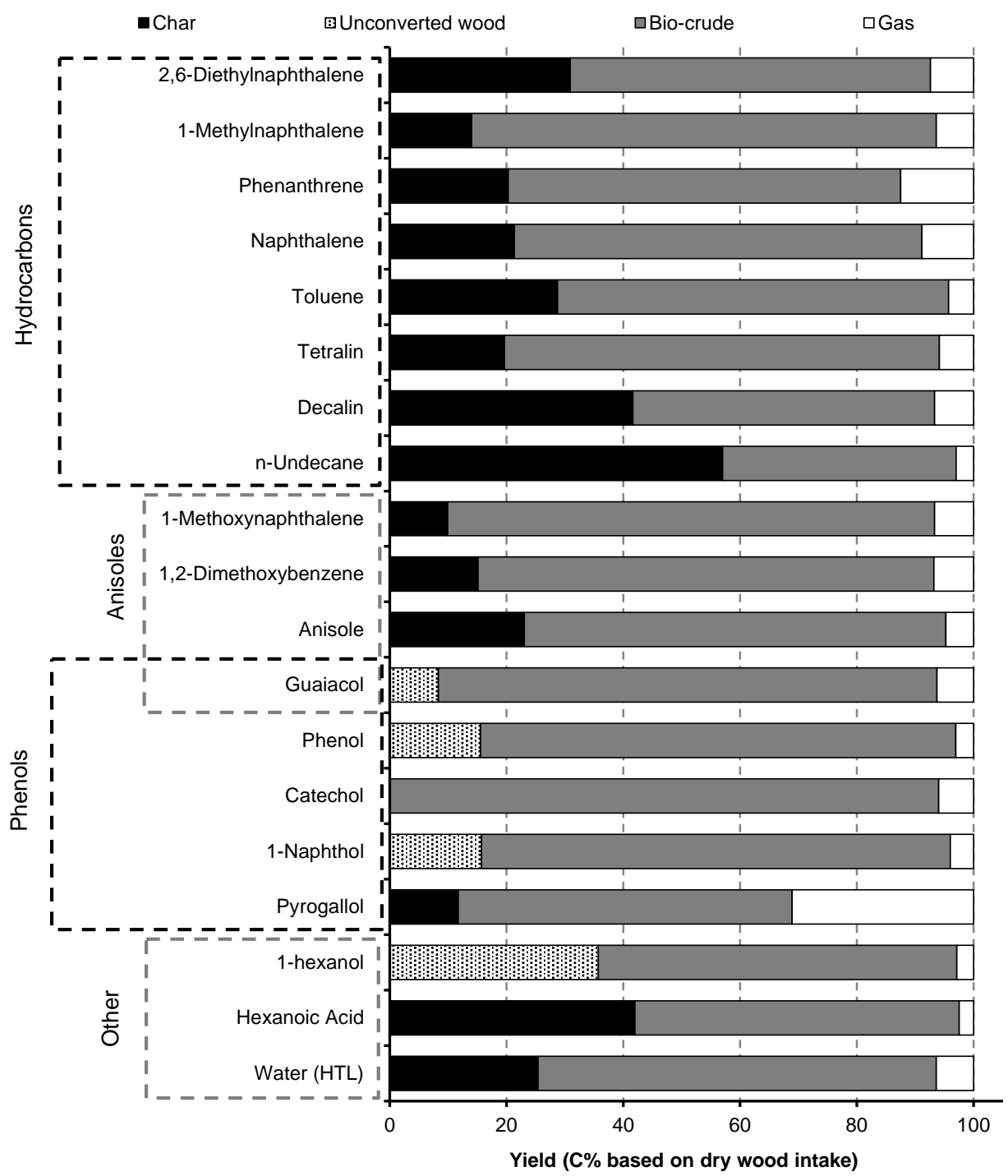

Figure 2. Product yields obtained in various liquefaction media. Experiments performed at $310^{\circ} \mathrm{C}$ for $30 \mathrm{~min}$ and with $10 \mathrm{wt} \%$ of wood and $90 \mathrm{wt} \%$ of solvent intake. 


\subsection{Liquefaction in refinery streams}

Liquefaction was also performed using four refinery streams with different $\mathrm{Mw}$ and degree of aromaticity, namely cracked gasoil (CGO), light cycle oil (LCO), vacuum gasoil (VGO) and Hydrowax. A wide range of char yields was obtained (Figure 3), with CGO giving the lowest char yield (2 C\%) and Hydrowax the highest $(60 \mathrm{C} \%)$. Consistently with the observation made above, a higher degree of aromaticity (percentage of $\mathrm{sp}_{2}$ carbon) of the liquefaction solvent seemed to result in lower char yields (Figure 3). It is worth mentioning though, that the blank experiment performed with CGO revealed the formation of heavy liquid species (Figure H1b, Supporting information), indicating that CGO is not stable under liquefaction conditions. Thus, CGO was not considered for further studies.

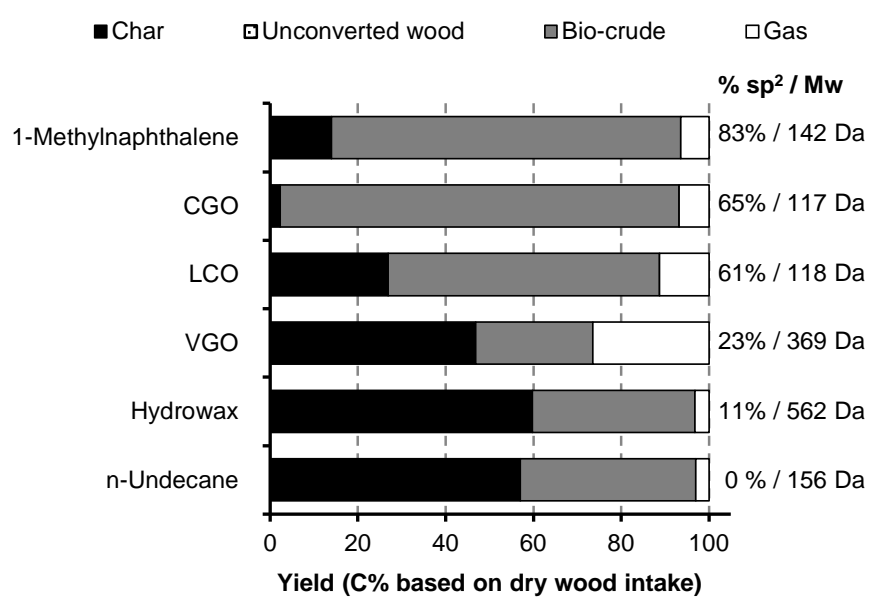

Figure 3. Product yields obtained in refinery streams. Experiments performed at $310^{\circ} \mathrm{C}$ for $30 \mathrm{~min}$ and with $10 \mathrm{wt} \%$ of wood and $90 \mathrm{wt} \%$ of solvent intake (key characteristics of the solvents are reported beside the table, namely fraction of $\mathrm{sp}_{2}$ carbon and $\mathrm{Mw}$ ). 


\subsection{Liquefaction in solvent mixtures}

Additional experiments were also performed using solvent mixtures. Figure 4 shows that the liquefaction in solvent mixtures generally leads to lower solid yields than the liquefaction in single solvents. According to Figure 4, the addition of water improves the liquefaction in all cases, i.e. when the solid residue is either unconverted wood (e.g. guaiacol) or char (e.g. 1-methylnaphthalene). The former case indicates an acceleration of the liquefaction reaction while the latter suggests in deeper ultimate liquefaction. These results are discussed in more detail in the discussion (section 3.2).

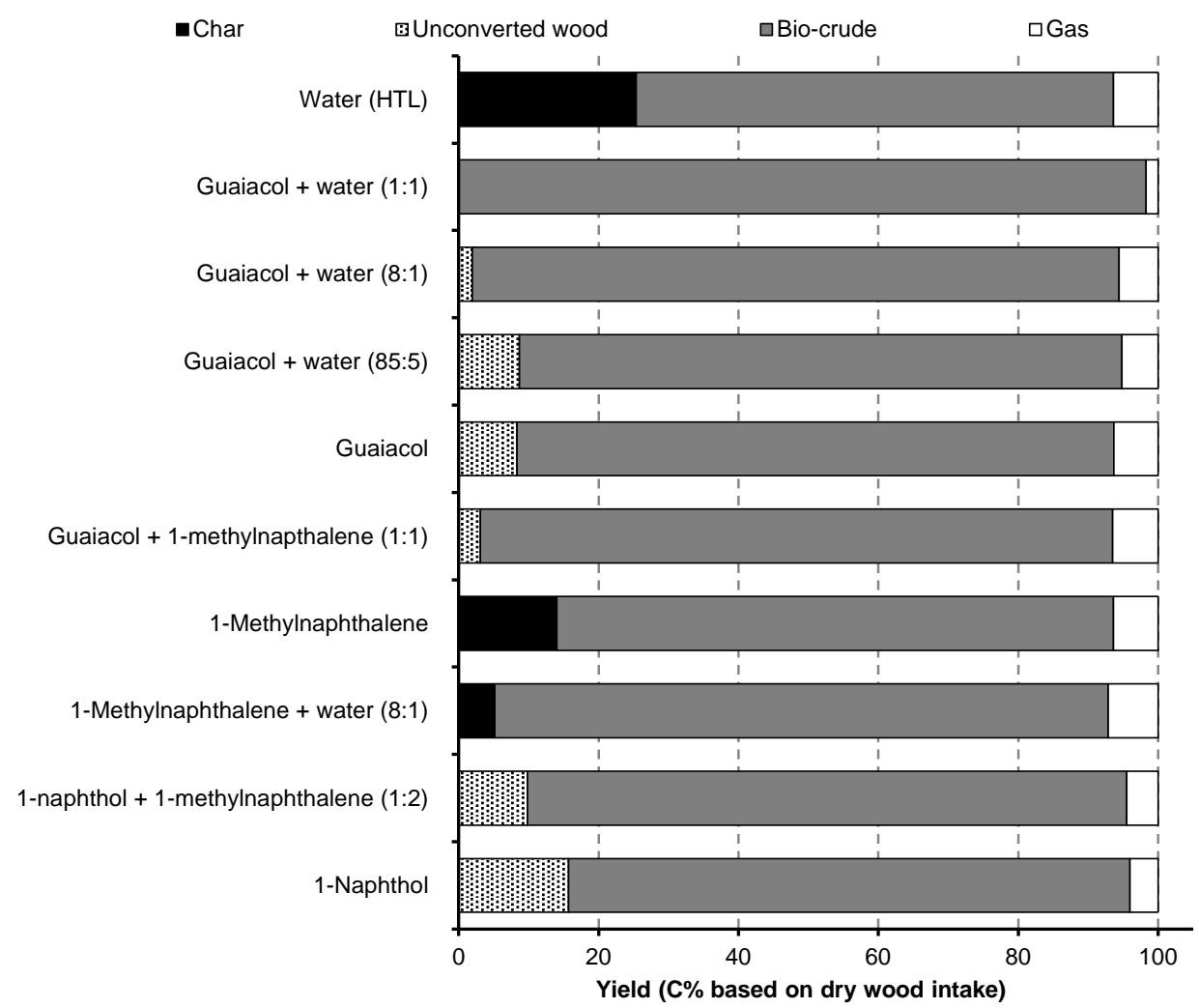

Figure 4. Product yields obtained in solvent mixtures (single solvents showed as reference). Experiments performed at $310^{\circ} \mathrm{C}$ for $30 \mathrm{~min}$ and with $10 \mathrm{wt} \%$ of wood and $90 \mathrm{wt} \%$ of solvent intake (the solvent weight ratio is reported between brackets). 
Results shown in Figure 4 confirm that the presence of alcohols in the liquefaction medium results in lower conversion rates, as solvents that tend to give char after 30 minutes of liquefaction, provide unconverted wood after the addition of a phenol (water or methylnaphthalene after the addition of guaiacol or Naphthol).

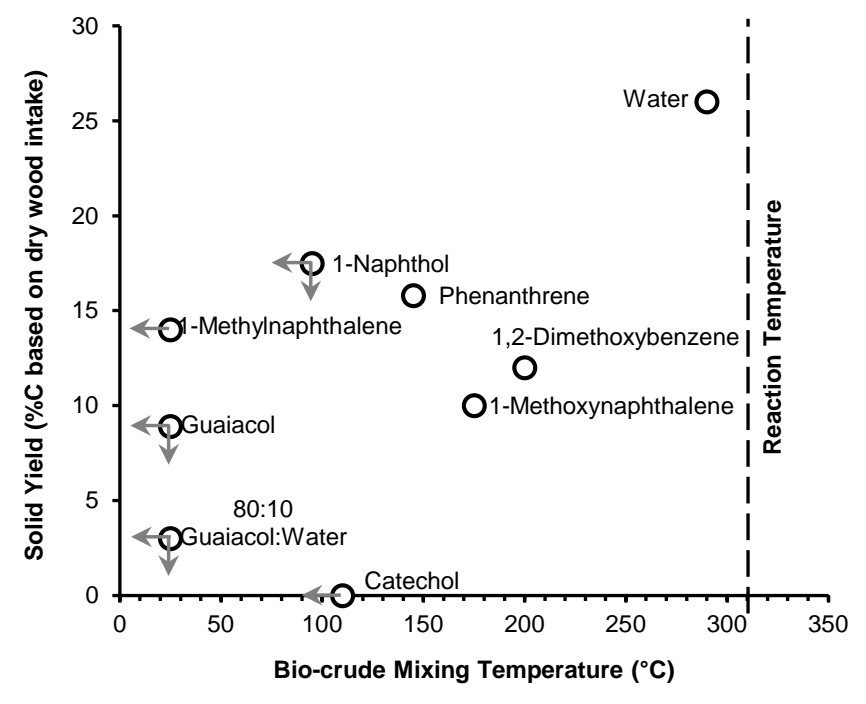

Figure 5. Results of the solubility tests in glass capillaries. The arrows pointing down indicate that the solid yield may vary if complete wood conversion is achieved. The arrows pointing to the left indicate that the bio-crude is likely soluble at lower temperature than measured, as the measured temperature is either room temperature or the melting temperature of the solvent.

\subsection{Solubility experiments in capillaries}

Solubility experiments were performed to determine whether the formation of char was caused by a poor solubility of the bio-crude in the liquefaction medium. For these experiments, bio-crude and a solvent were introduced in a glass capillary, which was then sealed and heated at the liquefaction temperature $\left(\geq 300^{\circ} \mathrm{C}\right)$. Under way, attention was paid at the mixing behaviour in attempt to determine the 
temperature at which the bio-crude dissolved in the solvent. Results showed that at reaction conditions bio-crude was soluble in all the solvents. Furthermore, no correlation was found between the minimum mixing temperature and the product yields (Figure 5).

\section{Discussion}

\section{I. Effect of solvent parameters on product distribution}

The results presented in Figure 2, Figure 3 and Figure 4 clearly show that the liquefaction medium has an important effect on the product distribution.

It is frequently reported that, in liquefaction, the main role of the solvent is to dissolve and disperse the bio-crude produced to prevent char formation $[9,10]$. If the solvent does not dissolve the bio-crude properly, the insoluble molecules will aggregate and, eventually, repolymerise to char. This effect was not observed for the solvents and experimental conditions presented in this work. The solubility experiments presented in section 2.4 showed that, under liquefaction conditions (temperature $\geq 300^{\circ} \mathrm{C}$ ) all solvents dissolve the bio-crude. In addition, the char-like solid residue obtained in the experiments presented a fibrous structure, indicating that char is formed via direct dehydration of the initial fibrous structure rather than from the condensation of bio-crude products.

Thus, the effect of the solvent must be associated with the initial decomposition of the biomass building blocks. Two possible hypotheses are considered, based on the product distributions obtained for various solvents, and the effect of the alcohol groups on the type of solid residue and wood conversion rate.

In the first hypothesis, the wood (the cell wall) maintains its rigid structure during liquefaction. As a consequence, the molecular segments of the cellulose are tight up by the crystal structure of the cellulose and, thereby, cannot freely rearrange and degrade into char. Thus, both biomass decomposition and char formation can only 
occur on the surface of the cell wall. During liquefaction, the dehydration and charring of the biomass compete with the cleavage and dissolution of the initial lignocellulose decay products. Accordingly, a good affinity of the liquefaction medium with the biomass polymers should lead to high bio-crude and low char yields.

In the second hypothesis, wood decomposition and char formation occur throughout the cell wall, but are still slowed down by the presence of solvents with alcohol groups. Accordingly, the solvent is penetrating deep inside the cell wall, e.g. through swelling, preventing char formation both at the surface, and inside the cell wall. As proposed by Heitz et al. [5], this would be possible if the liquefaction temperature was higher than the glass transition points of the wood polymers, making wood less rigid and more accessible to solvent wetting.

Based on the obtained results and the considered hypotheses, we propose the reaction scheme depicted in Figure 6 to describe wood liquefaction. Under the studied conditions, char is not formed from the condensation of bio-crude, as indicated by its fibrous structure (section $\mathrm{G}$ in Supporting information) and the biocrude stability at extended liquefaction times (Figure H2, Supporting information). Besides, char is probably not formed directly from the biomass, but from the initial biomass decay products (referred to as Intermediate in Figure 6) formed at the biomass-solvent interphase. In both hypotheses, char formation occurs at the vicinity of the liquefaction solvent when the solvent is not sufficiently efficient in dissolving the initial biomass decomposition products and removing them from the remaining wood fibres. Therefore, char yields are expected to be related to the solubility properties of the liquefaction medium. 


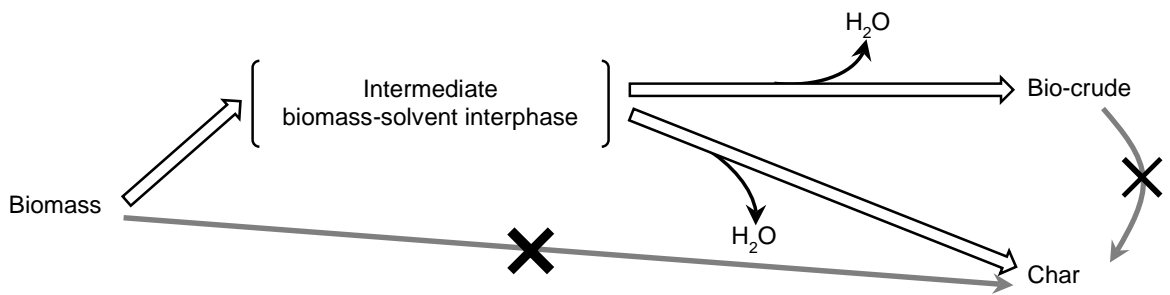

Figure 6. Reaction scheme for the liquefaction of wood.

Figure 7 and Figure 8 show how the solubility parameters Hildebrand and Hansen distance ( $\mathrm{Ra}$ ) are indeed related to the solid yield (obtained for liquefaction times of 30 minutes). Figure 7 shows that the solid yield reaches a minimum at intermediate Hildebrand values of 25-40 $\mathrm{MPa}^{1 / 2}$ (Figure 7). As reported by Hildebrand et al. [11], components with similar Hildebrand parameter should have a good interaction. Hence, it is reasonable to expect that the solvents with Hildebrand closer to cellulose and lignin lead to lower solid yields.
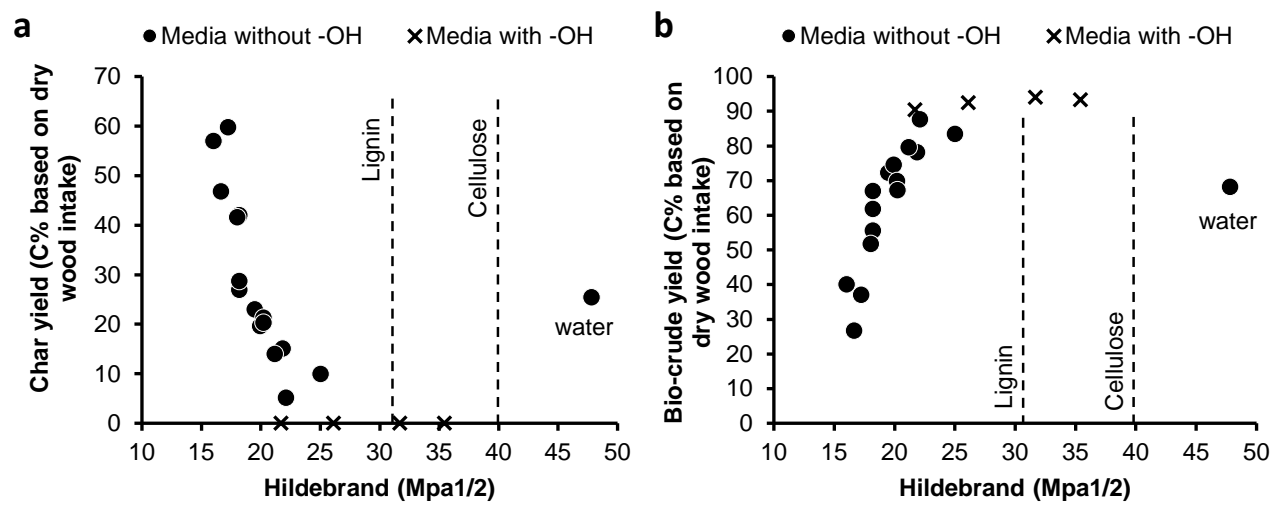

Figure 7. Effect of the Hildebrand parameter of the liquefaction media on the (a) char and (b) bio-crude yields. Only experiments with wood conversion $>95 \mathrm{C} \%$ are represented. Char yield of media with $-\mathrm{OH}$ is set to zero because the solid residue consists mainly of unconverted wood and is already $<5 \mathrm{C} \%$.

It is important to stress that the graphs presented in Figure 7 and Figure 8 show solid yields obtained at complete biomass conversion (conversion above $95 \mathrm{C} \%$ ). As 
showed in the results and discussed in the following section (3.2), alcohol groups in the liquefaction media slow down wood conversion. However, product distribution at full conversion was not known for all those solvents and, therefore, it cannot be concluded whether the formation of char is completely prevented, leading to $0 \mathrm{C} \%$ char yield at full conversion; or only slowed down, leading to char yields determined by the solubility parameters of the liquefaction media.
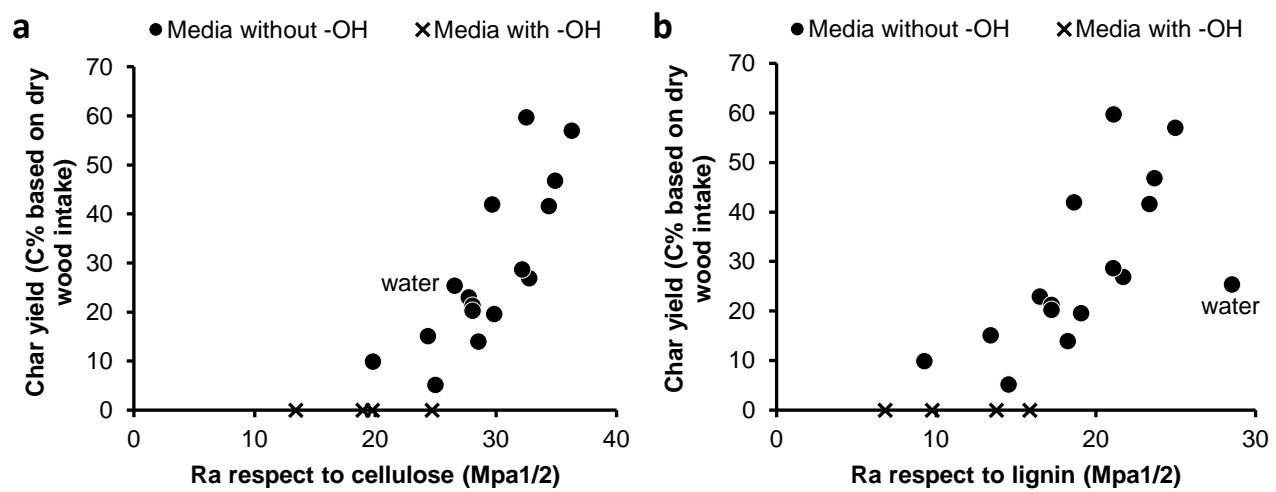

Figure 8. Correlations between solid yield and Ra of the liquefaction media respect to (a) cellulose and (b) lignin. Only experiments with wood conversion $>95 \mathrm{C} \%$ are represented. Char yield of media with $-\mathrm{OH}$ is set to zero because the solid residue consists mainly of unconverted wood and is already $<5 \mathrm{C} \%$.

However, one should bear in mind that the Hildebrand parameter does not differentiate between the various types of interaction. This shortcoming can be alleviated by using the Hansen solubility parameters, which attempt to decompose the Hildebrand parameter in three main constituting interactions, namely dispersion, dipolar and hydrogen bonding interactions (see section 6.4 in chapter 2). Thus, we calculated the Hansen distances ( $\mathrm{Ra}$ ) between cellulose and all the liquefaction media, and correlated them with the solid yields. A trend was observed, where the solid yield appeared to increase with the Hansen distance Ra (Figure 8). A similar trend was obtained with the Hansen distance Ra to lignin, although water 
behaved as an outlier in that case, as it gave less solid residue than expected from the Ra distance (Figure 8).

The results reported in chapter 5 revealed that, in liquefaction, carbohydrates are the main source for char formation, while the liquefaction of lignin leads to very low char yields. This suggests that char formation is mainly determined by the interaction between the liquefaction medium and the carbohydrates of the wood.

Interestingly, the solubility parameters appeared also to rationalise the beneficial effect of adding water to the medium or adding phenolic solvents to hydrocarbon solvents. The addition of a more polar solvent to a liquefaction medium resulted in a medium with an increased Hildebrand parameter, or a decreased Hansen distance, thus, with a better interaction with the wood components. This effect can be observed with the mixtures of 1-methylnaphthalene with guaiacol or 1-naphthol (see Figure 4). The addition of water on the liquefaction medium also resulted in an increase in biomass conversion [7] (see guaiacol liquefaction with and without water, Figure 4), suggesting that water acts as a catalyst and increases the conversion rate. This was already reported by Kumar et al. [8], who observed that, for liquefaction in guaiacol, higher water concentrations increased wood conversion without altering the product selectivity. Similar results have been reported in literature $[4,6,8]$.

It is worth mentioning that a relation between the solid yields and the dielectric constant of the liquefaction media was observed (Figure H5, Supporting information). These results were not surprising, as Darby et al. reported that Hildebrand $(\delta)$ and dielectric constant $(\varepsilon)$ are related through the equation: $\delta=7^{*} \varepsilon$ [12].

It is reported in literature that the molecular size of a solvent has an important effect on its solvation capacity [13]. Smaller species are generally better solvents and can diffuse more easily through polymeric structures. Therefore, we studied the effect of solvent $\mathrm{Mw}$ on the solid yield by comparing the results obtained with various 
hydrocarbons and refinery streams, which offer a significant variety of Mw. A linear regression analysis of the data showed that, besides Hildebrand or Ra, also Mw has a small but significant effect on the solid yield (p-value of 0.006 for $\mathrm{Mw}$ and 0.0008 for Ra; Table H4, Supporting information). Figure 9 shows the correlation between the measured solid yields and the solid yields predicted by the linear correlation.

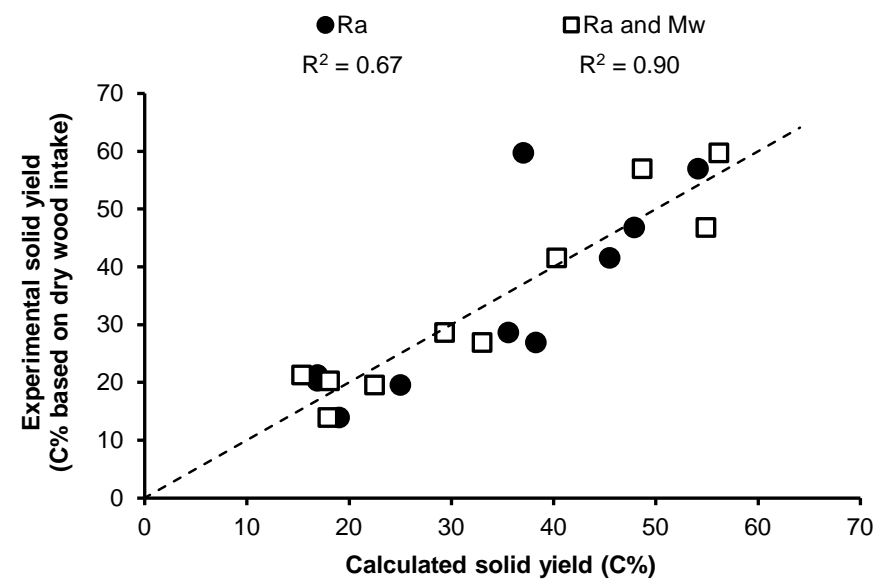

Figure 9. Effect of Ra distance to cellulose and Mw on the solid residue yield. Only refinery streams and hydrocarbons are represented.

The results presented in the current section have been compared with the work reported by Stevens et al. [4] and Heitz et al. [5]. Stevens et al. [4] reported that liquefaction media with Ra distances to coniferyl alcohol smaller than $15 \mathrm{MPa}^{1 / 2}$ led to solid yields below $10 \mathrm{wt} \%$. Although our results agree that lower solid yields are generally obtained for lower Raconiferyl alcohol (Figure H4, Supporting information), the observed trend showed considerable scattering and had several outliers (solid yield $>10 \mathrm{wt} \%$ for Raconiferyl alcohol $<15 \mathrm{MPa}^{1 / 2}$ ). We consider more appropriate the use of the Ra distance to cellulose or lignin to evaluate whether a liquefaction medium will lead to high or low solid yields, as their interaction (or the interaction of their immediate decay products) with the liquefaction media is what determines the product distribution. Heitz et al. [5] also looked at the role of solubility parameter in 
liquefaction. However, they were operating at partial conversion and, thereby, unravelled kinetic effects rather than ultimate yields at full conversion.

\subsection{Kinetics of liquefaction}

Earlier studies (chapter 5) showed that conversion of wood proceeds via monotonous decrease of solid residue as long as it shows a cellulose-like structure in FTIR. Once the remaining solid shows mainly char like structure, its residual yield remains constant even after extended reaction time. This trend was confirmed here for a number of solvents, namely decalin, 1-methylnaphthalene, guaiacol, CGO, LGO and hydrowax (Figure 1, and Figure H2 in Supporting information).

We showed that all hydrocarbon solvents as well as methoxy-benzene solvents reached the stage of char solid within $30 \mathrm{~min}$ at $310^{\circ} \mathrm{C}$. In the case of methylnaphthalene, reaction times lower than $10 \mathrm{~min}$ were needed to still detect unconverted wood in the solid (Figure 1). In contrast, phenolic solvents as well as 1hexanol needed more than $30 \mathrm{~min}$ to reach the stage of char residue. For instance, guaiacol, needed $120 \mathrm{~min}$, and phenol more than $60 \mathrm{~min}$ (Figure 1). The addition of guaiacol or naphthol to methylnaphthalene also left unconverted cellulose after 30 min. Hence, we can conclude that alcohol groups slow down the conversion of the wood [5], arguably by strongly interacting (and possibly reacting) with the cellulose and protecting it against liquefaction and charring.

This effect of hydroxyls contrasts with the effect of water, which can be seen as fast solvent [5]. Indeed, water led to char residue within $30 \mathrm{~min}$. The addition of water to a 'slow' solvent such as guaiacol led to lower residual unconverted wood, which suggests a rate enhancement. Interestingly, the addition of water to a 'fast' solvent such as methylnaphthalene resulted in lower char yield. This can't be rationalized in terms of faster kinetics but should be attributed to improved solubilisation of reaction intermediates, e.g. through reduced overall Ra distance, as discussed earlier. 
Reaction rates in the various liquefaction solvents was not determined as this chapter does not focus on the kinetics of the liquefaction process. Furthermore, the full conversion obtained for most of the solvents prevented the proper determination of the reaction rate.

Table 1. Percentage of solvent recovered after liquefaction in various media.

\begin{tabular}{ll} 
Solvent & $\%$ Solvent recovered \\
1-Naphthol & 69,9 \\
\hline Catechol & 76,9 \\
\hline Phenol & 86,3 \\
\hline Guaiacol & 89,3 \\
\hline 1,2-Dimethoxybenzene & 89,4 \\
\hline Toluene & 90,2 \\
\hline Anisole & 91,2 \\
\hline Tetralin & 93,1 \\
\hline 1-Methoxynaphthalene & 94,8 \\
\hline 1-Methylnaphthalene & 94,9 \\
\hline Naphthalene & 95,8 \\
\hline Decalin & 97,4 \\
\hline Phenanthrene & 98,9 \\
\hline 2,6-Diethylnaphthalene & 101,1 \\
\hline
\end{tabular}

\subsection{Solvent Reactivity}

We observed that some of the liquefaction experiments showed moderate to poor solvent recovery (Table 1), as indicated by a significant drop of the 'solvent' peak in the GPC trace of the liquefaction product. These results indicate that some liquefaction solvents reacted and were incorporated in to the bio-crude, even though they were found stable in a blank run under liquefaction conditions. Phenolic solvents seemed to exhibit the highest reactivity, with naphthol and catechol giving the lowest solvent recovery at $70 \%$ and $77 \%$, respectively.

It is worth stressing that the eventual conversion of solvent into heavier products does not change the discussion made so far. Indeed, the bio-crude yield was 
calculated as being the wood intake minus char and gas and not by using the area of bio-crude-range product seen by GPC. However, the reactivity of the solvent is severely hindering the quantification of heavy species using GPC analysis. Hence, we could not identify reliable and conclusive effect of solvent on the formation of heavy bio-crude.

\section{Conclusions}

The role of the reaction medium during liquefaction was studied by treating pine wood in several solvents. The results led to the following conclusions:

- $\quad$ Solid yields, ranging from 0 to $57 \mathrm{C} \%$, are determined by the biomass-medium interaction (in terms of Hildebrand parameter or Hansen distance Ra) and, to a lesser extent, by the $\mathrm{Mw}$ of the liquefaction medium. A Hildebrand value of 25$40 \mathrm{MPa}^{1 / 2}$ or a small Hansen distance between the liquefaction medium and the sugars or lignin in the biomass enhance the dissolution of the initial decay products and prevent their recombination with the biomass to form char. The addition of solvents with high Hildebrand to solvents with low Hildebrand enhances this interaction. Thus, addition of water or phenols to aromatic media generally decreases char yield.

- All solvents are able to dissolve the liquefaction products under reaction conditions.

- Water enhances the wood conversion rate, but phenols and other alcohols are rate depressing. Thus, liquefaction media containing alcohols might lead to incomplete biomass conversion and, therefore, require longer reaction times to achieve complete decomposition. This effect is not yet fully understood and warrant further studies.

- Wood liquefaction and charring occur at the biomass-solvent interphase, as competing parallel reactions. However, it is not known yet if conversion occurs 
only and the surface of the cell wall or if solvent swelling allows wood conversion deep inside the cell wall.

- Phenolic solvents tend to degrade and incorporate into the bio-crude during liquefaction.

- Based on these conclusions, the perfect liquefaction medium should have a good interaction with the biomass and/or its initial decay products, and a relatively low $\mathrm{Mw}$ to minimise char formation. Typical solvents that give low char yields are aromatics containing oxygenated substituents (phenols or anisoles). However, one must keep in mind that a phenolic medium slows down wood decomposition and, therefore, requires longer reaction times. The liquefaction medium could be composed of a mixture of solvents and should contain low concentrations of water (high concentrations would lead to high pressures) to increase the wood conversion rate. This water could be already contained in the (wet) biomass, or added as a solvent. 


\section{References}

1. Bouvier, J.M., Gelus, M., and Maugendre, S., Wood liquefaction-An overview. Applied Energy, 1988. 30(2): p. 85-98.

2. Kumar, S., Direct Liquefaction of Lignocellulose: Exploration, Design and Evaluation of Conceptual Processes, in Sustainable Process Technology. 2015, University of Twente: Netherlands. p. 166.

3. Huang, H.-j. and Yuan, X.-Z., Recent progress in the direct liquefaction of typical biomass. Progress in Energy and Combustion Science, 2015. 49: p. 59-80.

4. Stevens, J., Young, M., EUHUS, D., COULTHARD, A., Naae, D., SPILKER, K., HICKS, J., Bhattacharya, S., and SPINDLER, P., Solvent-enhanced biomass liquefaction, Catchlight Energy Llc, Editor. 2012, Google Patents.

5. Heitz, M., Brown, A., and Chornet, E., Solvent effects on liquefaction: solubilization profiles of a Canadian prototype wood, populus deltoides, in the presence of different solvents. Canadian Journal of Chemical Engineering, 1994. 72(6): p. 1021-1027.

6. van Rossum, G., Zhao, W., Castellvi Barnes, M., Lange, J.-P., and Kersten, S.R.A., Liquefaction of Lignocellulosic Biomass: Solvent, Process Parameter, and Recycle Oil Screening. ChemSusChem, 2013: p. $253-259$.

7. Castellví Barnés, M., Lange, J.P., van Rossum, G., and Kersten, S.R.A., A new approach for bio-oil characterization based on gel permeation chromatography preparative fractionation. Journal of Analytical and Applied Pyrolysis, 2015. 113: p. 444-453.

8. Kumar, S., Lange, J.-P., Van Rossum, G., and Kersten, S.R.A., Liquefaction of Lignocellulose: Process Parameter Study To Minimize Heavy Ends. Industrial \& Engineering Chemistry Research, 2014. 53(29): p. 11668-11676.

9. Akhtar, J. and Amin, N.A.S., A review on process conditions for optimum bio-oil yield in hydrothermal liquefaction of biomass. Renewable and Sustainable Energy Reviews, 2011. 15(3): p. 1615-1624.

10. Lange, J.P., Lignocellulose conversion: An introduction to chemistry, process and economics. Biofuels, Bioproducts and Biorefining, 2007. 1(1): p. 39-48.

11. Hildebrand, J. and Scott, R.L., The Solubility of Nonelectrolytes. 3rd ed, ed. A.C. Society. 1950, New York: Reinhold. 
12. Darby, J.R., Touchette, N.W., and Sears, K., Dielectric constants of plasticizers as predictors of compatibility with polyvinyl chloride. Polymer Engineering \& Science, 1967. 7(4): p. 295309.

13. Hansen, C.M., Hansen solubility parameters: A user's handbook. 2000, United States of America: CRC Press LLC. 
$\bar{\infty}$

$\frac{\Omega}{0}$

兴

。

龺

7

กั

$\bar{z}$

三

on

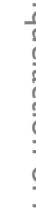

믐

D 


\section{Chapter 7}

Summary, conclusions and recommendations 


\section{Summary, conclusions and recommendations}

The work presented in this thesis studied the direct liquefaction of lignocellulosic biomass for the production of bio-crude, focusing on the analysis of the liquefaction products, the chemistry of the process and the effect of the liquefaction medium.

Initial boundary conditions were defined to maintain low process costs, namely, relatively low temperature $\left(\sim 300^{\circ} \mathrm{C}\right)$ and pressure $(<60 \mathrm{bar})$. A critical parameter was also the liquefaction medium, which needed to be cheap or produced within the process, and either easily recoverable or suitable for co-processing with the biocrude.

In chapter 3 we performed a screening of process parameters and a few liquefaction media, and a set of experiments that simulated a continuous process with recycling of the bio-crude. Results showed that the use of phenolic solvents, small concentrations of water, temperatures of $300-320^{\circ} \mathrm{C}$ and reaction times of 30 minutes led to the highest oil yields ( $\sim 90 \mathrm{C} \%$ ). The liquefaction efficiency of the phenolic solvents suggested that bio-crude could also be a good liquefaction medium. Indeed, recycling experiments showed high bio-crude yields. However, they also suffered from a rapid build-up of heavy species and a fast increase of the bio-crude viscosity.

In chapter 4, GPC preparative fractionation was successfully used for bio-crude isolation from the liquefaction medium and to separate this bio-crude into various fractions based on Mw. Results showed that bio-crude has an atomic and chemical composition similar to lignin and is relatively homogeneous along the whole range of Mw. The bio-crude composition was compared to hydrothermal liquefaction and fast pyrolysis oils, and its quality with the initial biomass and conventional (fossil) fuels.

In chapter 5, the liquefaction of wood's main components provided a better understanding of the chemistry of liquefaction and the origin of the undesired liquefaction products. Char and gas have mainly carbohydrate origin, while the heavy fraction of the bio-crude comes mainly from lignin. A reaction scheme for the 
conversion of carbohydrates and lignin into the various liquefaction products was suggested. Possible reaction mechanisms for the conversion of the wood constituents into bio-crude were also discussed (e.g. conversion of carbohydrates into phenolic and aliphatic species).

Finally, chapter 6 showed the effect of the liquefaction medium on the product distribution and liquefaction kinetics. Liquefaction media with a good interaction (in terms of solubility parameters) with the biomass and its immediate decomposition products led to low char yields. Media containing alcohol functionalities exhibited low wood conversion rates, whereas the addition of water led to higher wood conversion rates.

Other conclusions and recommendations that can be extracted from this thesis are:

- Bio-crude was composed of two phases, a liquid phase named bio-oil and a paste-like phase named bio-tar. Despite their structural and compositional differences, the main wood components (i.e. cellulose, hemicellulose and lignin) produced all very similar bio-oils. Bigger differences were observed between their tars, which still contained partially unconverted biomass.

Characterization results showed that carbohydrates convert into both furanic and phenolic aromatics. However, these two types of component could only be differentiated by GC-MS, which can only analyse the volatile fraction of the biocrude. For a more precise characterization, we therefore suggest the use of techniques that can analyse the whole $\mathrm{Mw}$ range of the bio-crude and also differentiate between furanic and phenolic species, for instance 2D-NMR as applied recently for solid residues [1, 2]. In addition, it is also worth studying the reaction mechanisms that lead to the formation of phenols, as most of the mechanisms presented in literature have not been experimentally proven or have been performed under pyrolytic conditions and therefore might differ under liquefaction conditions. 
Results also showed that biomass deoxygenation occurs mainly via dehydration and, to a minor extent, via decarboxylation and decarbonylation reactions. Higher water content in the medium led to more extensive dehydration and therefore to bio-crudes with lower oxygen content. Further work could focus on the enhancement of the decarboxylation reactions during liquefaction to maximize $\mathrm{O}$ removal and minimize $\mathrm{H}$ loss, thus increasing the $\mathrm{H} / \mathrm{C}_{\text {eff. }}$

- The high content of heavy species in the bio-crude is a major drawback of the liquefaction process. Liquefaction of wood's main components showed that heavy bio-crude has mainly lignin origin and is formed through condensation reactions occurring in parallel with lignin decomposition. Further research should be performed to determine whether and under which conditions the formation of heavies can be prevented. The absence of heavy species in the liquefaction product would allow the direct recycling of the bio-crude as liquefaction medium without the need of an intermediate upgrading or fractionation step.

- The quality of the bio-crude was higher than the initial biomass in terms of oxygen content, $\mathrm{H} / \mathrm{C}$ effective ratio and $\mathrm{HHV}$, but lower than the conventional fuels. Furthermore, bio-crude contained considerable amounts of heavy species that increase its coking tendency and can cause pipe blockage. These results show that bio-crude upgrading is essential before its introduction into the existing transport infrastructure. Future work should focus on the upgrading of the bio-crude to reduce its oxygen content and Mw.

- Char has mainly carbohydrate origin. Char formation during the liquefaction of wood or crystalline cellulose leads to a product that maintains the fibrous structure of the initial biomass. In contrast, the char obtained in glucose or amylopectin liquefaction presents an amorphous glass-like structure and is formed through dehydration, cross-linking and condensation of reaction intermediates. In all cases, char yield is determined by the capacity of the 
liquefaction medium to dissolve the carbohydrates and their initial decay products, as it could be observed from the effect of the medium solubility parameters or the carbohydrate concentration on the char yields. No char formation was caused by the condensation of the final liquefaction products according to the stable char and bio-crude yields observed at extended reaction times.

- The liquefaction medium proved to have an important effect on bio-crude composition and especially on the bio-crude and char yields. Results revealed that a good interaction (in terms of Hildebrand parameter or Hansen distance, $\mathrm{Ra}$ ) of the medium with the biomass or its initial decay products leads to low char and high bio-crude yields. Results also suggested that bio-crude formation occurs in the biomass-solvent interphase as parallel reaction to charring. Further research is required to determine whether the wood conversion occurs only at the surface of the cell wall or if solvent swelling allows wood conversion deep inside the cell wall.

Results showed that alcohol groups in the liquefaction medium decrease the wood conversion rate. In addition, nearly all experiments performed in the presence of alcohols led to $0 \mathrm{C} \%$ char yield. Unfortunately, the incomplete wood conversions obtained for most of those experiments impeded to determine whether, at full wood conversion, all alcohols lead to $0 \mathrm{C} \%$ char or if their char yield is only determined by solubility parameters of the liquefaction medium, as it occurs in the other types of media. In addition, solvents containing alcohol groups tend to react and incorporate into the bio-crude. This could be related to the effect that alcohols have on the wood conversion rate or on char formation. The effect of the alcohol groups during liquefaction definitely calls for further study, and further work should include experiments at complete wood conversion and an analysis of the interactions of alcohols with the biomass and reaction intermediates. 
The addition of small amounts of water into the liquefaction medium resulted in faster wood conversion rates and lower char yields. This water could either be introduced as an external solvent or (preferably) as biomass moisture.

Based on the characterization results, bio-crude has a chemical composition similar to that of lignin and therefore it would be expected to have a relatively good interaction with the biomass and lead to low char yields when used as liquefaction medium. Indeed, recycling experiments performed in chapter 3 presented low char yields $(<1 \mathrm{wt} \%)$. However, they also suffered from a fast accumulation of heavy species due to condensation of the heavies in the (biocrude) medium with the reaction intermediates.

- $\quad$ GPC preparative fractionation is a promising technique for both isolation and fractionation of the bio-crude. Bio-crude isolation is essential to allow a proper characterization of liquefaction bio-crude when other techniques (e.g. distillation or extraction) cannot be used. Bio-crude fractionation is convenient when the components of a specific molecular range (e.g. heavy species) need to be characterized separately. However, for certain experiments a small fraction of the bio-crude was retained in the separation column. In addition, a cleaner separation of the various bio-crude fractions would be of an advantage when narrow $\mathrm{Mw}$ ranges need to be isolated. Thus, further research should be performed to improve the technique. For instance, new mobile and stationary phases should be tested to assure a proper dissolution of the bio-crude and to prevent retention of the bio-crude in the chromatographic column. In addition, the use of a more appropriate solvent and packing, as well as the use of various columns in parallel would allow a better separation of the bio-crude fractions.

Up to date, lignocellulose liquefaction has not yet reached a commercial state due to the generally extreme process conditions, the accumulation of heavy species and solids in the bio-crude, and the loss of light species in the aqueous phase. Most of 
these obstacles have been addressed in the current work, and results showed that lignocellulose liquefaction can be performed at relatively mild conditions, with no water or small water concentrations, and still lead to high bio-crude yields. The results reported in this thesis and in the thesis of S. Kumar [3] showed that bio-crude can be obtained with high oil yields, moderate oxygen content and at competitive prices $(\sim 60 / \mathrm{bbl})$ when using a promising process concept in which the light biocrude is separated and recycled as liquefaction medium. However, the presence of heavy species decreases the bio-crude quality and hinders its upgrading. This issue could not be solved in the current work. Thus, future research should focus on solving these challenges to be able to develop a commercially feasible process. 


\section{References}

1. van Zandvoort, I., E.J. Koers, M. Weingarth, P.C.A. Bruijnincx, M. Baldus, and B.M. Weckhuysen, Structural characterization of 13C-enriched humins and alkali-treated 13C humins by 2D solid-state NMR. Green Chemistry, 2015. 17(8): p. 4383-4392.

2. Johnson, R.L., J.M. Anderson, B.H. Shanks, X. Fang, M. Hong, and K. Schmidt-Rohr, Spectrally edited 2D 13C13C NMR spectra without diagonal ridge for characterizing 13Cenriched low-temperature carbon materials. Journal of Magnetic Resonance, 2013. 234: p. 112-124.

3. Kumar, S., Direct Liquefaction of Lignocellulose: Exploration, Design and Evaluation of Conceptual Processes, in Sustainable Process Technology. 2015, University of Twente: Netherlands. p. 166. 


\section{Samenvatting}

Het onderzoek dat in deze thesis gepresenteerd wordt, betreft een studie naar directe liquefactie van lignocellulose biomassa voor de productie van bio-crude. Hierbij ligt de focus op de analyse van de liquefactieproducten, de chemie van het proces en het effect van het liquefactiemedium.

Voor het handhaven van lage proceskosten zijn verschillende beginvoorwaarden voor het proces gedefinieerd, namelijk een relatief lage temperatuur $\left(\sim 300^{\circ} \mathrm{C}\right)$ en druk ( $<60$ bar). Daarnaast was een kritische parameter het liquefactiemedium, welke goedkoop moest zijn of binnen het proces geproduceerd moest kunnen worden. Daarnaast moest het medium ofwel gemakkelijk te regenereren zijn, ofwel geschikt zijn voor coproductie met de bio-crude.

Hoofdstuk 3 beschrijft een screening van procesparameters en een aantal liquefactiemedia. Ook wordt een set experimenten beschreven die een continu proces simuleerden met recycle van de bio-crude. De resultaten toonden aan dat het gebruik van phenolische oplosmiddelen, kleine concentraties van water, temperaturen van $300-320^{\circ} \mathrm{C}$ en reactietijden van 30 minuten, tot de hoogste opbrengst van olie leidden ( $90 \mathrm{C} \%)$. De efficiëntie van de liquefactie met de phenolische oplosmiddelen suggereerde dat bio-crude ook een goed liquefactiemedium zou kunnen zijn. Experimenten waarbij bio-crude gerecycled werd toonden inderdaad hoge opbrengst van bio-crude. In deze experimenten vond echter ook een snelle opbouw van zware componenten plaats en een snelle toename van de viscositeit van de bio-crude.

In hoofdstuk 4 is preparatieve fractionering met GPC succesvol gebruikt voor het isoleren van bio-crude van het liquefactiemedium en om deze bio-crude te scheiden in verschillende fracties gebaseerd op Mw. De resultaten toonden dat bio-crude een atomaire en chemische samenstelling heeft die vergelijkbaar is met lignine en daarnaast relatief homogeen is over het gehele bereik van het Mw. De bio-crude 
samenstelling is vergeleken met oliën van hydrothermale liquefactie en fast pyrolysis. Daarnaast is de kwaliteit ervan vergeleken met de initiële biomassa en conventionele (fossiele) brandstoffen.

In hoofdstuk 5 verschafte de liquefactie van de hoofdbestanddelen van hout een beter begrip van de chemie van liquefactie en de oorsprong van de ongewenste liquefactieproducten. Kool en gas vinden voornamelijk hun origine in de koolhydraten, terwijl de zware fractie van de bio-crude voornamelijk voortkomt uit lignine. Er werd een reactieschema voorgesteld voor de conversie van koolhydraten en lignine in de verscheidene liquefactieproducten. Mogelijke reactiemechanismen voor de conversie van bestanddelen van hout in bio-crude werden ook bediscussieerd (bijv. conversie van koolhydraten in phenolische en alifatische componenten).

Tot slot toonde hoofdstuk 6 het effect van het liquefactiemedium op de productverdeling en de kinetiek van de liquefactie. Liquefactiemedia met een goede interactie met de biomassa (in termen van oplosbaarheidparameters) en met de directe decompositieproducten leidden tot lage koolopbrengsten. Media die alcoholfunctionaliteiten bevatten, toonden lage conversiesnelheden van hout, terwijl toevoegen van water leidde tot hogere conversiesnelheden van hout.

Op dit moment heeft de liquefactie van lignocellulose nog niet het punt van commerciële toepassing bereikt vanwege de over het algemeen extreme procescondities, de accumulatie van zware componenten en vaste stoffen in de biocrude en het verlies van lichte componenten in de waterige fase. De meeste van deze hindernissen zijn aan de orde geweest in dit onderzoek en de resultaten gaven aan dat de liquefactie van lignocellulose uitgevoerd kan worden bij relatief milde condities, zonder water of met kleine waterconcentraties, en dan nog steeds resulteren in hoge opbrengst van bio-crude. De resultaten die gerapporteerd zijn in deze thesis en in de thesis van S. Kumar [1] toonden dat bio-crude kan worden verkregen met hoge opbrengst van olie, matig zuurstofgehalte en tegen concurrerende prijzen $(\sim 60 / \mathrm{bbl})$ indien een veelbelovend procesconcept wordt 
gebruikt. Hierin wordt de lichte bio-crude afgescheiden en gerecycled als liquefactiemedium. De aanwezigheid van zware componenten verlaagt echter de kwaliteit van de bio-crude en belemmert de opwerking ervan. Dit probleem kon niet worden opgelost in dit werk. Om die reden zou toekomstig onderzoek moeten focussen op het oplossen van deze uitdagingen om een commercieel uitvoerbaar proces te ontwikkelen.

\section{Referenties}

1. Kumar, S., Direct Liquefaction of Lignocellulose: Exploration, Design and Evaluation of Conceptual Processes, in Sustainable Process Technology. 2015, University of Twente: Netherlands. p. 166. 
$\frac{\bar{N}}{N}$ 


\section{Resum}

Aquesta tesis tracta la liqüefacció directa de lignocel-lulosa per a la producció de biocombustibles liquids. La feina realitzada es centra en l'anàlisis dels productes de la liqüefacció, en la química del procés i en l'efecte del medi de reacció.

Per tal de mantenir baixos els costos del procés, es van definir unes condicions inicials de temperatura i pressió relativament baixes (aproximadament $300^{\circ} \mathrm{C}$ i menys de 60 bar respectivament). El medi de liqüefacció o de reacció utilitzat és també un factor crític, i per tant, ha de ser barat o produït durant la liqüefacció. També ha de ser fàcil de recuperar després de la liqüefacció, o s’ha de poder processar juntament amb el bio-oli en els posteriors processos de refinatge.

En el capítol 3 s'examina l'efecte de diversos paràmetres del procés, l'ús de diversos medis de reacció $i$, finalment, es realitza una sèrie d'experiments que simulen un procés de liqüefacció en continu amb reciclatge del bio-oli produït com a medi de reacció. Els resultats obtinguts demostren que l'ús de fenols com a medi de reacció, baixes concentracions d'aigua, temperatures de $300-320^{\circ} \mathrm{C}$ i temps de reacció de 30 minuts dónen els rendiments de bio-oli més elevats ( $90 \mathrm{C} \%)$. L'eficàcia dels fenols com a medi de reacció suggereix que el bio-oli també pot ser un bon medi de reacció, suposició que és confirmada pels alts rendiments de bio-oli obtinguts en els experiments amb reciclatge. Tot i així, aquests experiments també pateixen una ràpida acumulació de compostos pesants i un ràpid augment de la viscositat del biooli.

En el capítol 4, el fraccionament preparatiu del bio-oli es va dur a terme per tal de separar el bio-oli del medi de reacció i per dividir el bio-oli en varies fraccions de diferents pesos moleculars. Els anàlisis de les fraccions obtingudes van demostrar que el bio-oli és relativament homogèni a través de tot el rang de pesos moleculars, i que té una composició atòmica i química semblant a la de la lignina. La composició del bio-oli es va comparar amb les composicions dels olis obtinguts mitjançant 
liqüefacció hidrotèrmica i piròlisis ràpida. La qualitat del bio-oli es va comparar amb la biomassa inicial i amb combustibles (fòssils) tradicionals.

En el capítol 5, la liqüefacció dels tres constituents principals de la lignocelulosa (cel-lulosa, hemicel-lulosa i lignina) proporciona un millor coneixement de la química del procés i de l'orígen dels productes no desitjats. El residu (sòlid) carbonós i el gas es formen principalment a partir dels carbohidrats, mentres que la fracció pesada del bio-oli s'origina majoritàriament a partir de la lignina. En aquest capítol es proposa un esquema de reacció per a la conversió de carbohidrats i lignina en els diversos productes de reacció, i s'analitzen els possibles mecanismes de reacció per a la conversió dels constituents de la fusta en bio-oli (per exemple, per a la conversió de carbohidrats en compostos fenòlics o alifàtics).

Finalment, el capítol 6 mostra l'efecte del medi de reacció sobre la distribució de productes de liqüefacció i la cinètica de reacció. Els medis de liqüefacció que presenten una bona interacció (en termes de paràmetres de solubilitat) amb la biomassa o els seus productes de descomposició més immediats, condueixen als rendiments de residu carbonós més baixos. Per altra banda, els medis de reacció que contenen grups alcohol exhibeixen velocitats de conversió més baixes, mentres que l'addició d'aigua resulta en velocitats de conversió més altes.

Actualment, la liqüefacció de biomassa no ha assolit encara un nivell comercial degut a les condicions generalment extremes del procés, a l'acumulació d'espècies pesants i solids en el bio-oli $i$ a la pèrdua de compostos lleugers en les fases aquoses. La majoria d'aquests punts han estat abordats en aquesta tesis. Els resultats obtinguts han demostrat que la liqüefacció de lignocel-lulosa es pot dur a terme en condicions relativament suaus i sense o amb baixes concentracions d'aigua, i tot $\mathrm{i}$ així donar alts rediments de bio-oli. Els resultats presentats en aquesta tesis i en la tesis de S. Kumar [1] demostren que es poden obtenir alts rendiments de bio-oli amb un contingut moderat d'oxígen i a preus competitius $(\sim 60 / \mathrm{bbl})$ si s'utilitza un procés prometedor en el qual només la fracció lleugera del bio-oli és reciclada com a medi de reacció. Tot i així, la presència de compostos pesants disminueix la qualitat del 
bio-oli i dificulta el seu refinatge. Desafortunadament, aquest problema encara no s'ha pogut resoldre i per tant, futures investigacions s'haurien de centrar en millorar aquests aspectes per tal de desenvolupar un procés que sigui econòmicament viable.

\section{Referències}

1. Kumar, S., Direct Liquefaction of Lignocellulose: Exploration, Design and Evaluation of Conceptual Processes, in Sustainable Process Technology. 2015, University of Twente: Netherlands. p. 166. 
$\frac{\bar{\sigma}}{\text { ज }}$ 


\section{List of symbols}

ATR Attenuated total reflection

$\mathrm{C} \% \quad$ Carbon percentage

CGO Cracked Gas Oil

$\bigoplus_{M} \quad$ Dispersity

DMSO Deuterated methyl sulfoxide

DTGS deuterated triglycine sulfate detector

EA Elemental analysis

Ea Activation energy

FCC Fluid Catalytic Cracking

FTIR Fourier Transform Infrared Spectroscopy

GC Gas chromatography

GC-MS Gas chromatography-Mass spectrometry

GL Liquefaction in guaiacol

GPC Gel permeation chromatography

GWL Liquefaction in guaiacol:water mixture (1:1)

$\mathrm{H} / \mathrm{C}_{\text {eff }} \quad$ Effective hydrogen to carbon ratio

$\mathrm{HHV} \quad$ Higher heating value

HR-SEM High resolution-Scanning electron microscopy

k Rate constant

$\mathrm{k}_{0} \quad$ Pre-exponential factor

KFT Karl-Fischer titration

LCO Light Cycle Oil

$\mathrm{m} / \mathrm{z} \quad$ Mass to charge ratio

MCRT Micro carbon residue test

Mw Molecular weight 


\begin{tabular}{ll} 
MwGPC & Apparent molecular weight (measured with GPC) \\
NMR & Nuclear magnetic resonance \\
$\mathrm{R}$ & Ideal gas constant \\
$\mathrm{Ra}$ & Hansen distance or solubility radius \\
$\mathrm{RID}$ & Refractive index detector \\
$\mathrm{st}$. & Stretching \\
$\mathrm{t}$ & time \\
$\mathrm{T}$ & Temperature \\
$\mathrm{THF}$ & Tetrahydrofuran \\
$\mathrm{VGO}$ & Vacuum Gas Oil \\
$\mathrm{Vm}$ & Molar volume \\
$\mathrm{VR}$ & Vacuum residue \\
$\mathrm{WL}$ & Liquefaction in water \\
$\mathrm{wt} \%$ & weight percentage \\
$\delta$ & Hildebrand solubility parameter \\
$\delta \mathrm{D}$ & Dispersion partial solubility parameter \\
$\delta \mathrm{H}$ & H-bonding partial solubility parameter \\
$\delta \mathrm{P}$ & Polarity partial solubility parameter \\
$\Delta \mathrm{H}_{\mathrm{v}}$ & Enthalpy of vaporization \\
$\varepsilon$ & Dielectric constant \\
$\phi$ & Volume fraction \\
\hline
\end{tabular}




\section{List of publications}

- $\quad$ van Rossum, G.; Zhao, W.; Castellvi Barnes, M.; Lange, J.-P. and Kersten, S. R. A.; Liquefaction of Lignocellulosic Biomass: Solvent, Process Parameter, and Recycle Oil Screening. ChemSusChem, 2013, 7(1): p. 253-259.

- Castellví Barnés, M.; Lange, J.-P.; van Rossum, G. and Kersten, S. R. A.; A new approach for bio-oil characterization based on gel permeation chromatography preparative fractionation. Journal of Analytical and Applied Pyrolysis, 2015, 113: 444-453.

- Castellví Barnés, M.; de Visser, M. M.; Lange, J.-P.; van Rossum, G. and Kersten, S.R.A.; Chemistry of the Liquefaction of wood and its model components. Submitted to ACS Sustainable Chemistry \& Engineering.

\section{In preparation:}

- $\quad$ Castellví Barnés, M.; Oltvoort, E. J.; Lange, J.-P. and Kersten, S.R.A.; Effect of the reaction medium during liquefaction of lignocellulose.

\section{Patents}

- $\quad$ Castellví Barnés M.; Kersten, S. R. A.; Kumar, S.; Lange, J.-P.; Zhao, W.; Process for conversion of a cellulosic material. US 20130247449 A1, May 2013.

\section{Other publications}

- Oral presentation and poster at the Renewable Resources and Biorefineries conference (RRB10). Presentation and poster title: A new approach for bio-oil characterization based on GPC preparative fractionation, 2014. 
- Oral presentation at the Netherlands Process Technology Symposium (NPS14). Presentation title: A new approach for bio-oil characterization based on GPC preparative fractionation, 2014. 


\section{A. Tables (Chapter 3)}

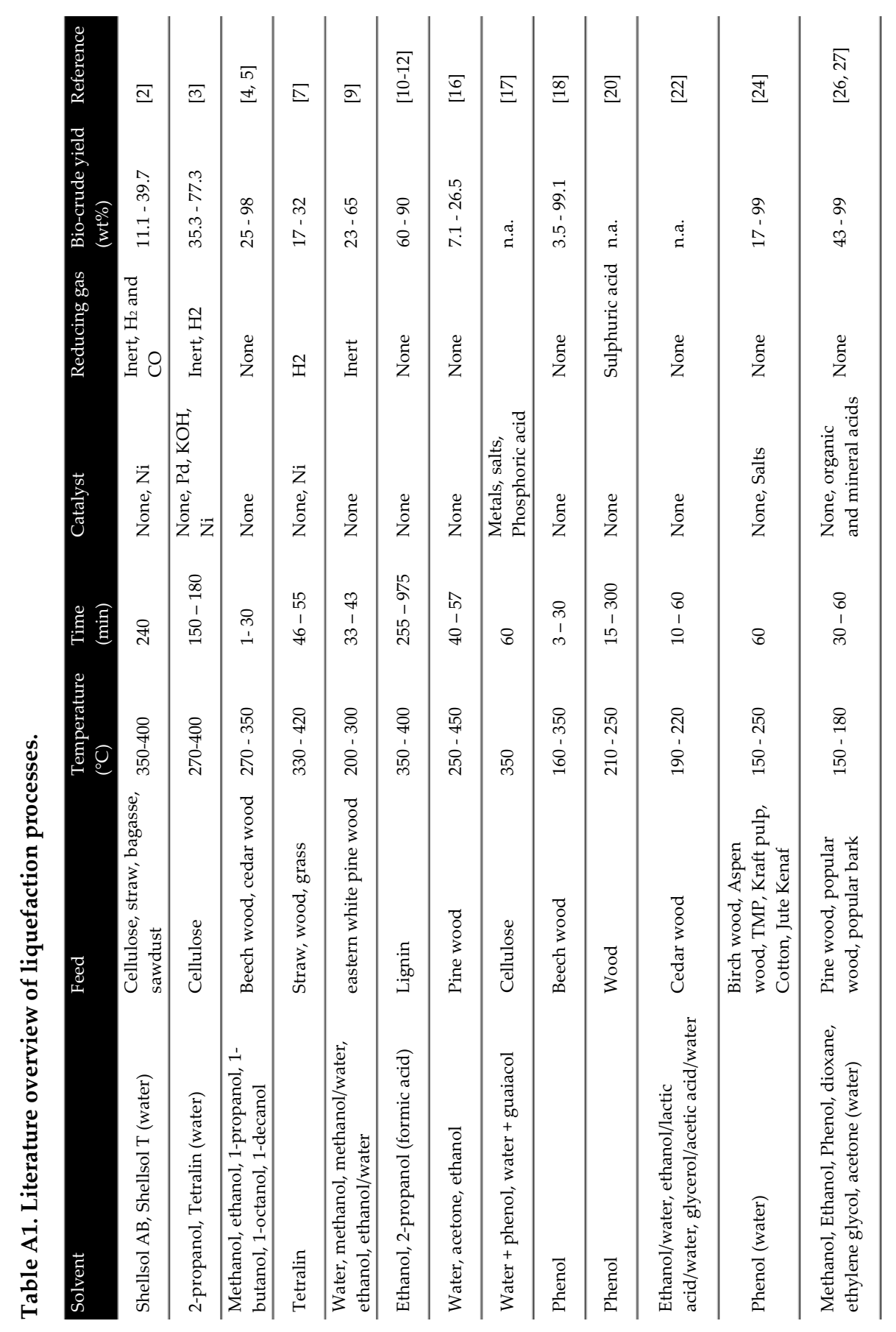




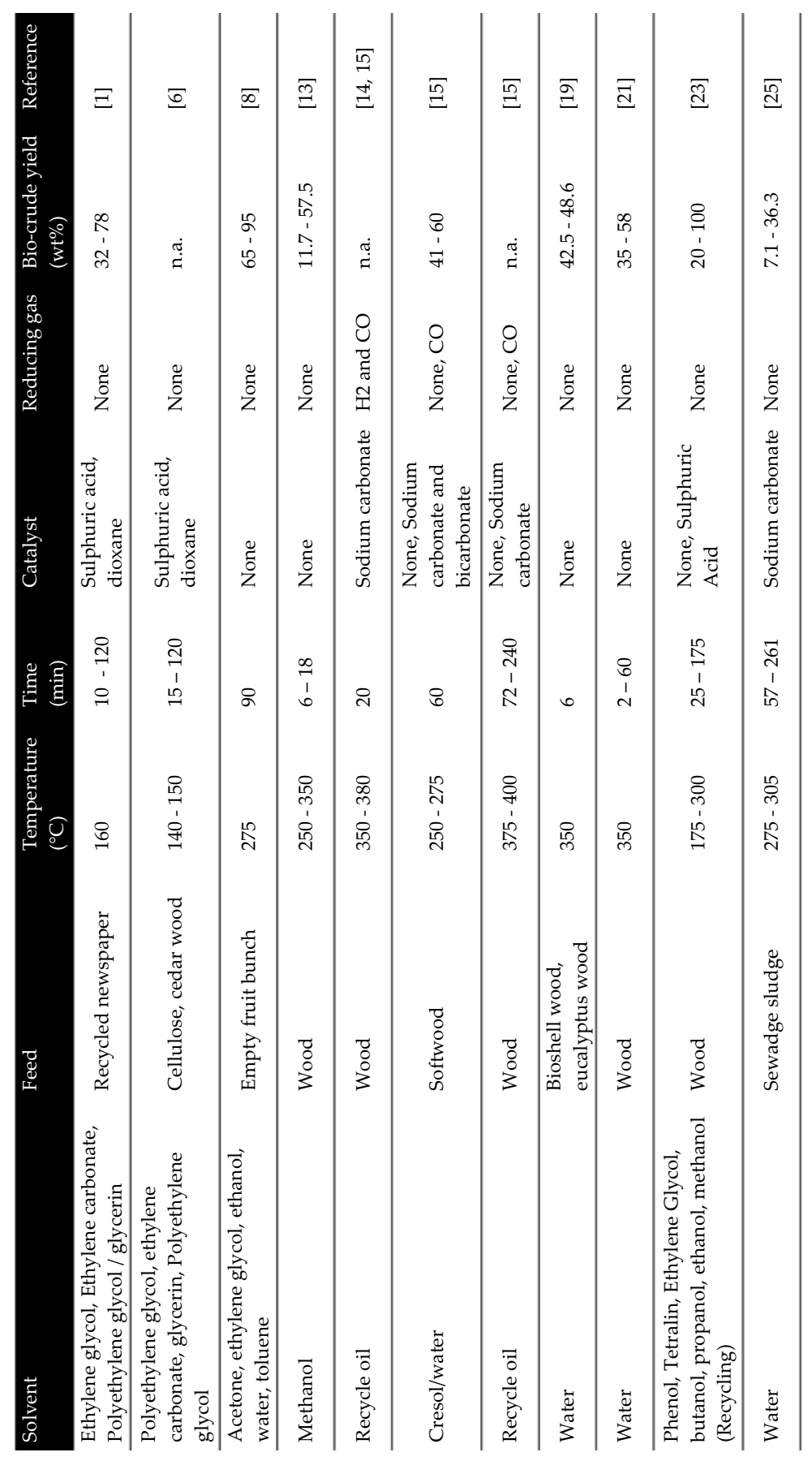


Table A2. Detailed experimental data.

\begin{tabular}{|c|c|c|c|c|c|c|c|c|c|c|c|c|c|}
\hline 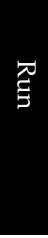 & 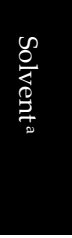 & $\begin{array}{l}\sum \\
8 \\
0\end{array}$ & 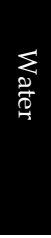 & $\begin{array}{l}\infty \\
\frac{0}{4} \\
\stackrel{D}{ٍ}\end{array}$ & 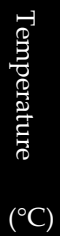 & 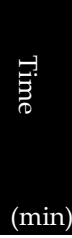 & $\begin{array}{l}\text { D } \\
\bar{D} \\
0 \\
0 \\
0 \\
0 \\
0\end{array}$ & $\underset{\infty}{\infty}$ & 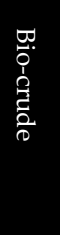 & 怘 & $\underset{\nexists}{\infty}$ & $\begin{array}{l}\text { ్․ } \\
0 \\
1 \\
0 \\
0 \\
0 \\
0 \\
0\end{array}$ & $\begin{array}{l}\text { no } \\
\frac{0}{2}\end{array}$ \\
\hline 1 & PY & 100 & 0 & 0 & 317 & 30 & 51 & 7.0 & 13.9 & 79.1 & 10.7 & 37.8 & 51.5 \\
\hline 2 & $\mathrm{HO}$ & 10 & 90 & 0 & 302 & 20 & 108 & 1.2 & 73.5 & 25.3 & 1.9 & $81.4^{\mathrm{e}}$ & 16.7 \\
\hline 3 & GC & 10 & 10 & 80 & 305 & 30 & 60 & 4.7 & 93.4 & 2.0 & 7.1 & 90.5 & 2.4 \\
\hline 4 & GC & 10 & 0 & 90 & 305 & 30 & 29 & 3.7 & 74.4 & 22.0 & 5.2 & 70.4 & 24.4 \\
\hline 5 & HA & 10 & 10 & 80 & 303 & 30 & 71 & 2.4 & 87.8 & 9.8 & 3.4 & 89.6 & 7.1 \\
\hline 6 & HA & 10 & 0 & 90 & 304 & 30 & 39 & 2.6 & 60.4 & 37.0 & 3.6 & 67.4 & 29.1 \\
\hline 7 & UD & 10 & 10 & 80 & 305 & 30 & 121 & 2.3 & 46.8 & 51.0 & 3.7 & 61.2 & 35.1 \\
\hline 8 & UD & 10 & 0 & 90 & 306 & 30 & 56 & 3.0 & 40.0 & 57.0 & 4.5 & 55.6 & 40.0 \\
\hline 9 & $\mathrm{GC}^{\mathrm{b}}$ & 10 & 0 & 90 & 305 & 30 & 32 & 3.1 & 74.9 & 22.1 & 4.3 & 71.5 & 24.2 \\
\hline 10 & $\mathrm{GC}^{b}$ & 10 & 0 & 90 & 305 & 30 & 33 & 5.0 & 74.2 & 20.8 & 7.1 & 70.5 & 22.4 \\
\hline 11 & GC & 10 & 5 & 85 & 300 & 30 & 45 & 4.2 & 87.2 & 8.6 & 6.3 & 83.9 & 9.8 \\
\hline 12 & GC & 10 & 5 & 85 & 300 & 30 & 47 & 5.2 & 86.1 & 8.7 & 7.3 & 83.0 & 9.6 \\
\hline 13 & $\mathrm{GC}^{\mathrm{b}}$ & 10 & 10 & 80 & 306 & 30 & 57 & 4.7 & 93.4 & 1.9 & 7.2 & 90.3 & 2.5 \\
\hline 14 & $\mathrm{GC}^{b}$ & 10 & 10 & 80 & 302 & 30 & 58 & 5.6 & 92.5 & 1.9 & 8.0 & 89.8 & 2.2 \\
\hline 15 & GC & 10 & 45 & 45 & 300 & 30 & 97 & 5.9 & 94.0 & 0.1 & 9.4 & 90.5 & 0.1 \\
\hline 16 & GC & 10 & 80 & 10 & 300 & 30 & 108 & 6.5 & 89.1 & 4.4 & 10.5 & 86.4 & 3.1 \\
\hline 17 & GC & 5 & 0 & 95 & 304 & 30 & 24 & 2.9 & 65.9 & 31.2 & 3.9 & 61.8 & 34.3 \\
\hline 18 & GC & 8 & 0 & 92 & 304 & 30 & 28 & 3.3 & 69.6 & 27.1 & 4.5 & $\begin{array}{l}65.8 \\
\end{array}$ & 29.7 \\
\hline 19 & GC & 12 & 0 & 88 & 305 & 30 & 37 & 4.0 & 75.7 & 20.3 & 5.5 & 72.3 & 22.2 \\
\hline 20 & GC & 15 & 0 & 85 & 305 & 30 & 42 & 3.9 & 80.4 & 15.7 & 5.6 & 77.1 & 17.3 \\
\hline 21 & $\mathrm{GC}^{b}$ & 15 & 0 & 85 & 303 & 30 & 40 & 4.2 & 76.8 & 19.0 & 5.9 & 73.4 & 20.7 \\
\hline 22 & GC & 10 & 0 & 90 & 304 & 60 & 32 & 5.8 & 89.9 & 4.3 & 8.1 & 87.1 & 4.8 \\
\hline 23 & GC & 10 & 0 & 90 & 306 & 90 & 44 & 6.4 & 92.2 & 1.4 & 8.9 & 89.5 & 1.6 \\
\hline 24 & GC & 10 & 0 & 90 & 307 & 120 & 45 & 6.6 & 92.1 & 1.3 & 9.2 & 89.3 & 1.5 \\
\hline 25 & GC & 10 & 0 & 90 & 265 & 30 & 18 & 0.9 & 27.9 & 71.2 & 1.3 & 27.8 & 70.8 \\
\hline 26 & GC & 10 & 0 & 90 & 286 & 30 & 23 & 1.9 & 51.9 & 46.2 & 2.7 & 48.9 & 48.3 \\
\hline 27 & GC & 10 & 0 & 90 & 323 & 30 & 44 & 6.3 & 89.3 & 4.4 & 8.7 & 86.3 & 4.9 \\
\hline
\end{tabular}

a GC: Guaiacol, HA: Hexanoic acid, UD: $n$-Undecane, PY: no solvent added, HO: very wet wood only

b Duplo experiment

${ }^{\mathrm{c}}$ Maximum reactor pressure (nitrogen, gas produced and vapour pressure)

${ }^{\mathrm{d}}$ Including possible produced water

e Phase separation occurred; the amount given here is the combined yield 
Table A3. Experimental data of refill experiments.

\begin{tabular}{|c|c|c|c|c|c|c|}
\hline Run & & 1 & 2 & 3 & 4 & 5 \\
\hline \multicolumn{7}{|c|}{ Experimental conditions } \\
\hline Wood & {$[\mathrm{wt} \%]$} & 10 & 10 & 11 & 10 & 22 \\
\hline Water & {$[\mathrm{wt} \%]$} & 10 & 10 & 10 & 10 & 10 \\
\hline Solvent & {$[\mathrm{wt} \%]$} & 80 & 80 & 79 & 80 & 68 \\
\hline Temperature & {$\left[{ }^{\circ} \mathrm{C}\right]$} & 307 & 310 & 309 & 308 & 308 \\
\hline Pressure & [bar] & 54 & 70 & 64 & 53 & 57 \\
\hline
\end{tabular}

\section{Product yield}

\begin{tabular}{lllllll} 
Gas & {$[\mathrm{wt} \%]$} & 11.4 & 15.9 & 13.4 & 8.1 & 13.1 \\
\hline Lights & {$[\mathrm{wt} \%]$} & 11.0 & 0.2 & 0.0 & 7.5 & 5.9 \\
\hline Solid & {$[\mathrm{wt} \%]$} & 6.3 & 0.8 & 0.9 & 0.7 & 0.5 \\
\hline Liquid (difference) & {$[\mathrm{wt} \%]$} & 71.3 & 83.2 & 85.7 & 83.6 & 80.5 \\
\hline
\end{tabular}

\section{Liquid product analysis}

\begin{tabular}{lllllll} 
Guaiacol & {$[\mathrm{wt} \%]$} & 76.6 & 71.0 & 62.4 & 54.1 & 37.5 \\
\hline Water & {$[\mathrm{wt} \%]$} & - & - & - & - & 0.5 \\
\hline Viscosity & {$\left[\mathrm{cP}\right.$ at $\left.30^{\circ} \mathrm{C}\right]$} & 8 & 15 & 28 & 64 & 320 \\
\hline
\end{tabular}

\section{Bio-crude analysis (guaiacol and water free)}

\begin{tabular}{lllllll} 
Carbon & {$[\mathrm{wt} \%]$} & - & - & - & - & 61.8 \\
\hline Hydrogen & {$[\mathrm{wt} \%]$} & - & - & - & - & 6.4 \\
\hline Oxygen (difference) & {$[\mathrm{wt} \%]$} & - & - & - & - & 31.8
\end{tabular}

Table A4. Characteristics of the solvents used for biomass liquefaction.

\begin{tabular}{|c|c|c|c|c|c|c|c|}
\hline \multirow{2}{*}{ Solvent } & \multirow{2}{*}{$\begin{array}{l}\text { Density } \\
{[\mathrm{g} / \mathrm{ml}]}\end{array}$} & \multirow{2}{*}{$\begin{array}{l}\mathrm{Tb} \\
{\left[{ }^{\circ} \mathrm{C}\right]}\end{array}$} & \multirow{2}{*}{$\begin{array}{l}\log \left[\mathrm{P}_{\text {ow }}\right] \\
{[-]}\end{array}$} & \multirow{2}{*}{$\begin{array}{l}\text { Hildebrand } \\
{[\mathrm{J} / \mathrm{ml}]^{0.5}}\end{array}$} & \multicolumn{3}{|c|}{ Hansen $[\mathrm{J} / \mathrm{ml}]^{0.5}$} \\
\hline & & & & & Dispersion & Polar & H-Bonding \\
\hline Water & 1.00 & 100 & $-\infty$ & 47.8 & 15.5 & 16 & 42.3 \\
\hline Guaiacol & 1.12 & 205 & 1.3 & 23.5 & 16.3 & 8.7 & 14.5 \\
\hline Hexanoic acid & 0.92 & 206 & 1.9 & 21.8 & 16.0 & 5.2 & 13.8 \\
\hline Undecane & 0.74 & 196 & 7.2 & 15.9 & 15.9 & 0.0 & 0.0 \\
\hline
\end{tabular}




\section{A.I. References}

1. Shin, H., Kim, C.-J., and Kim, S., Kinetic study of recycled newspaper liquefaction in polyol solvent. Biotechnology and Bioprocess Engineering, 2009. 14(3): p. 349-353.

2. El-Gayar, M.S. and McAuliffe, C.A., Shellsol as a processing liquid in biomass liquefaction. Energy Sources, 1997. 19(7): p. 665-676.

3. Vasilakos, N.P. and Austgen, D.M., Hydrogen-donor solvents in biomass liquefaction. Industrial \& Engineering Chemistry Process Design and Development, 1985. 24(2): p. 304-311.

4. Yamazaki, J., Minami, E., and Saka, S., Liquefaction of beech wood in various supercritical alcohols. Journal of Wood Science, 2006. 52(6): p. 527-532.

5. Minami, E. and Saka, S., Comparison of the decomposition behaviors of hardwood and softwood in supercritical methanol. Journal of Wood Science, 2003. 49(1): p. 0073-0078.

6. Yamada, T., Aratani, M., Kubo, S., and Ono, H., Chemical analysis of the product in acidcatalyzed solvolysis of cellulose using polyethylene glycol and ethylene carbonate. Journal of Wood Science, 2007. 53(6): p. 487-493.

7. Beauchet, R., Pinard, L., Kpogbemabou, D., Laduranty, J., Lemee, L., Lemberton, J.L., Bataille, F., Magnoux, P., Ambles, A., and Barbier, J., Hydroliquefaction of green wastes to produce fuels. Bioresource Technology, 2011. 102(10): p. 6200-6207.

8. Fan, S.P., Zakaria, S., Chia, C.H., Jamaluddin, F., Nabihah, S., Liew, T.K., and Pua, F.L., Comparative studies of products obtained from solvolysis liquefaction of oil palm empty fruit bunch fibres using different solvents. Bioresoure Technology, 2011. 102(3): p. 3521-3526.

9. Cheng, S., D'cruz, I., Wang, M., Leitch, M., and Xu, C., Highly Efficient Liquefaction of Woody Biomass in Hot-Compressed Alcohol-Water Co-solventst. Energy \& Fuels, 2010. 24(9): p. $4659-4667$.

10. Kleinert, M. and Barth, T., Towards a Lignincellulosic Biorefinery: Direct One-Step Conversion of Lignin to Hydrogen-Enriched Biofuel. Energy \& Fuels, 2008. 22(2): p. 13711379.

11. Kleinert, M. and Barth, T., Phenols from Lignin. Chemical Engineering \& Technology, 2008. 31(5): p. 736-745. 
12. Kleinert, M., Gasson, J.R., and Barth, T., Optimizing solvolysis conditions for integrated depolymerisation and hydrodeoxygenation of lignin to produce liquid biofuel. Journal of Analytical and Applied Pyrolysis, 2009. 85(1-2): p. 108-117.

13. Poirier, M.G., Ahmed, A., Grandmaison, J.L., and Kaliaguine, S.C.F., Supercritical gas extraction of wood with methanol in a tubular reactor. Industrial \& Engineering Chemistry Research, 1987. 26(9): p. 1738-1743.

14. Elliott, D.C., Baker, E.G., Beckman, D., Solantausta, Y., Tolenhiemo, V., Gevert, S.B., Hörnell, C., Östman, A., and Kjellström, B., Technoeconomic assessment of direct biomass liquefaction to transportation fuels. Biomass, 1990. 22(1-4): p. 251-269.

15. White, D.H., Wolf, D., and Zhao, Y. Biomass Liquefaction Utilizing Extruder-Feeder Reactor System. in ACS Fuels (Spring meeting). 1987. Denver (CO), USA.

16. Liu, Z. and Zhang, F.-S., Effects of various solvents on the liquefaction of biomass to produce fuels and chemical feedstocks. Energy Conversion and Management, 2008. 49(12): p. 34983504.

17. Miller, I.J. and Fellows, S.K., Catalytic effects during cellulose liquefaction. Fuel, 1985. 64(9): p. 1246-1250.

18. Mishra, G. and Saka, S., Kinetic behavior of liquefaction of Japanese beech in subcritical phenol. Bioresour Technol, 2011. 102(23): p. 10946-10950.

19. Goudriaan, F. and Peferoen, D.G.R., Liquid fuels from biomass via a hydrothermal process. Chemical Engineering Science, 1990. 45(8): p. 2729-2734.

20. Ammar, S., Shakourzadeh, K., Capart, R., and Gelus, M., Simple mathematical model for the solvolysis of cylindrical pine-wood samples. Applied Energy, 1994. 48(2): p. 137-148.

21. Knežević, D., van Swaaij, W., and Kersten, S., Hydrothermal Conversion Of Biomass. II. Conversion Of Wood, Pyrolysis Oil, And Glucose In Hot Compressed Water. Industrial \& Engineering Chemistry Research, 2009. 49(1): p. 104-112.

22. Baba, Y., Tanabe, T., Shirai, N., Watanabe, T., Honda, Y., and Watanabe, T., Pretreatment of Japanese cedar wood by white rot fungi and ethanolysis for bioethanol production. Biomass and Bioenergy, 2011. 35(1): p. 320-324.

23. Rezzoug, S.-A. and Capart, R., Solvolysis and hydrotreatment of wood to provide fuel. Biomass and Bioenergy, 1996. 11(4): p. 343-352. 
24. Maldas, D. and Shiraishi, N., Liquefaction of biomass in the presence of phenol and $\mathrm{H} 2 \mathrm{O}$ using alkalies and salts as the catalyst. Biomass and Bioenergy, 1997. 12(4): p. 273-279.

25. Molton, P.M., Fassbender, A.G., and Brown, M.D., STORS: The Sludge-to-Oil Reactor System. 1986, United States Environmental Protection Agency.

26. Mun, S.P. and Hassan, E.M., Liquefaction of Lignocellulosic Biomass with Mixtures of Ethanol and Small Amounts of Phenol in the Presence of Methanesulfonic Acid Catalyst. J. Ind. Eng. Chem., 2004. 10(5).

27. Mun, S.P. and Hassan, E.M., Liquefaction of Lignocellulosic Biomass with Dioxane/Polar Solvent Mixtures in the Presence of an Acid Catalyst. J. Ind. Eng. Chem., 2004. 10(3): p. 473477. 


$$
\frac{\bar{a}}{\infty}
$$




\section{B. Fractionation}

The procedure used during GPC preparative fractionation can be found in chapter 2 (section 5). After elution through the column, various bio-crude fractions were collected in intervals of one minute and then analysed with a standard analytical GPC (chapter 2, section 7.1) to determine their GPC molecular weight distribution (MwGPC). According to Figure 6 in chapter 4, such fractionation allowed to isolate the light solvent fraction and even separate the heavier bio-crude into several reasonably well-defined fractions.
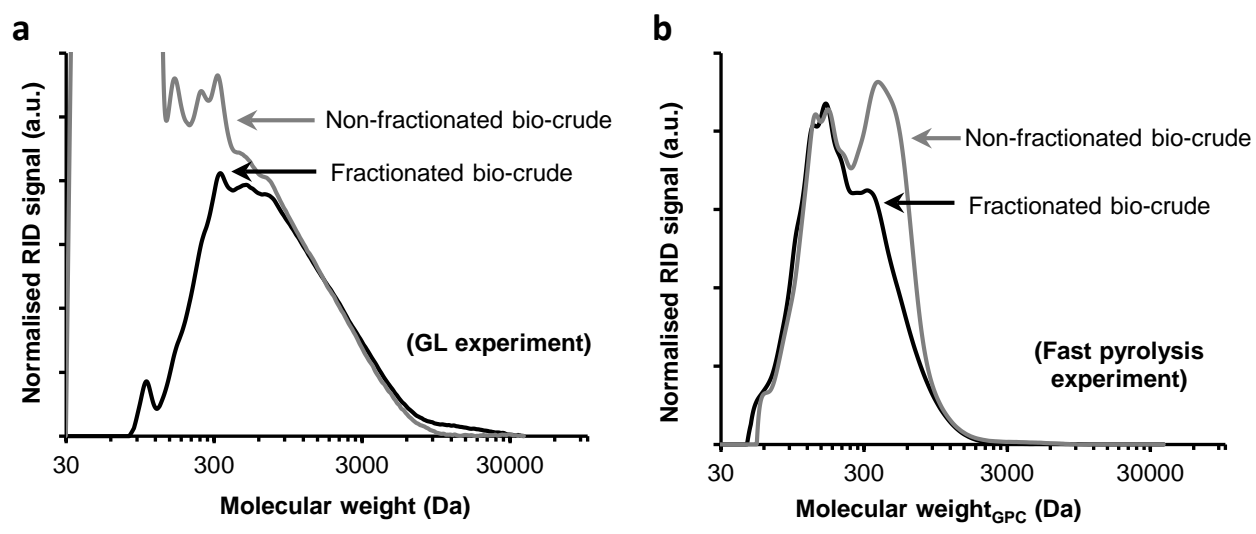

Figure B1. GPC chromatograms of bio-crudes before and after fractionation. GPC of nonisolated and isolated bio-crude obtained after GL (a) and after pyrolysis (b). GL bio-crude obtained at $300^{\circ} \mathrm{C}$ during 30 minutes with $10 \mathrm{wt} \%$ of wood and $90 \mathrm{wt} \%$ of guaiacol. Pyrolysis experiment performed at $300^{\circ} \mathrm{C}$ with residence times of 20-25 minutes for the solid particles and below 2 seconds for the bio-crude.

During the GPC fractionation of the pyrolysis and the hydrothermal liquefaction bio-crudes, a small portion of high molecular weight compounds was lost. Figure B1b shows the MwGPC distribution of the pyrolysis bio-crude before and after fractionation. Approximately $16 \%$ of the compounds with a MwGPC around $500 \mathrm{Da}$ are lost during the fractionation of the bio-crude. This lost fraction has a poor solubility in THF at the high concentrations used during the GPC fractionation but it is soluble at the high dilution used in analytical GPC. Bio-crudes obtained in GL 
and in GWL were completely recovered after fractionation (Figure B1a) due to their higher solubility in THF either because of their chemical composition or because of their higher dilution factor (approximately $10 \%$ and $50 \mathrm{wt} \%$ of bio-crude dissolved in guaiacol) compared to pyrolysis and WL bio-crudes.

Table B1. Elemental composition of the GL and the pyrolysis bio-crudes calculated with and without GPC fractionation.

\begin{tabular}{llllll} 
Experiment & $\begin{array}{l}\mathrm{N} \\
(\mathrm{wt} \%)\end{array}$ & $\begin{array}{l}\mathrm{C} \\
(\mathrm{wt} \%)\end{array}$ & $\begin{array}{l}\mathrm{H} \\
(\mathrm{wt} \%)\end{array}$ & $\begin{array}{l}\mathrm{O}(\mathrm{wt} \%) \\
(\text { by difference) }\end{array}$ & $\begin{array}{l}\mathrm{H} / \mathrm{C} \text { eff. } \\
\text { ratio }\end{array}$ \\
\hline GL with fractionation & 0.03 & 63.03 & 6.00 & 30.94 & 0.03 \\
\hline GL without fractionation & 0.39 & 65.29 & 7.69 & 26.62 & 0.39 \\
\hline Guaiacol & 0 & 67.73 & 6.50 & 25.78 & 0.57 \\
\hline Pyrolysis with fractionation $^{\text {Pyrolysis without fractionation }}{ }^{\mathrm{a}}$ & 0.14 & 56.34 & 6.36 & 37.16 & 0.14 \\
\hline
\end{tabular}

a Elemental composition on water-free basis

The use of the GPC fractionation allowed a decrease of the error associated to the analysis of the bio-crude. For instance, when the elemental compositions of guaiacol and water were subtracted to the elemental composition of the bio-crude and solvent mixture (according to their concentration in the mixture), the errors on the bio-crude's calculated composition were significantly larger than the values obtained after GPC fractionation. Likewise, when guaiacol FTIR spectrum was subtracted to the spectrum of the bio-crude and solvent mixture, a spectrum with negative peaks was obtained. Furthermore, ${ }^{13} \mathrm{C}-\mathrm{NMR}$ analysis of the nonfractionated bio-crude would be impossible, since the low sensitivity of the technique and the low concentration of the bio-crude components would result in a bio-crude signal smaller than the noise of the measurement. This holds especially for the GL, since the liquid mixture (bio-crude with solvent) has a very high guaiacol concentration (around $87 \mathrm{wt} \%$ ). For instance, the non-fractionated GL bio-crude has a higher carbon content and a lower oxygen content than the fractionated bio-crude, approaching the elemental composition of guaiacol (Table B1). These differences in elemental composition also result in a significantly different $\mathrm{H} / \mathrm{C}_{\text {eff }}$ ratio. The elemental compositions of the pyrolysis bio-crude obtained with and without 
fractionation are also included in Table B1 for comparison. The absence of an organic solvent and the not too high water concentration of the pyrolysis bio-crude (27 wt\%) allows a relatively accurate calculation of its elemental composition without using fractionation. 


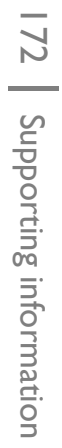




\section{FTIR of model components}

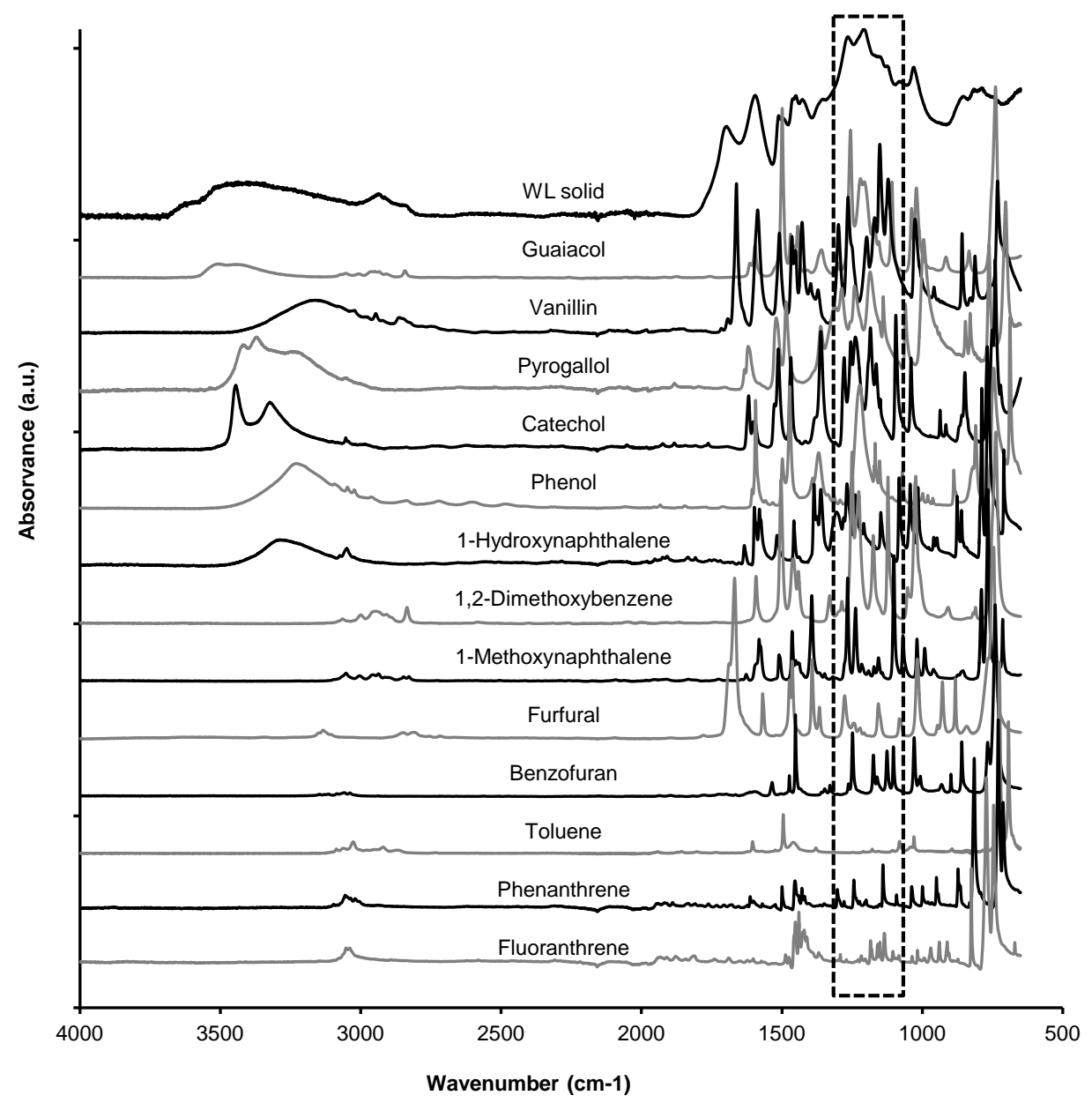

Figure C1. FTIR spectra of model components and the solid residue from WL. WL solid residue obtained at $300^{\circ} \mathrm{C}$ during 30 minutes with $10 \mathrm{wt} \%$ of wood and $90 \mathrm{wt} \%$ of water.

The FTIR of some bio-crude fractions and solid residues present an undefined and broad band with a wavenumber at $1200 \mathrm{~cm}^{-1}$ that is believed to belong to oxygenated aromatic compounds. To verify that this band corresponds to aromatic oxygenates, the FTIR spectra of several model compounds were measured. The results presented in Figure C1 reveal that non-oxygenated aromatics hardly present any band at this 
frequency, and that only aromatics with hydroxyl or ether groups have large bands at $1200 \mathrm{~cm}^{-1}$. Furfural and benzofuran, which contain an oxygen atom inside the aromatic structure, present significantly smaller bands than the molecules with hydroxyl and ether groups. Intriguingly, however, the aromatic oxygenates that show bands around $1200 \mathrm{~cm}^{-1}$ also show significant and sharp bands around $750 \mathrm{~cm}^{-}$ ${ }^{1}$. Likewise, the liquefaction bio-crudes show a significant absorption band around this wavelength, but broader and less intense than the band observed at $1200 \mathrm{~cm}^{-1}$. We presently have no good explanation for this apparent mismatch. 


\section{Tables and figures (Chapter 4)}

Table D2. VR, carbon residue, elemental composition and H/C effective ratio of bio-crudes and model components. Elemental composition of pyrolysis bio-crude was directly measured by EA. Elemental composition of the liquefaction bio-crudes was calculated by numerical addition of their fractions.

\begin{tabular}{llllllll} 
Experiment & $\begin{array}{l}\mathrm{VR} \\
(\mathrm{C} \%)\end{array}$ & $\begin{array}{l}\text { Carbon residue } \\
(\mathrm{wt} \%)\end{array}$ & $\begin{array}{l}\mathrm{N} \\
(\mathrm{wt} \%)\end{array}$ & $\begin{array}{l}\mathrm{C} \\
(\mathrm{wt} \%)\end{array}$ & $\begin{array}{l}\mathrm{H} \\
(\mathrm{wt} \%)\end{array}$ & $\begin{array}{l}\mathrm{O}(\mathrm{wt} \%) \\
(\text { by difference) }\end{array}$ & $\begin{array}{l}\mathrm{H} / \mathrm{C} \text { eff. } \\
\text { ratio }\end{array}$ \\
\hline GL & 35 & 38 & 0.03 & 63.03 & 6.00 & 30.94 & 0.40 \\
\hline GWL & 19 & 29 & 0.02 & 64.20 & 5.90 & 29.88 & 0.40 \\
\hline WL & 21 & 33 & 0.14 & 69.05 & 5.08 & 25.72 & 0.32 \\
\hline Pyrolysis & 2 & 23 & 0.34 & 58.01 & 6.26 & 35.38 & 0.37 \\
\hline Cellulose & - & - & 0 & 44.40 & 6.20 & 49.30 & 0 \\
\hline Lignin & 51 & - & 0.08 & 64.79 & 6.31 & 28.82 & 0.23 \\
\hline Wood & - & - & 0.04 & 46.58 & 6.34 & 46.98 & 0.11 \\
\hline
\end{tabular}

Table D3. Elemental composition and $\mathrm{H} / \mathrm{C}$ effective ratio of bio-crude fractions

\begin{tabular}{llllll} 
Experiment & $\begin{array}{l}\mathrm{N} \\
(\mathrm{wt} \%)\end{array}$ & $\begin{array}{l}\mathrm{C} \\
(\mathrm{wt} \%)\end{array}$ & $\begin{array}{l}\mathrm{H} \\
(\mathrm{wt} \%)\end{array}$ & $\begin{array}{l}\mathrm{O}(\mathrm{wt} \%) \\
(\text { by difference) }\end{array}$ & $\begin{array}{l}\mathrm{H} / \mathrm{C} \text { eff. } \\
\text { ratio }\end{array}$ \\
\hline GL Heavy fr. & 0.05 & 65.42 & 6.22 & 28.31 & 0.48 \\
\hline GL Middle fr. & 0.01 & 60.91 & 5.80 & 33.27 & 0.32 \\
\hline GWL Heavy fr. & 0.01 & 62.84 & 6.06 & 31.08 & 0.41 \\
\hline GWL Middle fr. & 0.02 & 64.94 & 5.81 & 29.24 & 0.39 \\
\hline WL Heavy fr. & 0.05 & 68.79 & 4.83 & 26.32 & 0.26 \\
\hline WL Middle fr. & 0.05 & 68.75 & 4.98 & 26.23 & 0.29 \\
\hline WL Light fr. & 0.90 & 71.26 & 6.56 & 21.28 & 0.65 \\
\hline Pyrolysis Heavy fr. & 0.18 & 61.34 & 6.14 & 32.33 & 0.40 \\
\hline Pyrolysis Middle fr. & 0.24 & 57.11 & 6.37 & 36.28 & 0.38 \\
\hline Pyrolysis Light fr. & 0.04 & 54.9 & 6.4 & 38.7 & 0.33 \\
\hline
\end{tabular}




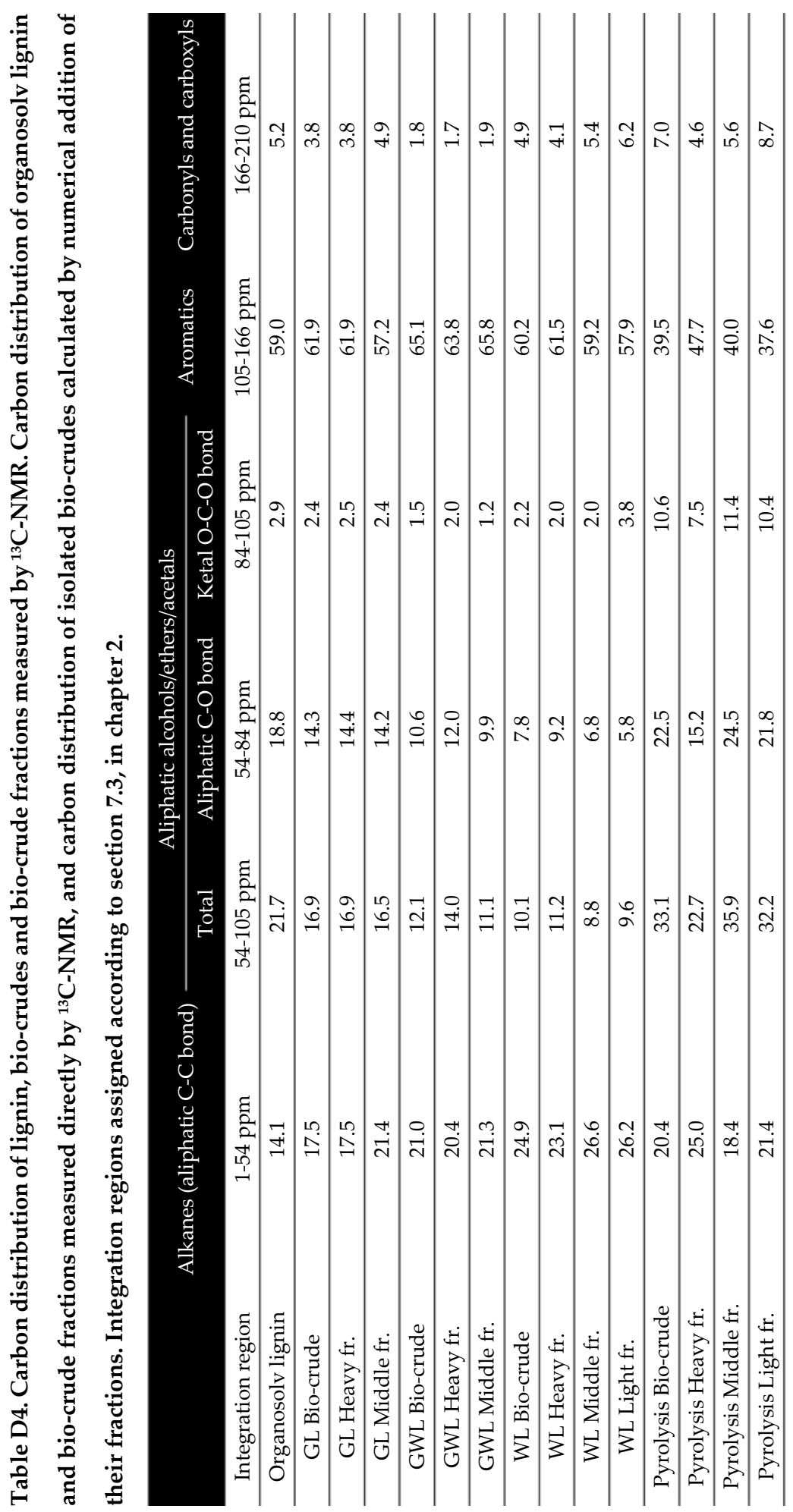




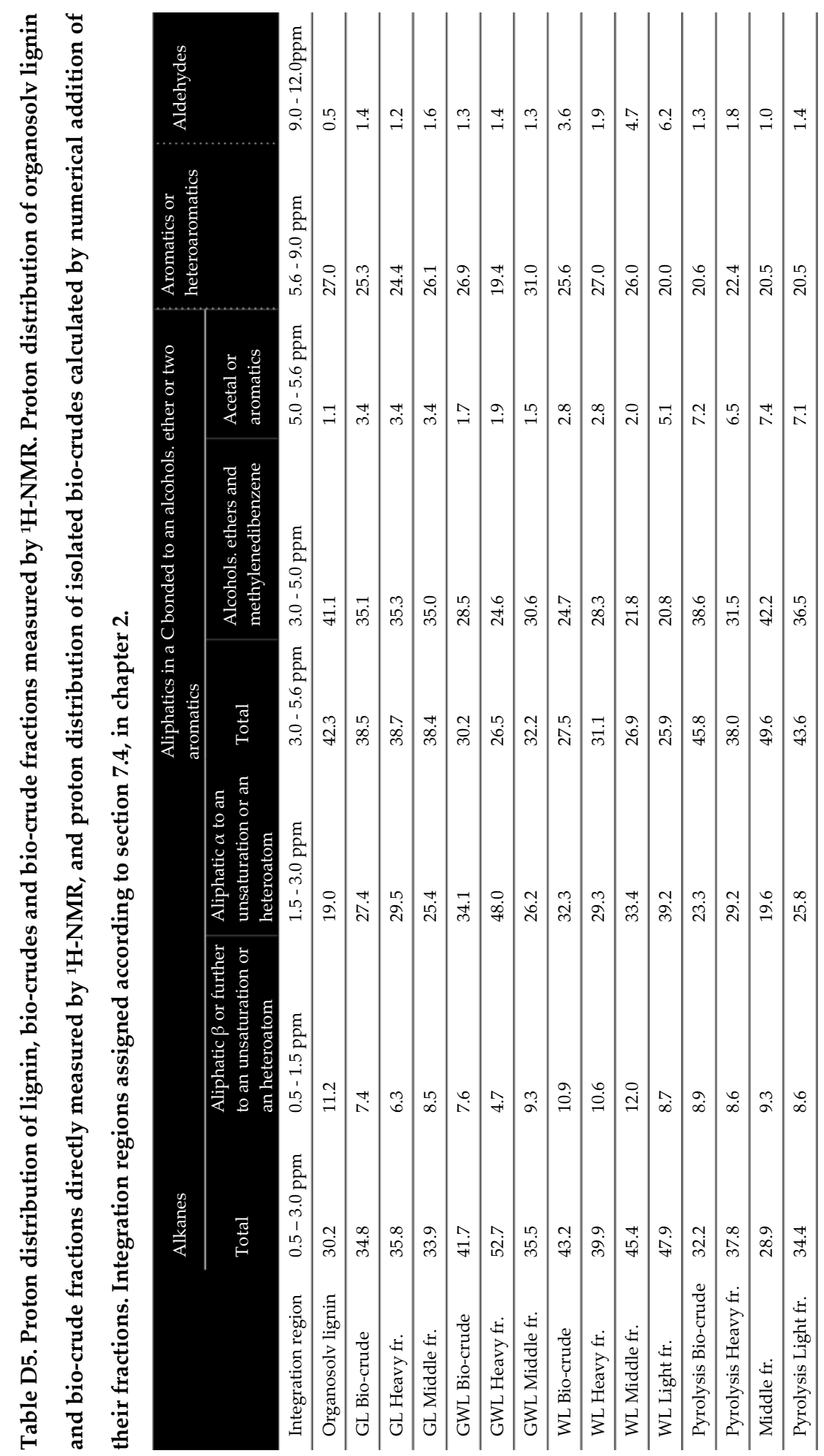




\section{D.I. Overall characterization}

Table D6. GC-MS results for WL and pyrolysis bio-crudes.

\begin{tabular}{|c|c|c|c|}
\hline $\begin{array}{l}\text { Number of the peak } \\
\text { in Figure D1 }\end{array}$ & $\begin{array}{l}\text { Retention time } \\
(\mathrm{min})\end{array}$ & Match & Compound \\
\hline 1 & 5.98 & 86 & 3-pentanone ${ }^{b}$ \\
\hline 2 & 6.65 & 86 & Hydroxyacetaldehyde $^{b}$ \\
\hline 3 & 7.40 & 91 & Crotonaldehyde ${ }^{b}$ \\
\hline 4 & 7.68 & 91 & acetic acid a;b \\
\hline 5 & 9.11 & 86 & Acetol b \\
\hline 6 & 11.75 & 93 & propanoic acid ${ }^{b}$ \\
\hline 7 & 13.71 & 80 & 3-hydropropanal b \\
\hline 8 & 14.05 & 95 & m-xylene ${ }^{a}$ \\
\hline 9 & 15.43 & 91 & p-xylene a \\
\hline 10 & 16.46 & 94 & 2-cyclopenten-1-one a;b \\
\hline 11 & 16.62 & 94 & 2-furaldehyde a; b \\
\hline 12 & 19.21 & 86 & 1-(acetyloxy)-2-Propanone ${ }^{b}$ \\
\hline 13 & 19.34 & 91 & 2-methyl-2-Cyclopenten-1-one a; b \\
\hline 14 & 20.6 & 80 & 4-Ethyl toluol a \\
\hline 15 & 22.18 & 91 & 2-Hydroxy-2-Cyclopenten-1-one ${ }^{b}$ \\
\hline 16 & 23.61 & 94 & 5-methylfurfural a; b \\
\hline 17 & 24.29 & 90 & 3-methyl-2-Cyclopenten-1-one $\mathrm{a} ; \mathrm{b}$ \\
\hline 18 & 24.59 & 90 & 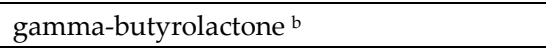 \\
\hline 19 & 25.03 & 94 & $2(5 \mathrm{H})$-furanone ${ }^{b}$ \\
\hline 20 & 25.78 & 90 & 5-Me-(5H)-Furan-2-one ${ }^{\mathrm{b}}$ \\
\hline 21 & 26.13 & 90 & 3-Hydroxy-5.6-Dihydro-(4H)-Pyran-4-one b \\
\hline 22 & 27.03 & 93 & 2-hydroxy-3-methyl-2-Cyclopenten-1-one ${ }^{b}$ \\
\hline 23 & 27.24 & 91 & 3-methyl-2(5H)-Furanone ${ }^{b}$ \\
\hline 24 & 28.98 & 94 & Phenol a; b \\
\hline 25 & 29.56 & 94 & Guaiacol a;b \\
\hline 26 & 31.33 & $90 / 97$ & 1.2-dimethoxybenzene a/o-cresol $\mathrm{b}$ \\
\hline 27 & 32.55 & 94 & Naphthalene a \\
\hline 28 & 32.67 & 91 & 4-Methyl-5H-furan-2-one ${ }^{b}$ \\
\hline 29 & 33.04 & 90 & p-cresol b \\
\hline 30 & 33.11 & 91 & m-cresol b \\
\hline 31 & 34.42 & 94 & 4-methylguaiacol a; b \\
\hline 32 & 35.26 & 93 & 2.5-dimethyl-Phenol b \\
\hline 33 & 37.64 & 94 & 1/2-methylnaphthalene a \\
\hline 34 & 38.18 & 90 & 4-ethylguaiacol a; b \\
\hline 35 & 38.36 & 95 & 1/2-methylnaphthalene a \\
\hline 36 & 41.74 & 94 & Eugenol b \\
\hline 37 & 42.38 & 95 & Dimethylnaphthalene ${ }^{a}$ \\
\hline 38 & 42.91 & 97 & Dimethylnaphthalene ${ }^{a}$ \\
\hline
\end{tabular}




\begin{tabular}{llll}
\hline $\begin{array}{l}\text { Number of the peak } \\
\text { in Figure D1 }\end{array}$ & $\begin{array}{l}\text { Retention time } \\
(\mathrm{min})\end{array}$ & Match & Compound \\
\hline 39 & 43.1 & 95 & Dimethylnaphthalene $^{\mathrm{a}}$ \\
\hline 40 & 44.1 & 93 & cis/trans-isoeugenol $^{\mathrm{b}}$ \\
\hline 41 & 46.34 & 94 & cis/trans-isoeugenol $^{\mathrm{a} ; \mathrm{b}}$ \\
\hline 42 & 47.09 & 95 & Vanillin/isovanillin $^{\mathrm{a} ; \mathrm{b}}$ \\
\hline 43 & 50.27 & 91 & Acetoguaiacon $^{\mathrm{a}} \mathrm{b}$ \\
\hline 44 & 61.5 & 96 & Coniferyl aldehyde $^{\mathrm{b}}$ \\
\hline
\end{tabular}

a Compounds present in WL bio-crude

${ }^{\mathrm{b}}$ Compounds present in pyrolysis bio-crude. 

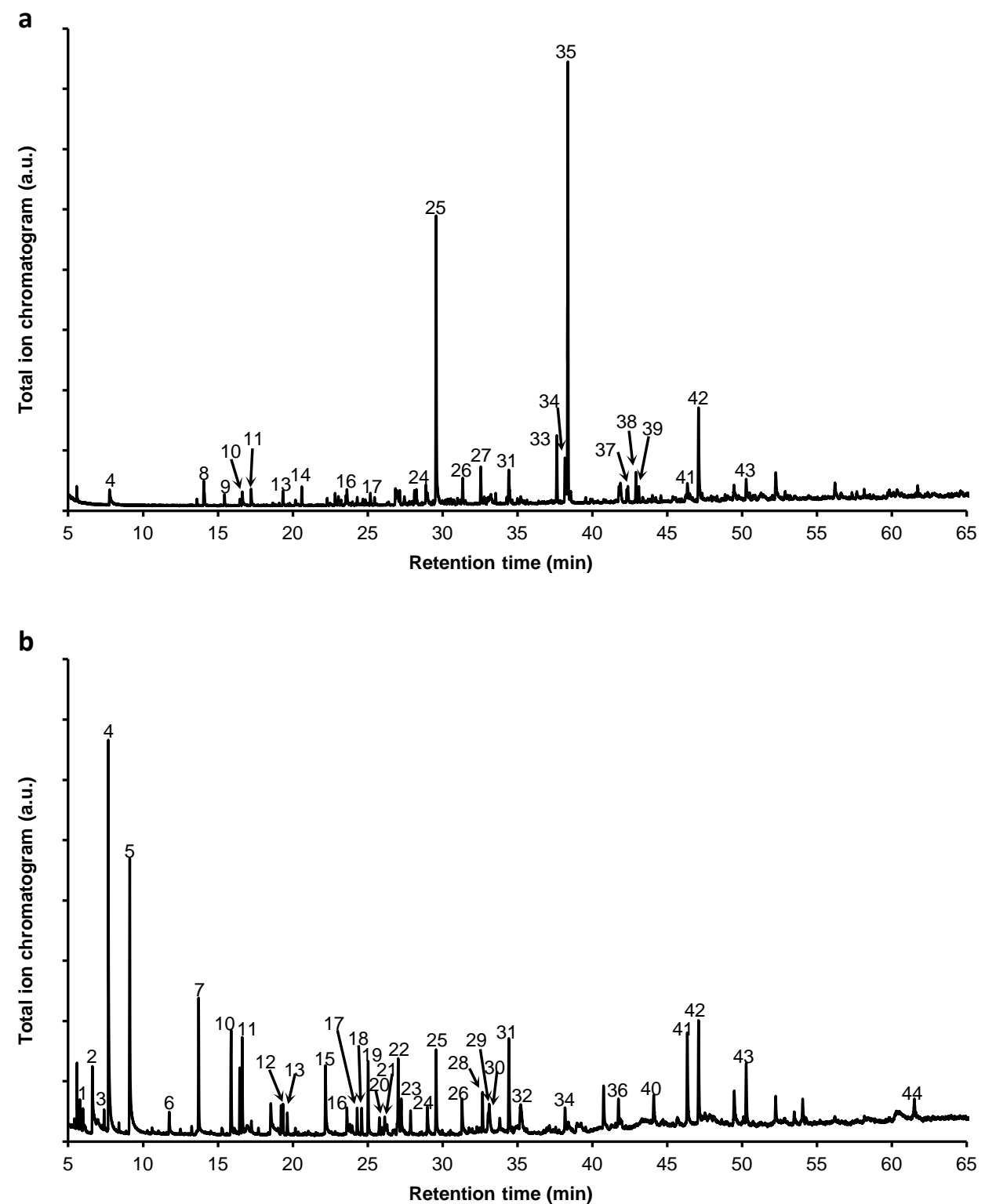

Figure D1. GC-MS spectra of WL (a) and pyrolysis (b) bio-crudes. WL performed at $300^{\circ} \mathrm{C}$ during 30 minutes with $10 w t \%$ of wood and $90 w^{\circ} \%$ of water. Pyrolysis experiment performed at $300^{\circ} \mathrm{C}$ with residence times of 20-25 minutes for the solid particles and below 2 seconds for the bio-crude. 


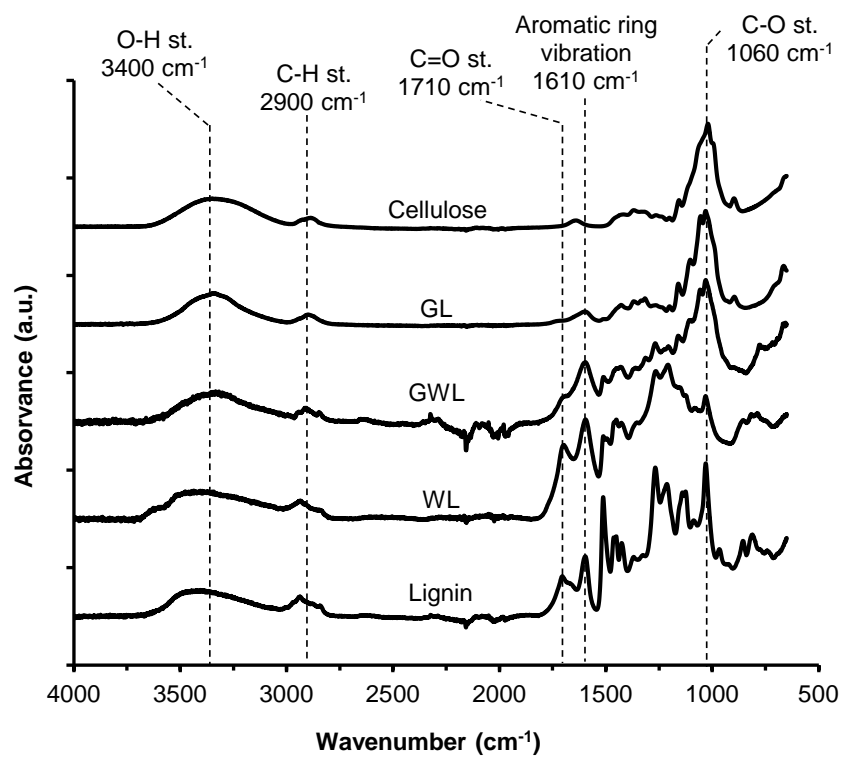

Figure D2. Normalised FTIR spectra of model components and solid residues obtained in liquefaction experiments. Liquefaction experiments performed at $300^{\circ} \mathrm{C}$ during 30 minutes with $10 \mathrm{wt} \%$ of wood and $90 \mathrm{wt} \%$ of solvent (guaiacol, guaiacol:water and water). Pyrolysis experiment performed at $300^{\circ} \mathrm{C}$ with residence times of 2025 minutes for the solid particles and below 2 seconds for the biocrude. 


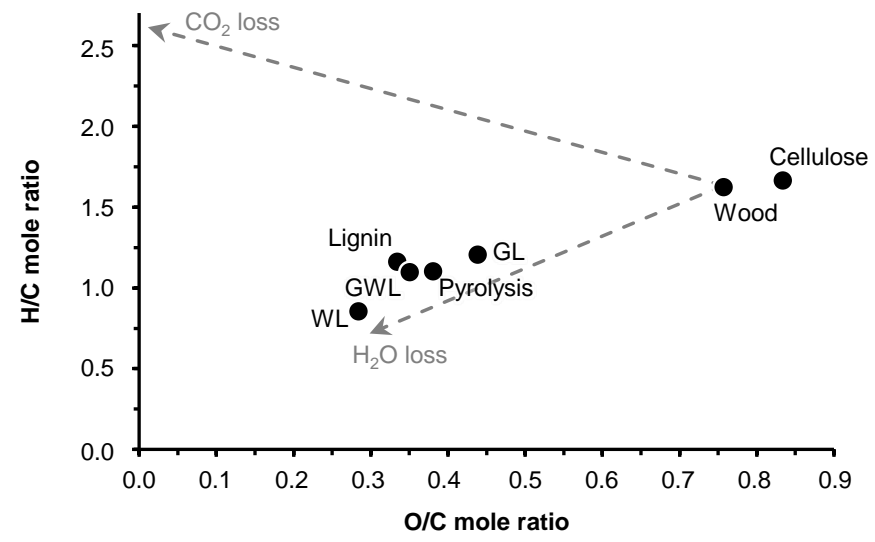

Figure D3. Van Krevelen diagram of the model components and the combined bio-crude and solid of the four experiments. Trend lines for wood dehydration and decarboxylation are also represented. Liquefaction experiments performed at $300^{\circ} \mathrm{C}$ during 30 minutes with $10 \mathrm{wt} \%$ of wood and $90 \mathrm{wt} \%$ of solvent (guaiacol, guaiacol:water and water). Pyrolysis experiment performed at $300^{\circ} \mathrm{C}$ with residence times of 20-25 minutes for the solid particles and below 2 seconds for the bio-crude. 


\section{D.2. Guaiacol as reaction medium (GL)}

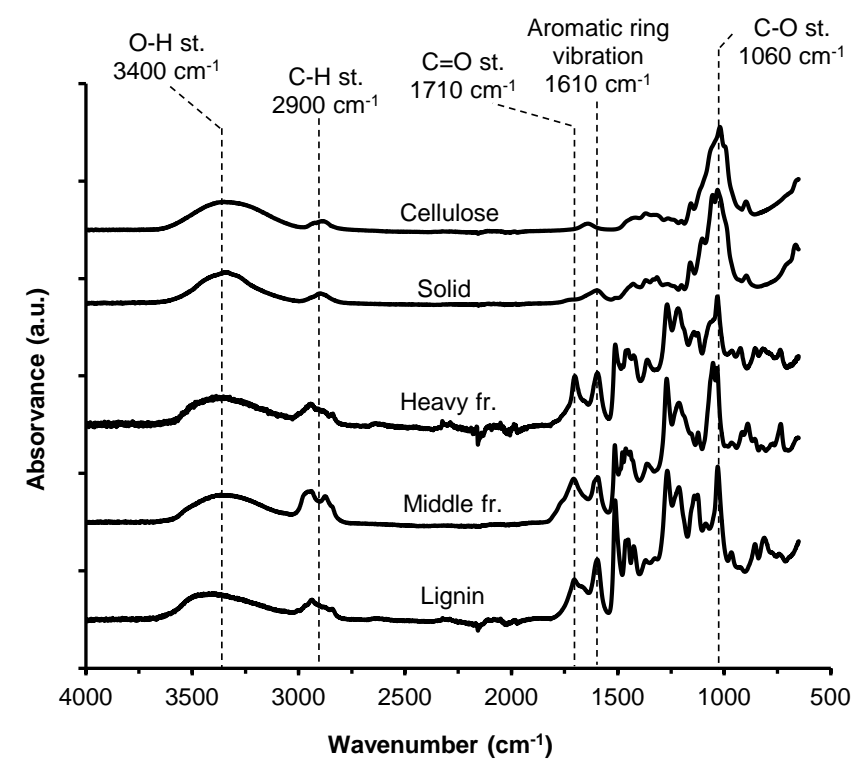

Figure D4. FTIR spectra for GL products and model components. GL performed at $300^{\circ} \mathrm{C}$ during 30 minutes with $10 \mathrm{wt} \%$ of wood and 90 $\mathbf{w t} \%$ of guaiacol. 


\section{D.3. Guaiacol:water as reaction medium (GWL)}

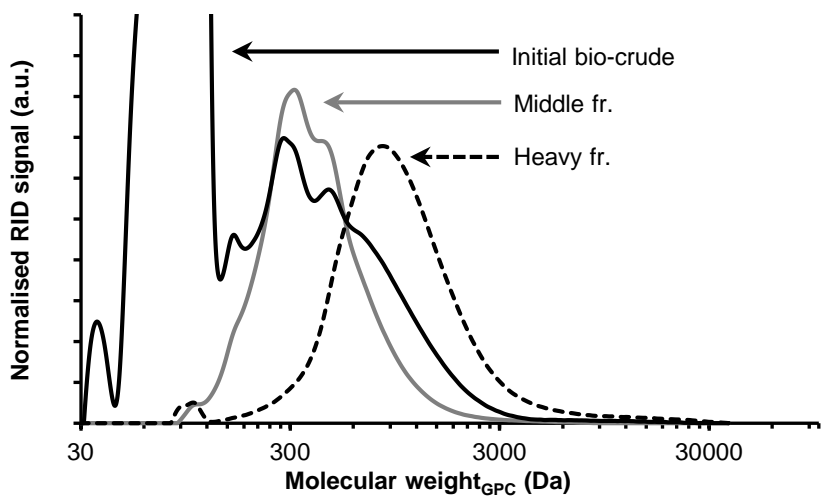

Figure D5. GPC chromatograms of GWL bio-crude and its fractions. Middle and heavy fractions have an average MwGPC around $360 \mathrm{Da}$ and 900 Da respectively (the small peak with MwgPC of 113 Da corresponds to a THF decomposition product. GWL performed at $300^{\circ} \mathrm{C}$ during 30 minutes with $10 \mathrm{wt} \%$ of wood and $45 \mathrm{wt} \%$ of guaiacol and $45 \mathrm{wt} \%$ of water).

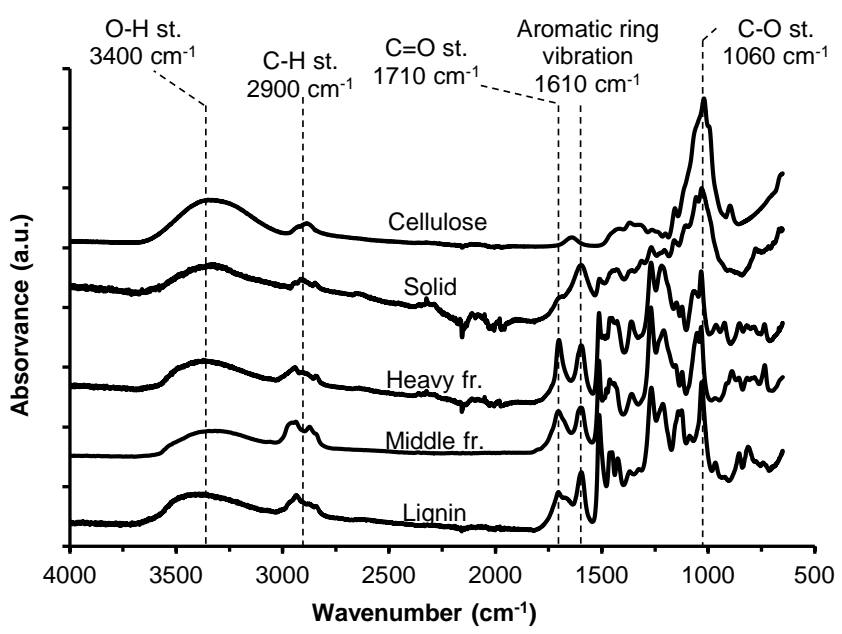

Figure D6. FTIR spectra for GWL liquefaction products and model components. GWL performed at $300^{\circ} \mathrm{C}$ during 30 minutes with $10 \mathrm{wt} \%$ of wood and $45 \mathrm{wt} \%$ of guaiacol and $45 \mathrm{wt} \%$ of water. 


\section{D.4. Water as reaction medium (WL)}

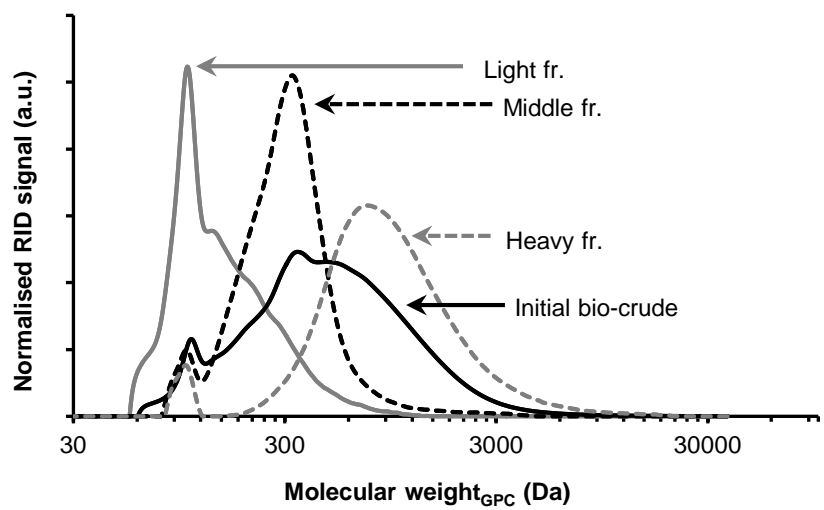

Figure D7. GPC chromatograms water liquefaction bio-crude and its fractions. Light, middle and heavy fractions have an average MwGPC around $150 \mathrm{Da}, 360 \mathrm{Da}$ and $850 \mathrm{Da}$ respectively (the small peak with MwgPc of 113 Da corresponds to a THF decomposition product. WL experiment performed at $300^{\circ} \mathrm{C}$ during 30 minutes with $10 \mathrm{wt} \%$ of wood and $90 \mathrm{wt} \%$ of water).

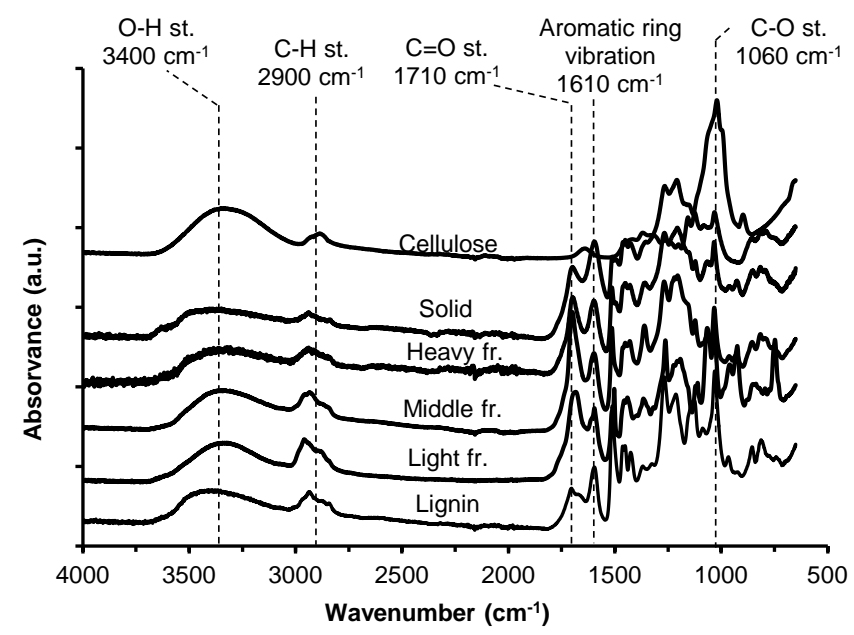

Figure D8. FTIR spectra for water liquefaction products and model components. $\mathrm{WL}$ experiment performed at $300^{\circ} \mathrm{C}$ during 30 minutes with $10 w t \%$ of wood and $90 \mathrm{wt} \%$ of water. 


\section{D.5. Pyrolysis experiment}

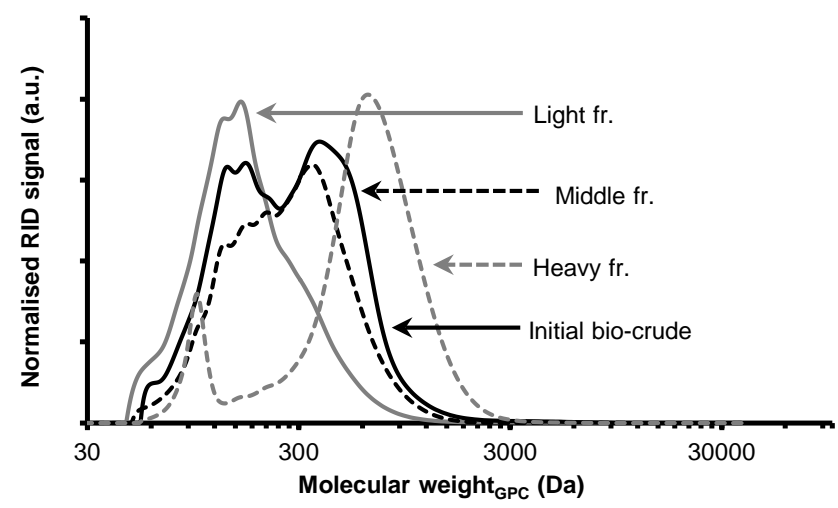

Figure D9. GPC chromatograms pyrolysis bio-crude and its fractions. Light, middle and heavy fractions have an average MwGPC around 150 Da, 250 Da and 700 Da respectively (the small peak with MwGPC of 113 Da corresponds to a THF decomposition product. Pyrolysis experiment performed at $300^{\circ} \mathrm{C}$ with residence times of 20-25 minutes for the solid particles and below 2 seconds for the bio-crude).

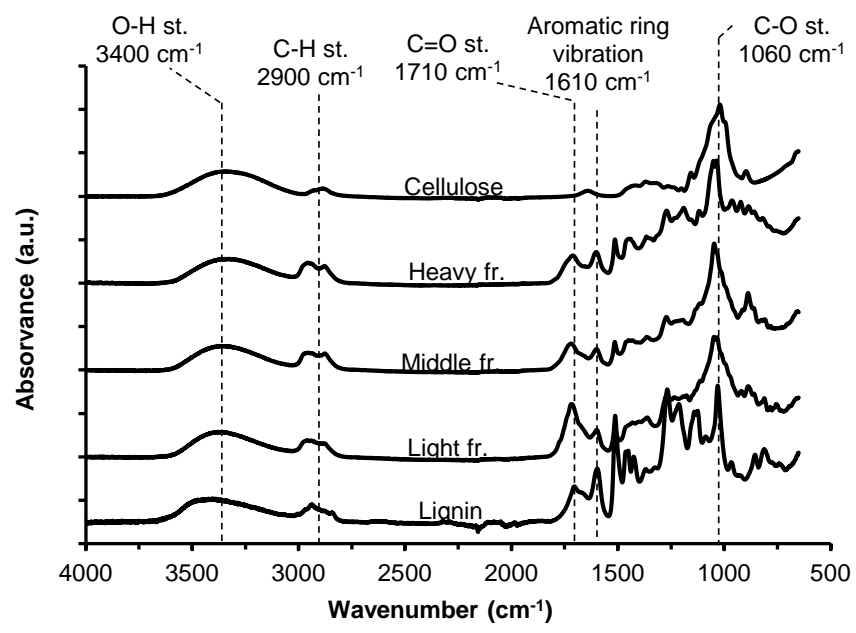

Figure D10. FTIR spectra for pyrolysis products and model components. Pyrolysis experiment performed at $300^{\circ} \mathrm{C}$ with residence times of 20-25 minutes for the solid particles and below 2 seconds for the bio-crude. 


\section{E. Optimization of wood liquefaction in methylnaphthalene}

Table E1. Product yields of the liquefaction experiments. Liquefaction experiments were performed during $10-150 \mathrm{~min}$, at $200-330^{\circ} \mathrm{C}$ and with $0-10 \mathrm{wt} \%$ of water, $10 \mathrm{wt} \%$ of biomass, and the balance with 1-methylnaphthalene $(90-80 \mathrm{wt} \%)$. Two particle sizes were tested (diameter $<150$ and $<500 \mu \mathrm{m}$ ). The experiment marked as BC corresponds to the liquefaction at the optimal conditions $\left(20 \mathrm{~min}, 300^{\circ} \mathrm{C}, 5 \mathrm{wt} \%\right.$ of water and a particle size < $500 \mu \mathrm{m})$ and it is shown four times for an easier comparison of the results.

\begin{tabular}{|c|c|c|c|c|}
\hline & & $\begin{array}{l}\text { Liquid } \\
(\mathrm{C} \%)\end{array}$ & $\begin{array}{l}\text { Gas } \\
(\mathrm{C} \%)\end{array}$ & $\begin{array}{l}\text { Solid } \\
(\mathrm{C} \%)\end{array}$ \\
\hline \multirow{4}{*}{$\begin{array}{l}\text { Time } \\
\left(5 \mathrm{wt} \% \text { water, } 300^{\circ} \mathrm{C},<500 \mu \mathrm{m}\right)\end{array}$} & $10 \mathrm{~min}$ & 82.1 & 5.2 & 12.7 \\
\hline & $20 \min (B C)$ & 86.0 & 6.9 & 7.2 \\
\hline & $30 \mathrm{~min}$ & 86.3 & 6.8 & 6.9 \\
\hline & $150 \mathrm{~min}$ & 82.7 & 11.6 & 5.7 \\
\hline \multirow{3}{*}{$\begin{array}{l}\text { Water content } \\
\left(20 \mathrm{~min}, 300^{\circ} \mathrm{C},<500 \mu \mathrm{m}\right)\end{array}$} & $0 \mathrm{wt} \%$ & 74.7 & 5.1 & 20.2 \\
\hline & $5 \mathrm{wt} \%(\mathrm{BC})$ & 86.0 & 6.9 & 7.2 \\
\hline & $10 \mathrm{wt} \%$ & 89.2 & 6.3 & 4.4 \\
\hline \multirow{4}{*}{$\begin{array}{l}\text { Temperature } \\
(20 \mathrm{~min}, 5 \mathrm{wt} \% \text { water, }<500 \mu \mathrm{m})\end{array}$} & $200^{\circ} \mathrm{C}$ & 28.0 & 0.0 & 72.0 \\
\hline & $270^{\circ} \mathrm{C}$ & 81.2 & 1.8 & 17.1 \\
\hline & $300^{\circ} \mathrm{C}(\mathrm{BC})$ & 86.0 & 6.9 & 7.2 \\
\hline & $330^{\circ} \mathrm{C}$ & 86.6 & 9.0 & 4.4 \\
\hline \multirow{2}{*}{$\begin{array}{l}\text { Particle size of wood } \\
\left(20 \mathrm{~min}, 5 \mathrm{wt} \% \text { water and } 300^{\circ} \mathrm{C}\right)\end{array}$} & $<500 \mu \mathrm{m}(\mathrm{BC})$ & 86.0 & 6.9 & 7.2 \\
\hline & $<150 \mu \mathrm{m}$ & 85.9 & 6.2 & 7.9 \\
\hline
\end{tabular}

A fast parameter screening was performed to find the optimal conditions for the liquefaction of pinewood in 1-methylnaphthalene in terms of bio-crude yield and wood conversion (results presented in Table E1). Reaction time was varied from 10 to 30 minutes, water concentration from 0 to $10 \mathrm{wt} \%$, and temperature from 200 to $330^{\circ} \mathrm{C}$. A wood concentration of $10 \mathrm{wt} \%$ and an initial $\mathrm{N}_{2}$ pressure of 6 bar were used for all the experiments. Optimal conditions were found to be 20 minutes, a $5 \mathrm{wt} \%$ of water concentration and a reaction temperature of $300^{\circ} \mathrm{C}$. Lower reaction time, water concentration or reaction temperature resulted in incomplete wood conversion (sugar fingerprint was observed in the FTIR spectra of the produced solids), while 
higher values of these parameters did not significantly improve the bio-crude yield. Furthermore, the increase of water concentration from 5 to $10 \mathrm{wt} \%$ resulted in an increase in the process pressure (from 55 to 70 bar). Results also showed that the particle size of the wood particles has no effect on the product distribution. 


\section{F. Tables and figures (Chapter 5)}

Table F1. Elemental composition and $\mathrm{H} / \mathrm{C}$ effective ratio of carbohydrates and lignin liquefaction products. Liquefaction experiment was performed at $300-310^{\circ} \mathrm{C}$ with $10 \mathrm{wt} \%$ of biomass, $5 \mathrm{wt} \%$ of water and $85 \mathrm{wt} \%$ of 1-methylnaphthalene during $20 \mathrm{~min}$.

\section{$\mathrm{N}(w \mathrm{t} \%)\left({ }^{*}\right) \quad \mathrm{C}(\mathrm{wt} \%) \quad \mathrm{H}(\mathrm{wt} \%) \quad \mathrm{O}(\mathrm{wt} \%) \quad \mathrm{H} / \mathrm{C}$ eff}

Cellulose

\begin{tabular}{llllll} 
Unreacted & 0.0 & 42.9 & 6.2 & 50.8 & -0.04 \\
\hline Tar & 0.6 & 57.6 & 5.9 & 35.9 & 0.29 \\
\hline Oil & 1.5 & 70.9 & 6.5 & 21.1 & 0.65 \\
\hline Bio-crude & 1.4 & 70.9 & 6.4 & 21.3 & 0.62 \\
\hline Solid & 0.9 & 70.6 & 4.3 & 24.2 & 0.21 \\
\hline Amylopectin & & & & & \\
Unreacted & 0.0 & 40.5 & 6.4 & 53.1 & -0.08 \\
\hline Tar & 0.5 & 58.6 & 6.1 & 34.9 & 0.34 \\
\hline Oil & 1.1 & 65.5 & 6.8 & 26.7 & 0.63 \\
\hline Bio-crude & 1.0 & 65.7 & 6.7 & 26.5 & 0.61 \\
\hline Solid & 0.5 & 71.4 & 4.6 & 23.5 & 0.28 \\
\hline Lignin & & & & & \\
Unreacted & 0.1 & 65.8 & 6.3 & 27.8 & 0.50 \\
\hline Tar & 0.2 & 66.6 & 6.4 & 26.8 & 0.53 \\
\hline Oil & 0.2 & 66.8 & 7.5 & 25.5 & 0.77 \\
\hline Bio-crude & 0.3 & 66.9 & 7.2 & 25.7 & 0.70 \\
\hline & & & & & \\
\hline
\end{tabular}

$\left.{ }^{*}\right)$ the $\mathrm{N}$ content is higher in the products than in the feedstock because the product is contaminated by $\mathrm{N}$-components present in the 1-methylnaphthalene. 


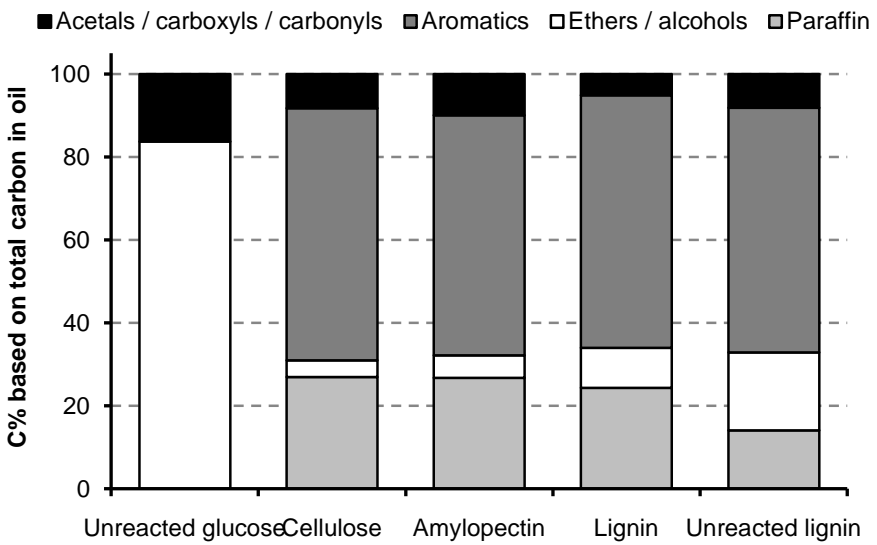

Figure F1. ${ }^{13} \mathrm{C}-\mathrm{NMR}$ integration results of unreacted lignin and solvent-free liquefaction bio-oils. Integration regions for ${ }^{13} \mathrm{C}-\mathrm{NMR}$ assigned according to section 7.3 in chapter 2 , with the exception of the acetals (84 - $105 \mathrm{ppm})$, which are combined with the carbonyl/carboxyl carbon. Liquefaction experiments were performed at $300-310^{\circ} \mathrm{C}$ with a $10 \mathrm{wt} \%$ of biomass, a $5 \mathrm{wt} \%$ of water, an $85 \mathrm{wt} \%$ of 1-methylnaphthalene during $20 \mathrm{~min}$. 


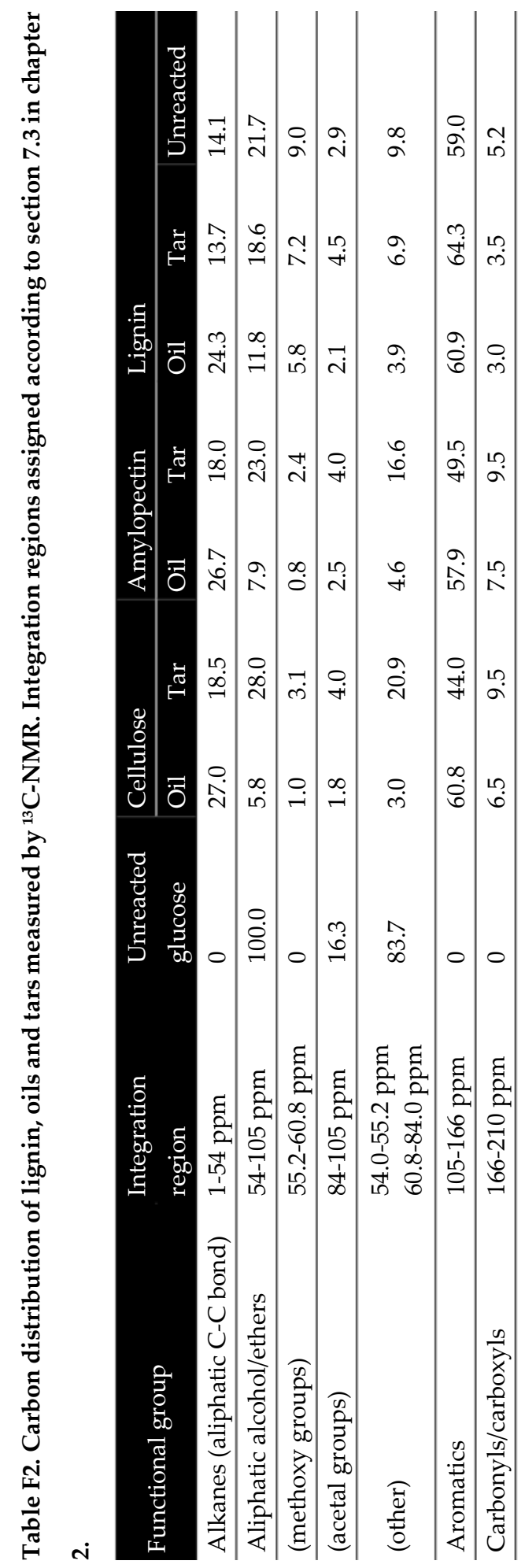




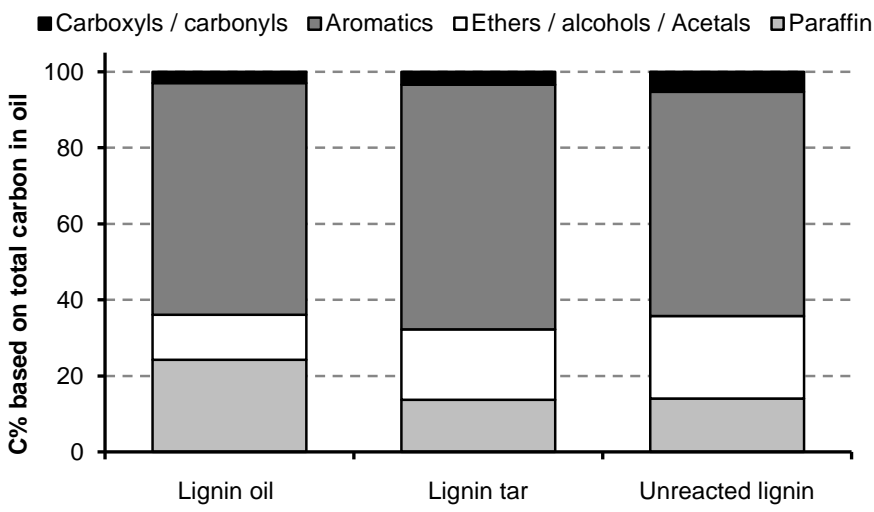

Figure F2. ${ }^{13} \mathrm{C}-\mathrm{NMR}$ integration results of unreacted lignin and lignin derived (solvent-free) bio-oil and bio-tar. Integration regions for ${ }^{13} \mathrm{C}$ NMR assigned according to section 7.3 in chapter 2. Liquefaction experiment was performed at $300-310^{\circ} \mathrm{C}$ with $10 \mathrm{wt} \%$ of organosolv lignin, $5 \mathrm{wt} \%$ of water and $85 \mathrm{wt} \%$ of 1-methylnaphthalene during 20 $\min$.

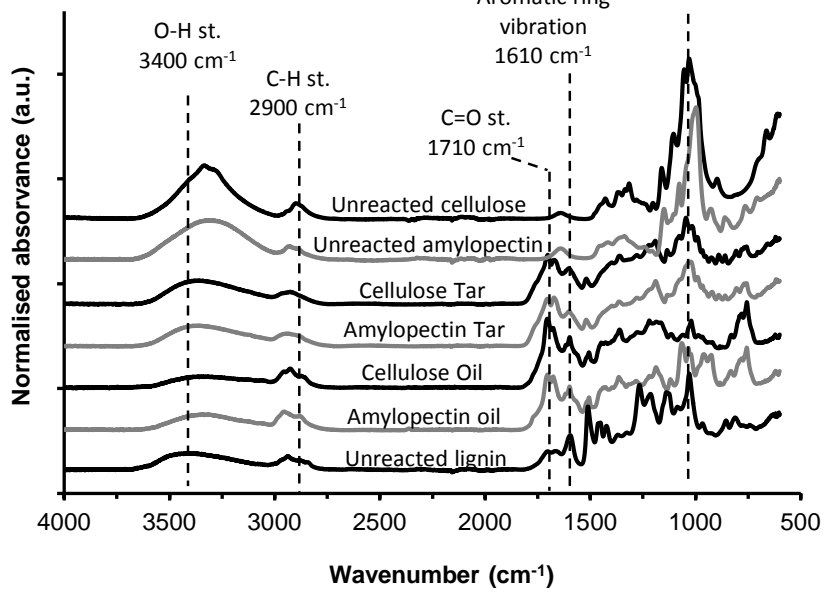

Figure F3. FTIR spectra of isolated bio-oils and bio-tars from cellulose and amylopectin. Liquefaction experiments were performed at 300$310^{\circ} \mathrm{C}$ with $10 \mathrm{wt} \%$ of cellulose or amylopectin, $5 \mathrm{wt} \%$ of water and 85 wt $\%$ of 1-methylnaphthalene during $20 \mathrm{~min}$. 


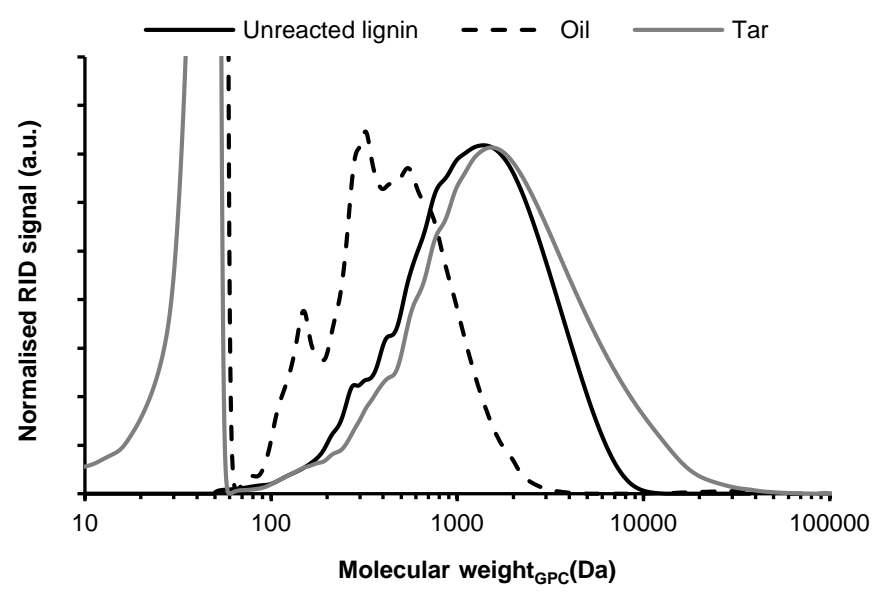

Figure F4. Molecular weight distribution of unreacted lignin, lignin oil and lignin tar. Lignin liquefaction performed at $300-310^{\circ} \mathrm{C}$ with 10 $w t \%$ of organosolv lignin, $5 \mathrm{wt} \%$ of water and $85 \mathrm{wt} \%$ of 1methylnaphthalene during $20 \mathrm{~min}$.

a

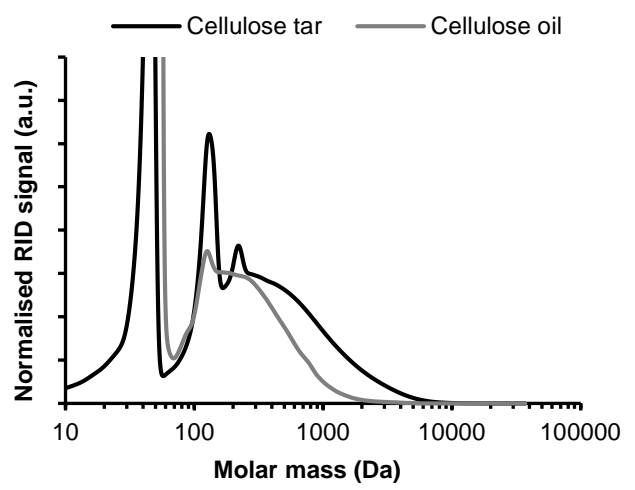

b

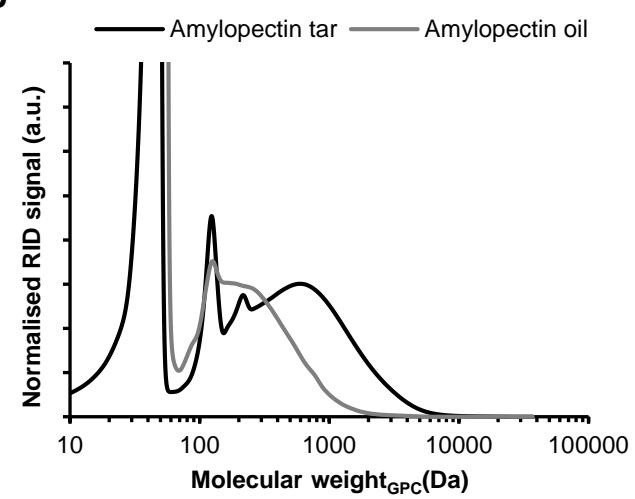

Figure F5. Molecular weight distribution of (a) cellulose and (b) amylopectin oils and tars. Liquefaction performed at $300-310^{\circ} \mathrm{C}$ with $10 \mathrm{wt} \%$ of biomass, $5 \mathrm{wt} \%$ of water and $85 \mathrm{wt} \%$ of 1-methylnaphthalene during $20 \mathrm{~min}$. 


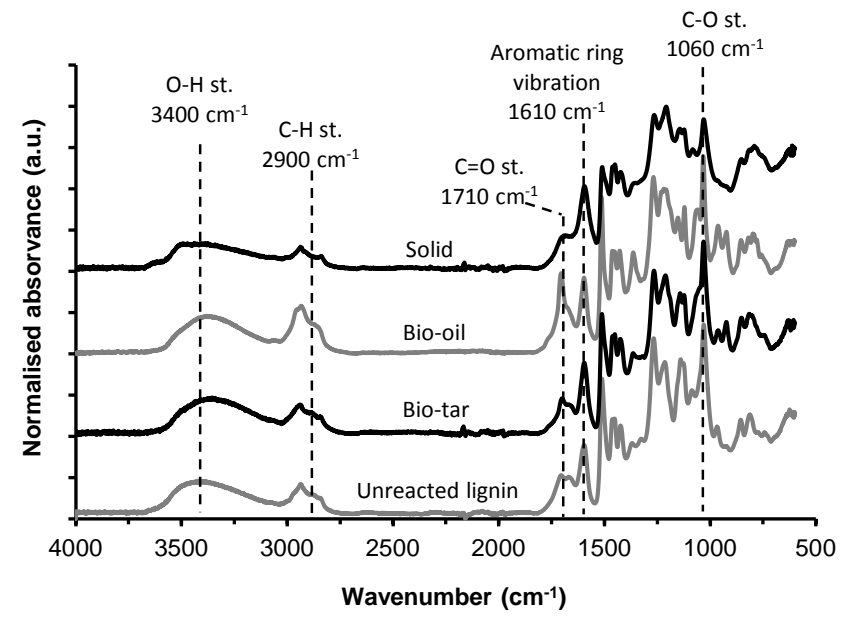

Figure F6. FTIR spectra of unreacted lignin and liquefaction products. Liquefaction experiment was performed at $300-310^{\circ} \mathrm{C}$ with $10 \mathrm{wt} \%$ of organosolv lignin, $5 \mathrm{wt} \%$ of water and $85 \mathrm{wt} \%$ of 1 methylnaphthalene during $20 \mathrm{~min}$. 

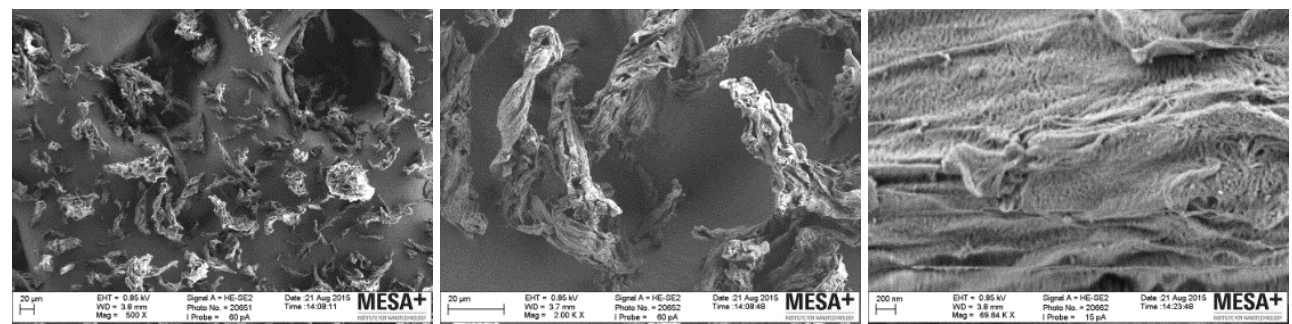

Figure F7. SEM of cellulose char. Liquefaction performed with $10 \mathrm{wt} \%$ of cellulose, $5 \mathrm{wt} \%$ of water and $85 \mathrm{wt} \%$ of 1-methylnaphthalene at $300-310^{\circ} \mathrm{C}$ for $20 \mathrm{~min}$. The background with holes observed on the left image corresponds to the tape used to fix the sample during SEM analysis.
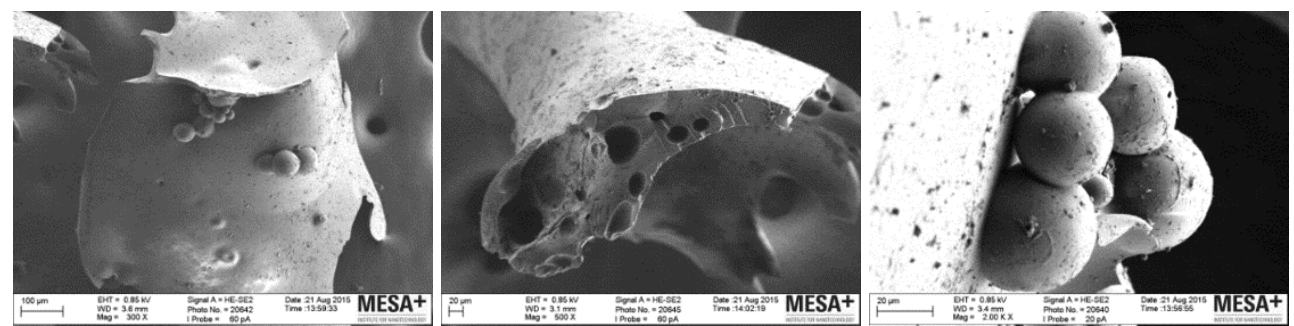

Figure F8. SEM of amylopectin char. Liquefaction performed with $10 \mathrm{wt} \%$ of amylopectin, $5 \mathrm{wt} \%$ of water and $85 \mathrm{wt} \%$ of 1-methylnaphthalene at $300-310^{\circ} \mathrm{C}$ for $20 \mathrm{~min}$. The shattering of the particles was caused by the grinding performed during sample preparation for FTIR analysis.
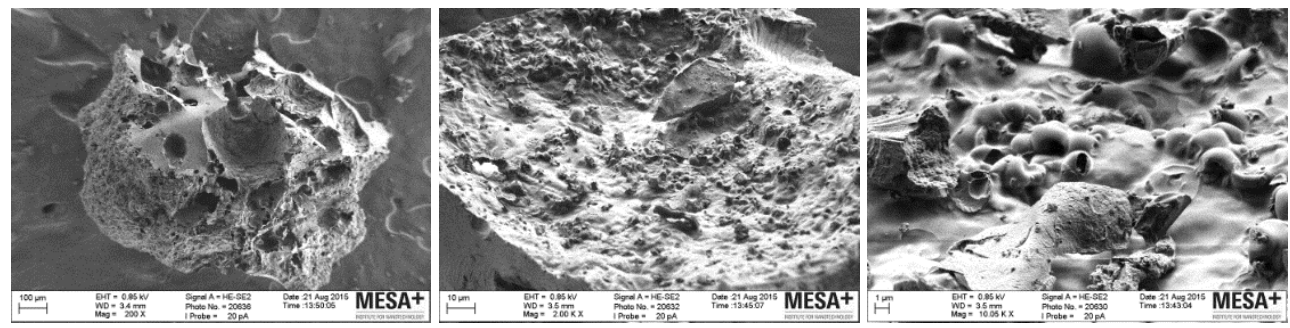

Figure F9. SEM of glucose char. Liquefaction performed with $10 \mathrm{wt} \%$ of glucose, $5 \mathrm{wt} \%$ of water and $85 \mathrm{wt} \%$ of 1-methylnaphthalene at $300-310^{\circ} \mathrm{C}$ for $20 \mathrm{~min}$. The shattering of the particles was caused by the grinding performed during sample preparation for FTIR analysis. 


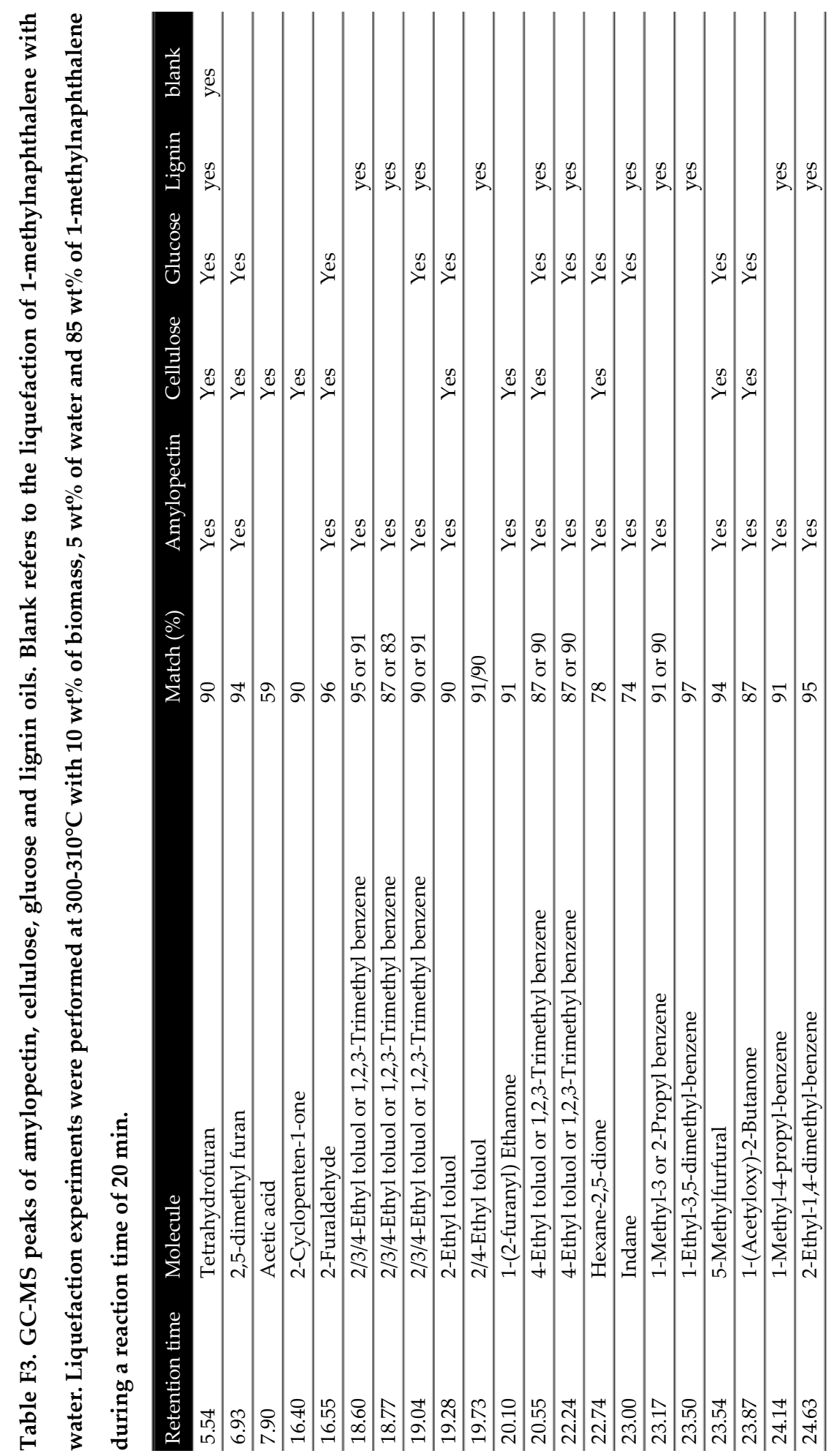




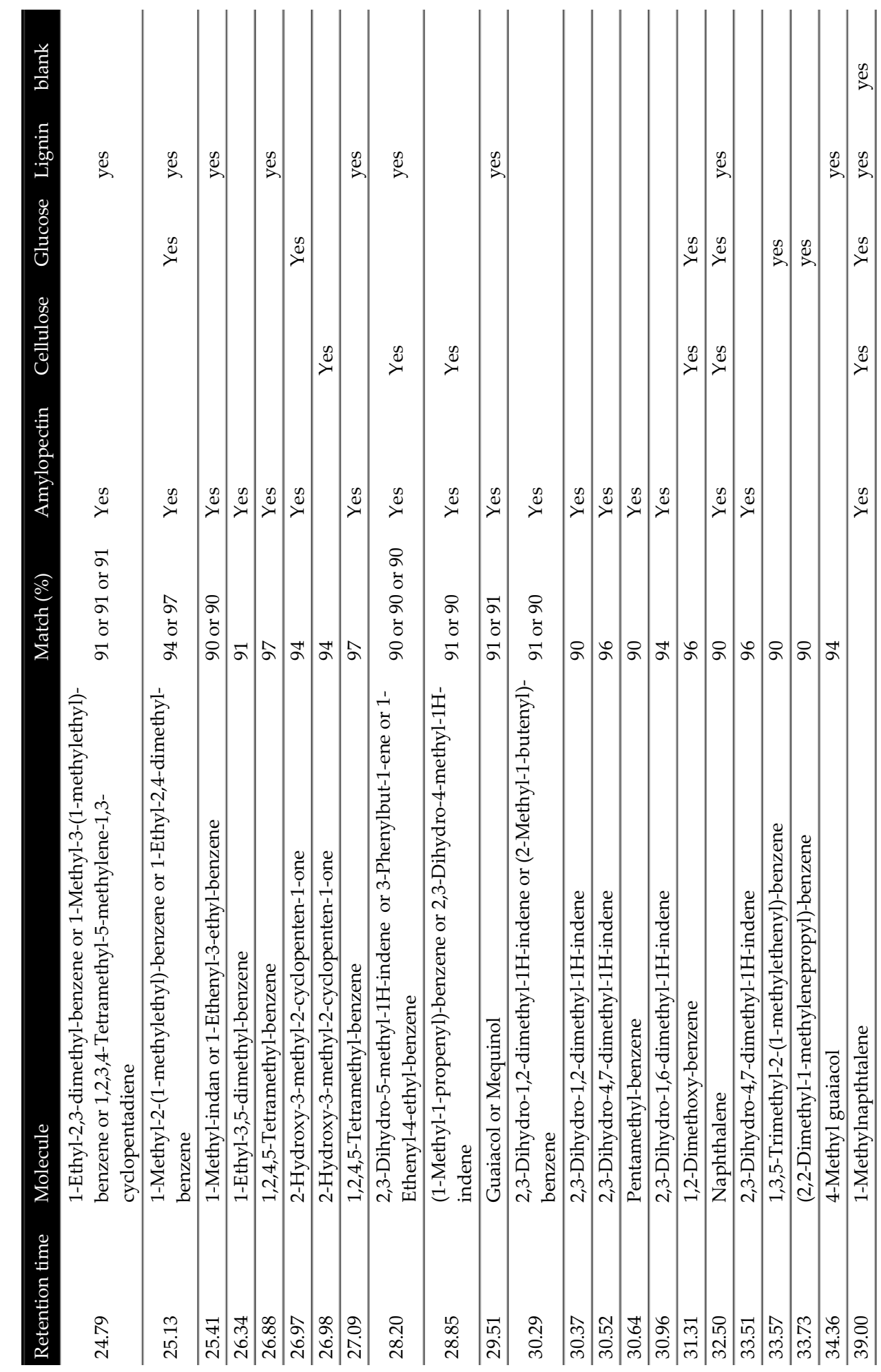

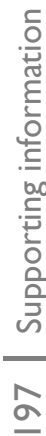




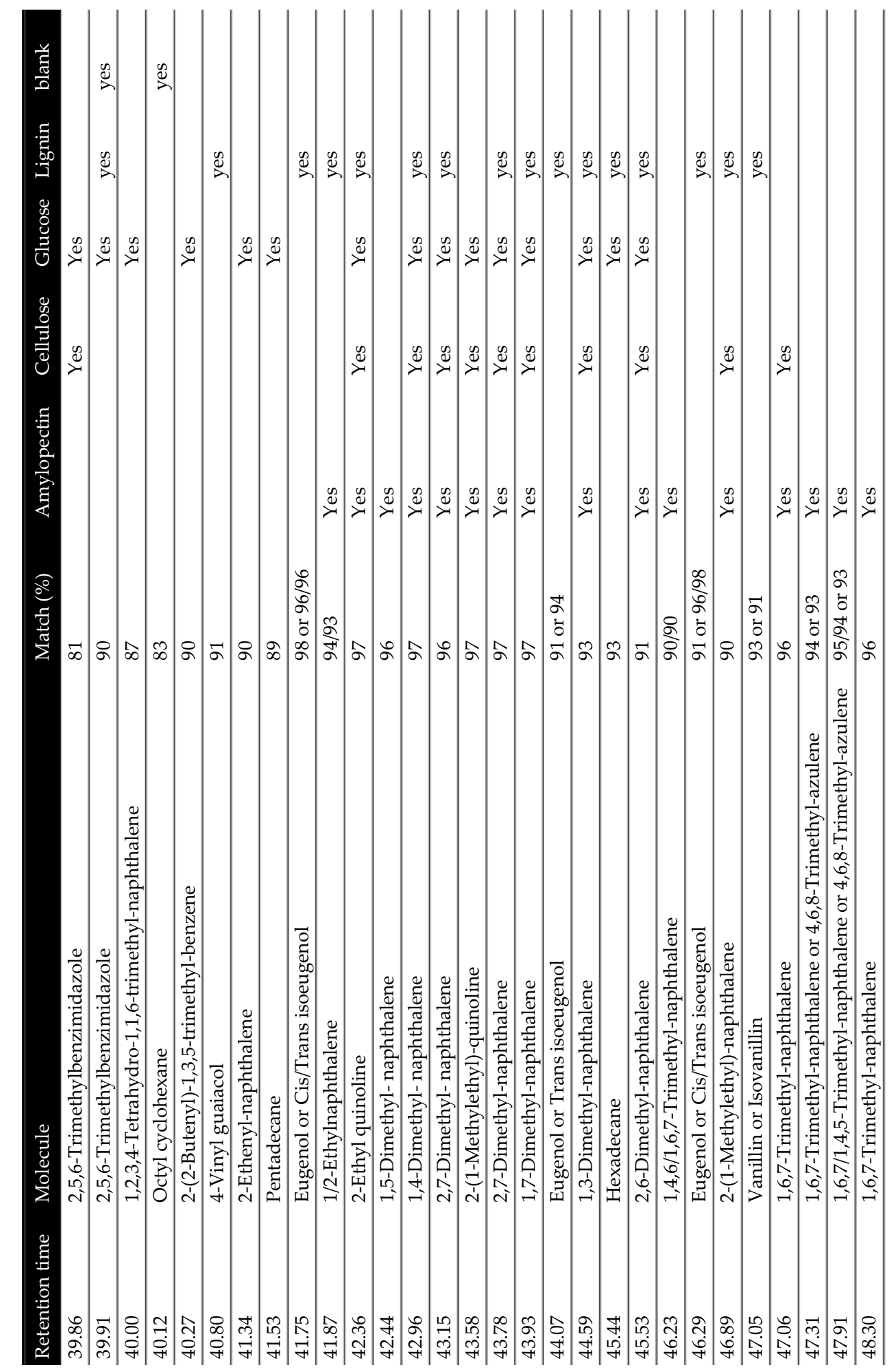




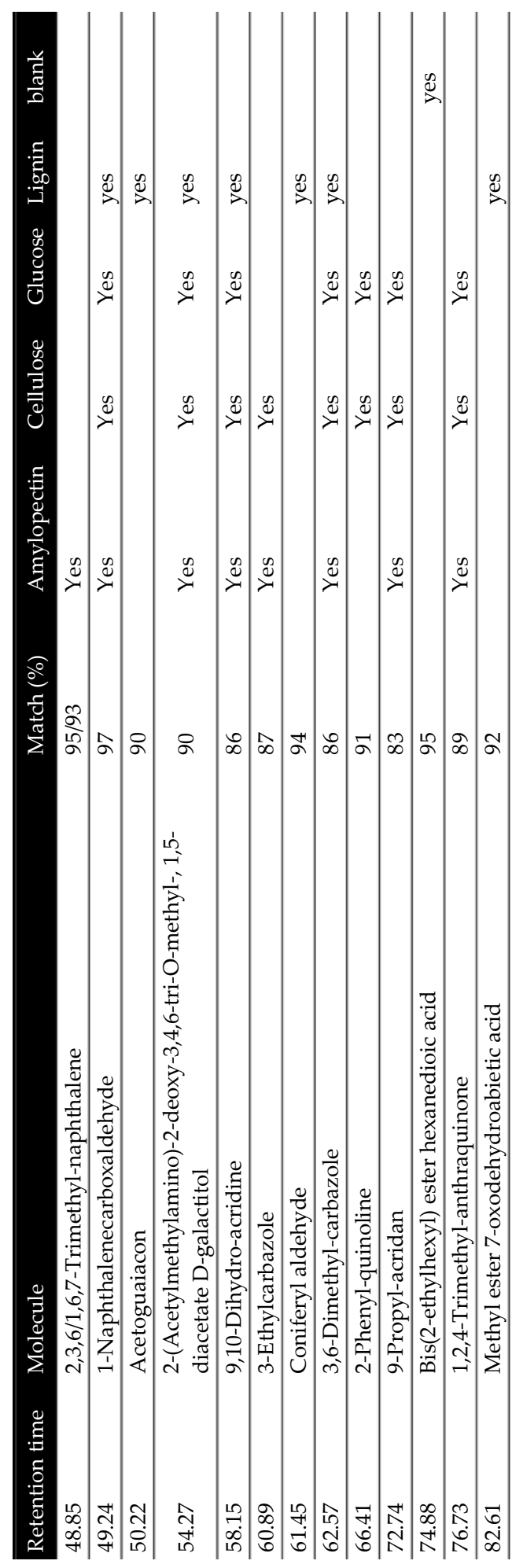



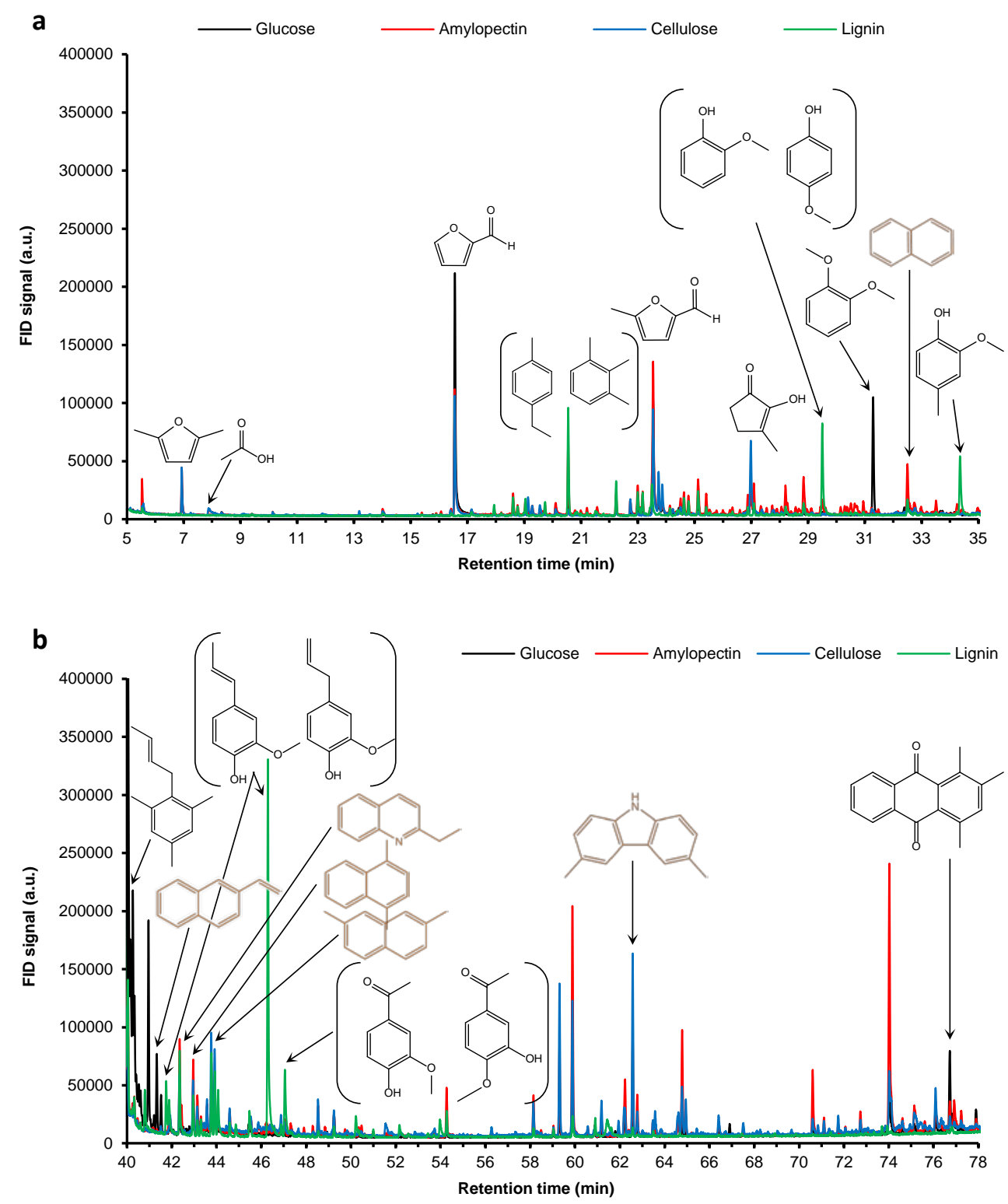

Figure F10. GC-MS chromatograms of carbohydrates and lignin oils. Major known compounds are indicated. Compounds in orange were attributed to the liquefaction solvent. Only the peak corresponding to 1-methylnapthalene appeared between the minutes 35 and 40. 


\section{G. Solid structure}

FTIR analysis showed that most of the solvents led to solid residues with a char-like structure (Figure H3, Supporting information). This type of solid has a lignin-type aromatic structure with characteristic peaks in the range 1400-1600 $\mathrm{cm}^{-1}$ (aromatic ring vibration), at $1700 \mathrm{~cm}^{-1}$ (carbonyl or carboxyl peak), a broad collection of bands at $1100-1300 \mathrm{~cm}^{-1}$ (likely aromatic C-O and C-C stretching) and over $2900 \mathrm{~cm}^{-1}$ (Caromatic-H stretching). In contrast, most of the experiments performed in the presence of alcohol groups in the liquefaction medium yielded a cellulose-like solid that presented typical carbohydrate bands just over $1000 \mathrm{~cm}^{-1}$ (aliphatic C-O stretching), under $2900 \mathrm{~cm}^{-1}$ (Caliphatic-H stretching) and at $3400 \mathrm{~cm}^{-1}(\mathrm{O}-\mathrm{H}$ stretching).

SEM analysis of few solid residues partly confirms the FTIR results (Figure G1). The carbohydrate-like solid obtained in guaiacol showed the same fibrous structure as present in wood (Figure G1a and c). A closer look at these fibres revealed the presence of cellulose microfibrils, which did not decompose during liquefaction (Figure G1d). However, the solids from water, 1-methylnaphthalene and decalin liquefactions, which are lignin-like according to FTIR, also presented the same fibrous structure as the wood (Figure G1e-j). This suggests that char is formed via direct dehydration of the wood fibres and not by excessive condensation of biocrude products. Indeed, solids derived from bio-crude condensation are expected to have lost the initial fibrous structure of wood and have rather adopted globular or glassy structures as reported elsewhere (amylopectin and glucose chars in chapter 5) [1-6]. The very small presence of glassy or globular structures in the char residues (Figure G2) suggests that formation of secondary char is minimal. 

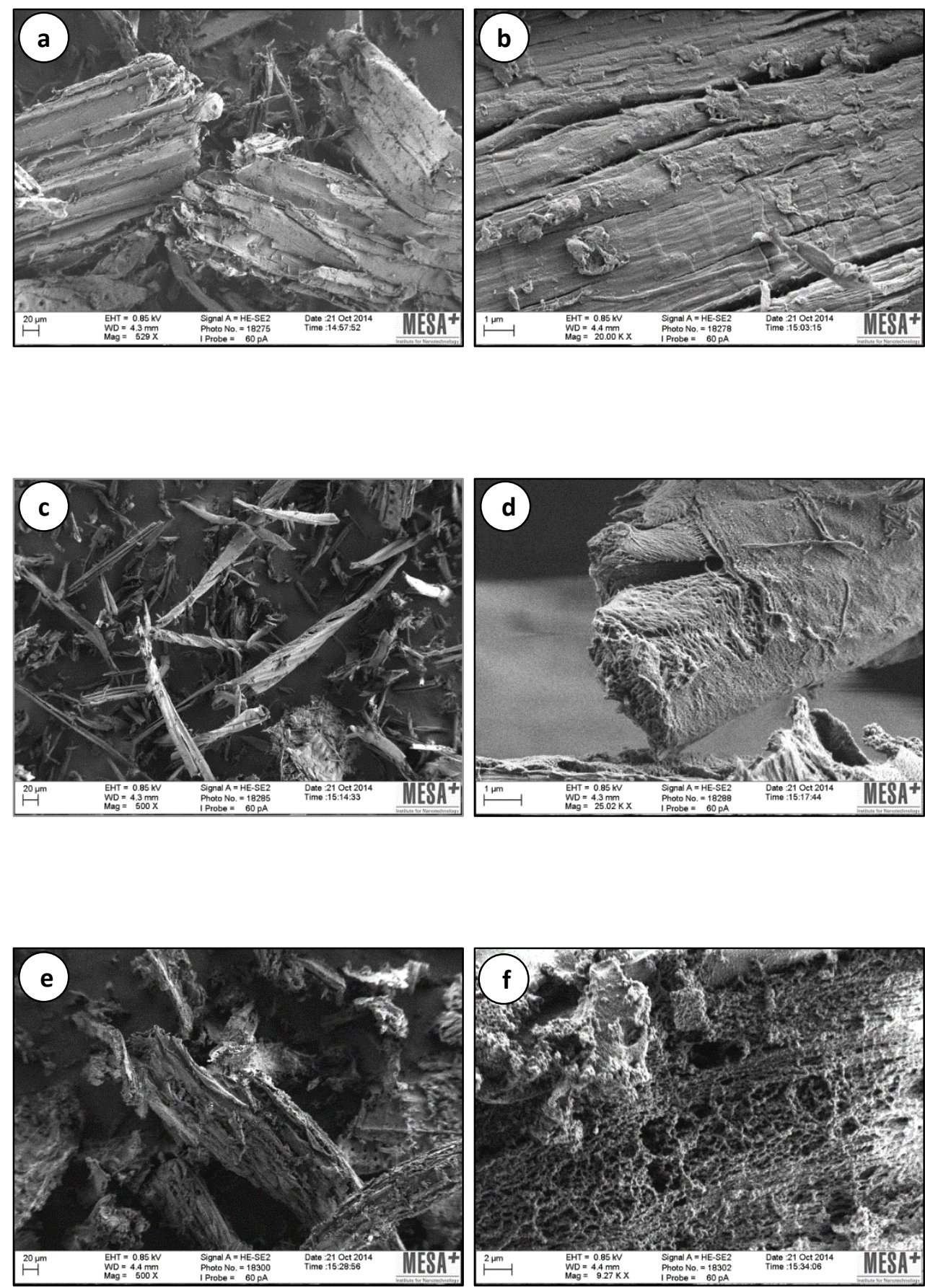

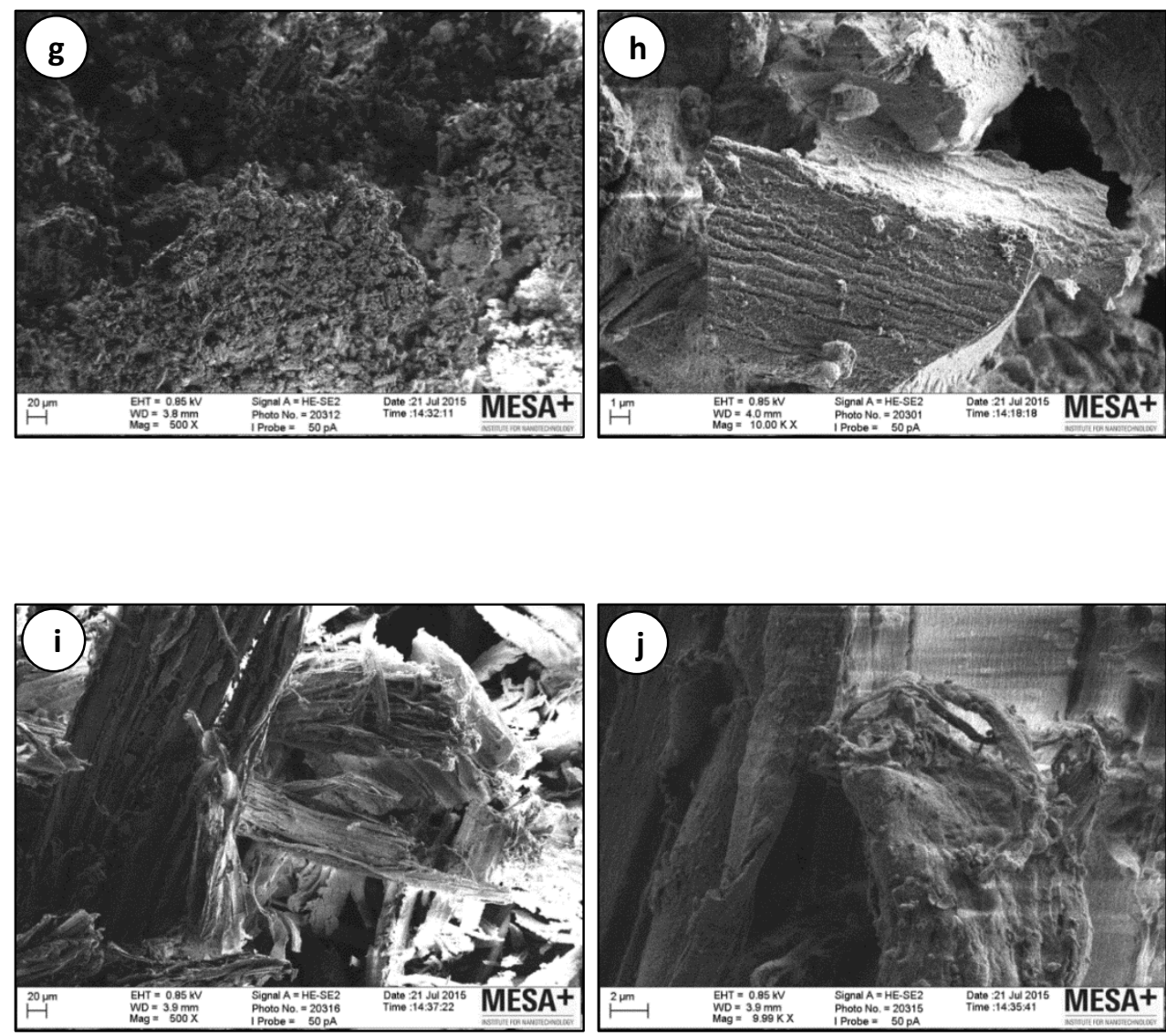

Figure G1. SEM images of wood ( $a$ and $b$ ) and solid residues obtained after liquefaction in guaiacol (c and d), water (e and f), 1-methylnaphthalene ( $g$ and $h$ ) and decalin ( $i$ and $j)$. 


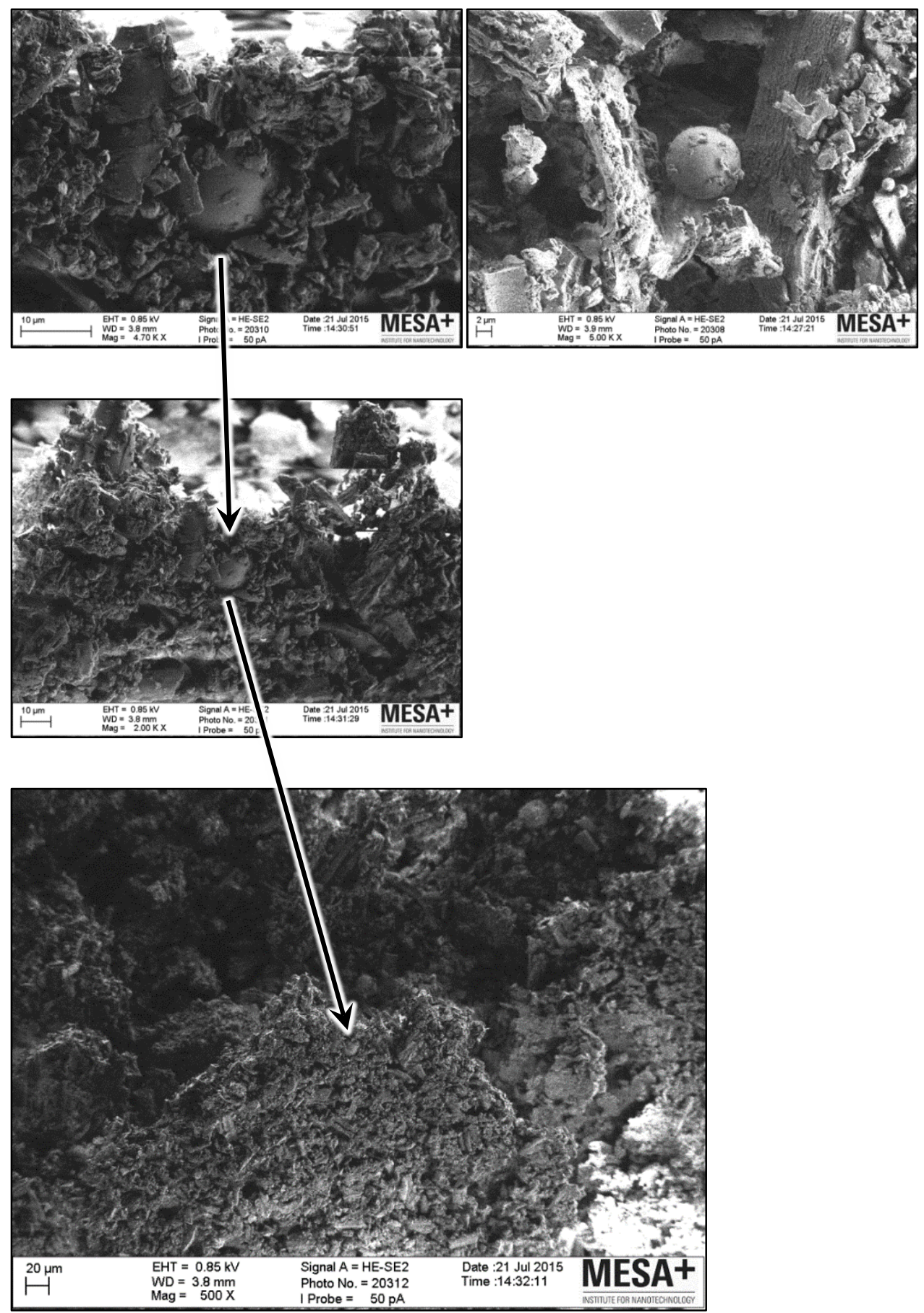

Figure G2. SEM images of the solid residue obtained after liquefaction in 1methylnaphthalene showing the minor presence of globular structures. 


\section{G.I. References}

1. Patil, S.K.R. and Lund, C.R.F., Formation and Growth of Humins via Aldol Addition and Condensation during Acid-Catalyzed Conversion of 5-Hydroxymethylfurfural. Energy \& Fuels, 2011. 25(10): p. 4745-4755.

2. Patil, S.K.R., Heltzel, J., and Lund, C.R.F., Comparison of Structural Features of Humins Formed Catalytically from Glucose, Fructose, and 5-Hydroxymethylfurfuraldehyde. Energy \& Fuels, 2012. 26(8): p. 5281-5293.

3. van Zandvoort, I., Wang, Y., Rasrendra, C.B., van Eck, E.R.H., Bruijnincx, P.C.A., Heeres, H.J., and Weckhuysen, B.M., Formation, Molecular Structure, and Morphology of Humins in Biomass Conversion: Influence of Feedstock and Processing Conditions. ChemSusChem, 2013. 6(9): p. 1745-1758.

4. Hoang, T.M.C., van Eck, E.R.H., Bula, W.P., Gardeniers, J.G.E., Lefferts, L., and Seshan, K., Humin based by-products from biomass processing as a potential carbonaceous source for synthesis gas production. Green Chemistry, 2015. 17(2): p. 959-972.

5. Sevilla, M. and Fuertes, A.B., Chemical and structural properties of carbonaceous products obtained by hydrothermal carbonization of saccharides. Chemistry - A European Journal, 2009. 15(16): p. 4195-4203.

6. Wang, Z., McDonald, A.G., Westerhof, R.J.M., Kersten, S.R.A., Cuba-Torres, C.M., Ha, S., Pecha, B., and Garcia-Perez, M., Effect of cellulose crystallinity on the formation of a liquid intermediate and on product distribution during pyrolysis. Journal of Analytical and Applied Pyrolysis, 2013. 100: p. 56-66. 


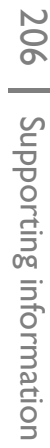




\section{H. Tables and figures (Chapter 6)}

Table H1. Product distribution of all the liquefaction experiments. Experiments performed with $90 \mathrm{wt} \%$ of solvent and $10 \mathrm{wt} \%$ of wood at $310^{\circ} \mathrm{C}$ for 30 minutes.

\begin{tabular}{|c|c|c|c|c|}
\hline Solvent & $\begin{array}{l}\text { Unconverted wood } \\
(\mathrm{C} \%)\end{array}$ & $\begin{array}{l}\text { Char } \\
(\mathrm{C} \%)\end{array}$ & $\begin{array}{l}\text { Gas } \\
(\mathrm{C} \%)\end{array}$ & $\begin{array}{l}\text { Bio-crude } \\
(\mathrm{C} \%)\end{array}$ \\
\hline 2,6-Diethylnaphthalene & 0.0 & 30.9 & 7.4 & 61.7 \\
\hline 1-Methylnaphthalene & 0.0 & 14.0 & 6.4 & 79.6 \\
\hline Phenanthrene & 0.0 & 20.3 & 12.5 & 67.2 \\
\hline Naphthalene & 0.0 & 21.3 & 8.9 & 69.8 \\
\hline Toluene & 0.0 & 28.7 & 4.3 & 67.0 \\
\hline Tetralin & 0.0 & 19.6 & 5.9 & 74.5 \\
\hline Decalin & 0.0 & 41.6 & 6.7 & 51.7 \\
\hline n-Undecane & 0.0 & 57.0 & 3.0 & 40.0 \\
\hline 1-Methoxynaphthalene & 0.0 & 9.9 & 6.7 & 83.4 \\
\hline 1,2-Dimethoxybenzene & 0.0 & 15.1 & 6.8 & 78.1 \\
\hline Anisole & 0.0 & 23.0 & 4.8 & 72.2 \\
\hline Guaiacol & 8.3 & 0.0 & 6.3 & 85.4 \\
\hline Phenol & 15.5 & 0.0 & 3.1 & 81.4 \\
\hline Catechol & 0.0 & 0.0 & 6.0 & 94.0 \\
\hline 1-Naphthol & 15.7 & 0.0 & 4.0 & 80.3 \\
\hline Pyrogallol & 0.0 & 11.7 & 31.1 & 57.2 \\
\hline 1-hexanol & 35.7 & 0.0 & 2.9 & 61.4 \\
\hline Hexanoic Acid & 0.0 & 42.0 & 2.5 & 55.5 \\
\hline Water (HTL) & 0.0 & 25.4 & 6.4 & 68.2 \\
\hline $\mathrm{N}_{2}$ & 0.0 & 79.1 & 7.0 & 13.9 \\
\hline CGO & 0.0 & 2.3 & 6.8 & 90.9 \\
\hline $\mathrm{LCO}$ & 0.0 & 26.9 & 11.3 & 61.8 \\
\hline VGO & 0.0 & 46.8 & 26.5 & 26.7 \\
\hline Hydrowax & 0.0 & 59.8 & 3.2 & 37.0 \\
\hline Guaiacol + water $(1: 1)$ & 0.0 & 0.0 & 1.7 & 98.3 \\
\hline Guaiacol + water $(8: 1)$ & 1.9 & 0.0 & 5.6 & 92.5 \\
\hline Guaiacol + water $(85: 5)$ & 8.7 & 0.0 & 5.2 & 86.1 \\
\hline Guaiacol + 1-methylnapthalene (1:1) & 3,1 & 0.0 & 6.5 & 90.4 \\
\hline 1-Methylnaphthalene + water $(8: 1)$ & 0,0 & 5.2 & 7.1 & 87.7 \\
\hline 1-naphthol + 1-methylnaphthalene (1:2) & 9,8 & 0.0 & 4.5 & 85.7 \\
\hline
\end{tabular}




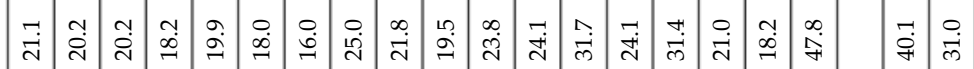

ने में

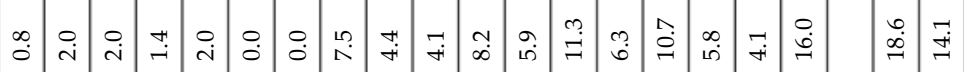

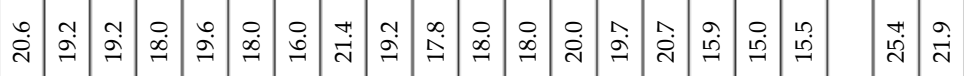

$$
\begin{aligned}
& \stackrel{+}{\vec{f}}
\end{aligned}
$$

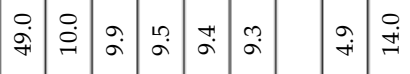

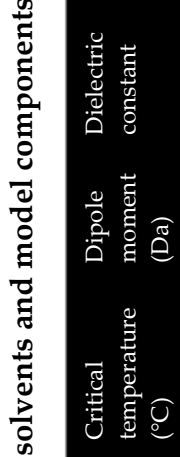

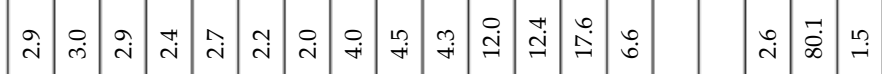

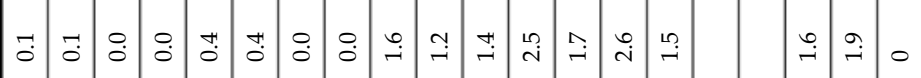

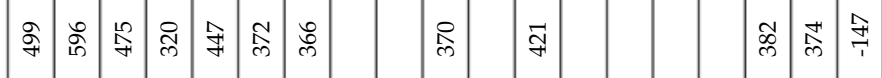

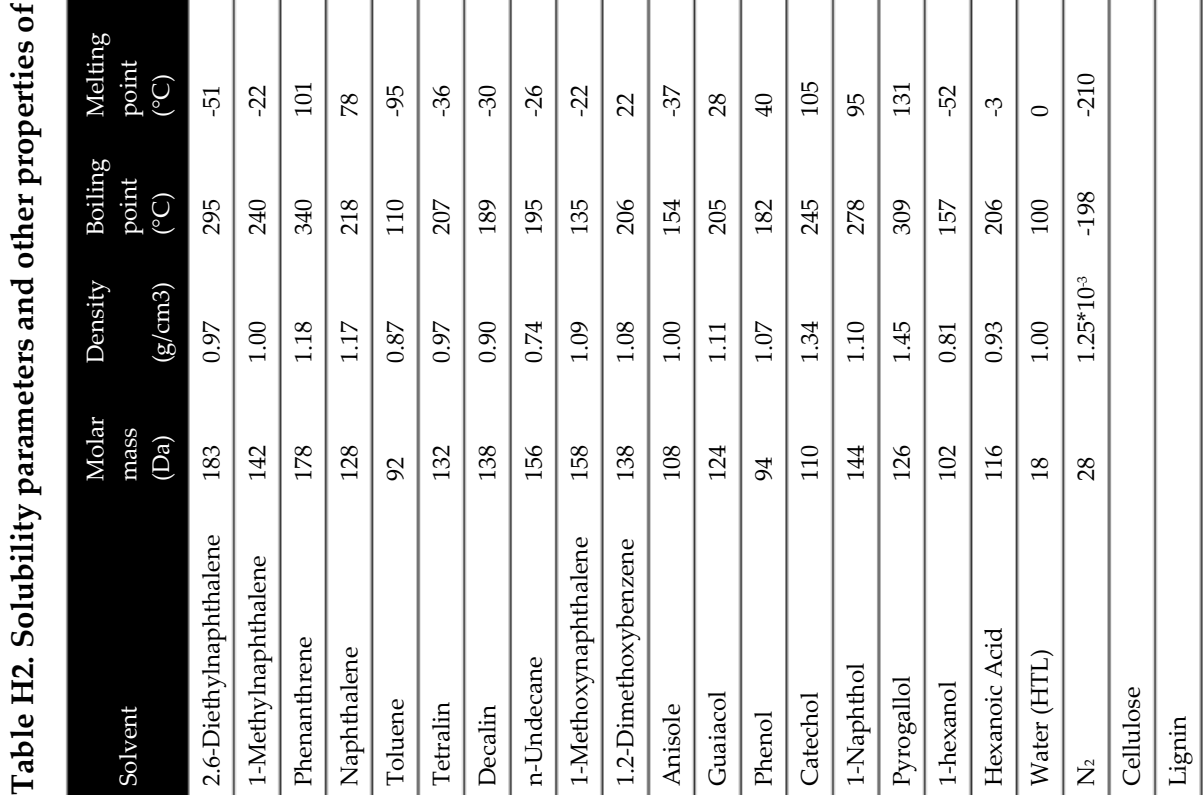


Table H3. Product distribution of the blank experiments for pyrogallol and CGO. Experiments performed with $100 \mathrm{wt} \%$ of solvent for $30 \mathrm{~min}$ at $310^{\circ} \mathrm{C}$.

\begin{tabular}{llll}
\hline Experiment & Solid yield $(\mathrm{C} \%)$ & Gas yield $(\mathrm{C} \%)$ & Bio-crude yield $(\mathrm{C} \%)$ \\
\hline Pyrogallol blank & 4.2 & 0.8 & 95.0 \\
\hline CGO blank & 0 & 0 & 100 \\
\hline
\end{tabular}
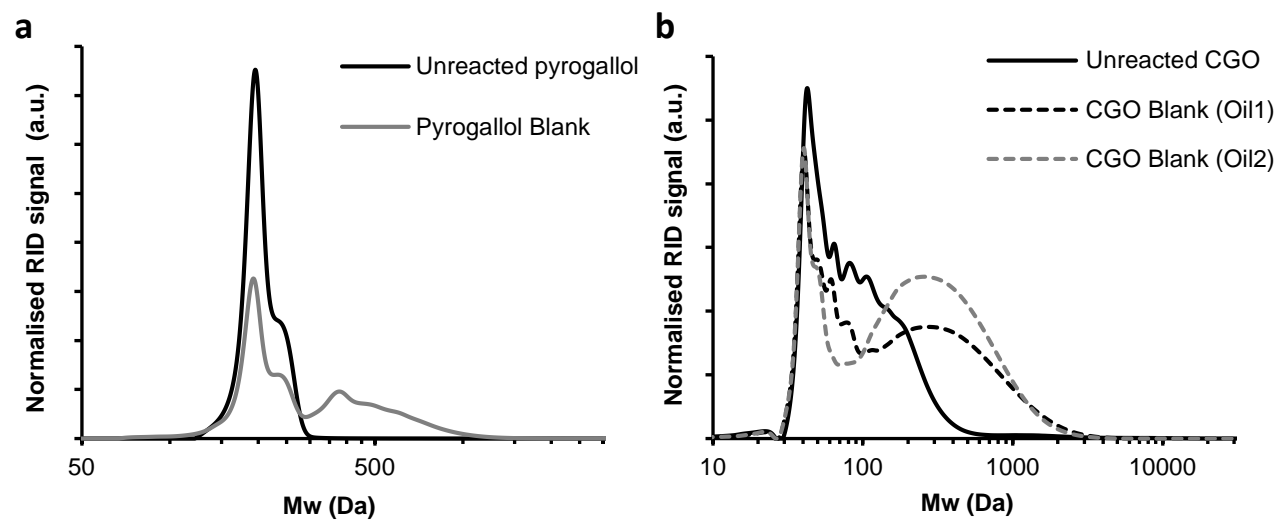

Figure H1. GPC chromatograms of pyrogallol (a) and CGO (b) unreacted and after being treated at $300^{\circ} \mathrm{C}$ for $30 \mathrm{~min}$. Phase 1 and 2 of CGO refer to the two liquid phases obtained after the experiment. 

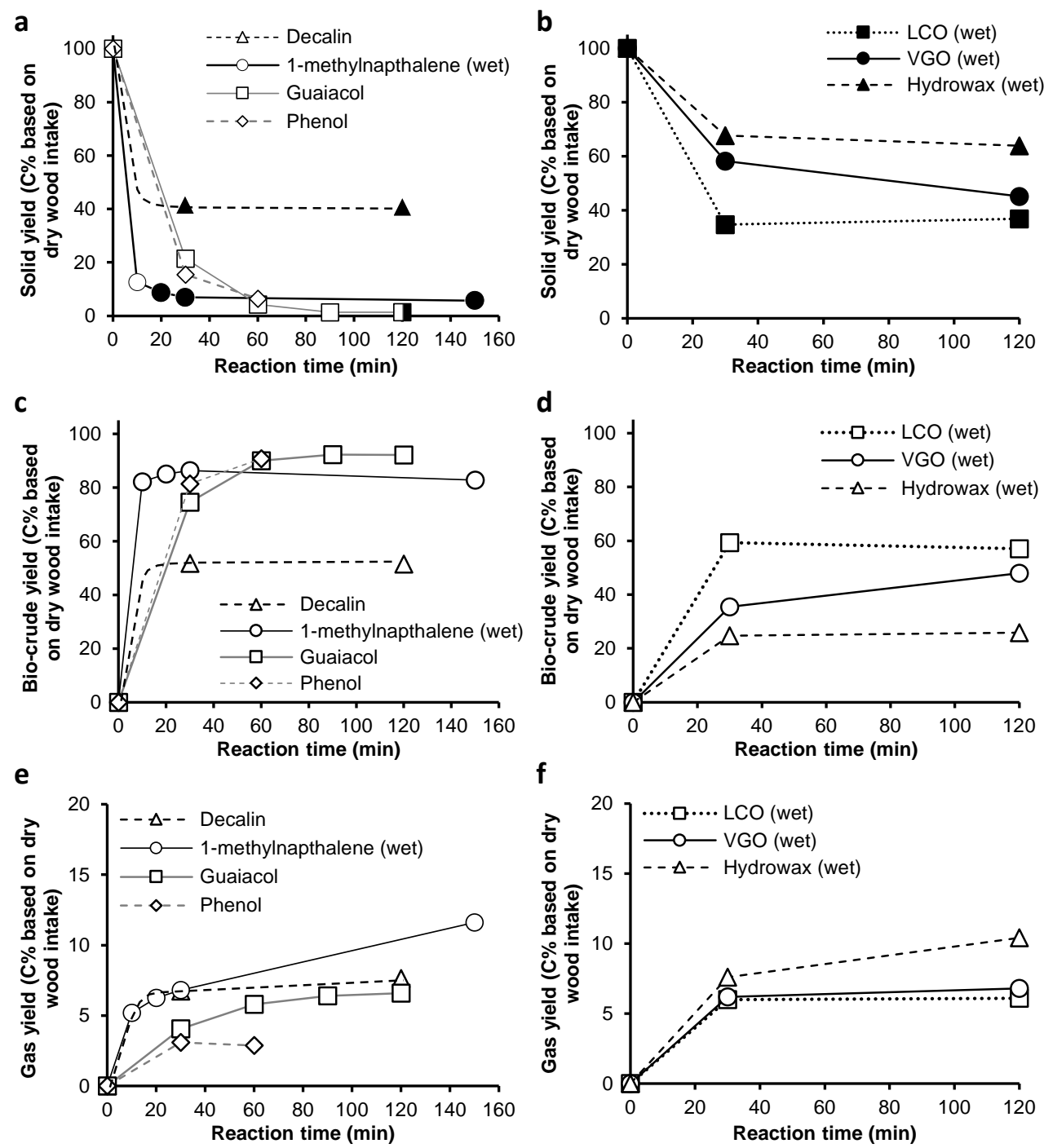

Figure H2. Product yields obtained with various liquefaction media at several reaction times. Guaiacol [1], decalin and phenol experiments performed with $90 \mathrm{wt} \%$ of solvent and $10 \mathrm{wt} \%$ of wood at $310^{\circ} \mathrm{C}$. 1-methylnaphthalene experiments performed with $85 \mathrm{wt} \%$ of 1methylnaphthalene, $5 \mathrm{wt} \%$ of water and $10 \mathrm{wt} \%$ of wood at $310^{\circ} \mathrm{C}$. Experiments with refinery streams (LCO, VGO and hydrowax) performed with $70 \mathrm{wt} \%$ of refinery stream, 10 $w t \%$ of water and $20 \mathrm{wt} \%$ of wood at $320^{\circ} \mathrm{C}$ [2]. In the graphs for solid yields, white symbols correspond to cellulose-like solid and blank symbols to char-like solid. The symbol with both white and black colours contained both types of solid residue. 


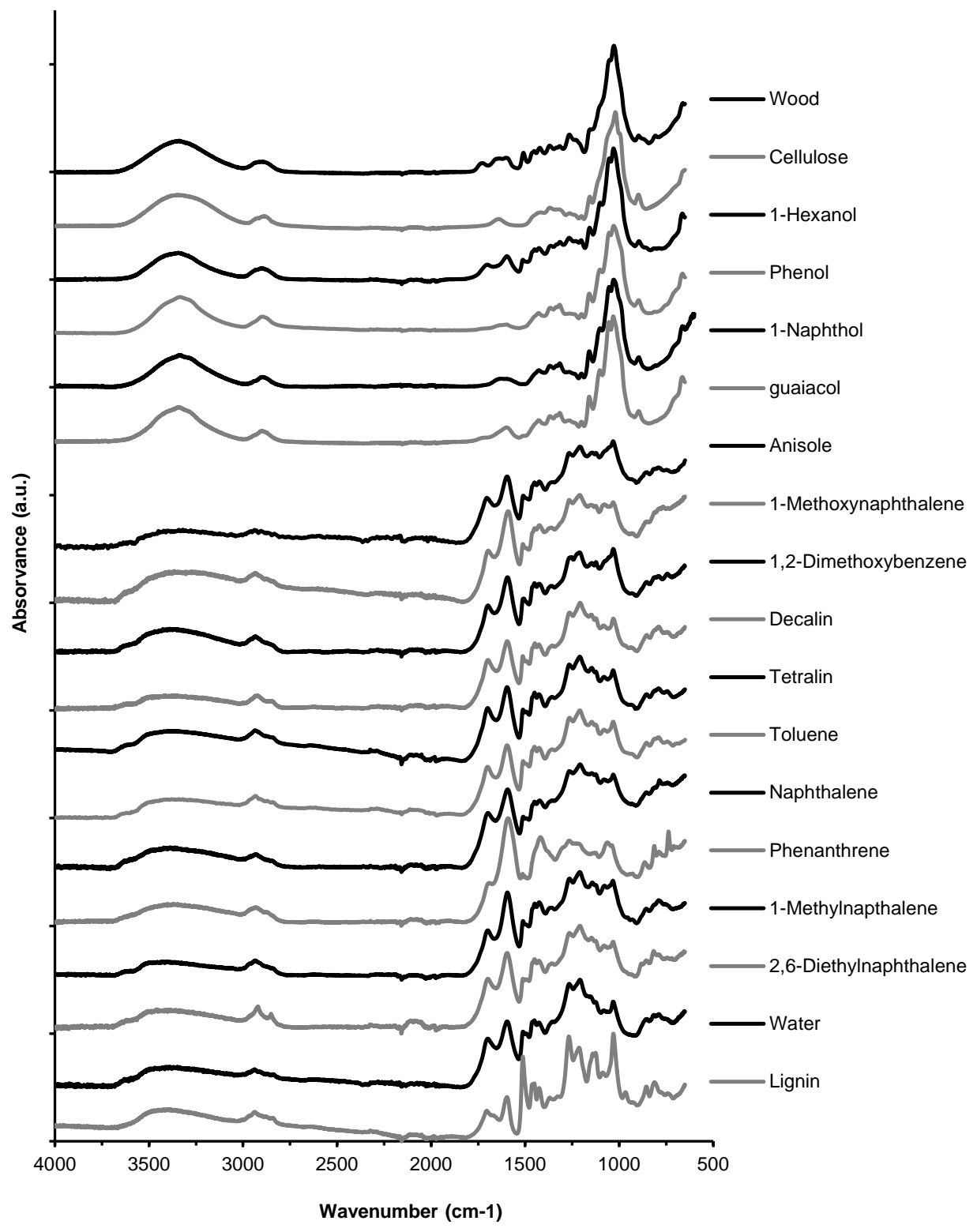

Figure H3. FTIR of the solid residues obtained after wood liquefaction in several organic solvents. 


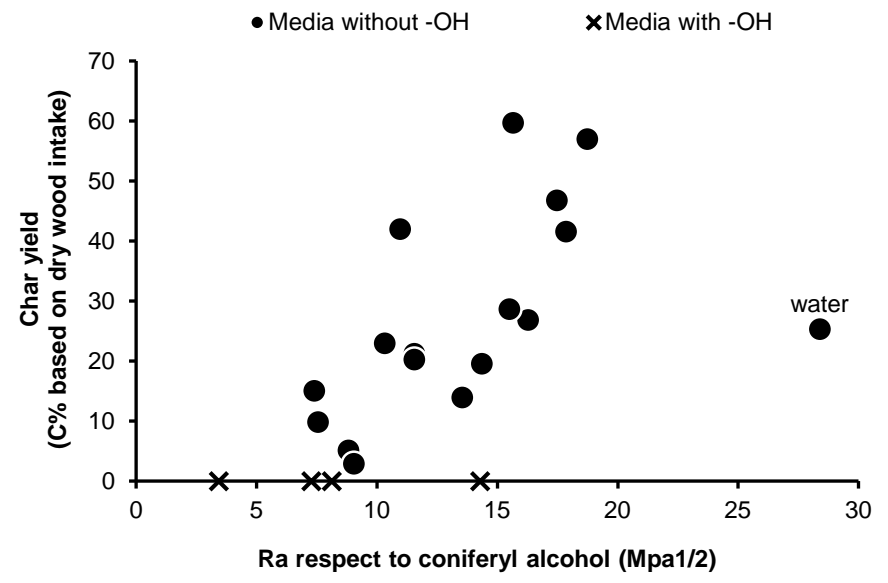

Figure H4. Correlation between solid yield and Ra of the liquefaction media respect to coniferyl alcohol. Only experiments with wood conversion $>95 \mathrm{C} \%$ are represented.

Table H4. Regression analysis of Solid yield correlation with Ra and Mw.

\begin{tabular}{|c|c|c|c|c|c|c|}
\hline \multicolumn{7}{|c|}{ SUMMARY OUTPUT } \\
\hline Multiple R & 0.95 & & & & & \\
\hline R square & 0.90 & & & & & \\
\hline Adjusted R square & 0.87 & & & & & \\
\hline Standard Error & 6.0 & & & & & \\
\hline Observations & 10 & & & & & \\
\hline \multicolumn{7}{|l|}{ ANOVA } \\
\hline & $S f$ & SS & MS & $F$ & Significance $F$ & \\
\hline Regression & 2 & 2196.6 & 1098.3 & 30.6 & 0.0004 & \\
\hline Residual & 7 & 250.9 & 35.9 & & & \\
\hline \multirow[t]{2}{*}{ Total } & 9 & 2447.5 & & & & \\
\hline & Coefficients & Std error & $t$ Stat & P-values & Lower $95 \%$ & Upper $95 \%$ \\
\hline Intercept & -100.13 & 21 & -4.7 & 0.002 & -150.96 & -49.40 \\
\hline Mw solvent & 0.05 & 0.01 & 3.9 & 0.006 & 0.02 & 0.09 \\
\hline Ra-cellulose & 3.87 & 0.7 & 5.6 & 0.0008 & 2.24 & 5.50 \\
\hline
\end{tabular}




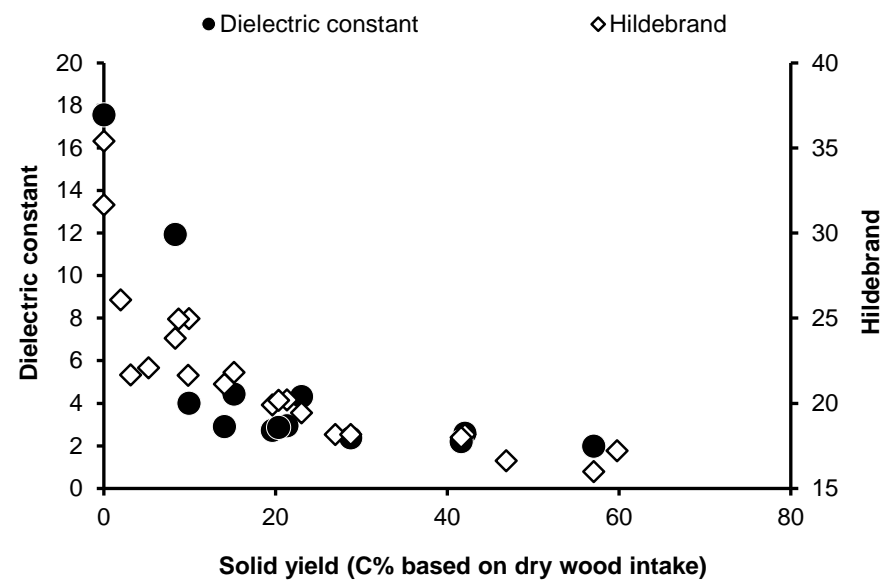

Figure H5. Correlations of bio-crude yield vs. Hildebrand and dielectric constant of the liquefaction solvents. 


\section{H.I. References}

1. van Rossum, G., Zhao, W., Castellvi Barnes, M., Lange, J.-P., and Kersten, S.R.A., Liquefaction of Lignocellulosic Biomass: Solvent, Process Parameter, and Recycle Oil Screening. ChemSusChem, 2013. 7(1): p. $253-259$.

2. Kumar, S., Direct Liquefaction of Lignocellulose: Exploration, Design and Evaluation of Conceptual Processes, in Sustainable Process Technology. 2015, University of Twente: Netherlands. p. 166. 


\section{Acknowledgements}

The first time I thought of coming to The Netherlands I was not sure if it was a good idea and if I was brave enough to live in a foreign country for half a year. After almost 6 years spent in Enschede, I can say without a doubt that this is one of the best decisions I made in my life. However, if something made this experience so valuable, it's not the great weather, the amazing food and the perfect sanity system in this country; but all the people that I met and that made me feel home.

Sascha, I would like to start thanking you for giving me the opportunity of staying in SPT to do my PhD. Thanks also for your supervision. Even though we only had few meetings, they were always useful and interesting. Thanks also for your efforts correcting my papers, even though they were full of complicated chemistry and analyses, and thanks for forcing me a bit into learning chemical engineering (even though I complained). Laura and Wim, I would also like to thank you for making my internship in SPT (TCCB at that time) possible. I would not be here today if you had not given me the opportunity of joining the group so many years ago. Thanks Jean-Paul for all your help during these years. I will always be amazed of how much you know about so many things. I learned a lot from you, and our long (but profitable) meetings have always been an incredible source of new ideas (and a lot of work). Guus, you were my supervisor at the beginning of my $\mathrm{PhD}$, and have also been a friend during these 6 years I have spent in The Netherlands. Unfortunately, you decided to abandon us, first by moving to Canada, and then to Shell Amsterdam ;). Even though we didn't have the chance to spend much time together in the last years, I will never forget some of our best moments, including the salsa dances or how you forced me to eat a grasshopper. I hope we can meet soon in Amsterdam together with Rebeca, Mark and Laia.

I would also like to thank the rest of the committee members. It is an honour that you accepted to be a part of my defence committee and I hope that we have a nice and fruitful discussion on the $27^{\text {th }}$ of May. 
During this PhD I had an amazing project partner. Shushil, thanks for always being ready to help and for letting me go first in all the presentations we did in Shell, knowing that I got less stressed if we did it that way. Thanks also for all the chicken curries and all the other Indian foods you cook for the group (or for yourself, I also ate from those more than once). Marco, Jan and Roger, I had the pleasure to supervise you during you master and bachelor projects. Thanks for all the nice work you did and for the good moments we shared together. I would also like to express my gratitude to those that helped me revising and finishing the thesis: Lisette (thanks so much for the corrections and the Dutch summary, my desktop is all yours, you've earned it!), Ehsan, Sandra, Özge and my paranymph Rens.

I would also like to thank all the technicians without whom I could not have done my $\mathrm{PhD}$. Erna, thanks for showing me all the analysis techniques we have in the lab and for helping me every time there were problems with the (stupid) GPC and with the (horrible) Micro-GCs. I think we did a very nice job arranging the GPC preparative fractionation system $\odot$. Thanks also to Benno, Johan and Karst. I was lucky that the $45 \mathrm{ml}$ autoclave was already built when I started the $\mathrm{PhD}$, but I could not have done all the experiments without your help every time I broke it. Thanks for repairing it the 100 times it had a leak, or the 10000000 times I broke the thermocouple (seriously, does it happen to everybody, or I have a special ability to destroy it?). Thanks for never complaining and fixing it so fast and with a smile on your faces. Thanks also for every time you helped me opening and closing the monstrous 5L autoclave and for not complaining (at least to me) that week I had to work in the HPL until 9 pm. I would also like to thank Bianka Snellink for her help with the NMR analyses. Yvonne, thank you so much for all the times I came to your office asking for help with administration problems, conference bills or just looking for new office material. You always welcomed me with a smile and helped me all you could. I knew that, no matter the problem I had, you would be able to solve it. I would also like to thank all the other members of the SPT team: Louis and Wim (or Dupond and Dupont), Boelo, Henk, Roel, Martin, Sam (my private babyitems provider ;) thanks sooo much!), Xiao Hua, Cindy (I like our cooking talks, and your food even more!), Ehsan (I'm happy that you came to live in the same building and that you are so lazy, I enjoyed your company every time we took the bus together!), Marek, Sandra, Varsha, Pushkar, Danielle, Stevia, Qian, Lisette, Özge, Thomas, Dion, Muthia. Thanks also to all ex- 
SPT members: Laura and Can (thanks for the days in Munich and for the help and inspiring talks about babies), Pavlina and Dimitris (parties are always more fun when you two are there!), Caroline, Michal, Maarten, Jose and Kate, Prasad (thanks for introducing me into Indian music, food and weddings), Lucia, Elia, Nancy, Caecilia, Diego, Maria, Ferran, Nick, ... You all have been my family in Enschede during these past 6 years, the people with whom I have spent most of my day, with whom I had fun, I shared a lot of coffees, teas and cakes, the ones who heard me complain when I was stressed, lost my bike (again) or when I could not sleep because of the matrass(es).

If there are some moments during the $\mathrm{PhD}$ that I will never forget are all the borrels, dinners and other parties that we shared together. I learned so many things from our borrels. First of all, there are two types of borrels, the normal borrels, and the crazy borrels. You can differentiate them taking into account various parameters: the amount of beer/wine available in the bar, the presence/absence of hard liquor and proper food (dinner), the amount of dancing involved, and the times we need to clean the bar before all stains and smell are gone. I learned that you can open a wine bottle using only a shoe, but I also learned that it isn't such a good idea when you are opening a bottle of Lambrusco. I learned that most Dutch people will dance only after consuming a minimum amount of beer and only with the appropriate (Dutch) music. I learned that it isn't a good idea to have a white board on your front door if there are crazy people parting in front of it. I learned that you don't need a lot of people to have a great party (Rens and Michal, I will never forget that borrel in which we went up and down the Meander building dancing with the music of Aqua and Going to Rio). I would specially like to express my gratitude to all my dancing colleagues, you all helped increasing the mood of the party: Jose (Mr la Bomba, it was always the best way to start the dancing time!), Pavlina, Laura, Sandra, Stevia, XiaoHua (how can you learn so fast?!), ...

What can I say about my two paranymphs, Rens and Stijn. We have shared so many things together! Office hours, borrels, dinners, parties, conferences, trips... It was great to spend time with you both in India and in Munich. Travelling it's always a thousand times better if you do it in good company! Stijn, thanks for all the dinners (I always like your Indonesian and Thai dishes) and for being my personal literature library; I don't know how many papers and 
authors I discovered thanks to you (it amazes me how much you know, even though you always say that Roel knows even more). Rens, thanks for teaching me new songs (although my favourite one will always be Go to Rio) and for making me realise I have such a horrible voice.

I would also like to thank all the members of the CPM group for all the moments we shared in borrels, BBQs and other activities: Leon (for the summer BBQs), Seshan (because meeting you and your wife on Saturday has already become a routine $(-)$ ), Bert, Karin and Tom (for granting me access to your labs and helping when needed); Shilpa and James (I love our dinner-game nights); Kamilla and Andrew (hope to share many more panda games); Kaisa (I had a great time in Pittsburgh, we have to go to a new escape room soon!); Joline and Arnout (great travelling company in China and game advisors); Masoud and Fatemeh (hope to see you again soon), Songbo, Cristina, Jose, Chau, Rolf, ...

Filipos, we met each other just few weeks after arriving to Enschede. All started by sharing some brunches and dinners together with Roger, Pepo and Julien. After that, Roger and I could not get rid of you anymore. Sometime later I also met Sherry (I am not sure yet who was more nervous the first time we all met at the carpet bar (-)). We have shared so many things after that day! It was amazing having you in Barcelona, bringing you to the best spots to eat hot chocolate, visiting the food market, seeing Filipos and Roger running like kids on the mountains... I will also never forget Sherry hanging in the tree-to-tree (sorry Sherry, but it was very funny), all the crepes on Sunday mornings, the Persian dinners and the game evenings. I have always felt we had two homes in Enschede (now in Arnhem). Damon and Saghar, thank you for so many amazing evenings and weekends! I hope we can share a lot more scape rooms, walks in Amsterdam, storytellings and late night movies (even though I am the only one that doesn't fall asleep). I also love our Persian dinners, and I will never forget Damon's cow tong (and his love for pressure cookers)! Next time, Roger and I have to cook you some Spanish food. Of course you will have to come to Enschede for that, because we know we are not allowed to enter to your kitchen ;). We also have to start arranging our trip to Japan (I'll be ready as soon as I can eat Shushi and the cherry trees flourish again $:)$ ). Pavel, thank you for all the shared moments: the tree-to-tree, the cinema sessions and the football matches (long long time ago). 
If there is something I enjoyed a lot during these last 3 years were the dancing lessons I had every Tuesday. Helia, you are an amazing dancer, and during your lessons I could always forget about the stress from work and just enjoy doing one of the things I love most. Thanks to you and all my dancing colleagues for those two amazing hours every week! Doris, thanks for giving me company on my way back home so many times, even when we had to walk during more than 20 minutes. I hope to enjoy many more evenings with you and Hilde, and hope that soon we can also have our Bollywood evening.

The hardest part of coming to The Netherlands was leaving my family back in Spain. After 6 years it is still difficult every time I miss a family reunion or a traditional festivity, but I know that, even in the distance, you are always there for me. It is amazing that, after so long, every time I come to Barcelona it feels as if I never left. Thanks to all of you for being how you are and for always showing how much you care about me. I love you all, and I couldn't be more proud of being a part of this family $(-)$.

I cannot finish these acknowledgements without thanking my partner in life. Roger, there are so many things I could thank you for... Thanks for making life so easy, so beautiful and so full of meaning. From the first moment we met at the University of Barcelona we both knew there was something special between us; after almost ten years I can say that I wouldn't change any of the moments I spent by your side. You are the one who made this (Dutch) adventure possible, you made me stronger, braver, more self-confident, and helped me all the times I felt homesick, sad or stressed. After so many years I can say that I've never loved you more than now, that I'm proud to call you my husband and that I can't wait to have our little Ayla with us.

Maria 


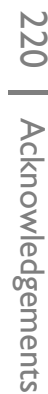


